\title{
Using Experimental Autoimmune Encephalomyelitis to Identify Prospective Treatments for Multiple Sclerosis
}

By

David O'Sullivan

A thesis submitted to the Victoria University of Wellington in fulfilment of the requirements for the degree of Doctor of Philosophy

Victoria University of Wellington 2012 


\section{Abstract}

Multiple sclerosis (MS) is an inflammatory disease, mediated by immune cells attacking the myelin sheaths that surround nerve axons. The autoimmune nature of this disease combined with heterogeneity in disease presentation and pathology makes MS a difficult disease to treat. Although some treatment options are currently available, there is strong demand for more effective treatment alternatives. Therefore, this thesis utilises an animal model of MS, experimental autoimmune encephalomyelitis (EAE), to identify new treatment strategies for MS. These studies encompass three separate lines of inquiry with the primarily focus on investigating the use and mechanisms of action by which microtubule-stabilising drugs (MSDs) modify EAE expression. Two other distinct and novel immunotherapies were also explored: the anti-psychotic drug, risperidone, and the Toll like receptor-9 and nucleotide oligomerization domain-containing protein 2 (TLR9/NOD2) agonist, MIS416.

MSDs are a class of anti-proliferative compounds, which can delay EAE disease onset and reduce disease burden. Administration of the MSD, paclitaxel, directly after EAE immunisation resulted in complete inhibition of antigen-specific encephalogenic responses within the spleen and attenuated responses within the lymph nodes. In contrast, administration of paclitaxel at a later time point did not alter antigen-specific responses, but inhibited immune cell infiltration into the central nervous system (CNS). Using in vivo proliferation and migration assays it was demonstrated that paclitaxel inhibited both immune cell proliferation and migration; indicating these two factors are likely to contribute to the disease modifying effects of MSDs. Futhermore, the combined administration of two MSDs, peloruside A and ixabepilone, resulted in synergistic disease suppression in vivo while the combination of paclitaxel and a currently used MS therapeutic, glatiramer acetate, also exhibited synergistic EAE suppression.

Risperidone is an atypical antipsychotic used to treat schizophrenia, however there is evidence that risperidone can also modulate the immune system. The current study 
demonstrated that risperidone reduced EAE disease severity and induced an increase in splenic $\mathrm{CD}^{+} \mathrm{T}$ cells and antigen-specific IFN- $\gamma$ production. Additionally, as macrophages have a crucial role in EAE disease development, the effect of risperidone on macrophage activation was explored. In cultured macrophages risperidone induced a reduction in IL-12 production and CD40 expression while increasing IL-10 production. These findings suggest that regulation of macrophage activation may contribute to the reduction in EAE.

MIS416 is a novel microparticle that stimulates TLR9 and NOD2 receptors. A phase 2A trial is currently underway to evaluate the effects of MIS416 in progressive MS patients, yet the mechanisms by which MIS416 alters the immune system are not completely understood. The current study found that MIS416 effectively but transiently reduces EAE and that IFN- $\gamma$ is necessary for this disease reduction. These experiments demonstrate that EAE is an appropriate model to further explore the precise mechanisms of action of MIS416.

In summary, the work conducted in this thesis has identified the potential of three separate approaches to modify inflammatory disease processes in an animal model of MS. This research provides an initial foundation from which further research can be conducted, with the ultimate goal of developing new treatments for MS. 


\section{Acknowledgements}

No major achievement happens in isolation and I owe a great deal of gratitude to many people for the support, encouragement, and sacrifices they have made to assist me over the past three years.

Firstly, I would like to thank my supervisor Asst. Prof. Anne La Flamme. Her guidance and support has been invaluable. I could not have asked for a better supervisor. Also, thank you to my co-supervisor Prof. John Miller who always provides useful insight and support, and is exactly what a Professor should be.

Thank you to all the people in the LAF lab: Marie, Degee, Ryan, Maddie, Lisa, Danielle, Marcus, Jenni, Prunita, Jonnel, Sara, Sarrabeth, and Bhagyashree; and also thanks to John's lab group. Collectively these people have provided a rich and enjoyable work environment.

I would particularly like to thank Marie, Maddie, Sarrabeth and Sara for helping out during some of the more epic experiments. Also to Lisa and Jenni for providing some much need proofreading. Thank you to Ariane and Marcus for the all useful discussion and support.

Thanks to Dr. Peter Northcote and the Natural Products Group in the VUW Chemistry Department for providing peloruside A and laulimalide. Thanks to Dr. Jill Webster and Rebecca Girvan from innate immunotherapeutics, for providing MIS416 and for the serum cytokine analysis. Thanks to Douglas pharmaceuticals for providing risperidone.

Thanks to my parents for the baby sitting duties and all round support during the last few years. Thanks also to the rest of my family, and my son Hugo, for their encouragement.

Finally I would like to thank my wife. Kim has been incredibly tolerant and supportive, and has made many sacrifices to help me achieve my goals. 


\section{Table of Contents}

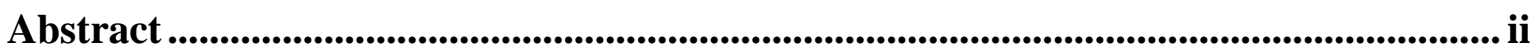

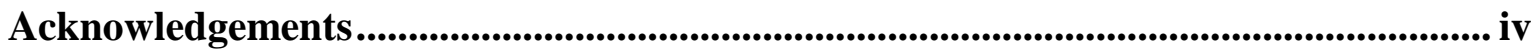

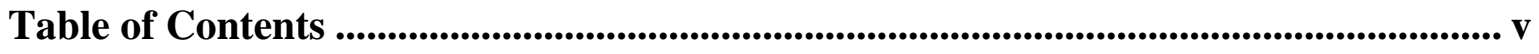

List of Figures ......................................................................................................................... ix

List of Tables............................................................................................................................. xiii

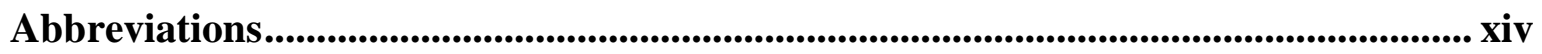

Chapter 1: General Introduction ........................................................................................... 1

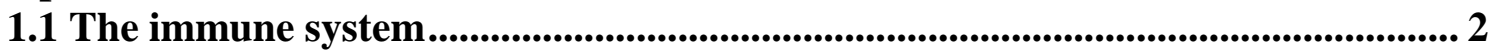

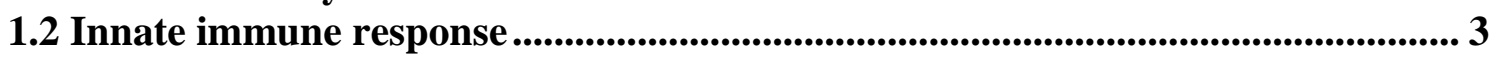

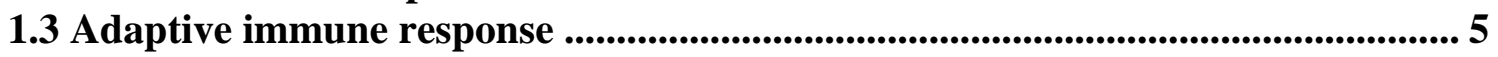

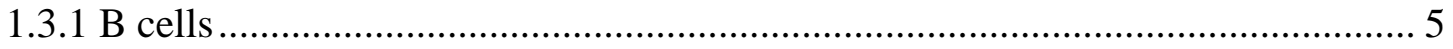

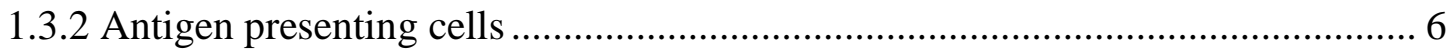

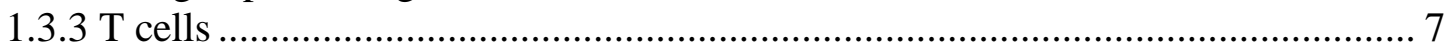

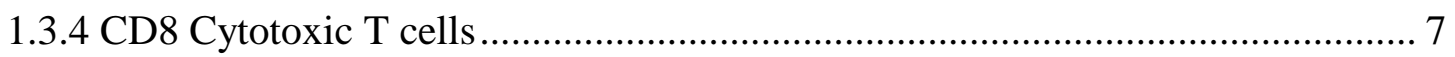

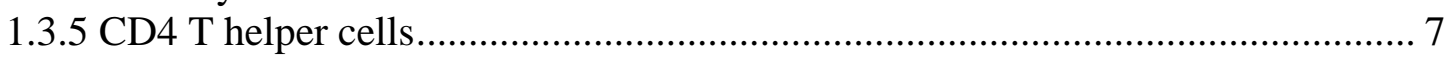

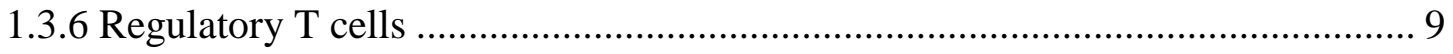

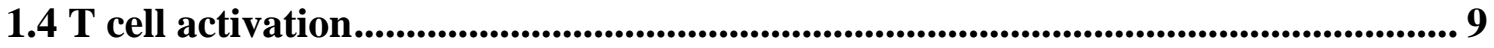

1.5 Immune tolerance.............................................................................................................. 10

1.6 Multiple sclerosis ........................................................................................................ 13

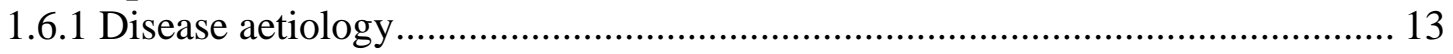

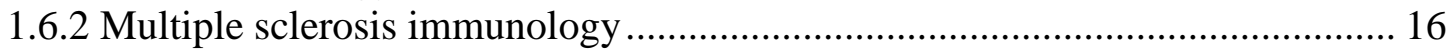

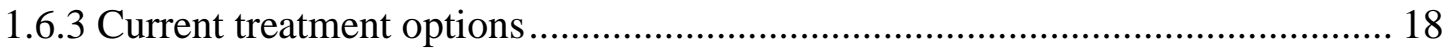

1.7 Animal models of multiple sclerosis ............................................................. 21

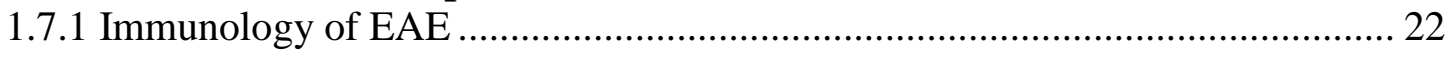

1.8 Overall aims and objectives ..................................................................................... 26

Chapter 2:_Materials and Methods.............................................................................................. 27

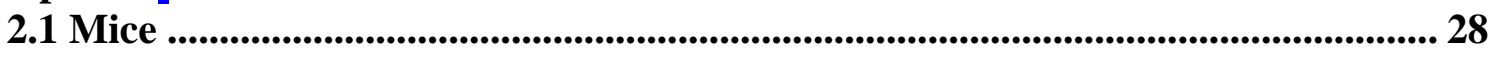

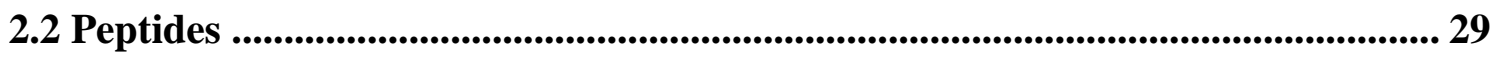

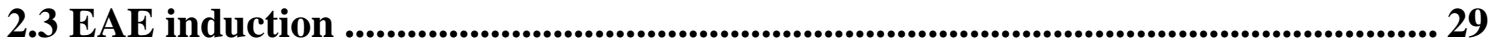

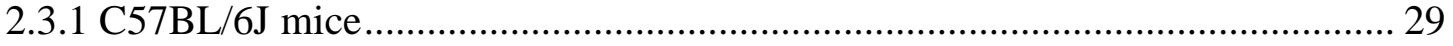

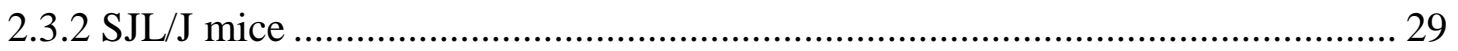

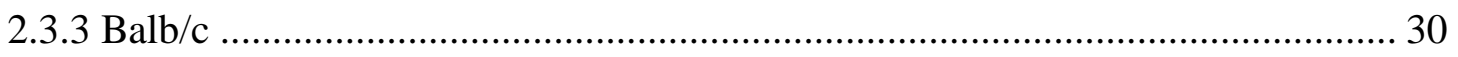

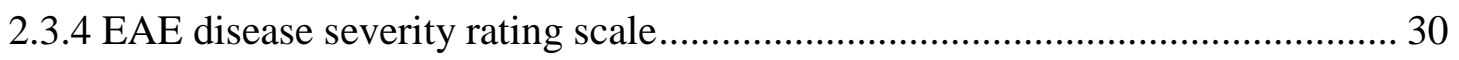

2.4 Compounds for in vivo use...................................................................................... 30

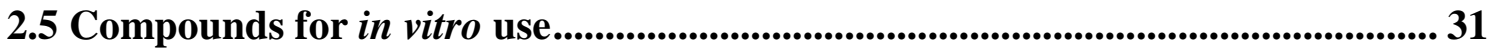

2.6 In vivo techniques ............................................................................................................... 31

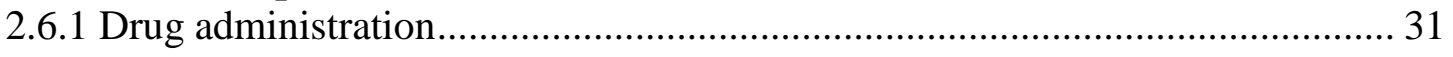

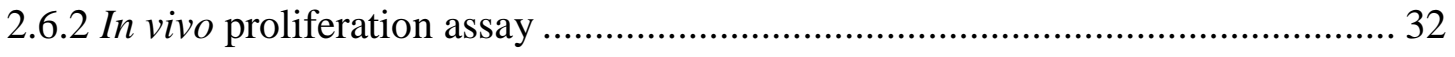

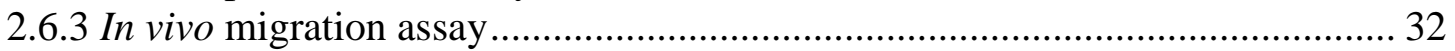

2.6.4 In vivo microtubule-stabilising drug combination treatments ......................... 33 


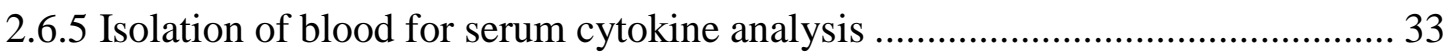

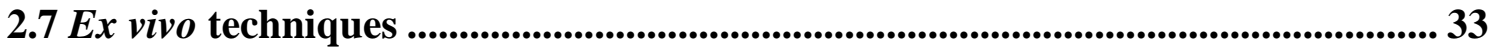

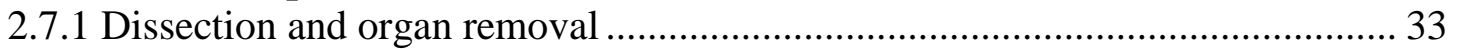

2.7.2 Preparation of a single cell suspension from lymphoid organs ........................ 34

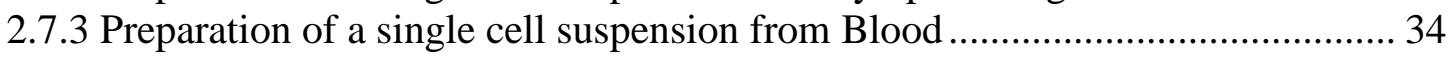

2.7.4 Preparation of a single cell suspension from the spinal cord ........................... 34

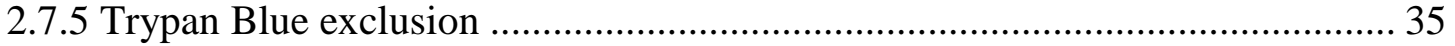

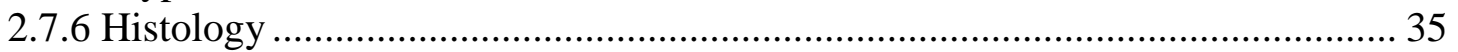

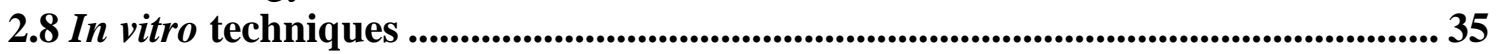

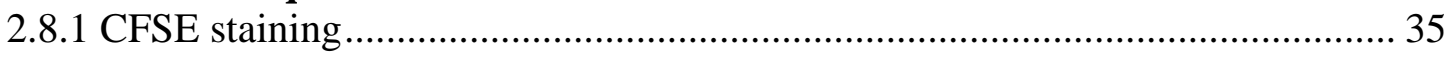

2.8.2 Preparation of bone marrow derived macrophages ...................................... 35

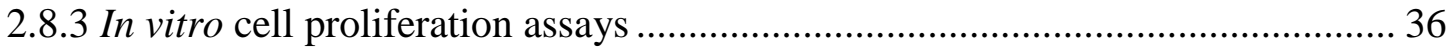

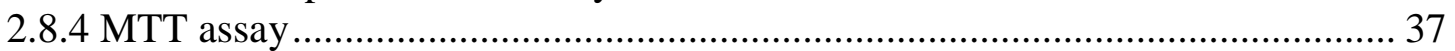

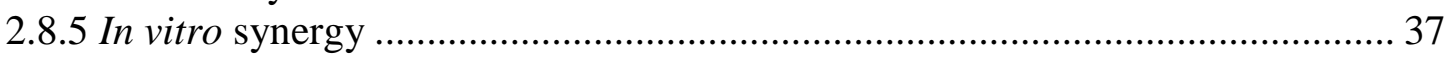

2.9 Cytokine multiplex assays ............................................................................ 38

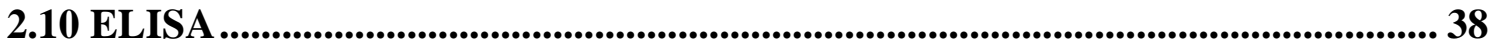

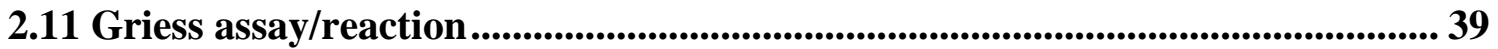

2.12 General antibody staining and preparation of cells for flow cytometry ........... 39

2.13 Graphs and Statistics .................................................................................... 40

Chapter 3:-Using microtubule-stabilising drugs to treat experimental autoimmune

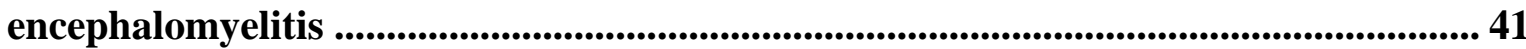

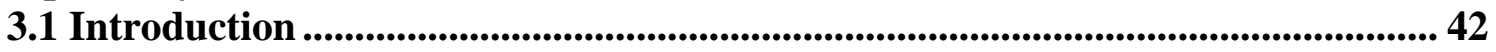

3.2 Aims and Objectives.............................................................................................. 44

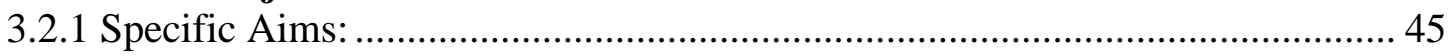

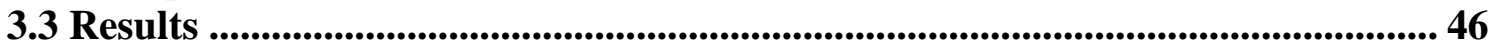

3.3.1 Paclitaxel and docetaxel have similar disease modifying effects and toxicity .. 46

3.3.2 Paclitaxel and docetaxel treatment does not inhibit the development of antigen-

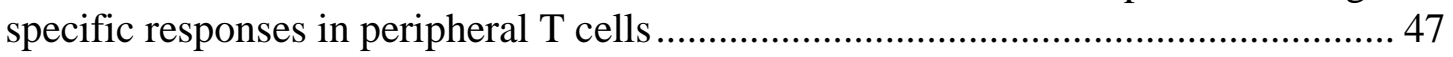

3.3.3 Treating after the initial inflammatory response delays disease onset .............. 49

3.3.4 A dual dosing regime delays disease but does not reduce long-term disease

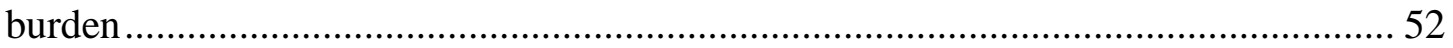

3.3.5 Ixbepilone alters disease expression without causing toxicity .......................... 55

3.3.6 Treating with ixabepilone after disease onset ............................................. 58

3.3.7 Paclitaxel and ixabepilone reduce disease expression in a Balb/c EAE model . 60

3.3.8 Paclitaxel inhibits disease expression in a relapsing-remitting EAE model ...... 61

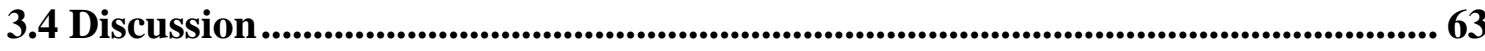

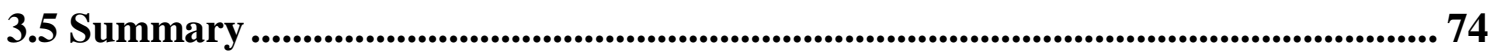

Chapter 4:_Identifying microtubule-stabilising drugs mechanisms of action ............. 75

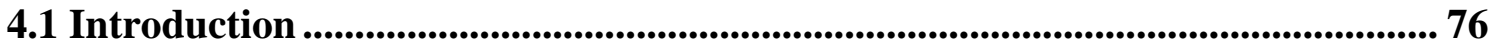

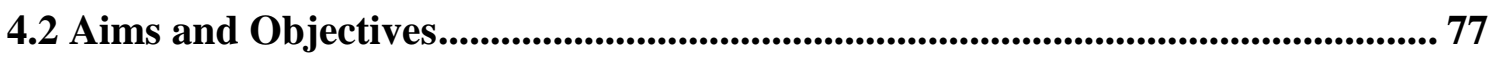

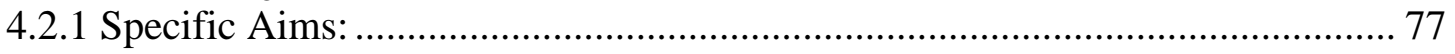

4.3 Results ............................................................................................................ 78

4.3.1 Spleen and LN cellularity is altered following paclitaxel treatment immediately

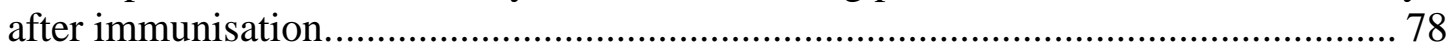


4.3.2 Antigen-specific responses are impaired in spleen and LN cells following paclitaxel treatment immediately after immunisation ............................................ 79

4.3.3 Immune cell infiltration into the spinal cord is inhibited following d0-4 or d6-12

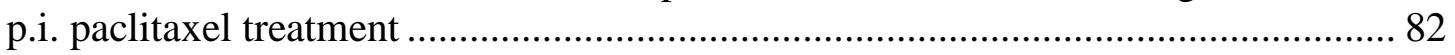

4.3.4 Administration of paclitaxel on d0-4p.i. has a different effect on spleen and dLN

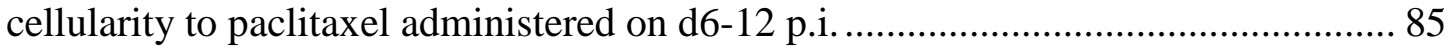

4.3.5 Antigen-specific responses in spleen and LN cells are unaffected following d6-

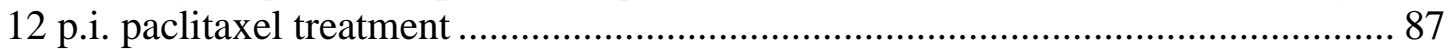

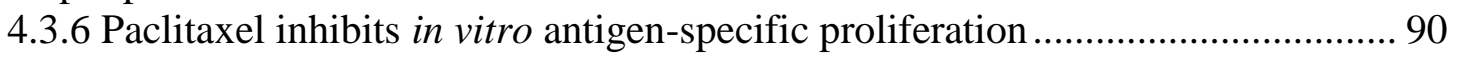

4.3.7 Paclitaxel inhibits in vivo antigen-specific proliferation ................................ 93

4.3.8 Paclitaxel inhibits in vivo immune cell migration ............................................ 97

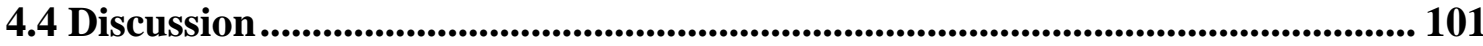

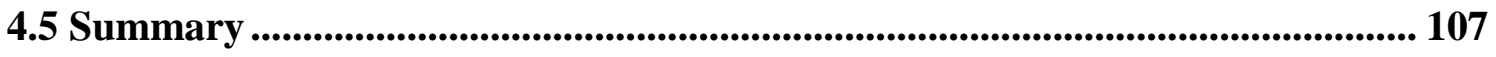

Chapter 5:_Identifying the synergistic potential of microtubule-stabilising drugs ... 108

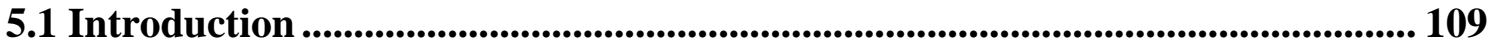

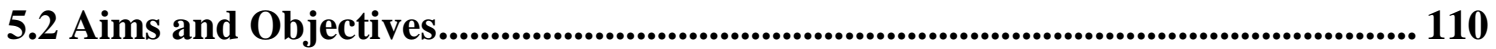

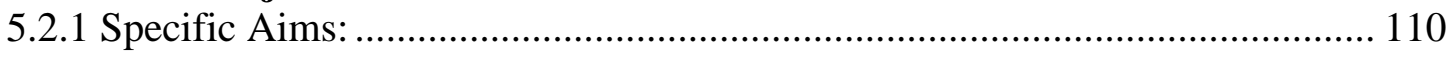

5.3 Results ............................................................................................................... 111

5.3.1 Identifying the synergic potential of MSDs to inhibit in vitro proliferation.... 111

5.3.2 Peloruside A and ixabepilone act synergistically to inhibit EAE ................... 115

5.3.3 Paclitaxel pre-treatment improves the efficacy of glatiramer acetate in the

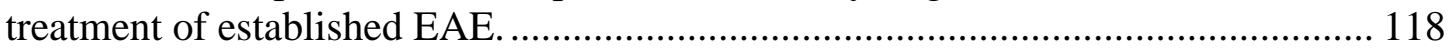

5.4 Discussion ................................................................................................................... 120

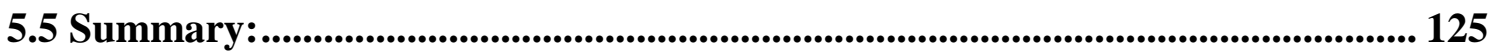

Chapter 6: Using risperidone to treat experimental autoimmune encephalomyelitis

6.1 Introduction

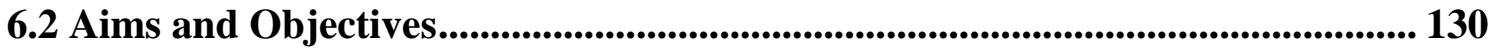

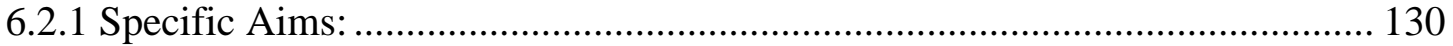

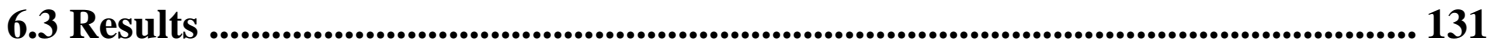

6.3.1 Risperidone alters macrophage activation............................................... 131

6.3.2 Treatment of mice with risperidone reduces disease severity of EAE............. 134

6.3.3 Risperidone treatment alters $\mathrm{T}$ cell populations in the spleen ......................... 136

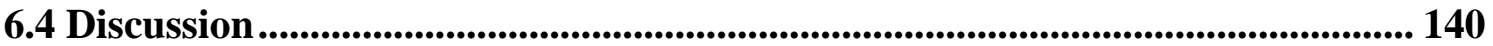

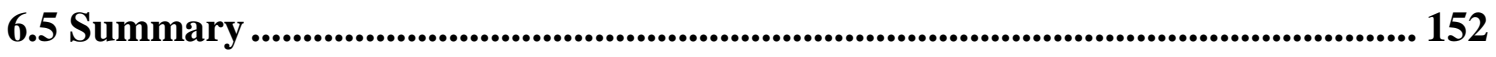

Chapter 7:_Using MIS416 to treat experimental autoimmune encephalomyelitis .... 153

7.1 Introduction ..................................................................................................................... 154

7.2 Aims and Objectives........................................................................................ 156

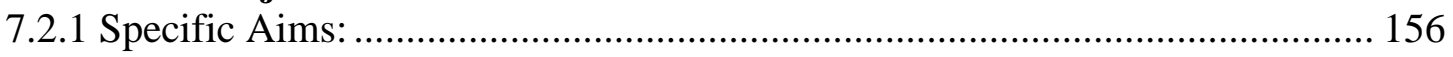

7.3 Results .......................................................................................................... 157

7.3.1 Administration of MIS416 on the day of immunisation reduces EAE disease

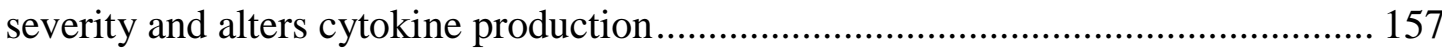

7.3.2 Administration of MIS416 at disease onset reduces EAE disease severity ..... 158 
7.3.3 Weekly administration of MIS416 reduces EAE disease severity and alters

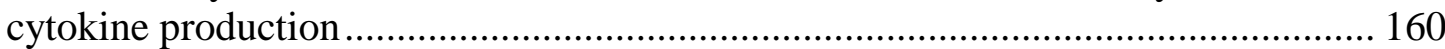

7.3.4 IFN- $\gamma$ signalling is necessary for MIS416 induced disease modification ........ 162

7.5 Summary .................................................................................................................................. 171

Chapter 8: General Discussion...................................................................................... 172

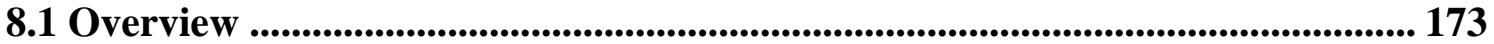

8.2 Microtubule-stabilising drugs ..................................................................... 174

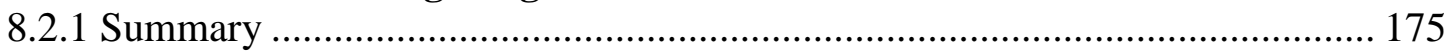

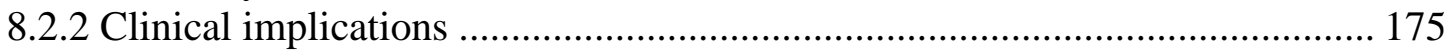

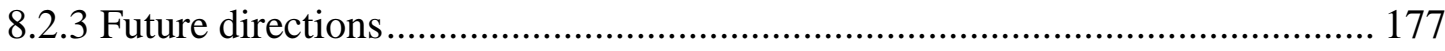

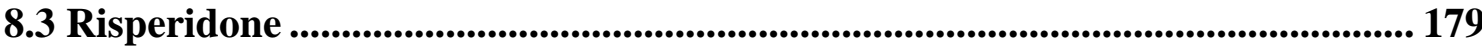

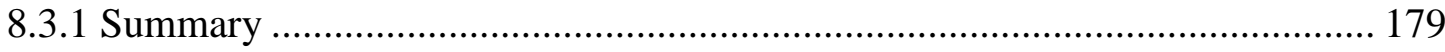

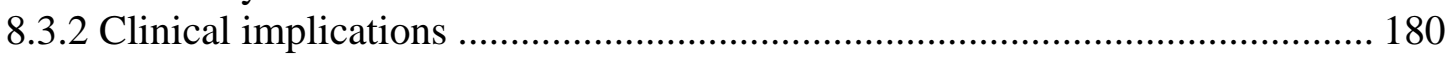

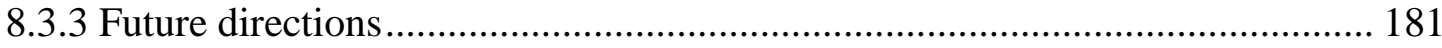

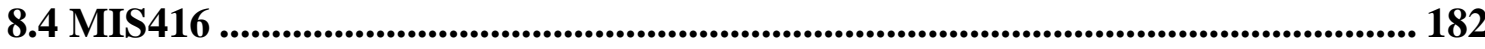

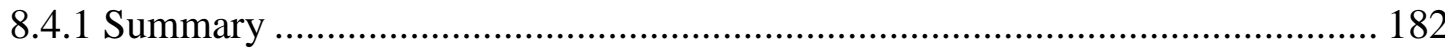

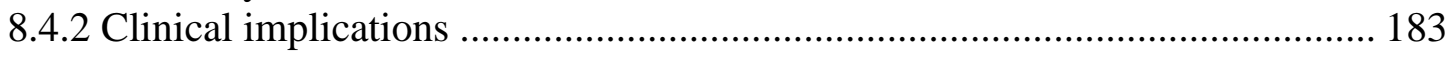

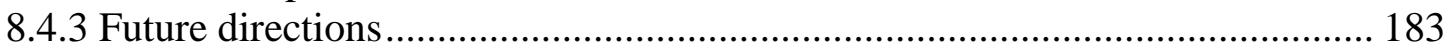

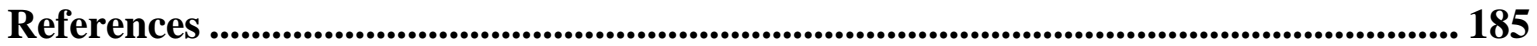

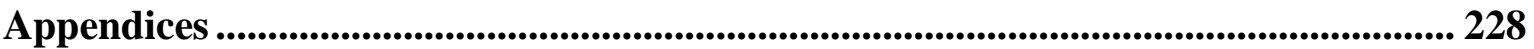

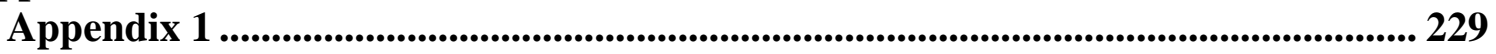

Appendix 2 ............................................................................................................. 230

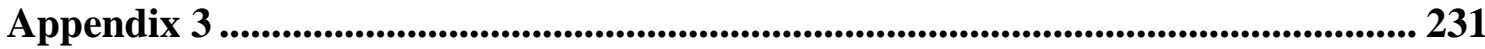

Appendix 4 .................................................................................................................................... 232 


\section{List of Figures}

\section{Chapter 3}

Figure 3.1

Paclitaxel or docetaxel treatment (d0-4 p.i.) delays EAE disease onset

Figure 3.2

Paclitaxel or docetaxel treatment (d0-4 p.i.) does not inhibit ex vivo antigen-specific proliferation

Figure 3.3

Paclitaxel or docetaxel treatment (d0-4 p.i.) does not alter spleen cellularity

Figure 3.4

Paclitaxel or docetaxel treatment (d0-4 p.i.) does not inhibit ex vivo IFN- $\gamma$

production.

Figure 3.5

Paclitaxel or docetaxel treatment (d6-10 p.i.) delays EAE disease onset.

Figure 3.6

Paclitaxel or docetaxel treatment (d6-10 p.i.) does not chronically alter spleen or lymph node cellularity

Figure 3.7

Paclitaxel or docetaxel treatment (d6-10 p.i.) does not inhibit ex vivo antigen-specific

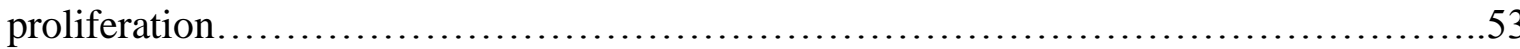

Figure 3.8

Paclitaxel or docetaxel treatment (d6-10 p.i.) does not inhibit ex vivo IFN- $\gamma$

production

Figure 3.9

A dual dosing regimen of paclitaxel or docetaxel does not improve in vivo efficacy....

Figure 3.10

Ixabepilone treatment (d6-12 p.i.) delays EAE onset and severity

Figure 3.11

Ixabepilone treatment (d6-12 p.i.) exhibits similar efficacy to paclitaxel in delaying EAE

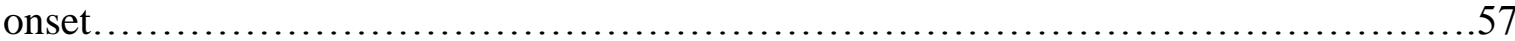

Figure 3.12

Ixabepilone treatment (d6-10 p.i.) does not chronically alter spleen or lymph node cellularity

Figure 3.13

Ixabepilone (d6-12 p.i.) does not inhibit ex vivo antigen-specific proliferation.

Figure 3.14

Ixabepilone reduces disease severity when administered after EAE onset

Figure 3.15

Paclitaxel or Ixabepilone inhibits the development of EAE in Balb/c mice

Figure 3.16

Paclitaxel reduces disease expression in a relapsing-remitting EAE model.... 
Figure 3.17 Paclitaxel, docetacel or ixabepilone treatment is more protective in less severe

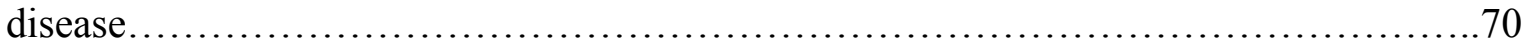

Figure 3.18

Paclitaxel, docetacel or ixabepilone treatment has a protective effect in EAE............70

\section{Chapter 4}

\section{Figure 4.1}

Spleen and lymph node cellularity is altered immediately after paclitaxel (d0-4p.i)

treatment

Figure 4.2

Ex vivo antigen-specific proliferation of spleen and lymph node cells is inhibited immediately after paclitaxel treatment (d0-4p.i).

Figure 4.3

Ex vivo nitric oxide production is not altered in spleen and lymph node cells immediately after paclitaxel treatment (d0-4p.i)...

Figure 4.4

Ex vivo cytokine production is not altered in spleen and lymph node cells immediately after paclitaxel treatment $(\mathrm{d} 0-4 \mathrm{p} . \mathrm{i})$.

Figure 4.5

Paclitaxel treated mice do not exhibit disease symptoms 13 days after immunisation

Figure 4.6

Paclitaxel inhibits immune cell infiltration into the CNS

Figure 4.7

Paclitaxel alters spleen cellularity when administered on d0-4 p.i. or on d6-12 p.i.

Figure 4.8

Paclitaxel does not inhibit ex vivo antigen-specific proliferation when administered on d6-12 p.i.

Figure 4.9

Antigen-specific nitric oxide production is not altered in ex vivo spleen and lymph node cells from paclitaxel treated mice.

Figure 4.10

Antigen-specific cytokine production is not altered in ex vivo spleen and lymph node cells from paclitaxel treated mice..................................................91

Figure 4.11

In vitro paclitaxel inhibits antigen-specific proliferation.

Figure 4.12

In vitro paclitaxel and docetaxel inhibit proliferation in splenocytes isolated from $\mathrm{EAE}$ immunised mice.

Figure 4.13

Paclitaxel attenuates in vivo antigen $\mathrm{T}$ cell proliferation within the lymph node.

Figure 4.14

Paclitaxel inhibits in vivo antigen $\mathrm{T}$ cell proliferation within the blood and spleen 
Figure 4.15

In vivo migration of $\mathrm{T}$ cells and macrophages is inhibited by paclitaxel.

101

Figure 4.16

In vivo migration of adoptively transferred antigen-specific cells is not significantly altered

following paclitaxel treatment.

\section{Chapter 5}

Figure 5.1

Determining the anti-proliferative activity of MSDs in splenocytes

Figure 5.2

Determining the dose response effects of peloruside A and ixabepilone in vivo

Figure 5.3

Peloruside and ixabepilone act synergistically to inhibit EAE

Figure 5.4

Weight change following in vivo administration of peloruside and ixabepilone

Figure 5.5

Peloruside and ixabepilone act synergistically to reduce EAE disease burden

Figure 5.6

Paclitaxel enhances the disease modifying effects of glatiramer acetate...

Figure 5.7

Paclitaxel and glatiramer acetate synergistically reduce disease burden...

\section{Chapter 6}

Figure 6.1

Risperidone alter macrophage viability only at high concentrations

Figure 6.2

Risperidone reduces IL-12 and increase IL-10 production in LPS stimulated macrophages

Figure 6.3

Risperidone reduces CD40 expression of LPS stimulated macrophages....

\section{Figure 6.4}

Risperidone reduces EAE disease severity

Figure 6.5

High doses of risperidone do not reduce EAE disease severity

Figure 6.6

Risperidone does not significantly reduce the size of spinal cord lesions

Figure 6.7

Risperidone increase the proportion of CD4 T cells within the spleen

Figure 6.8

Risperidone does not alter total cell numbers in the spleen or lymph node....

Figure 6.9

Risperidone does not alter ex vivo antigen-specific proliferation in spleen or lymph node cells.

Figure 6.10

IFN- $\gamma$ production is increased following antigen-specific stimulation of splenocytes from risperidone treated mice. 


\section{Figure 6.11}

IFN- $\gamma$ production is increased following polyclonal stimulation of lymph node cells from risperidone treated mice........................................................... 143

\section{Chapter 7}

Figure 7.1

MIS416 reduces EAE severity when administered on d0 p.i.

Figure 7.2

MIS416 increases serum IFN- $\gamma$ and IL-6 levels. ...

Figure 7.3

MIS416 reduces disease severity when administered on d12 p.i. but not d20 p.i.

Figure 7.4

MIS416 Reduces disease severity when administered weekly at a low dose

Figure 7.5

Weekly low dose MIS416 increases serum IFN- $\gamma$ during disease onset

Figure 7.6

Administration of anti-IFN- $\gamma$ antibody does not alter the disease course in MIS416 treated

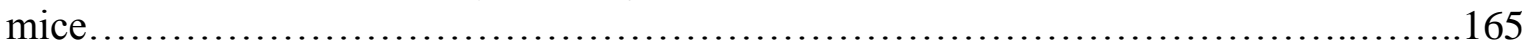

Figure 7.7

Administration of anti-IFN- $\gamma$ antibodies causes increased detection of IFN- $\gamma$ .165

Figure 7.8

MIS416 does not reduce disease severity in IFN- $\gamma$ deficient mice.

\section{Chapter 8}

Figure 8.1

How MSDs risperidone or MIS416 could potentially alter the EAE disease pathogenesis 


\section{List of Tables}

Chapter 3

Table 3.1 Paclitaxel and docetaxel alter EAE disease parameters.....................58

Table 3.2 Paclitaxel and Ixabepilone alter EAE disease parameters..................58

Chapter 5

Table 5.1

IC50 values of individual MSD compounds..........................111

Table 5.2 CI values for peloruside A and laulimalide drug combinations............114

Table 5.3 CI values for peloruside A and docetaxel drug combinations..............114

Table 5.4 CI values for peloruside A and ixabepilone drug combinations...........114

Table 5.5 CI values for laulimalide and ixabepilone drug combinations..............114 


\section{Abbreviations}

\begin{tabular}{|c|c|}
\hline $\mathrm{APC}$ & antigen presenting cell \\
\hline BrdU & 5-bromo-2-deoxyuridine \\
\hline cAMP & Cyclic adenosine monophosphate \\
\hline CCL5 & chemokine (C-C motif) ligand 5 \\
\hline CCR5 & chemokine ( $\mathrm{C}-\mathrm{C}$ motif) receptor 5 \\
\hline $\mathrm{CD}$ & cluster of differentiation \\
\hline CFA & complete Freund's adjuvant \\
\hline CFDA-SE & carboxyfluorescein diacetate $\mathrm{N}$-succinimidyl ester \\
\hline CFSE & carboxyfluorescein succinimidyl ester \\
\hline $\mathrm{CI}$ & combination index \\
\hline Con A & Concanavalin A \\
\hline $\mathrm{CPM}$ & Counts per minute \\
\hline CTCM & complete $\mathrm{T}$ cell media \\
\hline CTL & cytotoxic lymphocyte \\
\hline $\mathrm{d}$ & day \\
\hline $\mathrm{D}_{(1 \text { or } 2)}$ & Dopamine receptor ( 1 or 2$)$ \\
\hline $\mathrm{DC}$ & dendritic cell \\
\hline $\mathrm{dLN}$ & inguinal lymphnode that drains the EAE immunisation site \\
\hline DMSO & dimethyl sulfoxide \\
\hline dPBS & Dulbecco's phosphate buffered saline \\
\hline DTX & docetaxel \\
\hline EAE & eperimental autoimmune encephalomyelitis \\
\hline ELISA & enzyme-inked immunosorbant assay \\
\hline FACS & fluresent activatef cell sorting \\
\hline FCS & foetal calf serum \\
\hline FDA & Food and Drug Administration \\
\hline Foxp3 & forkhead box p3 \\
\hline $\mathrm{g}$ & gravity \\
\hline GA & glatiramer acetate \\
\hline GATA3 & GATA-binding protein 3 \\
\hline $\mathrm{Gi}$ & inhibitory regulative G-protein \\
\hline GM-CSF & granulocyte-macrophage colony-stimulating factor \\
\hline GPCR & G protein-coupled receptors \\
\hline $\mathrm{H}_{(1,2,3 \text { or } 4)}$ & Histamine receptors $1,2,3$ or 4 \\
\hline HLA & human leukocyte antigen \\
\hline HPLC & High-performance liquid chromatography \\
\hline HRP & horseradish peroxidase \\
\hline $\mathrm{IC}_{50}$ & inhibitory concentration 50 \\
\hline IFN- & interferon \\
\hline $\operatorname{IgG}$ & Immunoglobulin $\mathrm{G}$ \\
\hline IL- & interleukin \\
\hline $\mathrm{iPTX}_{\mathrm{d} 0-4}$ & immunised paclitaxel treated daily on $0-4$ p.i. $(20 \mathrm{mg} / \mathrm{kg} / \mathrm{d})$ \\
\hline
\end{tabular}




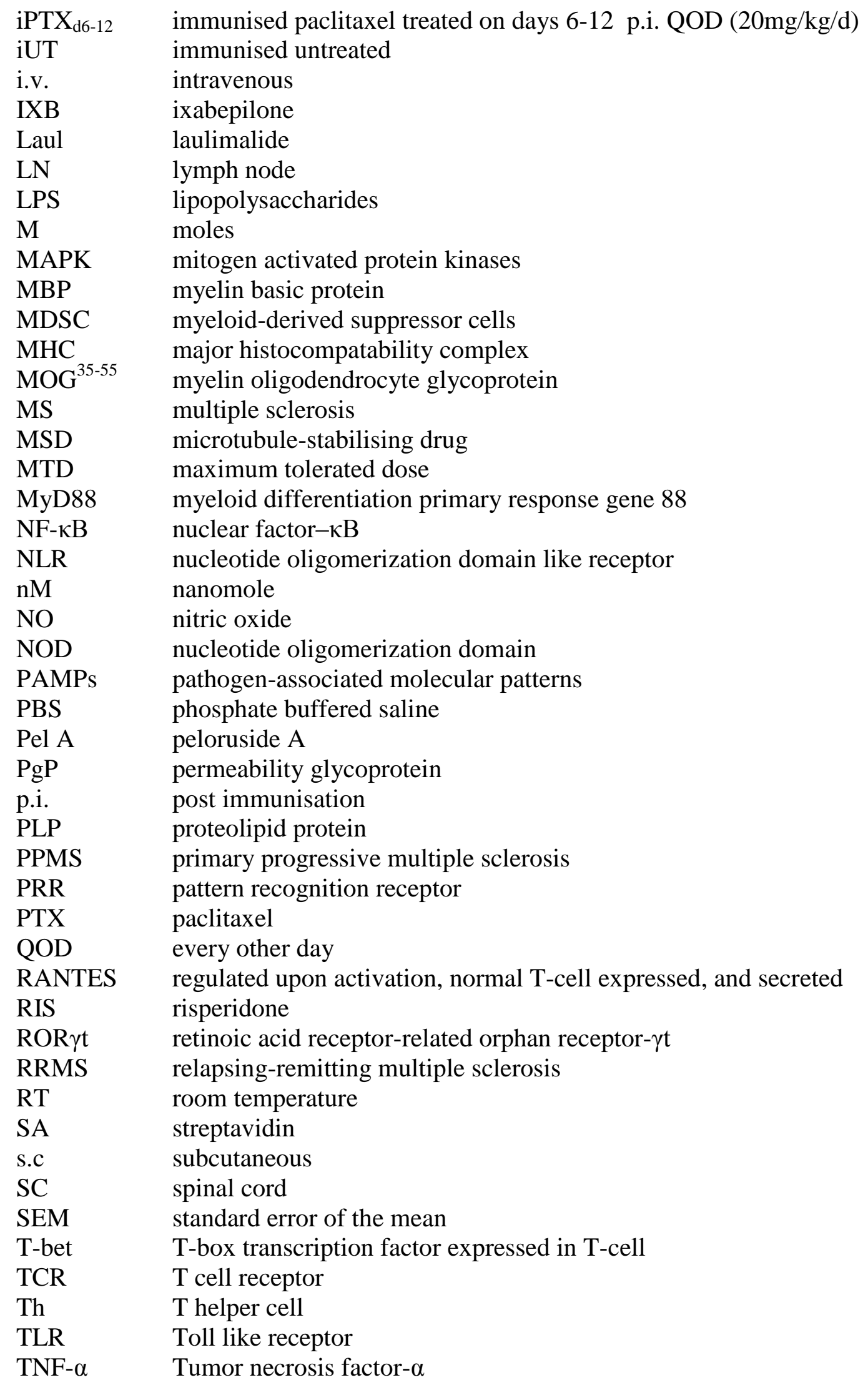




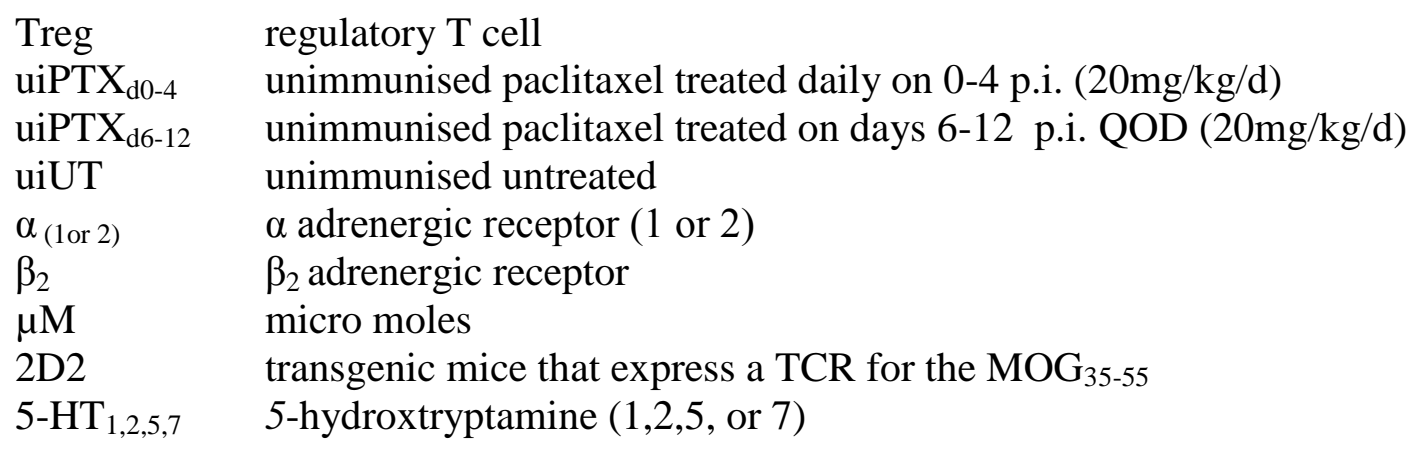




\section{Chapter 1:}

\section{General Introduction}




\subsection{The immune system}

The principle function of the immune system in all organisms is to differentiate self and non-self and thus, protect the host from potentially harmful pathogens. The mammalian immune system is a complex collection of cells and cellular processes that are commonly divided into two complementary arms: the innate and the adaptive. The innate immune system provides a rapid response and acts as a first line of defence, but it does not form immunological memory or mount antigen-specific responses. In contrast, adaptive immune responses are slower to develop but can provide a more comprehensive and targeted response. Crucial characteristics of a successful adaptive immune response are the recognition of previously encountered pathogens (i.e. memory) and the ability to respond to specific antigens.

In the process of identifying non-self, it is critical to avoid initiating an immune response to self. Therefore, the mammalian immune system has developed a multifaceted system of regulatory mechanisms to maintain immune tolerance to self. Failures in these processes can lead to autoimmune diseases, whereby the immune system attacks the body's own cells. Multiple sclerosis (MS) is considered to be one of many autoimmune diseases in which the discrimination between self and non-self has become confused. MS, like other autoimmune diseases is extremely difficult to treat, and there is an increasing demand for more effective treatment options. The current study uses experimental models to explore potential ways to interfere with the autoimmune responses associated with MS, in the hope more successful treatments can be developed.

This general introduction provides a background on the immune system and MS. Pathogenesis of the disease and current treatments are discussed and experimental methods for which novel treatments can be developed are introduced. More specific information on disease processes and the compounds used to target these are introduced in the subsequent results chapters. 


\subsection{Innate immune response}

Components of the innate immune system include physical barriers, such as skin and mucosal membranes, soluble proteins (eg. complement) as well as specific immune cells (Janeway et al., 2002). The cells of the innate immune system include mast cells, neutrophils, natural killer (NK) cells, basophils, eosinophils and phagocytic cells (Turvey et al., 2010). To distinguish non-self, the innate immune system utilises a number of cellexpressed and soluble factors that can recognise pathogen-associated molecular patterns (PAMPs) (Medzhitov et al., 1997). These molecules are conserved structures (such as specific carbohydrate or DNA motifs) that are expressed by bacteria, viruses, yeast or protozoa but not by human cells. Recognition of PAMPs by cells of the innate immune system occurs though pattern recognition receptors (PRRs). PRRs cover a wide range of receptor types including lectin binding receptors, complement receptors, scavenger receptors, NOD-like receptors and toll-like receptors (Meylan et al., 2006; Siamon, 2002). Activation of PRRs results in downstream signalling though nuclear factor- $\mathrm{kB}(\mathrm{NF}-\mathrm{\kappa B})$, mitogen activated protein kinases (MAPKs) and type I interferons (i.e IFN- $\alpha$ and IFN- $\beta$ ), which together lead to inflammatory and antimicrobial or antiviral responses (Akira et al., 2006).

An important class of PRR are the Toll-like receptors (TLRs). Signalling though TLRs triggers innate immune responses as well as providing the necessary signals for an adaptive immune response. Within humans 10 TLRs (TLR 1-10) have been identified; while 12 functional TLRs have been found in mice (Kawai et al., 2010). Each TLR has unique functions in PAMP recognition and across the 10 known TLRs in humans, multiple PAMPS from bacteria, fungi, viruses and protozoa can be recognised (Akira et al., 2006). TLR induced signalling results in the rapid activation of transcription factors that promote the expression of pro-inflammatory cytokines, chemokines and co-stimulatory molecules (Janeway et al., 2002).

There are multiple innate immune cell types that can respond to PAMPs and other pathogenic signals. Responses from these cells can result in the direct destruction of pathogens as well the recruitment of additional immune cells. Neutrophil granulocytes are 
one of the innate immune system's main effector cells involved in the destruction of invading pathogens and the containment of infections. Neutrophils can engulf and kill micro-organisms though phagocytosis and release of highly reactive antimicrobial molecules (Nathan, 2006; Segal, 2005). Neutrophils can also play an important role in recruitment of macrophages and dendritic cells to the site of tissue injury of infection (Nathan, 2006). Basophils, another type of granulocyte, are particularly important cellular responders to parasites. They respond rapidly through the release of cytokine and leukotrienes and are thought to be involved with the initiation of Th2 type adaptive immune responses (Karasuyama et al., 2011; Min, 2008). Eosinophils, like basophils, also have a role in immune response to parasites and can release cytotoxic granules, inflammatory cytokines and leukotrienes (Munitz et al., 2004; Shamri et al., 2011). They also can assist in tissue remodelling (Hogan et al., 2008).

Mast cells respond quickly to PAMP stimuli or antibody cross linking and upon activation they can release granuals containing chemokine, cytokine and lipid based mediators (Marshall, 2004). A key function of mast cells is enhancing the speed and magnitude of immune response by recruiting other innate and adaptive immune cells, as well as causing local vasodilatation through the release of histamine and serotonin (Abraham et al., 2010).

NK cells represent a type of cytotoxic lymphocyte that can recognise and kill virus infected cells or tumour cells through the release of perforin and granzymes (Moretta et al., 2008). NK cells also contribute to defense from certain parasites and bacteria and are an important source of inflammatory cytokines such as IFN- $\gamma$ and TNF- $\alpha$ (Bryceson et al., 2006) They also can have a immune regulatory role through interactions with APC and T cells (Vivier et al., 2008)

Monocytes and macrophages, which are found in blood and other tissues respectively, have numerous roles in the innate immune response. As well as directly killing pathogens they recruit additional immune cells and secrete inflammatory cytokines such as IL-1, IL-6, TNF- $\alpha$ and IL-12 (Parihar et al., 2010). Like macrophages, dendritic cells (DCs) respond to PAMPs by expressing inflammatory cytokines, can phagocytose pathogens and recruit 
other cells of the innate immune system (Steinman et al., 2006). DCs and macrophages also provide an important link between the innate and adaptive immune systems by presenting pathogen-derived antigens along with activation signals to cells of the adaptive immune system (Janeway et al., 2002; Steinman et al., 2006).

\subsection{Adaptive immune response}

Adaptive immune responses are much slower to develop than those of the innate immune system; however once developed, these responses can more specifically target invading pathogens. Additionally, due to the formation of immunological memory, a subsequent reencounter of a particular pathogen can result in a rapid and strong immune response. The adaptive immune system includes $\mathrm{B}$ and $\mathrm{T}$ lymphocyte responses and can be divided into humoral and cell-mediated immune responses, respectively.

\subsubsection{B cells}

B cells constitute the humoral arm of the adaptive immune response. The primary role of B cells is the production of antibodies, however they also secret cytokines and can act as APCs (Lund et al., 2010). B cell-produced antibodies function in three complementary ways: binding and neutralising pathogens or toxins, opsonising and mediating pathogen destruction via phagocytic cells, and activating complement (Casadevall et al., 2004). Following $\mathrm{B}$ cell receptor recognition of a specific antigen, and under the influence of $\mathrm{T}$ cell signaling, naïve B cells undergo a process of differentiation and proliferation (Batista et al., 2009). Following activation, B cells can also undergo a process of antibody class switching and somatic hypermutation, leading to improved antigen-specificity and changes in the isotypes that are produced (Li et al., 2004; Manis et al., 2002). Most mature B cells develop into plasma B cells, which produce large quantities of antibody; while a small number will form memory B cells, which are long lived and respond quickly to subsequent antigen challenge (McHeyzer-Williams et al., 2005). 


\subsubsection{Antigen presenting cells}

In contrast to $\mathrm{B}$ cells, which can recognise antigen directly via antibody, $\mathrm{T}$ cells only recognise antigen via their TCR when it is presented by an APC on surface-expressed major histocompatibility complex (MHC) molecules. There are two major mechanisms of antigen presentation, which occur via MHC molecules or human leukocyte antigens (HLA) in humans. Endogenous antigen derived from both host cells and intracellular viruses or bacteria are presented via MHC I molecules, whereas exogenous antigens from external pathogens are expressed on MHC II molecules (Jensen, 2007). The process of antigen presentation via the endogenious pathway involves the digestion of proteins into peptide fragments within the proteasomes of cells (Vyas et al., 2008). These peptide fragments are then attached to MHC I molecules which are expressed on the cell surface. Presentation of exogenous proteins on MHC II occurs following digestion of endocytosed proteins by proteases within the endosomes (Vyas et al., 2008). However, in some circumstances cross presentation can occur whereby extracellular antigens from the endosome are loaded onto MHC I molecules (Vyas et al., 2008). Antigen presentation on MHC I occurs in nearly all cell types, while antigen presentation via MHC II is mostly limited to professional APCs (Jensen, 2007). Professional APCs include dendritic cells, macrophages, B cells and thymic epithelial cells (Jensen, 2007). However MHC II antigen presentation can also occur via other cell types such as vascular endothelial and intestinal epithelial cells under specialised circumstances (Choi et al., 2004; Hershberg et al., 2000).

Interactions between antigen expressing MHC molecules and T cell receptors (TCR) are crucial in the induction of cellular based adaptive immune responses. Along with antigen and MHC, APC provide other signals necessary for subsequent activation and development of $\mathrm{T}$ cell responses, and the success of the subsequent immune response is dependent not only on the specific recognition of the antigen, but also the presence of a number of adaptor proteins and co-stimulatory signals. The complex nature of these interactions allows for fine tuning of the immune response as well providing a regulatory mechanism with which to inhibit the development of non-specific or self reactive $\mathrm{T}$ cell responses. 


\subsubsection{T cells}

$\mathrm{T}$ cells are thymus-derived cells that are crucial for effective cellular based adaptive immune response. There are two distinct types of $\mathrm{T}$ cell lineages, that can be distinguished by their expression of $\alpha \beta$ or $\gamma \delta$ TCR (Ciofani et al., 2010). $\gamma \delta$ T cells represent a small subset of $\mathrm{T}$ cells with both innate and adaptive immune characteristics (Bonneville et al., 2010; Ciofani et al., 2010). $\alpha \beta$ T cells make up the vast majority of $\mathrm{T}$ cells and can be broadly divided into two distinct cell types, $\mathrm{CD}^{+}$cytotoxic $\mathrm{T}$ cells which recognise antigens expressed by $\mathrm{MHCI}$ and $\mathrm{CD}^{+} \mathrm{T}$ helper cells which recognise antigen expressed by $\mathrm{MHCII}$. Although $\mathrm{CD}^{+} \mathrm{T}$ cells and $\mathrm{CD}^{+}$cells are conventionally defined as helper and cytotoxic T cells, respectively, it should be noted that these definitions relate to the major but not exclusive role of these cells.

\subsubsection{CD8 Cytotoxic T cells}

The main function of $\mathrm{CD}^{+}$cytotoxic T cells (CTLs) is to identify and directly kill infected or abnormal cells (Choy, 2010). Recognition of a foreign antigen expressed on the surface of any cell within the body makes it a target for CTL-mediated destruction. This destruction occurs primarily though the release of granules containing perforin and granzymes or though Fas-Fas ligand (CD95/CD95L) interactions. Although the mechanism is not quite clear, perforin facilitates entry of granzymes into the target cell. Granzyme is a serine protease that initiates cell death though a number of pathways including caspase activation (Voskoboinik et al., 2006). Similarly, the ligation of CTLexpressed Fas ligand to Fas on a target cell leads to caspase-mediated apoptosis (Krammer, 2000). The release of cytokines, such as IFN- $\gamma$ and TNF- $\alpha$, from CTL can also assist in the destruction of target cells, as well as inducing recruitment of other effector cells (Harty et al., 2000). With their capacity to directly kill cells, CTL have an important role in controlling viral infections.

\subsubsection{CD4 T helper cells}

In contrast to $\mathrm{CD} 8^{+} \mathrm{CTLs}$, the main function of $\mathrm{CD}^{+}{ }^{+} \mathrm{T}$ helper cells is to activate or direct other immune cells to generate an appropriate immune response. $\mathrm{CD}^{+} \mathrm{T}$ cells are essential for $\mathrm{B}$ cell class switching, inducing most but not all $\mathrm{CD}^{+} \mathrm{T}$ cell responses and enhancing 
or controlling macrophage responses. Formerly, naïve $\mathrm{CD}^{+} \mathrm{T}$ lymphocytes were thought to differentiate into distinct subsets T helper 1 (Th1) or T helper 2 (Th2) (Mosmann et al., 1986); however in recent years the Th1/Th2 dichotomy has been reassessed and expanded to include other subsets including Th17 and regulatory T cells (Korn et al., 2009). All of these $\mathrm{CD}^{+}$Th subsets are defined by their cytokine profiles, expression of specific transcription factors, and their functional impact on other immune cells.

Th1 cells develop following concurrent interaction with APC and IL-12 signalling and can be identified by expression of the transcription factor, T-bet (Jenner et al., 2009). Th1 cells are the classical inflammatory $\mathrm{T}$ helper linage, producing cytokines that include IFN- $\gamma$, TNF- $\alpha$, and IL-2 (Abbas et al., 1996; Mosmann et al., 1986). These cells are important for clearance of intracellular pathogens and signalling by Th1 cells induces a primarily cell mediated immune response. In contrast Th2 cells play a major role in directing immune defenses against extracellular pathogens and inducing humoral immune responses. Th2 cells produce cytokines such as IL-4, IL-5 and IL-13 and can be identified by the transcription factor GATA3 (Zhu et al., 2006). Th17 cells differentiate under the influence of IL-6 and TGF- $\beta$ and require IL-23 to stabilise their phenotype. They can be identified by the expression of the transcription factor ROR $\gamma \mathrm{t}$ and they support neutrophil-mediated inflammation (Ivanov et al., 2006). Th17 cells primarily express IL-17, IL-21 and IL-22 and appear to have a role in defending against extracellular bacteria and fungi (Louten et al., 2009).

More recently further division of the $\mathrm{T}$ helper linage has been proposed, with IL-9 producing Th9 cells and IL-22 producing Th22 being suggested as distinct subsets (Dardalhon et al., 2008; Eyerich et al., 2009; Veldhoen et al., 2008). Additionally, the concept that $\mathrm{T}$ cells commit to a distinct $\mathrm{T}$ cell linage may need revising, as more understanding is gained on the plasticity of specific T cell subsets (O'Shea et al., 2010). As novel cellular markers are identified and the ability to differentiate between subtle cellular differences is enhanced, it is likely further classification will develop. However, within the context of MS, the present focus of research has primarily been on the 
Th1/Th17 inflammatory pathways and the regulation of these by regulatory $\mathrm{T}$ cells (Tregs) and Th2 cells.

\subsubsection{Regulatory $T$ cells}

Unlike the other cell types mentioned Tregs are thought primarily to regulate the activity of the immune response and participate in the maintenance of immune tolerance (Sakaguchi et al., 2008). They can be identified by their high expression of the IL-2 receptor (CD25) and the transcription factor Foxp3 (Sakaguchi, 2001). A number of different types of Tregs have been described; however the main distinction is of those 'naturally' arising from the thymus and those that are 'induced' from naïve T cells within the periphery (Campbell et al., 2011; Sakaguchi et al., 2006). Although the majority of Tregs are technically autoreactive in the sense that they express TCR that recognise self-antigen (Picca et al., 2006) these cells act to suppress other T cell subsets though activation of Foxp3 dependent regulatory processes, the induction of regulatory cytokines such as IL-10, regulation of cytokine levels (e.g. IL-2), or through direct cell to cell interactions (Campbell et al., 2011; Li et al., 2008; Tang et al., 2008; Vignali et al., 2008).

\section{4 $\mathrm{T}$ cell activation}

The specificity of the adaptive immune response is provided by a system in which each naïve T cell carries TCRs specific for a particular antigen. The membrane bound $\alpha$ and $\beta$ chains of the TCR each contain variable regions which are formed by the recombination of gene segments (VDJ) during T cell development in the thymus (Smith-Garvin et al., 2009). This process provides a huge array of potential antigen receptors. The variable regions interact with antigen expressed on MHC molecules and are critical in the antigen recognition process. In addition to the TCRs, T cells express either CD8 or CD4 coreceptors, which recognise conserved regions of MHC I or MHC II respectively. While the TCR/MHC interaction supports antigen-specific recognition, the TCR requires the CD3 complex to generate the signaling cascades that lead to gene transcription and $\mathrm{T}$ cell activation (Smith-Garvin et al., 2009). For successful naïve T cell activation, signaling via co-stimulatory molecules has to occur in concurrence with TCR activation. 
The most well characterised co-stimulatory molecules are CD80 (B7.1) and CD86 (B7.2) which are expressed on APCs and interact with T cells though CD28 or cytotoxic T lymphocyte associated protein 4 (CTLA-4; CD152) (Merwe et al., 2003) The functional difference between CD80 and CD86 still remains unclear; however, ligation of either of these molecules with CD28 promotes T cell activation (Sansom et al., 2003). In contrast, CD80 or CD86 interactions with CTLA-4 negatively regulates T cell activation. and although CTLA-4 has higher affinity for CD80 and CD86 than CD28, unlike CD28, it is not constitutively expressed (Alegre et al., 2001). CTLA-4 acts to limit the immune response or inhibit $\mathrm{T}$ cell activation and is highly expressed by Tregs (Sakaguchi et al., 2009). Expression of CTLA-4 can also be up-regulated on effector T cells following TCR engagement (Jain et al., 2010). In addition to CD80/86, other co-stimulatory molecules include (but are not limited to) CD40, inducible co-stimulator (ICOS) and PD-1. Interaction of CD40 with CD40L (CD154) on T cells results in an up-regulation of MHCII and production of inflammatory cytokines (Suttles et al., 2009). CD40 is also an important co-stimulatory molecule for the activation of antigen-specific $\mathrm{T}$ cell responses (Grewal et al., 1998). ICOS is involved in inducing effector T cell responses and initiating the up-regulations of chemokine receptor expression, which is necessary for the development of effective humeral immune responses (Nurieva et al., 2009; Sharpe, 2009). In contrast, PD-1 interaction acts to induce tolerance and regulate $\mathrm{T}$ cell activation (Nurieva et al., 2009).

\subsection{Immune tolerance}

After successful V(D)J recombination during T cell development, a single cell will only express one unique receptor. This recombination process allows a relatively small number of genes to generate a vast number of unique antigen receptors. However, as this process can result in receptors that are non-functional (i.e. cannot recognise self $\mathrm{MHC}$ ) or recognise self-antigens, a number of selective steps are used to identify and remove nonfunctional or self-reactive cells. These processes are referred to as immune tolerance. 
Central immune tolerance is the main mechanism by which self-reactive $\mathrm{T}$ cells are eliminated. This process occurs in the thymus, where immature $\mathrm{T}$ cells go through both a positive and negative selection process. During positive selection only $\mathrm{T}$ cells that can successfully interact with MHC molecules are allowed to survive, ensuring that only functional T cells are positively selected (Starr et al., 2003). On the other hand, negative selection provides a screening mechanism to identify and remove $\mathrm{T}$ cells that are selfreactive. This process involves the expression of many but not all self-antigens within the thymus by thymic APC. Any T cell that reacts strongly to one of these self-antigens is not allowed to progress and is deleted (Palmer, 2003). If however, a T cell possesses weak to modest affinity for a self-antigen it is driven to express FoxP3 and develop into a 'natural' thymic-derived Treg.

Despite effective central tolerance, some self-reactive $\mathrm{T}$ cells may slip through the selection process and be released into the periphery and ultimately induce autoimmunity. A variety of secondary safety mechanisms, collectively referred to as peripheral tolerance, are used to stop these T cells from causing autoimmune disease. Peripheral tolerance can occur via active suppression, through regulatory cell populations such as Tregs or other direct cell to cell interactions, while passive suppression can occur though for example, a process of cell anergy (Mueller, 2010; Walker et al., 2002).

For successful naïve T cell activation, the TCR must recognise antigen expressed on MHC molecules as well as receive a second co-stimulatory signal often through CD28 (Fathman et al., 2007). Failure to simultaneously receive both signals results in T cell anergy, where by the $\mathrm{T}$ cell becomes permanently unresponsive (Fathman et al., 2007). As co-stimulatory molecules are up-regulated on APC after ligation of PRR by microbial products, it is unlikely that co-stimulatory molecules will be expressed under normal conditions when primarily self-antigens are presented by APC. Thus, it is believed that in this case, autoreactive naïve $\mathrm{T}$ cells recognising antigen in the absence of co-stimulation will become anergic. 
Active forms of peripheral tolerance are mediated by cells directly intervening in the activity of another immune cell. This can take the form of cell surface expressed molecules such as PD-1 or CTLA-4, which when ligated can transmit inhibitory signals to $\mathrm{T}$ cells expressing these receptors (Alegre et al., 2001). There are also specific cell types that appear to act as regulators of the immune system, these include Tregs and myeloid-derived suppressor cells (MDSCs) and certain macrophage subsets. (Gabrilovich et al., 2009; Sakaguchi et al., 2008; Zorro Manrique et al., 2011). Tregs can suppress autoreactive cell responses through multiple mechanisms including co-stimulatory blockade, impairing $\mathrm{T}$ cell/APC interactions and though secretion of cytokines such as IL-10 and IL-35 or proapoptotic factors such as granzyme (Chaturvedi et al., 2011; Collison et al., 2007; Tang et al., 2008). MDSCs can alter cell activation through depletion of essential amino acids, production of reactive oxygen or nitrogen species and through cell to cell interactions (Condamine et al., 2011; Gabrilovich et al., 2009). Additionally certain macrophage phenotypes act in a regulatory manner by secretion of cytokines such as IL-10, downregulation of co-stimulatory molecules and inflammatory cytokines (Anderson et al., 2002b; Tierney et al., 2009; Zorro Manrique et al., 2011).

There are certain tissues of the body, such as the eyes, testes and CNS that are less immunologically reactive than the rest of the body. These areas are maintained in what has traditionally been referred to as 'immune privileged' states though a number of active and passive toleragenic process (Streilein et al., 2000). In addition to the tolerance mechanisms mentioned already, cells within these tissues can have decreased MHC expression and secrete increased levels of regulatory cytokines and inhibitors of migration (Hong et al., 1999; Rosenzweig et al., 2011; Streilein et al., 2000). This combined with physical barriers and reduced lymphatic drainage reduces the immune surveillance and reactivity of immune cells within these tissues.

If the maintenance of immune tolerance fails, immune cells reactive to self-peptides can elicit an inflammatory immune response. This can lead to the development of autoimmune diseases, such as MS. 


\subsection{Multiple sclerosis}

MS is a chronic inflammatory disease, mediated by immune cells targeting the myelin sheaths that surround nerve axons. The damage caused is characterised by plaque formation and CNS dysfunction. The symptoms of MS are mainly related to altered nerve conduction which results in muscular neuropathy/spasticity, altered pain and tactile sensation, loss of bowel or bladder control and potential cognitive dysfunction (Lutton et al., 2004). There is much heterogeneity in disease presentation and pathology, particular disease symptoms can be chronic or transient and range from mild to severe (Lucchinetti et al., 2000). The diversity in disease symptoms, clinical progression and the immune responses that underlie these, adds to the complexity of both diagnosing and treating MS.

There are two main forms of multiple sclerosis, relapsing-remitting (RRMS) and primary progressive (PPMS) (Lubin et al., 1996). Relapsing-remitting is the most prevalent with 85-90\% of all cases initially falling within this category (Sospedra et al., 2005a). Many relapsing-remitting cases develop into a secondary-progressive form within five to ten years of disease onset (Steinman, 2001). The usual age of onset ranges between 20-40 years of age, however MS can be diagnosed within the first decade of life (Banwell et al., 2007). Although MS is not usually considered a lethal disease, mortality can result in some rare circumstances, when brainstem lesions occur (Barnett et al., 2004). Additionally, it has been estimated that MS sufferers have an overall 5-10 year reduction in life expectancy compared to the general population (Ragonese et al., 2008). Women are more susceptible to MS than men, with a prevalence ratio of around 2.5:1 in RRMS, however this gender bias is not evident in PPMS (Keegan et al., 2002; Willer et al., 2003).

\subsubsection{Disease aetiology}

Despite an extensive amount of research no definitive causative factors for MS have been identified, although epidemiological studies have identified a variety of potential risk factors including diet, smoking, infectious agents, geographical location and genetics (George C, 2008; Handel et al., 2011; Nielsen et al., 2007). Several large studies have explored genetic risk factors for MS. Genes that have consistently been recognised to confer risk are those associated with MHC II alleles, these include HLA-DR and HLA-DQ 
(Consortium, 2011; Dyment et al., 2005; Dyment et al., 1997). The concordance rate of MS in monozygotic twins is around $25 \%$ in high prevalence countries (Mumford et al., 1994; Willer et al., 2003). Overall concordance in dizygotic twins is around 3-5\% and nontwin siblings is around 0.1-0.4\% (Mumford et al., 1994; Sadovnick et al., 1996). However, if males are analysed separately the concordance between dizygotic and monozygotic twins is similar, which highlights the important role sexual dimorphism has in MS (Willer et al., 2003). The relatively low concordance rates and minimal gene associations suggest non-genetic factors are more significant determinants in the aetiology of MS.

It has been postulated that viral agents such as human herpes virus 6, JC virus and Epstein Barr virus (EBV) could have a role in causing MS, however strong causative associations between these viruses and the development of MS have yet to be shown (Donald H, 2005; Noseworthy, 1999). Most of the research investigating the potential link between MS and viruses has focused on EBV and its clinically defined condition, infectious mononucleosis. Studies on large cohorts of MS patents have shown an approximate 2-fold increase in the risk of MS development in people that had previously had infectious mononucleosis (Lucas et al., 2011; Nielsen et al., 2007). However EBV is estimated to infect over $90 \%$ of the world's population, with around $40 \%$ of adolescent or adults experiencing infectious mononucleosis (Ascherio et al., 2011; Macsween et al., 2003). The fact that EBV infection is so wide spread suggests that any role this infection has in MS etiology is only a small contributing factor.

A correlation between latitude and MS has been reported with increased prevalence at higher latitudes. A meta-analysis of over 300 peer-reviewed studies has recently demonstrated a significant correlation between latitude and MS prevalence, although this correlation is not absolute since some areas, such as Italy and a few Scandinavian countries, do not follow the overall trend (Simpson et al., 2011). This correlation between latitude and MS has been postulated to be associated with reduced sunlight hours at higher latitudes and the subsequent reduction in vitamin D production (Pierrot-Deseilligny et al., 2010). 
The vitamin D hypothesis has gained momentum as a credible explanation for the latitudinal variance. Low plasma levels of Vitamin D correlate both to higher latitude and to increased risk of MS (Handel et al., 2010). While most vitamin D is synthesised during exposure to sunlight (ie. UVB radiation), certain foods can also provide a source of vitamin D (Ascherio et al., 2010). In some populations that consume large quantities of vitamin D rich food, the prevalence of MS is lower than would be predicted by latitudinal variance (Ascherio et al., 2010). Additionally, within a large 30 year prospective study, supplementation with vitamin D was associated with reduced MS incidence risk (Munger et al., 2004).

Another potential explanation for some of the geographical variation could be associations with infectious organisms that are more prevalent in warmer climates (Fleming et al., 2006). The low incidence of MS in tropical regions relative to colder climates has been suggested to be associated with parasitic load of the populations living in the respective regions (Correale et al., 2011). Epidemiological studies have demonstrated that areas with high autoimmune disease prevalence exhibit an inverse relationship to areas of high parasitic load (Bach, 2002; Zaccone et al., 2006). This inverse relationship also occurs with MS, as countries with a high prevalence of helminth infections have low MS incidence rates (Fleming et al., 2006; Philippe, 2009). Additionally longitudinal studies in the West Indies have found incidence of MS increased over time while parasitic infections have concurrently decreased (Cabre et al., 2005). Moreover, patients with RRMS who are subsequently infected with helminths have a reduced relapse rate and disease progression suggesting that parasites may have a protective effect on the autoimmune process (Correale et al., 2007; Correale et al., 2008). On the strength of this evidence and of studies in animal models, a phase I clinical trial was run and demonstrated the potential of helminths to treat RRMS and a phase II trial is currently underway (Fleming et al., 2011).

Like the heterogeneity in disease symptoms and pathology, it is likely that the factors which cause MS may vary and be specific to the individual. The epidemiological research discussed above indicates that no clear single causal factor has been established. This suggests that multiple culminating factors are required to induce MS and that pinpointing a 
particular cause for this disease may not be possible. Therefore development of treatments for MS needs to rely on understanding the immunology and pathology of disease progression and ways to alter these processes.

\subsubsection{Multiple sclerosis immunology}

MS is thought to be primarily driven by autoreactive $\mathrm{CD}^{+} \mathrm{T}$ cells targeting myelin proteins, such as myelin basic protein (MBP), proteolipid protein (PLP) and myelin oligodendrocyte glycoprotein (MOG) (Steinman, 1996). However, the mere presence of myelin reactive $\mathrm{T}$ cells is not sufficient to induce disease, as these autoreactive cells can be found within the blood of both MS patients and within the general population (Ota et al., 1990; Pender et al., 1996). Nevertheless autoreactive cells from MS patients do become activated more readily than cells from healthy individuals and these cells occur in higher numbers, suggesting clonal expansion of these self-reactive cells occurs within MS patients but not the general population (Bar-Or et al., 1999). While MS is considered to be a $\mathrm{CD}^{+} \mathrm{T}$ cell mediated disease, many other cell types have a role including local glial cells, $\mathrm{CD}^{+} \mathrm{T}$ cells, macrophages, mast cells and B cells (Friese et al., 2009; Genain et al., 1999; Henderson et al., 2009; Sospedra et al., 2005b).

What initiates the underlying immune response is not known, however it is generally believed that the induction of the autoimmune cascade that ultimately leads to MS occurs in the periphery (McQualter et al., 2007). It has been suggested that these initial events could be caused by molecular mimicry, whereby myelin reactive $\mathrm{T}$ cells become activated after encounters with bacterial or viral antigens that have a similar molecular sequence or structures to that of myelin based antigens (Libbey et al., 2007). Although molecular mimicry could potentially provide an explanation for the heterogeneity of disease onset and pathology, as well as the regional differences in incidence, there is very little evidence to support this theory.

It has also been found that Tregs, which usually suppress the activation of autoreactive T cells, are often functionally deficient in MS patients (Haas et al., 2005; Viglietta et al., 2004). While Tregs are found in MS patients they often occur in lower numbers and have 
impaired regulatory activity (Costantino et al., 2008; Viglietta et al., 2004). It is possible that impaired Treg function allows for expansion of autoreactive $\mathrm{T}$ cell responses and the induction of an autoimmune response.

From the periphery, the myelin reactive T cells must traffic into the CNS to induce disease pathology. Because the CNS is an immune privileged area, any immune cell trafficking that occurs is tightly regulated by the specialised endothelial cells that make up the blood brain barrier (BBB)(Carson et al., 2006). For autoreactive T cells to enter into the CNS, it is believed that some perturbation of this regulatory barrier must occur (Larochelle et al., 2011). In MS patients, breakdown and permeability of the BBB is apparent, allowing for immune cell infiltrations into the CNS (Minagar et al., 2003; Stone et al., 1995). This infiltration can occur though the secretion of inflammatory cytokines, chemokines and reactive oxygen species, which results in increased permeability and immune cell migration (Wilson et al., 2010). These processes are also aided by the destruction of extracellular matrix components by macrophage- and $\mathrm{T}$ cell-derived matrix metalloprotenases (Minagar et al., 2003). Immune cell transit from the periphery into the CNS is clearly a crucial factor in the disease process since inhibiting this migration is an effective way of treating MS (Hutchinson, 2007). Once autoreactive T cell have gained access to the CNS and recognised myelin antigens (present within the CNS white matter), these cells can recruit further cells and cause development of an inflammatory lesion. Although most of the lesions occur in white matter, grey matter (i.e. neuronal tissue) lesions can often also be detected, indicating that tissue damage occurring during MS is not solely limited to myelinated areas (Stadelmann et al., 2008). Reactive oxygen and nitrogen species, complement, antibodies, microglia, $\mathrm{CD}^{+}, \mathrm{CD}^{+}$, neutrophils, $\mathrm{B}$ cells and macrophages are all thought to have a role in tissue destruction (Disanto et al., 2011; Frohman et al., 2006; Gay et al., 1997; Genain et al., 1999). However, while CD4 ${ }^{+}$cells are crucial for initiation of disease, macrophages and $\mathrm{CD}^{+} \mathrm{T}$ cells are the most prevalent within lesions, suggesting these cell may have a significant role in causing the damage (Disanto et al., 2011; McQualter et al., 2007). After the initial inflammatory influx, over time the inflammation will usually recede and depending on the severity of damage, the 
myelin may regenerate or scars may be formed and irreversible axonal damage could have occurred (Frohman et al., 2006).

Due to the obvious limitations in studying immune responses and disease pathology in MS patients, most of the knowledge of underlying immunology of MS within the CNS has been gained from post-mortem studies or is inferred from animal models of MS (discussed below).

\subsubsection{Current treatment options}

There are currently eight food and drug administration (FDA) approved disease modifing therapies, which are mainly used in the treatment of relapsing-remitting MS. None of the current drugs are effective for primary-progressive disease (Leary et al., 2005). While all MS therapies have distinct mechanisms of actions, most have a limited efficacy rate of between $30-60 \%$ for reducing relapse rates or disability progression (Confavreux et al., 2006; DeAngelis et al., 2008; Sorensen, 2007). The exception is Natalizumab which can provide $40-70 \%$ reductions in disease progression, but is also associated with progressive multifocal leukoencephalopathy (PML), a potentially fatal brain disease (Hutchinson, 2007).

The first drug introduced to the market was Betaferon/Betaseron ${ }^{\circ}$, an interferon- $\beta 1 \mathrm{~b}$ cytokine in 1993. Two further interferon-based cytokine treatments, Avonex $®$ and Rebif $®$, which are both recombinant interferon- $\beta$ 1a, followed in 1996 and 2002, respectively. These drugs potentially work by altering the immune response through a number of mechanisms, including preventing up-regulation of MHC II expression on antigen presenting cells (APC), causing down-regulation of co-stimulatory molecules and inhibiting cell migration across the BBB (Dhib-Jalbut et al., 2010; Nakatsuji et al., 2007). interferon- $\beta$ is administered 1-3 times a week by subcutaneous or intramuscular injection and despite inducing flu-like symptoms it is generally well tolerated (Limmroth et al., 2011). However, the efficacy of interferon- $\beta$ can substantially diminish as the body begins to develop antibodies against the recombinant protein (Giovannoni et al., 2002). 
In 1996, glatiramer acetate (GA) was approved under the name Copaxone ${ }^{\circ}$ and is currently the most prescribed treatment in the United States (Boster et al., 2011a). It consists of short random length polypeptide sequences constructed of tyrosine, glutamic acid, alanine and lysine residues in similar ratios to that found in myelin basic protein (Schrempf et al., 2007). GA is administered every day by subcutaneous injection and is considered by some to have the mildest adverse reactions, which include injection site inflammation, chest tightness and anxiety (Boster et al., 2011b; Ruggieri et al., 2007). Like interferon- $\beta$ treatments, patients can develop antibodies to GA, however, anti-GA antibodies do not appear to have much of an impact on treatment efficacy (Karussis et al., 2010; Teitelbaum et al., 2003). GAs mechanism of action is not fully understood, however it is known to be presented by MHC II on APCs and cause alterations in T cell effector responses (Ruggieri et al., 2007). In animal studies as well as human studies, GA has also been shown to have a variety of other effects including Th2 response biasing induction of Tregs, and altering monocyte and microglial activation (Aharoni et al., 2010; Aharoni et al., 1997; Toker et al., 2011; Weber et al., 2007). How GA mediates these wide ranging effects is, however, unclear.

Mitoxantrone, a type II topoisomerase inhibitor that is used in cancer chemotherapy, was released as Novantrone ${ }^{\circledR}$ in 2000 and is the only treatment available for secondary progressive MS (SPMS). It is an effective treatment for both RRMS and SPMS resulting in 30-60\% reductions in relapse rates and disability progression, respectively (Esposito et al., 2010; Hartung et al., 2002). Mitoxantrone is administered by intravenous infusion every few months and causes acute side effects which include nausea, vomiting and hair loss; as well as long term side effects, which include cardiotoxicity and increased risk of leukemia (Marriott et al., 2010). Due to the severity of the long term side effects, its use is limited to patients that have very active disease or have not responded to other treatment options (Marriott et al., 2010). Moreover, it has a restricted cumulative lifetime limit of 8-12 doses meaning it can only be used as a transitory treatment option (Neuhaus et al., 2006).

Natalizumab, marketed as Tysabri ${ }^{\circledR}$, is a humanised monoclonal antibody against $\alpha 4 \beta 1$ integrin (VLA-4), which is an important adhesion molecule expressed on T cells. Binding 
of $\alpha 4 \beta 1$-intergrin to the endothelial-expressed VCAM-1 is a necessary step for $\mathrm{T}$ cell migration into the CNS, and the inhibition of this process by Natalizumab has proved an effective method of decreasing MS relapses (Hutchinson, 2007). Natalizumab is administered by monthly intravenous infusions and side effects include infusion reactions, nausea, fatigue and limb swelling (Pucci et al., 2011). Additionally, three months following Natalizumab release in 2004, it was quickly withdrawn when it became apparent that it contributes to the development of the usually lethal viral disease, progressive multifocal leukoencephalopathy (PML), in some patients (Chaudhuri, 2005; Tan et al., 2010). Despite this finding, Natalizamab was re-released in 2006 due to its high efficacy rates but under strict monitoring guidelines and with the patients' knowledge that there is a 1:1000 risk of developing PML (Tan et al., 2010; Yousry et al., 2006).

Fingolimod (FTY720) is an oral treatment that was approved by FDA in 2010 (Singer et al., 2011). It is marketed under the name Gilenya® and is indicated for RRMS. Fingolimod is a fungual derivative that modulates sphingosine 1-phosphate and causes inhibition of the egress of lymphocytes from lymph nodes (Kataoka et al., 2005). This in turn prevents subsequent lymphocyte migration into the CNS; however it may also have more direct effects within the CNS (Brinkmann, 2009; Kataoka et al., 2005; Lee et al., 2010). Fingolimod is the first orally administered treatment for MS, side effects include headache, back pain and increased risk of contracting influenza (Kappos et al., 2006; Singer et al., 2011). The disease modifying effects of fingolimod diminish rapidly once the drug has been cleared from the body and thefore daily doses must be taken (Kovarik et al., 2007). Results from Phase III trials indicate that fingolimod is well tolerated and may have improved efficacy over interferon- $\beta$ in reducing relapse rates and disability progression (Cohen et al., 2010; Kappos et al., 2010).

MS is a chronic disease that can afflict patients for many decades. None of the current treatments cure the disease, and patients will spend their lives using these drugs. Additionally the cost effectiveness of the current disease modifying therapies is very low (Noyes et al., 2011). The combination of these two factors makes MS a very costly disease to treat and places a large burden on patients and health care providers. It becomes 
apparent when considering the limited scope and efficacy of current treatments that more effective options are needed. However, the variable nature of immunological and clinical disease manifestations, as well as the limited understanding of disease aetiology, makes disease specific drug targeting difficult. To overcome the heterogeneity in disease expression, future therapies will ideally provide a broad spectrum of effects, which may include the removal or tolerance of autoreactive $\mathrm{T}$ cells, the inhibition or reversal of CNS pathology and the restoration of normal immune activity.

\subsection{Animal models of multiple sclerosis}

A number of different animal models have been used to explore potential factors associated with MS. These include viral and antigen-induced encephalomyelitis, drug induced demyelination and genetically modified models. Of these models, experimental autoimmune encephalomyelitis (EAE) is the most commonly used and well defined, and has been used to develop many of the current MS treatments (Steinman et al., 2005). To induce EAE, laboratory animals are immunised with myelin peptides or proteins, which leads to an autoimmune response that mimics aspects of MS pathology. There are two main forms of EAE induction; active, which involves direct immunisation with encephalitogenic compounds and passive, which involves the adoptive transfer of primed encephalitogenic $\mathrm{CD}^{+} \mathrm{T}$ cells into naïve animals (Gold et al., 2000). Using these basic techniques, many variations have been developed to induce distinct aspects of disease presentation which emulate that of MS (Gold et al., 2006).

Mice, a species regularly used in EAE experiments, often exhibit strain specific susceptibly and pathology to a particular antigen. SJL/J mice are susceptible to MOG, PLP and MBP and exhibit a relapsing-remitting type EAE (Amor et al., 1994; Pettinelli et al., 1982; Tuohy et al., 1988). In C57BL/6 mice MOG is often used to induce EAE, which results in severe, chronic disease expression (Bernard et al., 1997). Balb/c mice have traditionally been considered more resistant to EAE (Duong et al., 1994a; Tuohy et al., 1988). While disease can be induced in Balb/c by PLP, in contrast to C57BL/6, the disease has a lower induction rate (50-60\%), milder disease symptoms, and the disease completely resolves (Greer et al., 1996; Lyons et al., 2002). The divergence in disease presentation 
between different experimental protocols or mice stains can be used beneficially to explore different aspects of pathology, relevant to MS.

There is ongoing debate on the applicability of the different animal models of MS and whether they are useful for drug development (Baker et al., 2011; Sriram et al., 2005). Many novel treatments which have shown some success within animal models have failed to translate into human therapies. However most of the current MS treatments were developed within EAE models, indicating that there is some value to this method of drug development (Steinman et al., 2005). Additionally, as no adequate alternative methods of MS drug development have been proposed, animal models currently provide the most likely pathway to identifying new treatments for MS.

Most of the experiments conducted in the current study have used C57BL/6 mice immunised with a synthetic MOG peptide comprising of amino acids 35-55, which is injected in complete Freund's adjuvant (CFA). Using this model, 80-100\% of mice develop a 'chronic progressive' disease 1-2 weeks after immunisation. Within four weeks after immunisation, partial remission of disease symptoms can be observed, but full recovery is rare. This model is the most commonly used EAE model and due to high incidence rates and robust disease expression, it provides a good platform with which to explore potential MS treatments.

\subsubsection{Immunology of EAE}

EAE is primarily a $\mathrm{CD} 4^{+} \mathrm{T}$ cell mediated disease and exhibits many of the characteristic immunological events that occur in MS. It is, however, important to keep in mind that unlike MS, for EAE to occur, the immune system has to be artificially induced to break self-tolerance and develop an autoimmune state. As mentioned above induction of EAE can be by immunisation with a combination of myelin based peptide/proteins and a strong immune adjuvant, or though the transfer of $\mathrm{T}$ cells that are already reactive to myelin antigens. In either of these systems autoreactive $\mathrm{T}$ cells have to be generated to induce disease. 
Induction of autoreactive T cells occurs following exposure to APCs that present myelin based peptides and co-stimulatory molecules to naïve $\mathrm{CD}^{+} \mathrm{T}$ cells. $\mathrm{T}$ cells that recognise these peptides undergo a process of maturation and clonal expansion that results in the development of a large pool of myelin-reactive $\mathrm{T}$ helper cells. The particular subset of $\mathrm{T}$ helper cells (eg Th1/Th2/Th17) that develops depends on the cytokine environment present during the development process. It was originally believed that EAE and MS were Th1 mediated diseases. However, the identification and characterisation of Th17 cells has led to revision of this paradigm, and it is now apparent that Th17 cells have a crucial role in EAE (Steinman, 2007).

A number of factors implicate Th17 immune responses in mediating disease pathology. EAE is exacerbated in either IFN- $\gamma$ deficient or IFN- $\gamma$ receptor deficient mice (Ferber et al., 1996b; Sabatino et al., 2008). In contrast IL-17 deficient mice or mice treated with anti-IL17 antibodies have attenuated disease expression (Hofstetter et al., 2005; Komiyama et al., 2006b). Additionally mice lacking functional IL-23 (which sustains Th17 cells) but not IL12 (which induces Th1 cells) are resistant to EAE (Cua et al., 2003). It also appears that in some situations Th1 expressed IFN- $\gamma$ can reduce Th17 encephalogenic cell numbers, while conversely an absence of IFN- $\gamma$ results in increased Th17 cells (Berghmans et al., 2011). These studies suggest that Th17 cell may have an important role in EAE disease pathology. Despite these findings, EAE is not completely ablated in the absence of IL-17 indicating that EAE is not solely mediated by Th17 cells (Komiyama et al., 2006a). Indeed this idea is supported by adoptive transfer experiments whereby purified populations of myelin reactive Th17 cells only induce mild EAE, while adoptively transferred Th1 cells result in severe disease (O'Connor et al., 2008). Collectively these results suggest a role both for Th1 and Th17 type responses in EAE.

For autoreactive $\mathrm{T}$ cells to induce disease, they must migrate from the peripheral lymphoid organs into the CNS. Studies have shown that leukocytes enter CNS in healthy individuals although in relatively low numbers and with the majority of cells being $\mathrm{CD} 4^{+}$memory $\mathrm{T}$ cells (Ransohoff et al., 2003). It is thought that these cells gain access by migration across the endothelium and epithelium associated with the choroid plexis to enter into the cerebral 
spinal fluid within the subarachnoid space (Carrithers et al., 2002; Ransohoff et al., 2003). In EAE immunised mice, the subarachnoid space is the first site within the CNS where autoreactive $\mathrm{T}$ cells are detected, suggesting this maybe the initial site of $\mathrm{T}$ cell reactivation within the CNS (Kivisäkk et al., 2009). It is thought that following reactivation, cytokine and chemokine signalling results in the recruitment of other immune cells to the CNS as well as causing up-regulation of adhesion molecules on vascular endothelial cells (Engelhardt, 2010; Goverman, 2009; Steinman, 2005).

Expression of adhesion molecules on vascular endothelial cells is crucial to the extravasion process necessary for large numbers of activated $\mathrm{T}$ cells to cross the BBB. During EAE, intracellular adhesion molecule-1 (ICAM-1) and vascular adhesion molecule-1 (VCAM-1) are up-regulated by vascular endothelial cells (Steffen et al., 1994). These bind to $\alpha 4 \beta 1$ integrin (VLA-4) and lymphocyte function-associated antigen 1 (LFA-1) which are expressed on encephalogenic T cells (Engelhardt, 2010; Engelhardt et al., 1998b). Interactions between these adhesion molecules are a crucial step in the process of $\mathrm{T}$ cell extravasations from the blood into the CNS and inhibition of $\alpha 4 \beta 1$ or VCAM1 inhibits EAE development (Engelhardt et al., 1998a; Piraino et al., 2002).

As more T cells infiltrate into the CNS and recognise myelin antigens, the inflammatory response is amplified. Cytokines (e.g. IFN- $\gamma$, IL-17, TNF- $\alpha$ ) and chemokines (e.g. CCL3, CCL20) are secreted from the infiltrating $\mathrm{T}$ cells to further enhance inflammation and recruit additional inflammatory cells including more Th cells, NK cells, macrophages, and $\mathrm{CD}^{+}$T cells (Behi et al., 2005; Engelhardt, 2010; Karpus et al., 1998; McFarland et al., 2007; McQualter et al., 2007). These cells can mediate direct damage to the oligodendrocytes and induce demyelination. $\mathrm{CD}^{+} \mathrm{T}$ cells can participate in this destruction with the release of cytotoxic molecules or by receptor mediated apoptosis, through for example granzyme B and Fas-FasL interactions, respectively (Johnson et al., 2010). Macrophages and microglia also have an important role in pathology and can cause direct tissue damage through production of MMPs, NO and reactive oxygen species (Herz et al., 2009; McQualter et al., 2007). 
However the inflammatory responses do not proceed unchecked, as most mice exhibit some form of remission. The regulation of the inflammatory process appears to be associated with Tregs, which accumulate in the CNS during the recovery phase of disease and depletion of these cells worsens disease and inhibits recovery (McGeachy et al., 2005; McNeill et al., 2007). Regulation of effector T cells by Tregs during EAE appears to be mediated by production of IL-10 and TGF- $\beta$ as well as direct cell-to-cell mediated interactions (Fletcher et al., 2010; Vignali et al., 2008; Zhang et al., 2004). However, these cells are not the only cells important in regulation of the inflammatory response and in some cases their regulatory function may be inhibited. For example, Tregs can be present during the height of inflammation but during this time, inflammatory cytokines such as TNF- $\alpha$ and IL-6 may be inhibiting Treg function or even subverting Tregs towards an inflammatory phenotype (Hawiger et al., 2009; Korn et al., 2007; O'Connor et al., 2010). This finding suggests that other cell types may have to initiate regulatory processes before Tregs can be effective. Additionally, classical pro-inflammatory cytokines such as IFN- $\gamma$ may also have a paradoxical role in regulating the magnitude and duration of inflammatory responses (Sabatino et al., 2008; Zhang, 2007). Thus both the cytokine melee as well as the cellular composition of the lesions, is likely to influence the regulatory processes necessary for remission.

In summary, EAE is clearly a complex disease that involves a large cascade of events. Overall, the disease process can be divided into three crucial steps: 1) activation and clonal expansion of myelin reactive $\mathrm{T}$ cells, 2) migration of myelin reactive $\mathrm{T}$ cells from the periphery into the CNS, 3) amplification of inflammatory responses in the CNS and development of inflammatory lesions. These three steps provide targets against which drug interventions can be directed. 


\subsection{Overall aims and objectives}

From the outset, the overall goal of this thesis was to identify potential therapies for MS, using the EAE model. As such, this thesis developed into three separate lines of inquiry. The primarily focus has been expanding previous work conducted in our laboratory investigating the use and mechanisms of actions of microtubule-stabilising drugs for the treatment of EAE. As opportunities arose, two other distinct and novel therapies; the use of an antipsychotic, risperidone and using a TLR-9/NOD2 agonist to treat EAE, were also explored. The specific details of each compound, the justifications for exploring each treatment option and specific hypotheses are introduced at the start of each chapter.

The overall aims of this thesis are:

1. To explore the use of microtubule-stabilising drugs to treat EAE.

2. To identify potential mechanisms of action by which microtubule-stabilising drugs alter disease expression.

3. To determine if the treatment efficacy of microtubule-stabilising drugs are enhanced when used together or in combination with another disease modifying agent.

4. To evaluate whether the anti-psychotic drug, risperidone, can modify EAE disease expression and inflammatory macrophage activation.

5. To investigate if a novel TLR9/NOD2 agonist, MIS416, can alter EAE disease responses. 


\section{Chapter 2:}

\section{Materials and Methods}




\subsection{Mice}

All animals were housed in the animal facilities at Victoria University of Wellington, New Zealand. Experimental protocols were approved by the Victoria University of Wellington Animal Ethics Committee (AEC) under the licenses 2002R6 and 2004R17. The animals were maintained in a temperature-regulated environment with a 12-hour light/dark cycle. Mice were kept in filter-top cages and any handling of the animals was conducted in a laminar flow workspace.

C57BL/6J (H-2b) mice were bred at the Malaghan Institute of Medical Research (Wellington, NZ), or the Biological Research Unit (Wellington School of Medicine, Wellington, NZ). Breeding stock was originally purchased from the Jackson Laboratory (Bar Harbour, ME, USA).

$\mathrm{SJL} / \mathrm{J}$ mice were bred at Victoria University of Wellington. Breeding stock was originally purchased from Animal Resource Centre (Canning Vale, WA, Australia).

$\mathrm{Balb} / \mathrm{c}$ mice were bred at Victoria University of Wellington. Breeding stock was originally obtained from Frank Brombacher (University of Cape Town, Cape Town, South Africa).

2D2 mice, which express a transgenic TCR for the $\mathrm{MOG}_{35-55}$ peptide (Bettelli et al., 2003), were bred at the Malaghan Institute of Medical Research (Wellington, NZ) or at Victoria University of Wellington. Breeding stock was originally obtained from Prof. Vijay K. Kuchroo (Harvard Medical School, Boston, MA, USA).

B6.SJL-ptprca mice, which express CD45.1 on a C57BL/6 background, were bred at the Malaghan Institute of Medical Research (Wellington, NZ). Breeding stock was originally purchased from Animal Resource Centre (Canning Vale, WA, Australia).

C57BL/6-ifngtm1Ts IFN- $\gamma$ deficient mice (Dalton et al., 1993), were bred at the Malaghan Institute of Medical Research (Wellington, NZ). Breeding stock was originally obtained from the Walter and Eliza Hall Institute of Medical Research (Melbourne, VIC, Australia). 


\subsection{Peptides}

MOG $^{35-55}$ peptide (MEVGWYRSPFSRVVHLYRNGK) was synthesised by GenScript (Piscataway, NJ, USA)

PLP $^{139-151}$ peptide (HSLGKWLGHPDKF) was synthesised by GenScript (USA)

PLP $^{180-199}$ peptide (WTTCQSIAFPSKTSASIGSL) was synthesised by GenScript (USA)

\subsection{EAE induction}

\subsubsection{C57BL/6J mice}

Eight to twelve week old mice were immunised with an emulsion consisting of $50 \mu \mathrm{g}$ MOG $^{35-55}$ peptide (Genscript, Piscataway, NJ, USA) and $500 \mu \mathrm{g}$ heat-inactivated Mycobacterium tuberculosis (Difco Laboratories, Detroit, USA) in incomplete Freund's adjuvant (Sigma, St. Louis, MO, USA). The components were emulsified prior to injecting by repeatedly passing through an $18 \mathrm{~g}$ needle until a thick homogenised emulsion was achieved. Mice were injected s.c. with $100 \mu$ in each hind flank. In addition, each mouse was injected i.p. with 200 ng pertussis toxin (Sapphire Bioscience, Redfern, NSW, Australia) in $200 \mu \mathrm{l}$ pertussis toxin buffer on days 0 and 2 p.i. as previously described (Crume et al., 2009).

\subsubsection{SJL/J mice}

Eight to sixteen week old mice were immunised with an emulsion consisting of $50 \mu \mathrm{g}$ PLP $^{139-151}$ peptide (Genscript, USA) and $500 \mu \mathrm{g}$ heat-inactivated Mycobacterium tuberculosis (Difco Laboratories, USA) in incomplete Freund's adjuvant (Sigma, USA). The components were emulsified prior to injecting by repeatedly passing through an $18 \mathrm{~g}$ needle until a thick homogenised emulsion was achieved. Mice were injected s.c. with 100 $\mu \mathrm{l}$ in each hind flank. Mice received i.p. injections of $100 \mathrm{ng}$ pertussis toxin (Sapphire Bioscience, AU) in $200 \mu$ pertussis toxin buffer on days 0 and 2 p.i. Optimisation experiments were initially conducted to ascertain the concentrations of PLP ${ }^{139-151}$ and pertussis toxin that would induce optimal relapsing-remitting EAE within the SJL/J mice 
housed at the animal facilities at Victoria University of Wellington (data not shown). The concentrations of used PLP ${ }^{139-151}$ and pertussis toxin used in the current experiments are consistent with concentrations used in previously published research (Kleinschek et al., 2007).

\subsubsection{Balb/c}

Eight to sixteen week old mice were immunised with an emulsion consisting of $100 \mu \mathrm{g}$ PLP $^{180-199}$ peptide (Genscript, USA) and $500 \mu \mathrm{g}$ heat-inactivated Mycobacterium tuberculosis (Difco Laboratories, USA) in incomplete Freund's adjuvant (Sigma, USA) (Keating et al., 2009). The components were emulsified prior to injecting by repeatedly passing through an $18 \mathrm{~g}$ needle until a thick homogenised emulsion was achieved. Mice were injected s.c. with $100 \mu \mathrm{l}$ in each hind flank. Each mouse received additional i.p. injections of $100 \mathrm{ng}$ pertussis toxin (Sapphire Bioscience, AU) in $200 \mu \mathrm{l}$ pertussis toxin buffer on days 0 and 2 .

\subsubsection{EAE disease severity rating scale}

The mice were weighed daily using a Mettler PB6000 balance (Mettler Tolodo; Columbus, $\mathrm{OH}$, USA) and monitored for any signs of disease throughout the duration of the experiment. Mice were scored for EAE using a commonly used 0-5 disease severity rating scale: $0.5=$ loss of tonicity in distal region of tail, $1=$ loss of tail tonicity; $2=$ full tail paralysis; 3 = one hind limb paralysis or severe weakness in both hind limbs; $4=$ full hind limb paralysis; and 5 = moribund (Keating et al., 2009; Tierney et al., 2009). Mice that reached an EAE score of 5 were euthanised.

\subsection{Compounds for in vivo use}

Paclitaxel (Sandoz (Ebewe), Hilzkirchen, Germany)

Docetaxel (Taxotere ${ }^{\circ}$; Sanofi Aventis, Bridgewater, NJ USA)

Ixebepilone (Ixempra®; Bristol-Myers Squibb, New York city, NY, USA)

Peloruside A, generously provided by Dr. Peter Northcote (Victoria University of Wellington, Wellington, NZ)

Glatiramer acetate (GA, Copaxone®) Teva Pharmaceutical Industries Ltd. (Petach Tikva, Israel) 
Risperidone was generously provided by Douglas Pharmaceuticals Limited, (Auckland, NZ)

MIS416 was generously provided by Innate Immunotheraputics (Auckland, NZ)

2.5 Compounds for in vitro use (if different from above)

Paclitaxel (LC laboratories, Woburn, MA, USA)

Docetaxel (LC laboratories, USA)

Laulimalide, generously provided by Dr. Peter Northcote (Wellington, NZ)

\subsection{In vivo techniques}

\subsubsection{Drug administration}

MSDs or their respective vehicles were diluted in dPBS (Invitrogen, Carlsbad, CA, USA) at indicated concentration to a final volume of $300 \mu \mathrm{l}$ and injected i.p. using a $27 \mathrm{~g}$ needle.

Risperidone was dissolved in $0.1 \mathrm{M}$ acetic acid to a stock concentration of $6 \mathrm{mg} / \mathrm{mL}$ then diluted to the required final concentration using mouse drinking water. To achieve correct daily dosages, average daily water consumption was measured before initiation of experiments and the correct dosage of risperidone was calculated accordingly.

Glatiramer acetate was diluted in dPBS (Invitrogen, USA) to a final volume of $100 \mu 1$ and injected s.c. into the scruff of the neck using a $29 \mathrm{~g}$ needle.

MIS416 or saline vehicle was injected undiluted at indicated amounts (between 100 and $250 \mu \mathrm{l}$ total volume) into the lateral tail vein using a 29 or $31 \mathrm{~g}$ needle.

Anti-interferon- $\gamma$ antibody (clone: AN18 (Prat et al., 1984)) was diluted in dPBS (Invitrogen, USA) to the indicated concentration in a final volume of $300 \mu 1$ and injected i.p. using a $27 \mathrm{~g}$ needle. 


\subsubsection{In vivo proliferation assay}

Cells from spleen and lymph nodes of 2D2 mice were isolated and processed into single cell suspensions. Following staining with $2 \mathrm{nM}$ carboxyfluorescein succinimidyl ester (CFSE; Sigma, USA), the reaction was quenched with FCS then cells were washed 3 times in dPBS (Invitrogen, USA) (see section 2.8.1). Following resuspension in dPBS (Invitrogen, USA), $2 \times 10^{7}$ 2D2 cells in a volume of $200 \mu 1$, were injected i.p. into B6.SJLptprca mice. One day later mice were immunised for EAE (see section 2.3.1). Mice were left untreated or were treated with $20 \mathrm{mg} / \mathrm{kg}$ paclitaxel i.p. on days $0-4$. Five days following immunisation, mice were culled and lymph nodes, spleen, blood and spinal cord were harvested and processed for flow cytometry analysis, as described (see sections 2.7 and 2.12).

\subsubsection{In vivo migration assay}

Cells from the spleen and lymph nodes of 2D2 mice were isolated and processed into single cell suspensions. Following staining with 2 nM CFSE (sigma, USA), the reaction was quenched with FCS then washed 3 times in dPBS (Invitrogen, USA). Following resuspension in dPBS (Invitrogen, USA), $2 \times 10^{7} 2 \mathrm{D} 2$ cells were injected i.p. into C57BL/6 mice. On the following day mice were either treated with $20 \mathrm{mg} / \mathrm{kg}$ paclitaxel i.p. or an equal volume of dPBS (vehicle) for 5 consecutive days. After the final injection $1 \mu \mathrm{g}$ of the chemokine CCL5 (RANTES; Peprotech Rocky Hill, NJ, USA) was injected in 100 $\mu \mathrm{l}$ dPBS (Invitrogen, USA) into the left hind flanks of the mice, while an equal volume of dPBS (vehicle) was injected into the right hind flank. Eighteen hours following hind flank injections, cells from the dLNs were isolated, counted and processed for flow cytometry analysis, as described (see sections 2.7 and 2.12). To control for potential variations in injection technique that could potentally affect cell numbers in the dLNs, additional paclitaxel (PTX) treated or untreated mice were injected with dPBS (Invitrogen, USA) in both the right and left hind flanks (i.e no CCL5 was administered to these mice). No differences in cell numbers between right and left LNs were observed in these mice (data not shown) 


\subsubsection{In vivo microtubule-stabilising drug combination treatments}

Peloruside stocks were dissolved in high-grade $100 \%$ ethanol. Clinical grade ixabepilone (Ixempra®; Bristol-Myers Squibb, USA) stocks were dissolved in the provided solvent (polyoxyethylated castor oil 52.8\% w/v and dehydrated ethanol 39.8\% w/v; Bristol-Myers Squibb, USA). Working concentrations were achieved by dilution of stocks in dPBS (Invitrogen, USA) to the indicated drug concentrations in a final injection volume of 300 $\mu 1$ per mouse. C57BL/6 mice were immunised for EAE (see section 2.3.1). Mice were injected i.p. on days 6, 8, 10 and 12 p.i. with indicated amounts and combinations of MSD drugs. Untreated animals were administered the equivalent concentration of ethanol and chemaphor vehicles that were present at the highest drug concentrations.

\subsubsection{Isolation of blood for serum cytokine analysis}

Mice were restrained, within a mouse restrainer and the distal tip of their tails nicked with sharp sterile scissors. Droplets of blood were collected in sterile microcentrifuge tubes then incubated at RT until the blood had coagulated. Following centrifugation $(12,000 \mathrm{~g}$ for 10 minutes) serum was removed and used immediately or stored at $-80^{\circ} \mathrm{C}$ until required.

\subsection{Ex vivo techniques}

\subsubsection{Dissection and organ removal}

Mice were sacrificed by $\mathrm{CO}_{2}$ asphyxiation and washed with absolute ethanol. The peritoneal cavity was opened and spleen and lymph nodes were removed using sterile tweezers. Bone and other organs were removed by blunt dissociation from connective tissue. Blood was harvested by cardiac puncture following cutting of the diaphragm and exposure of the heart. Progenitor cells for bone marrow derived macrophages were isolated from mice femurs via insertion of a $23 \mathrm{~g}$ needle into a cut end and flushing the marrow out with sterile dPBS (Invitrogen, USA). To perfuse, dPBS (Invitrogen, USA) was slowly injected into the left ventricle and blood was drained via the hepatic portal vein. Spinal cords were removed, following decapitation, by inserting a $19 \mathrm{~g}$ needle into the lumbar region of the vertebral column and exerting positive pressure onto the spinal cord via a syringe containing dPBS (Invitrogen, USA). 


\subsubsection{Preparation of a single cell suspension from lymphoid organs}

Spleens and lymph nodes were isolated and placed into wash buffer (see appendix 4). Single cell suspensions were made by dissociating tissue through $70 \mu \mathrm{m}$ cell strainers (BD Biosciences, Franklin Lakes, NJ, USA) and washing into $50 \mathrm{ml}$ centrifuge tubes. To remove red blood cells, splenocyte cell suspensions were incubated for 2 minutes in $2 \mathrm{mls}$ of red cell lysis buffer (Sigma, USA) before being washed in 8mls wash buffer, centrifuged at $700 \mathrm{~g}$ for 3 minutes and resuspended in fresh wash buffer. Live cell numbers were counted by trypan blue exclusion (see section 2.7.5).

\subsubsection{Preparation of a single cell suspension from Blood}

Mouse blood was harvested by cardiac puncture using a heparin (Sigma, USA) coated 27g needle on a $1 \mathrm{ml}$ syringe (BD Biosciences, USA). Blood samples were centrifuged at $1400 \mathrm{~g}$ for 5 minutes and the supernatant was removed. Red cells were removed by incubation in $2 \mathrm{mls}$ red cell lysis buffer (Sigma, USA) before being washed in $5 \mathrm{mls}$ wash buffer, centrifuged at $700 \mathrm{~g}$ for 3 minutes and resuspended. The above steps were repeated to ensure full lysis of red blood cells. Cells were resupended in FACS buffer before being stained for flow cytometry.

\subsubsection{Preparation of a single cell suspension from the spinal cord}

Single cell suspensions were prepared from spinal cords using a commonly used process (Sedgwick et al., 1991; Weir et al., 2006). Spinal cords were placed in a Petri dish and cut into small pieces using a scalpel blade. The tissue was then suspended in $5 \mathrm{ml}$ PBS containing $2.4 \mathrm{mg} / \mathrm{mL}$ type II collagenase (Invitrogen, USA) and incubated at $37^{\circ} \mathrm{C}$ for 30 minutes. To remove any clumps the tissue solution was repeatedly passed through a p1000 pipette tip and incubated for a further 10 minutes at $37^{\circ} \mathrm{C}$. Following passage through a 70 $\mu \mathrm{m}$ cell strainer (BD Biosciences, USA), tissue samples were centrifuged at $700 \mathrm{~g}$ for 3 minutes before the supernatant was discarded. Following resuspension in $10 \mathrm{ml} 37 \%$ Percoll (Sigma, USA) in Percoll dilutent (see appendix 4), cells were centrifuged at 700g 
for 30 minutes before the supernatant was removed. The cells were washed in FACs buffer before being stained for flow cytometry.

\subsubsection{Trypan Blue exclusion}

To count live cell numbers, cells were mixed with $0.4 \%$ Trypan blue (Sigma, USA) in a 1:1 or 10:1 dilution (in media) and counted on an improved Neubauer haemocytometer (Hawksley, Lancing, UK) using a compound microscope (CX41: Olympus, PA, USA).

\subsubsection{Histology}

Mice were perfused and spinal cords were isolated as described (see section 2.7.4), then fixed in Zinc fixative. Spinal cords were paraffin embedded, sectioned longitudinally, mounted on slide and stained with haematoxylin and eosin by staff at the Department of Pathology (University of Otago Wellington School of Medicine, NZ). Mounted sections were analysed using an Olympus IX51 inverted microscope (Olympus, PA, USA).

\subsection{In vitro techniques}

\subsubsection{CFSE staining}

Single cell suspensions (up to $1 \times 10^{8}$ ) were resuspended in $1 \mathrm{ml}$ dPBS (Invitrogen, USA) and mixed with $1 \mathrm{ml}$ of dPBS containing 1 or $2 \mu \mathrm{M}$ CFSE (Invitrogen, USA) and incubated in low light for 8 minutes at RT. To quench the reaction $2 \mathrm{mls}$ of FCS was added followed by $6 \mathrm{mls}$ of wash buffer. Following centrifugation (700g for 3 minutes) the sample was washed twice using $10 \mathrm{ml}$ of wash buffer. Cells were then resuspended in media or dPBS (Invitrogen, USA) depending on the specific application.

\subsubsection{Preparation of bone marrow derived macrophages}

Progenitor cells, flushed from mice femurs, were cultured $\left(5 \% \mathrm{CO}_{2}\right.$ at $\left.37^{\circ} \mathrm{C}\right)$ overnight in complete T cell medium (CTCM; see appendix 4) in 90mm petri dishes (Techno Plas, St Marys, Australia), before non-adherent cells were isolated and cultured in the presence of granulocyte-macrophage colony-stimulating factor and IL-3 (both $5 \mathrm{ng} / \mathrm{ml}$; Peprotech, 
USA). After 7 days adherent cells (macrophages) were detached by repetitive flushing with cold dPBS (Invitrogen, USA). For experiments, the bone marrow derived macrophages were plated at $1 \times 10^{5}$ cells/well in 96-well flat-bottomed plates (BD Biosciences, USA). Following overnight stimulation with $20 \mathrm{U} / \mathrm{ml}$ IFN- $\gamma$ (Sigma, USA), cells were incubated with or without lipopolysaccharide (LPS 200 ng/ml; Sigma, USA) and indicated drugs.

\subsubsection{In vitro cell proliferation assays}

Cells were resuspended in CTCM and cultured in duplicate or triplicate in U-bottomed 96well plates (BD Biosciences, USA) at a concentration of $1 \times 10^{6}$ or $5 \times 10^{5}$ cells per well for splenocytes and lymph node cells respectively. Cells were incubated $\left(5 \% \mathrm{CO}_{2}\right.$ at $\left.37^{\circ} \mathrm{C}\right)$ for 48 or $72 \mathrm{~h}$ in the presence or absence of concanavalin A (Con A; Sigma) or MOG ${ }^{35-55}$ respectively at indicated concentrations. If cytokines were to be measured, $100 \mu 1$ of supernatant was removed following indicated culture periods.

For measurement of proliferation by thymidine incorporation, cells were pulsed with 0.5 or $1 \mu \mathrm{Ci}\left[{ }^{3} \mathrm{H}\right]$ thymidine (GE Healthcare Little Chalfont, BU, UK) and cultured for an additional 8-16 hours before being frozen for later processing. Defrosted cells were harvested on glass fibre filters (PerkinElmer, Wellesley, MA, USA) using a cell harvester (Tomtec Inc., Hamden, CT. USA) and $\left[{ }^{3} \mathrm{H}\right]$ thymidine incorporation was measured as counts per minute $(\mathrm{CPM})$ using a $\beta$-counter (Microbeta trilux, PerkinElmer, USA). Cell proliferation is expressed as "proliferation index" (CPM of MOG or Con A wells/cpm of medium-alone wells).

For measurement of proliferation by CFSE dilution tissue was processed into a single cell suspension and stained with $1 \mathrm{mM}$ CFSE (Sigma, USA) as described above. For unstained controls the same procedure was carried out minus the addition of CFSE. Live cell numbers were counted by Trypan blue exclusion (see sections 2.7.5) and cultured in 96 well U-bottom plates (BD bioscience, USA). Following 48 or 72 hours in culture, supernatant was removed and cells were washed in FACs buffer before being stained for flow cytometry. 


\subsubsection{MTT assay}

Following incubation $\left(5 \% \mathrm{CO}_{2}\right.$ at $37^{\circ} \mathrm{C}$ ) of cells (see section 2.8.2) in flat bottom 96 well plates (BD Bioscience, USA) for a defined period of time (eg. 8 or 24 hours), $100 \mu 1 /$ well of supernatant was removed for cytokine analysis and $20 \mu \mathrm{l} /$ well 3-(4,5-dimethylthiazol-2yl)-2.5-diphenyrtetrazolium bromide) in dPBS (MTT solution, Sigma, USA) was added. After incubation for a further 2 hours, $100 \mu \mathrm{l} /$ well MTT solubliser was added and incubated at $37^{\circ} \mathrm{C}$ overnight. MTT reduction was measured by absorbance $(570 \mathrm{~nm}$ wave length) in a multiwell plate reader (Versamax, Molecular Devices, Sunnyvale, CA). Drug response was calculated as percent-control (\% control) (absorbance-blank/ average controlblank) x 100.

\subsubsection{In vitro synergy (using a CFSE dilution assay)}

Peloruside, Laulimalide, Docetaxel and Ixabepilone were reconstituted to $1 \mathrm{mM}$ in absolute ethanol and stored at $-80^{\circ} \mathrm{C}$. Working stocks $(800 \mathrm{nM}$ or $1600 \mathrm{nM})$ were diluted in media. Spleen cells were isolated and stained with CFSE as described (see section 2.7.2 and 2.8.1). Cells were cultured $\left(5 \times 10^{5}\right.$ cells/well) in 96-well round-bottom plates (BD Biosciences, USA). Following stimulation for 48 hours in the presence or absence of Con A ( $3 \mu \mathrm{g} / \mathrm{ml}$; Sigma, USA) and drug, the cells were harvested and stained for flow cytometry. Combination treatments and individual drug dose-response curves were conducted concurrently and in duplicate. Proliferation, represented as a reduction in CFSE fluorescence in cell progeny, was analysed using CellQuest Pro software (BD Biosciences, USA). A minimum of 10,000 events was collected for each sample, and results were calculated as the percentage of total splenocyte or $\mathrm{CD}^{+}$cell populations that had proliferated.

Drug interaction between MSD combinations were assessed using a combination index (CI) equation of Chou and Talalay and Berenbaum as follows: CI $=\left(D_{1} / D_{x 1}\right)+\left(D_{2} / D_{x 2}\right)$ (Berenbaum, 1985; Chou et al., 1984). A CI value equal to one indicates additive effects of two drugs, a value greater than one indicates a antagonistic relationship and a value less than one indicates a synergistic relationship. The doses $D_{1}$ and $D_{2}$ are the two drugs in combination and the $\mathrm{D}_{\mathrm{x} 1}$ and $\mathrm{D}_{\mathrm{x} 2}$ are the amounts of each drug alone that would produce the 
same response obtained with the drug in combination. To calculate the concentration of drug needed for a given response on its own the following logit-log equation was used: Dose $(x)+I C_{50} x$ (max-response/resonse-min $)^{1 / h i l l s l o p e}$ (Berenbaum, 1985). $\mathrm{IC}_{50}$, maximum, minimum and hillslope data was obtained using individual drug dose response curves analysed in Sigmaplot software. CI values were compared to the value of 1 using a onesample student's $t$-test.

2.9 Cytokine multiplex assays (CBA; Cytokine bead assay/Cytometric Bead Array)

CBA kits, either Mouse Th1/Th2 10plex FlowCytomix Multiplex (Bender MedSystems/ebioscience, San Diego, CA, USA) or Mouse Th1/Th2/Th17 Cytokine Kit (BD Bioscience, USA) were used. Protocols were conducted as per manufacture's instructions. Data was collected using either a FACScan or FACS Canto II flow cytometer (BD Biosciences, USA). Cytokine detection for the MIS416 experiments was conducted by Rebecca Girvan at Innate Immnotherapeutics (Auckland, NZ), using the same CBA kits as described above. The detection limit for individual cytokines ranged from $2-20 \mathrm{pg} / \mathrm{ml}$ as indicated in the manufactures instruction booklets.

\subsection{ELISA}

Enzyme linked immunosorbent assays (ELISA) were conducted as per manufacture's instructions (BD Bioscience, USA). 96 well ELISA Plates (BD Bioscience, USA) were coated overnight with a primary 'capture' antibody in sodium phosphate buffer at $4{ }^{\circ} \mathrm{C}$ (see appendix 4). The following day capture liquid was removed and plates were blocked using 5 or $10 \%$ FCS in PBS for 2 hours. After washing plates in ELISA wash buffer $(0.05 \%$ Tween 20 (Sigma, USA) in PBS), 50 $\mu$ l of standards and samples were added (if it was determined that samples needed dilution, samples were diluted in block solution). Following 2 hours of incubation, liquid from the samples/standards was removed and the plates were washed 4 times. Biotinylated secondary 'detection' antibody was added and incubated for 1 hour and then liquid was removed and the plate washed 6 times. Streptavidin conjugated horseradish peroxidase was then added to the plates and incubated for 1 hour. Following removal of any liquid, plates were washed 8 times. Tetramethyl 
benzidine (TMB) reagents $\mathrm{A}$ and $\mathrm{B}$ (BD Bioscience, USA) were mixed in equal volumes and $100 \mu \mathrm{l}$ was added to each well. After sufficient colour development the reaction was stopped by the addition of $100 \mu$ l of stop solution (0.18 M sulphuric acid). Absorbance values were measured at 450nM using a VersaMax (Molecular Devices, USA). A standard curve was constructed from the absorbance values of the standards and cytokine concentrations were calculated. The detection limits were $15-30 \mathrm{pg} / \mathrm{ml}$ for IL-12 and IFN $\gamma$ or $50-100 \mathrm{pg} / \mathrm{ml}$ for IL-10.

\subsection{Griess assay/reaction}

As nitric oxide (NO) is unstable and breaks down rapidly to nitrite and nitrate, measuring these breakdown products provides a relative measure of NO production (Sun et al., 2003). The Griess assay measures nitrite and is commonly used as an indicator of NO production in culture supernatant (Stuehr et al., 1985; Sun et al., 2003). Griess reagents A and B (Sigma, USA) were combined in equal volume and $50 \mu 1$ of this mixture was added to $50 \mu 1$ of culture supernatant in flat bottom 96 well plates (BD Bioscience). Serially diluted nitrite standards in duplicate were also included. Absorbance was measured at $570 \mathrm{nM}$ using a VersaMax (Molecular Devices, USA) and a standard curve of nitrite standards was produced from which unknown sample values were determined. The detection limit was 2$4 \mu \mathrm{M}$.

\subsection{General antibody staining and preparation of cells for flow cytometry}

Samples were washed in FACS buffer and Fc receptors blocked with 2.4G2 antibody (BD Biosciences, USA). Cells were stained with primary antibodies in a total volume of $50 \mu 1$ FACS buffer. All antibodies were pre-titrated, to optimise separation between cell populations and to minimise background fluorescence. Samples were incubated for 30 minutes at $4{ }^{\circ} \mathrm{C}$ or 15 minutes at RT. Excess buffer was added and samples were centrifuged at $600 \mathrm{~g}$ for 3 minutes and then resuspended. When biotin conjugated antibodies were used, cells were incubated for 10-20 minutes with strepavidin conjugated fluorophores, then washed with excess FACS buffer, centrifuged and resuspended. 
Samples were collected on a BD FACSScan or FACS Canto II using CellQuest pro or Diva software respectively (all BD Bioscience, USA). Data was analysed using Cellquest pro (BD Bioscience) or FLOJO 7 software (Tree Star, Ashland, OR, USA). See appendix 5 for a list of antibodies and strepavidin conjugated fluorophores used.

\subsection{Graphs and Statistics}

Graphs were produced using GraphPad Prism 5 (GraphPad Software Inc., La Jolla, CA, USA) or SigmaPlot 11 (Systat Software Inc, San Jose, CA, USA). Data analysis and statistical tests were conducted using GraphPad Prism 5, SigmaPlot 11 (Systat Software Inc, USA) or Microsoft Excel 2003 (Microsoft Corporation, Redmond, WA, USA). To test if values came from a Gaussian distribution a Shapiro-Wilk normality test was used. Correlation was assessed by the Pearson's test. The Student's t-test was used to determine statistical significance when comparing two treatment groups. In experiments for which more than two groups were compared a one-way ANOVA with a Newman-Keuls multiple comparisons test was used. If multiple treatment groups were being compared only to a control group, a one-way ANOVA with a Dunnett's test was used. For non-parametric tests comparing two groups a Mann-Whitney test was used. For non-parametric tests comparing more than two groups a Kruskal-Wallis test with Dunn's post-hoc analysis was used. Differences that gave $\mathrm{p}$ values $<0.05$ were considered statistically significant. 


\section{Chapter 3:}

Using microtubule-stabilising drugs to treat experimental autoimmune encephalomyelitis 


\subsection{Introduction}

Autoimmune diseases such as MS are mediated by the activation and proliferation of selfreactive $\mathrm{T}$ cells. Therefore one strategy to treat autoimmune disease is to use antiproliferative drugs to inhibit expansion of these cell populations. Mitoxantrone, an antimitotic drug, is already used for this purpose in the treatment of MS, and it is currently the only therapy licensed for progressive forms of the disease. However, due to irreversible cardiotoxicity, the use of mitoxantrone is very limited (Neuhaus et al., 2006). Microtubulestabilising drugs (MSDs) are another class of anti-mitotic compounds that have similar anti-proliferative effects but do not cause such severe and irreversible toxicity. Given the proven efficacy of inhibiting proliferation to treat MS, MSDs may provide a safe and effective treatment alternative. While there is a large body of research on the use of MSDs for the treatment of cancers, only a few studies have investigated these compounds within the context of autoimmune disease. This chapter explores the potential of MSDs to inhibit disease in an animal model of MS.

Microtubules are an important cytoskeletal component and have a diverse range of functions. As well as maintaining cellular structure, they are involved in intracellular transport, cell migration, polarisation and cell division (Hirokawa, 1998; Jordan et al., 2004; Nogales, 2001). Dynamic instability, the shortening and lengthening of the microtubule structures, is an important process in the normal functioning of microtubules (Erickson et al., 1992). Drugs that cause either stabilisation or destabilisation disrupt the dynamicity of the microtubule networks and ultimately cause perturbation of microtubule associated cellular process (Jordan et al., 2007). Microtubule destabilisers interfere with microtubule function by limiting the ability of the microtubule subunits ( $\alpha$ and $\beta$ tubulin) to polymerise into functional microtubules. Clinically microtubule destabilising drugs, such as vinorelbine and colchicine have been used for the treatment of cancers and inflammatory conditions, respectively (Aapro et al., 2011; Terkeltaub et al., 2010). In contrast to the destabilisers, MSDs stabilise microtubules and promote the polymerisation of tubulin subunits. Stabilisation of microtubules results in reduced microtubule dynamicity and can also cause microtubules to bundle together (Chan et al., 2011; Kamath 
et al., 2003). MSDs are used clinically in drug eluting stents to prevent restenosis, and for the treatment of various cancers (Altmann, 2001; Colmenarez et al., 2010).

As microtubules play a crucial role in mitosis the main cellular effect of MSDs is believed to be their anti-mitotic activity (Abal et al., 2003). In mitosis microtubules are important for the movement, alignment and subsequent separation of the chromosomes. Microtubules associated with this process are 10-100 fold more dynamic than cytoplasmic microtubules, which make them particularly sensitive to MSDs (Rusan et al., 2001; Zhai et al., 1996). Stabilisation of microtubules during mitosis results in cell cycle arrest and apoptosis (Jordan et al., 2007). The effectiveness of these compounds in inhibiting proliferation has made MSDs one of the most widely used class of cancer chemotherapeutics (Altmann, 2001; Jordan et al., 2004). While the mechanism of action of MSDs in cancer chemotherapy is inhibiting tumour cell proliferation, other advantageous effects such as inhibition of angiogenesis and cell motility also contribute (Belotti et al., 1996; Jordan et al., 2007).

Most MSDs are either isolated from natural products or are semi-synthetic derivatives. While there have been many MSDs identified, only a few have been developed for clinical use. The most commonly used MSD is the taxane, paclitaxel (PTX) which was originally isolated from the bark of the Pacific yew tree (Schiff et al., 1979). Due to limited solubility PTX is administered in Cremophor EL, a vehicle that consists of polyethoxylated castor oil and ethanol. However, Cremophor, itself is biologically active and causes both alterations in pharmacokinetics of co-administered drugs, as well as a hypersensitivity reaction in some people (Gelderblom et al., 2001; Gelderblom et al., 2002). An alternative taxane, also widely used, is docetaxel (DTX). DTX is a semi-synthetic analogue of PTX that does not need to be administered in Cremophor and has proved to be more effective in some forms of cancers (Crown et al., 2004). Ixabepilone (IXB), a semi-synthetic analogue of the compound epothilone $\mathrm{B}$, is the third MSD to be licensed as a cancer chemotherapeutic (Cobham et al., 2009). IXB has been shown to be less susceptible to active cellular drug efflux and is effective in treating some taxane-resistant cancers (Fojo et al., 2007; Rivera et al., 2011). 
The anti-proliferative activity of MSD, makes these drugs attractive compounds to explore in the context of autoimmune disease. Previous studies have found that PTX can inhibit collagen induced arthritis (a model of rheumatoid arthritis), and when administered after induction can suppress established disease (Brahn et al., 1994; Oliver et al., 1994). PTX has also been shown to be beneficial in treatment of immune mediated psoriasis (Ehrlich et al., 2004). Additionally, within EAE and spontaneous demyelination models of MS, PTX has been found to modify disease progression and severity in rats (Cao et al., 2000).

Research in our laboratory, using a mouse model of EAE, has found that both PTX or a novel MSD peloruside, show therapeutic potential in modifying disease expression in mice (Crume, 2007; Crume et al., 2009). When PTX or peloruside was administered on days 0-4 following EAE immunisation, onset of symptoms was delayed and incidence was reduced. This dosing regime however caused mortality between 7-12 days after immunisation in $30 \%$ of PTX treated mice. The toxicity only occurred in mice immunised for EAE and coincided with the time of disease onset in mice not treated with MSDs (7-14 days post immunisation) (Crume, 2007). Additionally this toxicity only occurred in PTX but not peloruside treated mice, suggesting that PTX may have a specific toxicity within the EAE model.

\subsection{Aims and Objectives}

This chapter further investigates the potential of MSDs for the treatment of EAE and explores the effectiveness of different treatment regimes. The current study also identifies if the toxicity seen in immunised drug-treated mice is specific to PTX or occurs following treatment with MSDs. As past research using MSDs in an autoimmune context has focused on PTX, the potential of other clinically available MSDs to treat EAE is determined in the present study. Finally, this study examines if the anti-proliferative actions of these drugs result in any alterations in chronic antigen-specific immune cell responses. 


\subsubsection{Specific Aims:}

1. To explore the potential of MSDs to modify EAE at different time points in disease process

2. To identify if toxicity observed in drug-treated mice is specific to paclitaxel

3. To assess if chronic antigen-specific immune responses are altered following MSD treatment 


\subsection{Results}

\subsubsection{Paclitaxel and docetaxel have similar disease modifying effects and toxicity}

Previous research conduced in our laboratory found that PTX administered on days 0-4 post immunisation (p.i.) delayed EAE onset. However, drug treatment caused mortality 712 days after immunisation in $30 \%$ of mice (Crume, 2007). This toxicity only occurred in immunised mice and coincided with the disease onset in immunised vehicle treated mice (d7-14 p.i.). A potential explanation for this mortality is the capacity of PTX to activate TLR-4 receptors and stimulate proinflammatory cytokine release (Byrd-Leifer et al., 2001). TLR-4 activation combined with the highly reactive inflammatory environment during EAE disease induction could result in an anaphylactic-like response.

To test if TLR activation by PTX was the cause of toxicity DTX was compared to PTX. Although DTX is a analogue of PTX it does not induce TLR-4 activation in mice (Fitzpatrick et al., 2003). Therefore, if toxicity was observed in the PTX but not DTX treated animals, this finding would support the possibility that the toxicity was associated with TLR-4 activation. As previous work had found that PTX is only effective at the maximum tolerated dose (MTD; 20mg/kg) (Crume et al., 2009), DTX was used at a dose near to the MTD $(10 \mathrm{mg} / \mathrm{kg})$. Mice that were immunised but untreated began to exhibit disease symptoms 16 days following immunisation with $80 \%$ disease incidence being reached 30 days p.i. (Figure 3.1a and b). Both PTX and DTX treated mice had delayed disease onset until day 30, which is consistent with previous research using PTX (Crume, 2007). Despite the delayed onset of disease in drug treated animals, once disease symptoms had established no reduction in incidence or difference in chronic disease courses was observed in ether PTX or DTX treated mice compared to untreated (Figure

3.1a and b). Although no symptoms of EAE were evident, toxicity was observed on days 6 to 9 post immunisation with 20\% of PTX and DTX-treated mice dying during this period. Some of the mice that exhibited toxicity also displayed symptoms of dyspnea, a hunched posture and reduced activity when compared to other mice within the drug treated groups. Similar symptoms however can also sometimes be observed in untreated mice just prior to symptom onset. 
Collectively these results support the previous findings that while PTX does delay disease onset it also causes toxicity in 20-30\% of the drug treated mice (Cao et al., 2000; Crume, 2007). As fatalities also occurred in DTX treated animals, it is unlikely TLR-4 activation is associated with the observed drug toxicity. Furthermore, the finding that toxicity occued in both PTX- and DTX-treated animals indicates that the observed effect is not related to the PTX vehicle cremophor, as DTX is administered in polysorbate 80, instead of cremophor.
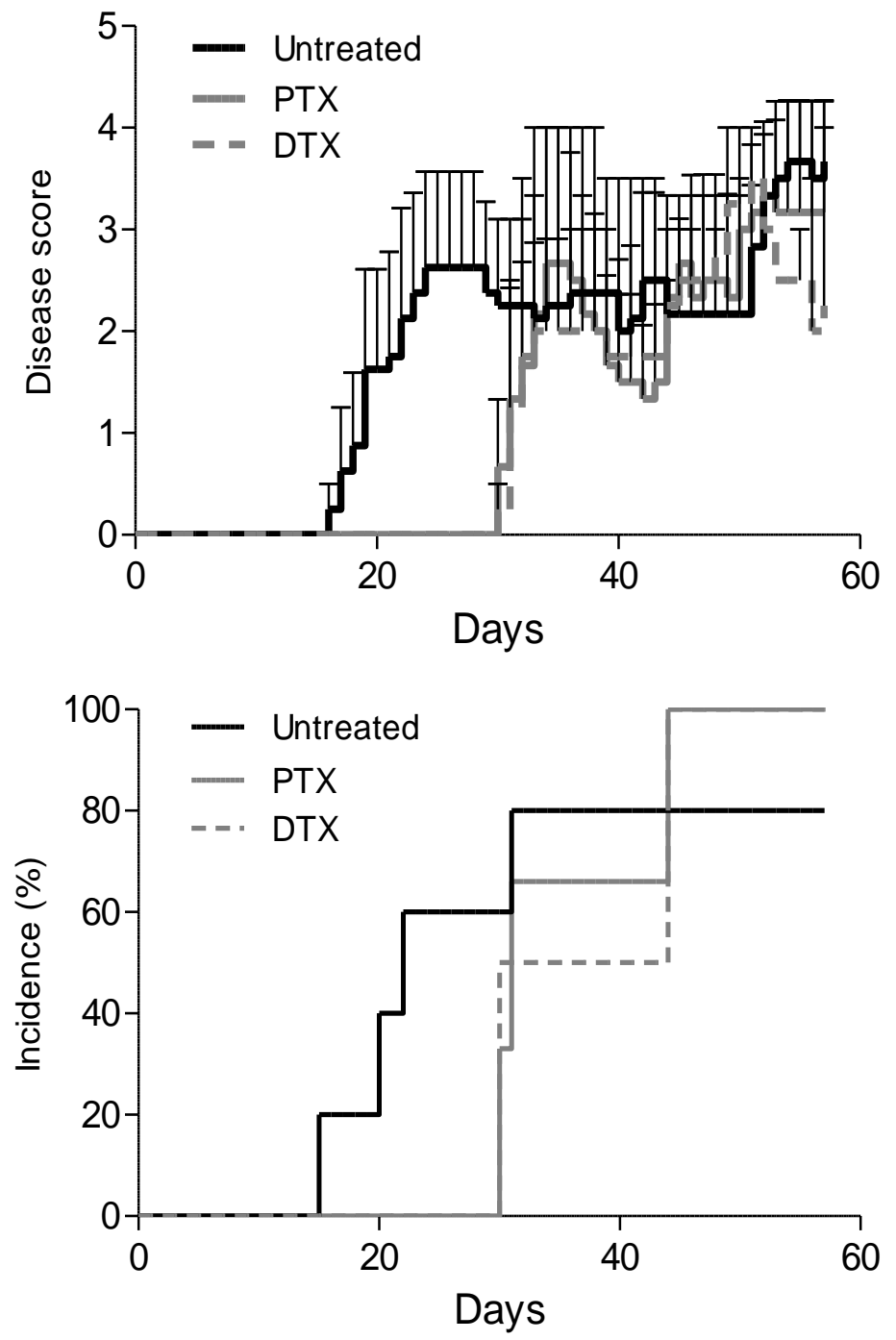

Figure 3.1 Paclitaxel or docetaxel treatment (d0-4 p.i.) delays EAE disease onset C57BL/6 mice were immunised for EAE and treated with paclitaxel (PTX; $20 \mathrm{mg} / \mathrm{kg}$ ) or docetaxel (DTX; $10 \mathrm{mg} / \mathrm{kg}$ ) daily on days 0-4 p.i. or left untreated. (a) Disease score of sick mice (b) Disease incidence; calculated as percent of mice with clinical disease ( $\geq 0.5$ on disease score). Data represents one of two identical experiments. $(n=3-5$ mice per group). Any mice that died were excluded from the analysis. 


\subsubsection{Paclitaxel and docetaxel treatment does not inhibit the development of antigen- specific responses in peripheral $T$ cells}

Since the main effect of MSDs is considered to be anti-mitotic, it is possible that the alteration in disease expression in PTX and DTX treated mice was due to inhibition of immunisation-induced $\mathrm{T}$ cell proliferation. If administration of MSDs was inhibiting clonal expansion of antigen-specific $\mathrm{T}$ cells, it is likely that antigen-specific responses in the peripheral lymphoid organs of MSD treated mice would be reduced. As MS is a chronic disease any new treatment would ideally lead to long-term alterations in encephalitogenic immune responses. In the context of EAE, chronic changes of antigen-specific $\mathrm{T}$ cell responses could provide an indication of long-term alterations in encephalitogenic immune responses. Thus initial investigations into the effects of MSDs on the immune system focused on antigen-specific $\mathrm{T}$ cell responses present in the chronic stages of disease (i.e. $\geq$ 30 days p.i.).

To determine if PTX or DTX treatment chronically affected peripheral T cell proliferative responses, splenocytes were isolated 30 or 57 days after immunisation and cultured individually in the presence of a polyclonal $\mathrm{T}$ cell stimulus, concanavalin $\mathrm{A}$ (Con $\mathrm{A}$ ) or myelin oligodendrocyte glycoprotein peptide 35-55 (MOG). No significant difference in splenocyte proliferation occurred in response to Con A stimulation (Figure 3.2a). In contrast antigen-specific $\mathrm{T}$ cell responses to MOG were higher in splenocytes from either PTX or DTX treated mice when compared to untreated mice (Figure 3.2b). This result may indicate that during the cronic stages of disease more antigen-specific $\mathrm{T}$ cells are still present within the spleen of PTX or DTX mice compared to untreated mice. The observed changes in proliferation were not due to alterations in total percentages of $\mathrm{T}$ cells within the spleens between any of the treatment groups (Figure 3.3a-c). Proliferation in response to MOG indicates the presence of an EAE specific T cell response; while, proliferation in response to Con A indicates total $\mathrm{T}$ cell responses. Therefore, these results indicate that PTX and DTX does not inhibit the development of long term antigen-specific $\mathrm{T}$ cell responses following immunisation nor do they alter the total $\mathrm{T}$ cell population's proliferative capacity. 
IFN- $\gamma$ is a classic indicator of a Th1 type inflammatory response and can contribute both to pathogenesis and protection in EAE (Sabatino et al., 2008; Williams et al., 2011; Yura et al., 2001; Zhang, 2007). To investigate if the drugs also resulted in altered IFN- $\gamma$ production, supernatants from the splenocyte cultures were analysed. Cells from PTX treated mice produced significantly more IFN- $\gamma$ at the highest concentration of Con A compared to DTX and untreated groups (Figure 3.4a). Cells from PTX treated mice also produced increased IFN- $\gamma$ in response to MOG but this increase was only apparent at the low concentration of $3 \mu \mathrm{g} / \mathrm{ml}$ MOG (Figure 3.4b). This increase in IFN- $\gamma$, however, does not correlate to either disease protection or exacerbation, as there was no substantial diffrence between disease scores of drug treated and untreated mice (Figure 3.1 and data not shown). Taken together these results suggest that PTX and DTX treatment does not prevent the induction of antigen-specific responses in this model.
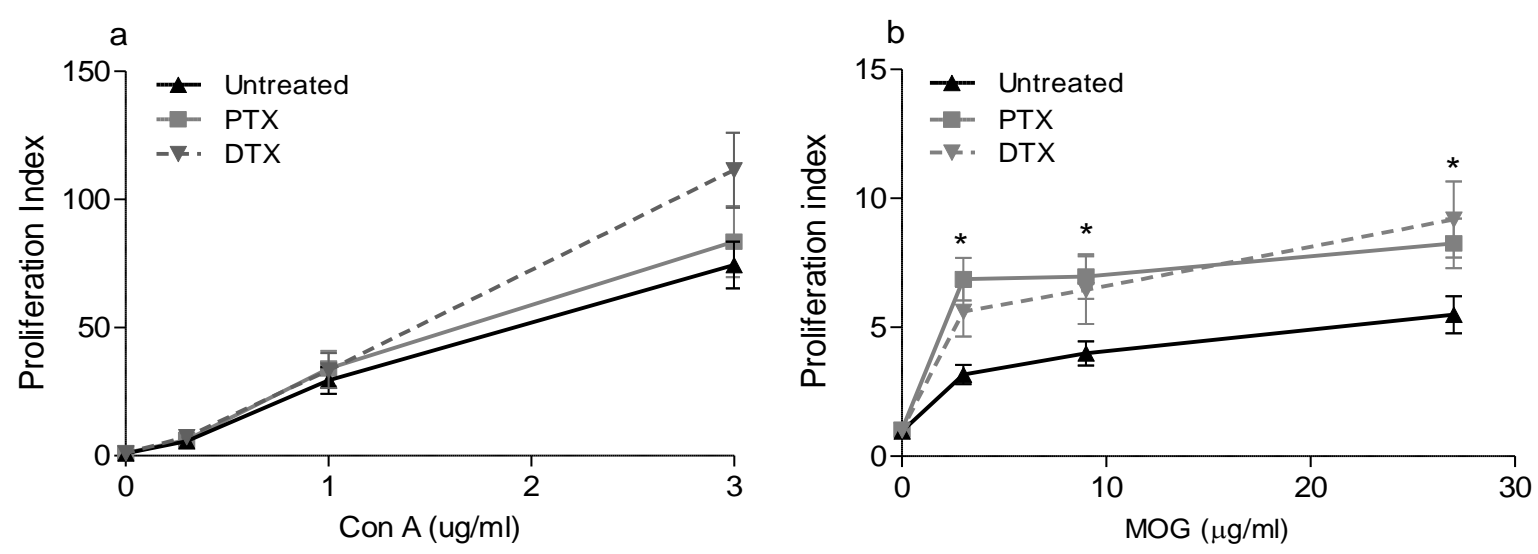

Figure 3.2 Paclitaxel or docetaxel treatment (d0-4 p.i.) does not inhibit ex vivo antigen-specific proliferation

C57BL/6 mice were immunised for EAE and treated with paclitaxel (PTX; $20 \mathrm{mg} / \mathrm{kg}$ ) or docetaxel (DTX; $10 \mathrm{mg} / \mathrm{kg}$ ) daily on days 0-4 p.i. or left untreated. Spleen cells were cultured in medium alone or in the presence of (a) Con A or (b) MOG, 30 or 57 days p.i. Proliferation was assessed by ${ }^{3}[\mathrm{H}]$ thymidine $(1 \mu \mathrm{Ci})$ incorporation and is expressed as Proliferation index; CPM of stimulated wells/CPM of media alone. Shown are means and SEM of individual mice from the two experiments ( $\mathrm{n}=8-9$ mice/group). $* \mathrm{P}<0.05$ significant differences between PTX and DTX compared to untreated, within each of the stimulation concentrations by one-way ANOVA with Newman-Keuls Multiple Comparison test. 

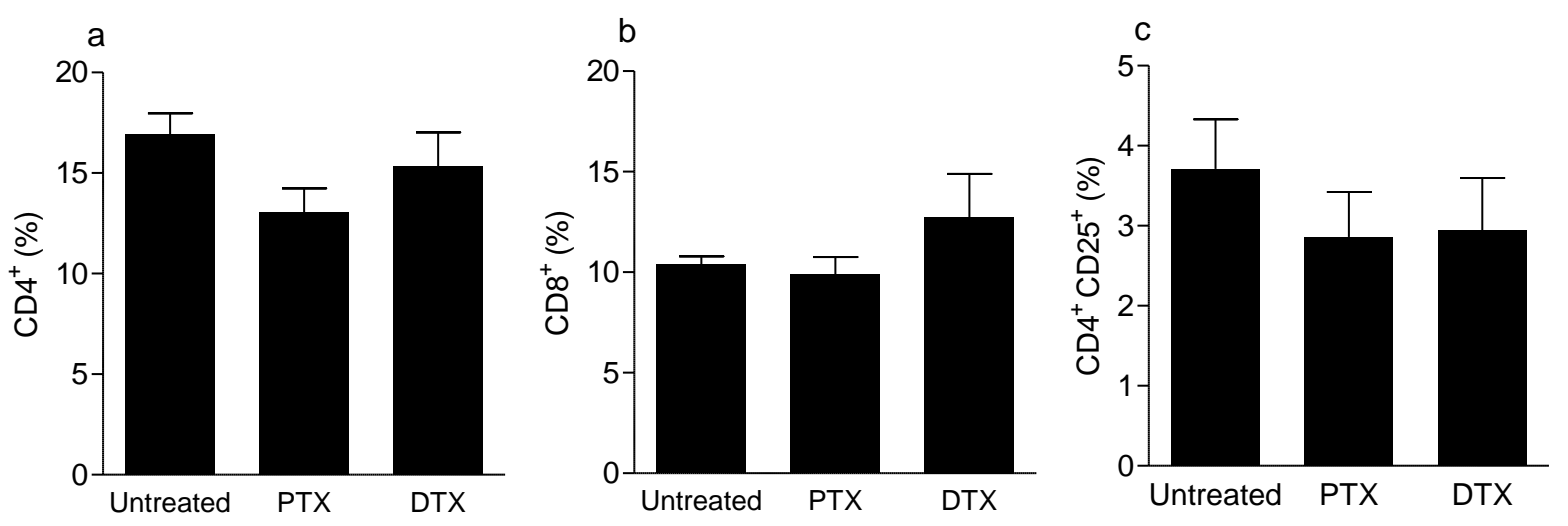

Figure 3.3 Paclitaxel or docetaxel treatment (d0-4 p.i.) does not alter the percentage of $T$ cells in the spleen

C57BL/6 mice were immunised for EAE and treated with paclitaxel (PTX; $20 \mathrm{mg} / \mathrm{kg}$ ) or docetaxel (DTX; $10 \mathrm{mg} / \mathrm{kg}$ ) daily on days $0-4$ p.i. or left untreated. Splenocytes were isolated 30 or 57 days p.i. and analysed by flow cytometry for percentages of total (a) $\mathrm{CD} 4^{+}$, (b) $\mathrm{CD} 8^{+}$and (C) $\mathrm{CD} 4^{+} \mathrm{CD} 25^{+}$cells. Shown are means and SEM of individual mice from the two experiments $(n=8-9$ mice/group). There were no significant differences between treatment groups by one-way ANOVA with Newman-Keuls Multiple Comparison test.
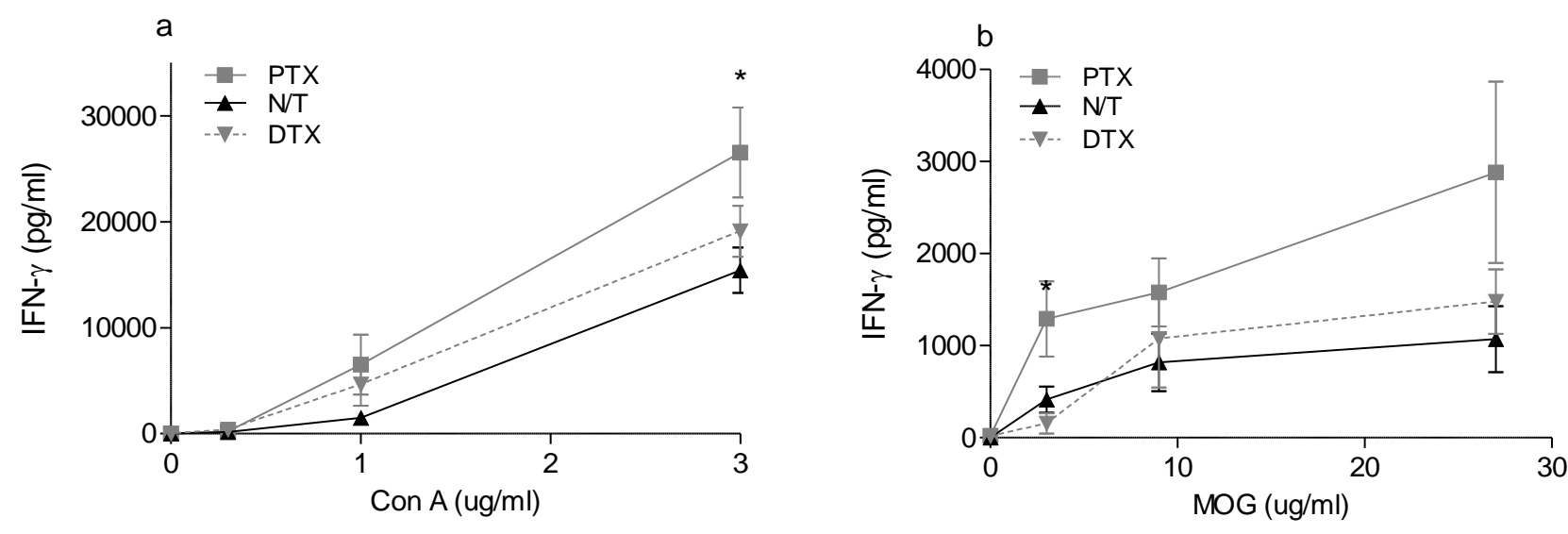

Figure 3.4 Paclitaxel or docetaxel treatment (d0-4 p.i.) does not inhibit ex vivo IFN- $\gamma$ production C57BL/6 mice were immunised for EAE and treated with paclitaxel (PTX; $20 \mathrm{mg} / \mathrm{kg}$ ) or docetaxel (DTX; $10 \mathrm{mg} / \mathrm{kg}$ ) daily on days 0-4 p.i. or left untreated (N/T). Spleen cells were cultured in medium alone or in the presence of (a) Con A or (b) MOG for 48 and 72 hours respectively. At 30 or 57 days p.i.. IFN- $\gamma$ production was assessed by ELISA in culture supernatants. Shown are means and SEM of individual mice from the two experiments ( $\mathrm{n}=8-9$ mice/group). ${ }^{*} \mathrm{P}<0.05$ significant difference between PTX compared to DTX and untreated by one-way ANOVA with Newman-Keuls Multiple Comparison test. 


\subsubsection{Treating after the initial inflammatory response delays disease onset}

Administration of PTX or DTX immediately following immunisation may inhibit the induction of an encephalgenic immune response rather than modifying the inflammatory milieu. In order for a treatment to be of therapeutic benefit within the context of MS, it would have to be effective after an already established encephalgenic immune response. Previous research has shown that in EAE the induction and proliferation of encephalgenic cells occurs within the first week following immunisation (Hofstetter et al., 2010; Targoni et al., 2001). To explore if treating after this period with PTX or DTX modifies disease, mice were treated on d6-10 p.i. Similar to the d0-4 p.i. treatment, MTDs of PTX or DTX significantly delayed disease onset and reduced disease incidence at d30 p.i. (Figure 3.5a and b) Interestingly, a similar rate of toxicity was observed between d7-11 p.i. as seen in the d0-4 p.i. treatment. Again this period of toxicity appeared in a similar time frame to when immunised untreated mice began to exhibit EAE symptoms. This suggests that toxicity coincides with CNS immune infiltration.

Thirty days following immunisation, spleens and dLN were analysed for potential changes in antigen-specific responses or cellularity. No difference in $\mathrm{CD} 4^{+}, \mathrm{CD}^{+}, \mathrm{CD} 4^{+} \mathrm{CD} 25^{+} \mathrm{T}$ cells or total cell numbers, within either the spleens or dLN was observed between treatment groups (Note: only 2-4 mice per drug treatment group) (Figure 3.6a-d). To investigate if antigen-specific responses were altered by drug treatment, splenocytes and LN cells, from individual animals were cultured in the presence of Con A or MOG. Only LN cells from DTX treated mice exhibited an increased proliferative response following Con A stimulation relative to the untreated group; while, no difference was not observed in splenocytes (Figure 3.7a). Cells from PTX and DTX treated mice exhibited no difference in proliferative response to MOG when compared to untreated immunised mice in either splenocyte or LN cultures (Figure 3.7b). Additionally no difference in levels of IFN- $\gamma$ was detected between splenocytes or LN cells from any of the treatment groups when stimulated with either Con A or MOG (Note: only 2-4 mice per drug treatment group) (Figure 3.8a and b). 
These results indicate that despite altering disease progression PTX and DTX, administered on d6-10 p.i., do not cause permanent alterations in antigen-specific $\mathrm{T}$ cell proliferative responses. Additionally, no difference in IFN- $\gamma$ production or total $\mathrm{T}$ cell numbers was observed suggesting that the development of $\mathrm{T}$ cell responses occurs similarly in drug-treated and untreated, immunised mice. This finding suggests that any effect these drugs have on the immune system are transient and only occur either during or directly after drug treatment. Alternatively, PTX or DTX treatment may not be causing any direct effect on the encephalgenic immune response, and the alteration in disease progression in drug-treated animals occurs due to non-immune associated factors.
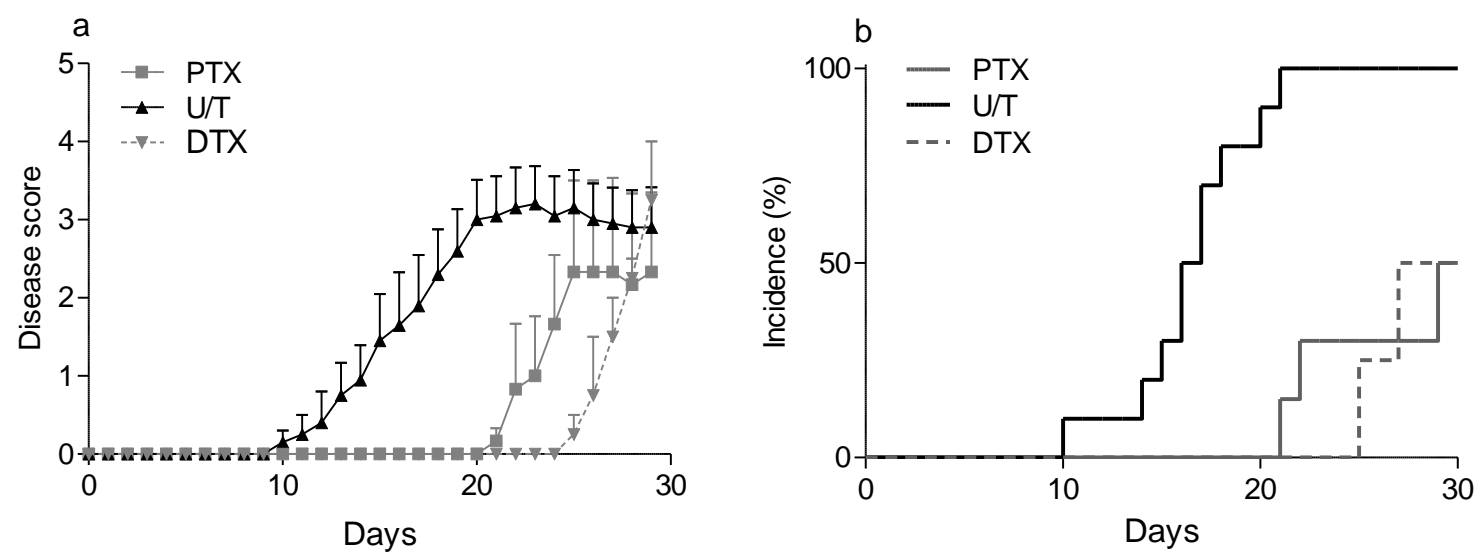

Figure 3.5 Paclitaxel or docetaxel treatment (d6-10 p.i.) delays EAE disease onset

C57BL/6 mice were immunised for EAE and treated with PTX $(20 \mathrm{mg} / \mathrm{kg})$ or DTX $(10 \mathrm{mg} / \mathrm{kg})$ daily 6-10 p.i. or left untreated. (a) Disease score of sick mice (b) Incidence = cumulative disease incidence; calculated as percent of mice with clinical disease.. Data represents two experiments (n $=4$ PTX, 4 DTX, 10 untreated). Any mice that died were excluded from the analysis. 

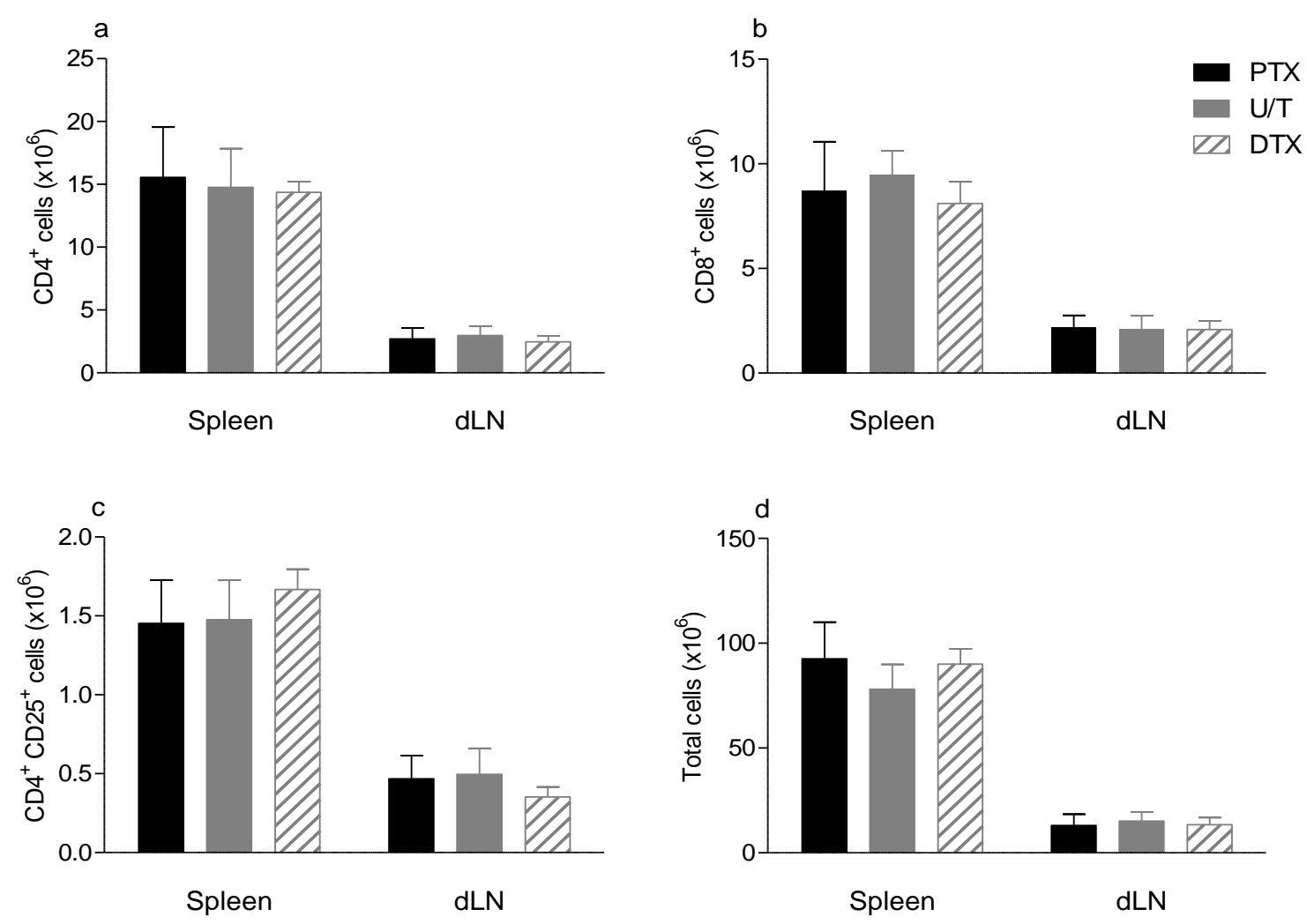

Figure 3.6 Paclitaxel or docetaxel treatment (d6-10 p.i.) does not chronically alter spleen or lymph node cellularity

C57BL/6 mice were immunised for EAE and treated with PTX $(20 \mathrm{mg} / \mathrm{kg})$ or DTX $(10 \mathrm{mg} / \mathrm{kg})$ daily 6-10 p.i. or left untreated. Splenocytes and lymphocytes were isolated 30 days p.i. counted and analysed by flow cytometry to calculate total (a) $\mathrm{CD}^{+}$, (b) $\mathrm{CD} 8^{+}$, (c) $\mathrm{CD} 4^{+} \mathrm{CD} 25^{+}$ and (d) total cell numbers. Shown are means and SEM of individual mice from one experiment ( $\mathrm{n}=2$ PTX, 4 DTX, 5 untreated). There were no significant differences between treatment groups within spleen or dLN analysed by one-way ANOVA with Newman-Keuls Multiple Comparison test
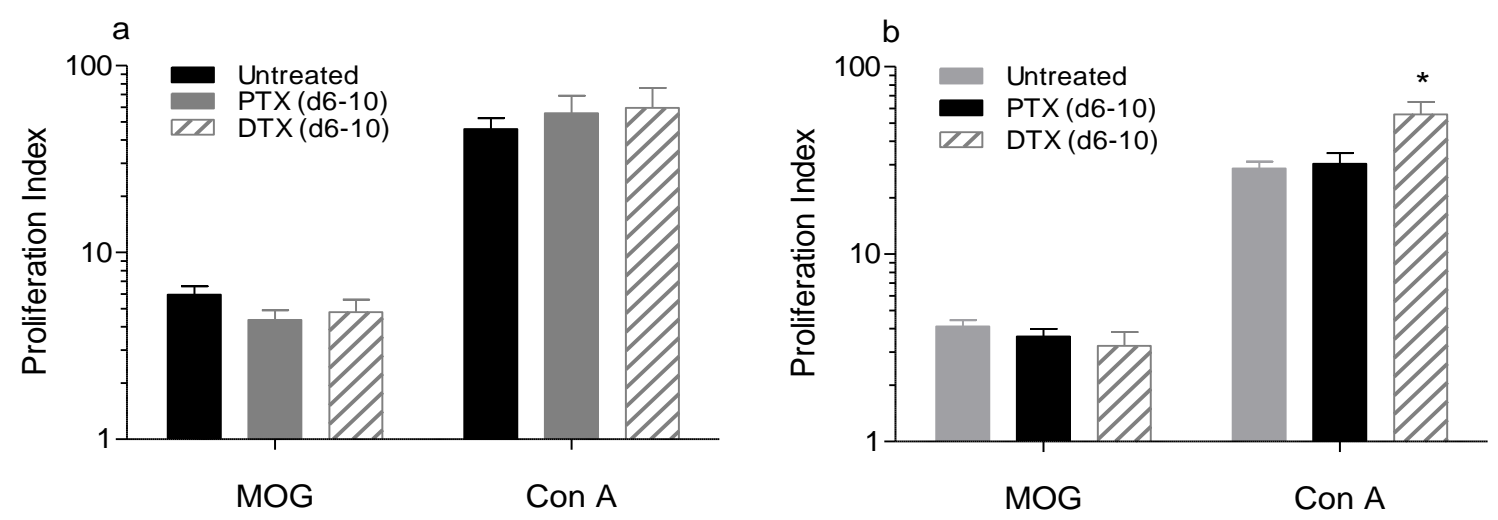

Figure 3.7 Paclitaxel or docetaxel treatment (d6-10 p.i.) does not inhibit ex vivo antigenspecific proliferation

Spleen (a) or dLN (b) cells were cultured in medium alone or in the presence of MOG $(27 \mu \mathrm{g} / \mathrm{ml})$ or Con $(3 \mu \mathrm{g} / \mathrm{ml}), 30$ days p.i.. Proliferation was assessed by ${ }^{3}[\mathrm{H}]$ thymidine $(1 \mu \mathrm{Ci})$ incorporation and is expressed as Proliferation index; CPM of stimulated wells/CPM of media alone. Shown are means and SEM of individual mice from two experiments ( $\mathrm{n}=6$ PTX, 4 DTX, 10 untreated). ${ }^{*} \mathrm{P}<$ 0.05 significant difference between groups within each of the stimulation conditions by one-way ANOVA with Newman-Keuls Multiple Comparison test. 

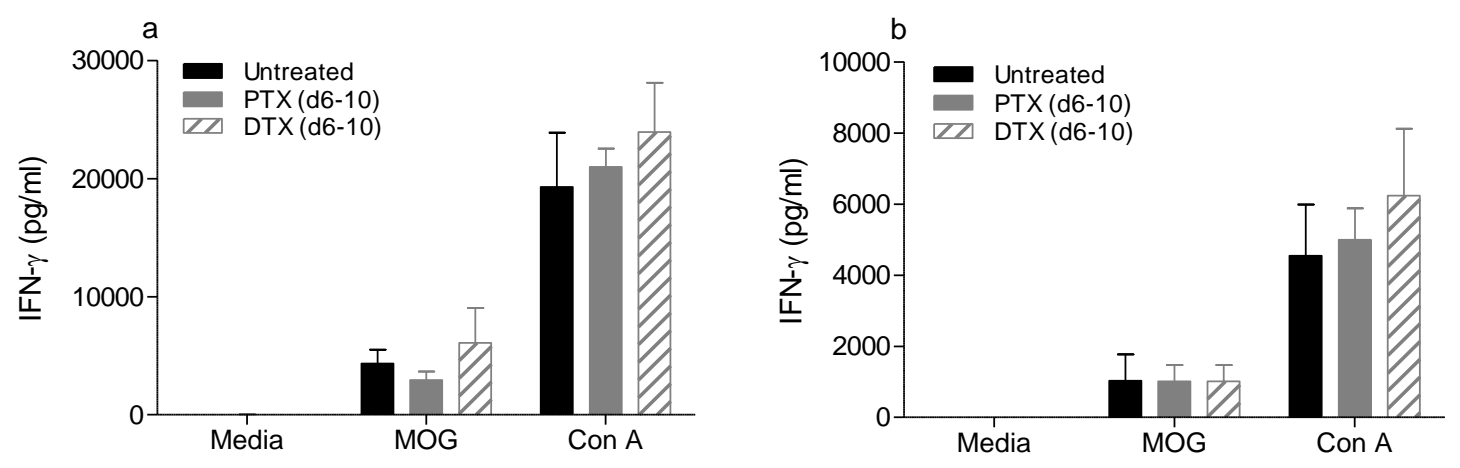

Figure 3.8 Paclitaxel or docetaxel treatment (d6-10 p.i.) does not inhibit ex vivo IFN- $\gamma$ production

Spleen (a) or dLN (b) cells were cultured in medium alone or in the presence of Con A ( $3 \mu \mathrm{g} / \mathrm{ml})$ or MOG $(27 \mu \mathrm{g} / \mathrm{ml})$ for 48 and 72 hours respectively. At 30 days p.i. IFN- $\gamma$ production was assessed by ELISA in culture supernatants. Shown are means and SEM of individual mice from one experiment ( $\mathrm{n}=2$ PTX, 4 DTX, 5 untreated). There were no significant differences between groups within each of the stimulation conditions by one-way ANOVA.

\subsubsection{A dual dosing regime delays disease but does not reduce long-term disease burden}

To explore if repeating the drug treatment could provide a prolonged therapeutic benefit, immunised mice were injected with PTX or DTX on days 6-10 p.i. and then again on days 21-25 p.i. Interestingly no deaths were seen after PTX or DTX treatment at d6-10. One mouse from the DTX treatment group died on d29 p.i. after exhibiting abnormal disease symptoms that included loss of balance and hind limb tone, but normal tail movement, which is unexpected when hindlimb weakness is occuring. The mouse exhibited no symptoms that were consistent with those seen in the presence of drug toxicity, making it ambiguous as to wheather the drug treatment was the cause of this death. However, due to the timing of the death it is likely that drug treatment was a contributing factor.

While a substantial delay in full disease severity occurred in all drug treated groups, little extra benefit in overall disease burden was achieved with the administration of a second PTX or DTX treatment on d21-25 p.i. (Figure 3.9a). Despite this, an increased delay in disease onset occurred in both d21-25 p.i. treatment groups (Figure 3.9b). This was particularly pronounced following DTX treatment with only 40\% incidence until around day 40. As a treatment group receiving a single dosing regime of DTX was not included in this experiment, the possibility that this delay could have occurred following a single dosing regime cannot be excluded. These results indicate that using a dual dosing regime 
of treatment at d6-12 and d21-25 confers little benefit to altering long-term disease severity or disease burden but may further delay disease onset.
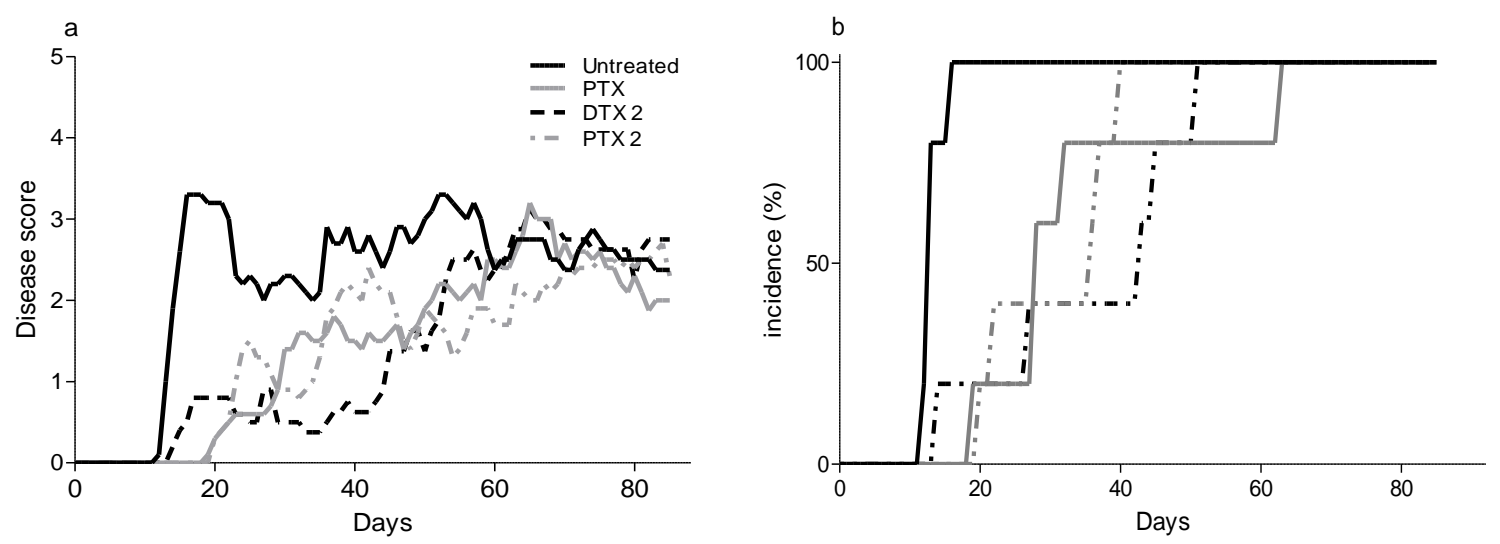

Figure 3.9 A dual dosing regimen of paclitaxel or docetaxel does not improve in vivo efficacy C57BL/6 mice were immunised for EAE and treated with paclitaxel $(20 \mathrm{mg} / \mathrm{kg})$ or docetaxel $(10 \mathrm{mg} / \mathrm{kg})$ daily on days 6-10 p.i. or left untreated. An additional 5 day course of paclitaxel (PTX 2) or docetaxel (DTX 2) was administered following disease onset (d21). a) Disease score of all mice (b) Disease incidence; calculated as percent of mice that develop clinical disease. Data represents one experiment $(\mathrm{n}=$ 4-5 mice per group). Any mice that died were excluded from the analysis.

\subsubsection{Ixbepilone alters disease expression without causing toxicity}

Ixabepilone (IXB) is a MSD that is structurally unrelated to the taxanes. To test if IXB, like PTX could alter disease expression in EAE a comparison of the two MSD was conducted. Previous studies in mouse cancer models found IXB to be well tolerated when administered at $10 \mathrm{mg} / \mathrm{kg}$ every other day (QOD) over a 7 day period. Using this treatment strategy, mice were immunised and treated on day 6-12 p.i. QOD with IXB. Because previous experiments had shown a daily PTX treatment at 6-10 p.i. effective, this strategy was compared to PTX administered on d6-12 p.i. QOD. Mice receiving PTX d6-12 p.i. QOD or daily doses on d6-10 p.i. exhibited a similar disease onset and disease severity (Figure 3.10a and b). While one mouse died (d13 p.i.) following PTX d6-10 p.i. treatment and all mice within this group exhibited significant weight loss (Figure 3.10c), no toxicity or weight loss was observed in the IXB or PTX QOD d6-12 p.i. treated animals. This observation may indicate that the toxic effects of the drug are associated with exposure to prolonged high drug concentrations. The QOD treatment would result in lower peak drug concentrations as more drug would be excreted or metabolised between doses. Additionally QOD treatment on d6-12 p.i. involves administration of a total of 4 doses 
compared to 5 doses in the daily treatment. The extra dose in the daily treatment may exacerbate the toxic effects but is unlikely to be the sole cause of toxicity, as weight loss in the PTX daily treatment group is already apparent as early as d8-9 p.i. This loss of weight is prior to the administration of the final dose on $\mathrm{d} 10$ p.i. indicating that toxicity may begin to occur part way through the daily treatment regime.

Only 1 out of 5 mice treated with IXB exhibited disease symptoms and these resolved within ten days of onset (Figure 3.10a). This contrasts with PTX treated mice of which $60 \%$ exhibited disease by d30 p.i. with no sign of disease resolution. However, in subsequent experiments, IXB treatment did not cause such drastic alterations in disease. The IXB (d6-12 p.i. QOD) treated mice had a similar delay of disease onset compared to the PTX (d6-12 p.i. QOD) treated mice and disease severity and long-term disease burden were similar (Figure 3.11a and b). The delay in disease onset within drug treated mice was also not as pronounced as was seen in previous experiments (eg. Figures 3.1 and 3.5). When experiments using PTX and IXB d6-12 p.i. QOD are collectively compared, a similar delay in disease onset and reduction in disease burden can be observed for both drugs (Table 3.2). However IXB treatment also led to a significant reduction in peak disease score (untreated $=3.9+/-0.1$ vs. $\mathrm{IXB}=3.3+/-0.3$ ) and the maximal disease score reached for any mouse was 4 compared to 4.5 and 5 for PTX and untreated mice respectively. These results suggest that IXB may provide a small therapeutic advantage over PTX.

Spleen and dLN were analysed for changes in cellularity 30 days following immunisation. No difference in $\mathrm{CD}^{+}, \mathrm{CD}^{+} \mathrm{T}$ cell numbers or total cell numbers in the spleen or dLN was observed between any of the treatment groups (Figure 3.12a-d). Additionally, no significant difference in proliferative response of splenocytes and LN cells was observed between groups, when cultured in the presence of Con A or MOG (Figure 3.13a and b). These results indicate that IXB like PTX does not appear to alter long-term peripheral T cell responses. 

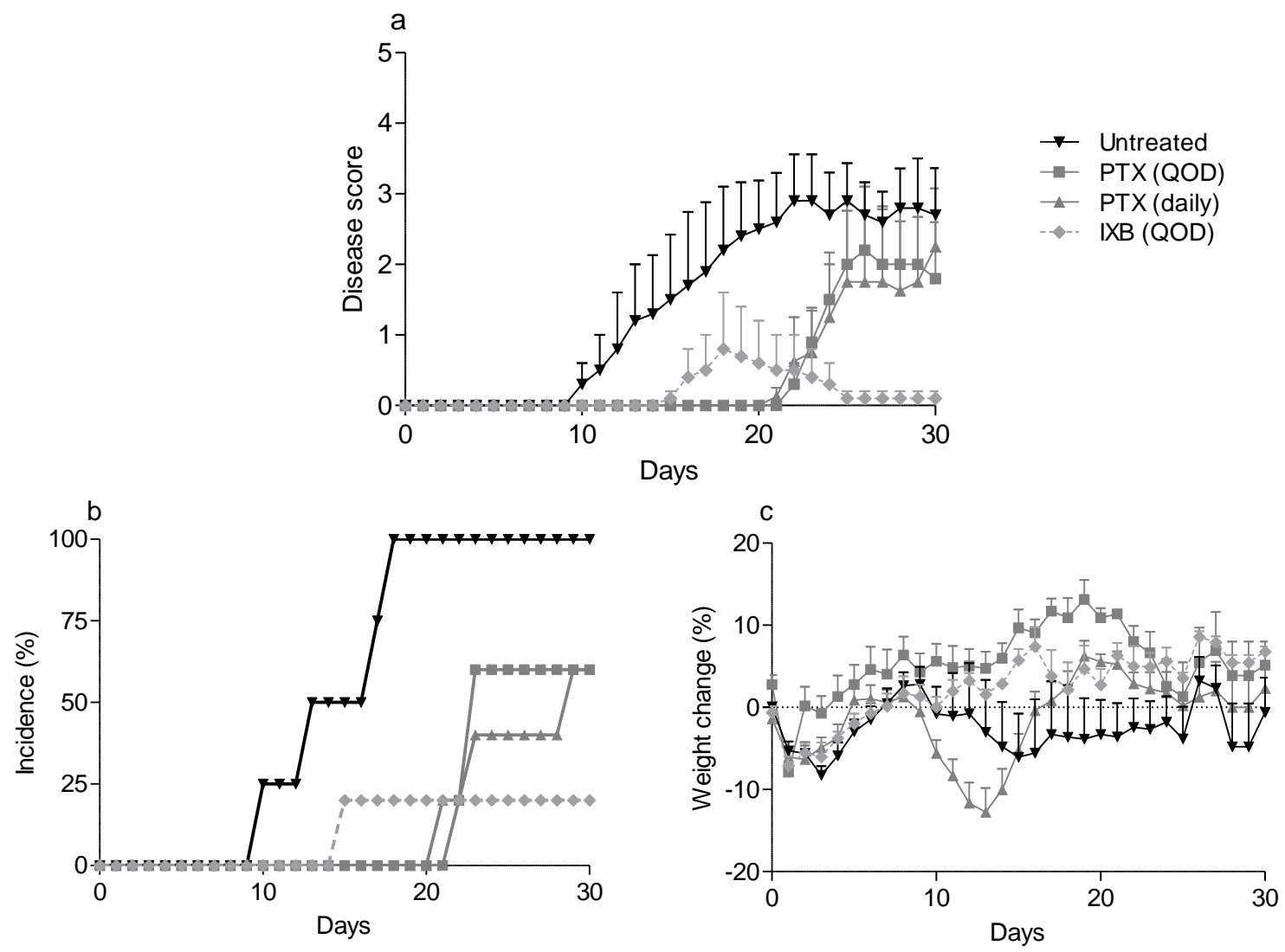

Figure 3.10 Ixabepilone treatment (d6-12 p.i.) delays EAE onset and severity

C57BL/6 mice were immunised for EAE and treated with paclitaxel $(20 \mathrm{mg} / \mathrm{kg})$ on days $6-10$ or days 6-12 QOD or with Ixabepilone (10mg/kg) on days 6-12 QOD. a) Disease score of all mice (b) Disease incidence; calculated as percent of mice that develop clinical disease. (c) \% weight change; calculated as $\%$ change of individual mice from d0. Data represents one experiment $(n=4-5$ mice per group). Any mice that died were excluded from the analysis.
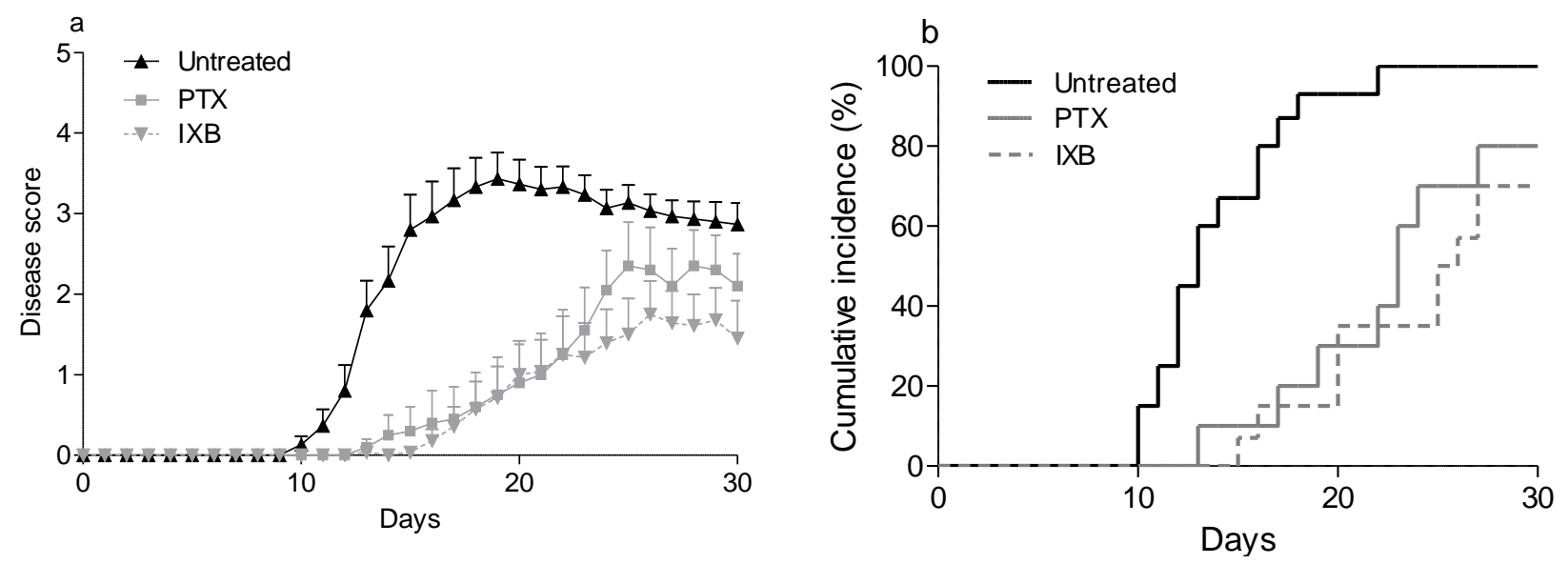

Figure 3.11 Ixabepilone treatment (d6-12 p.i.) exhibits similar efficacy to paclitaxel in delaying EAE onset

C57BL/6 mice were immunised for EAE and treated with paclitaxel $(20 \mathrm{mg} / \mathrm{kg})$ or with Ixabepilone $(10 \mathrm{mg} / \mathrm{kg}$ ) on days 6-12 QOD. a) Disease score of all mice (b) Disease incidence; calculated as percent of mice that develop clinical disease. Data represents three experiments ( $n=10$ PTX, 14 IXB and 15 untreated). 
Table 3.1: Paclitaxel and docetaxel alter EAE disease parameters

\begin{tabular}{|l|l|l|l|}
\hline Drug Treatment & Untreated & Paclitaxel & Docetaxel \\
\hline Incidence & $80 \%(25)$ & $65 \%(19)$ & $42 \%(19)$ \\
\hline Mortality before disease onset & $0 \%$ & $25 \%(5)$ & $20 \%(4)$ \\
\hline Day of onset & $13.8 \pm 1.4$ & $22.3 \pm 2.7$ & $23.0 \pm 4.7$ \\
\hline Peak disease score & $3.6 \pm 0.2$ & $3.3 \pm 0.1$ & $3.3 \pm 0.1$ \\
\hline Disease burden (\% untreated) & 100 & $51.7 \pm 11.0$ & $50.7 \pm 18.2$ \\
\hline Maximum score & 5 & 4 & 4 \\
\hline
\end{tabular}

Drug treatments $=$ paclitaxel $(20 \mathrm{mg} / \mathrm{kg})$ or docetaxel $(10 \mathrm{mg} / \mathrm{kg})$ on $\mathrm{d} 0-4$ or $6-10$ p.i.. Total mice used is indicated in parentheses. Day of onset peak disease score are calculated mean \pm SEM of sick mice only. Disease burden was calculated as mean \pm SEM percent area under the curve of drug treated relative to untreated mice for individual experiments. No significant difference between drug treated compared to untreated mice was observed.

Table 3.2: Paclitaxel and Ixabepilone alter EAE disease parameters

\begin{tabular}{|l|l|l|l|}
\hline Drug Treatment & Untreated & Paclitaxel & Ixabepilone \\
\hline Incidence & $100 \%(35)$ & $90 \%(20)$ & $80 \%(19)$ \\
\hline Mortality before disease onset & $0 \%$ & $0 \%$ & $0 \%$ \\
\hline Day of onset & $13.86 \pm 0.6$ & $22.78 \pm 1.0 * *$ & $21.93 \pm 1.0 * *$ \\
\hline Peak disease score & $3.9 \pm 0.1$ & $3.8 \pm 0.1$ & $3.3 \pm 0.3 *$ \\
\hline Disease burden $(\%$ untreated) & 100 & $50.52 \pm 6.9 *$ & $55.38 \pm 13.0 *$ \\
\hline Maximum score & 5 & 4.5 & 4 \\
\hline Days at peak disease & $5.8 \pm 0.2$ & $2.6 \pm 0.2$ & $3.1 \pm 0.7$ \\
\hline
\end{tabular}

Drug treatments $=$ paclitaxel $(20 \mathrm{mg} / \mathrm{kg})$ or ixabepilone $(10 \mathrm{mg} / \mathrm{kg}) \mathrm{d} 6-12$ p.i. QOD. Total mice used is indicated in parentheses. Day of onset and peak disease score are calculated as mean \pm SEM of sick mice only. Disease burden was calculated as mean \pm SEM percent area under the curve of drug treated relative to untreated mice for individual experiments. $* \mathrm{P}<0.05, * * \mathrm{P}<0.01$ ANOVA with Dunnett's post-test (drug treated compared to untreated). 

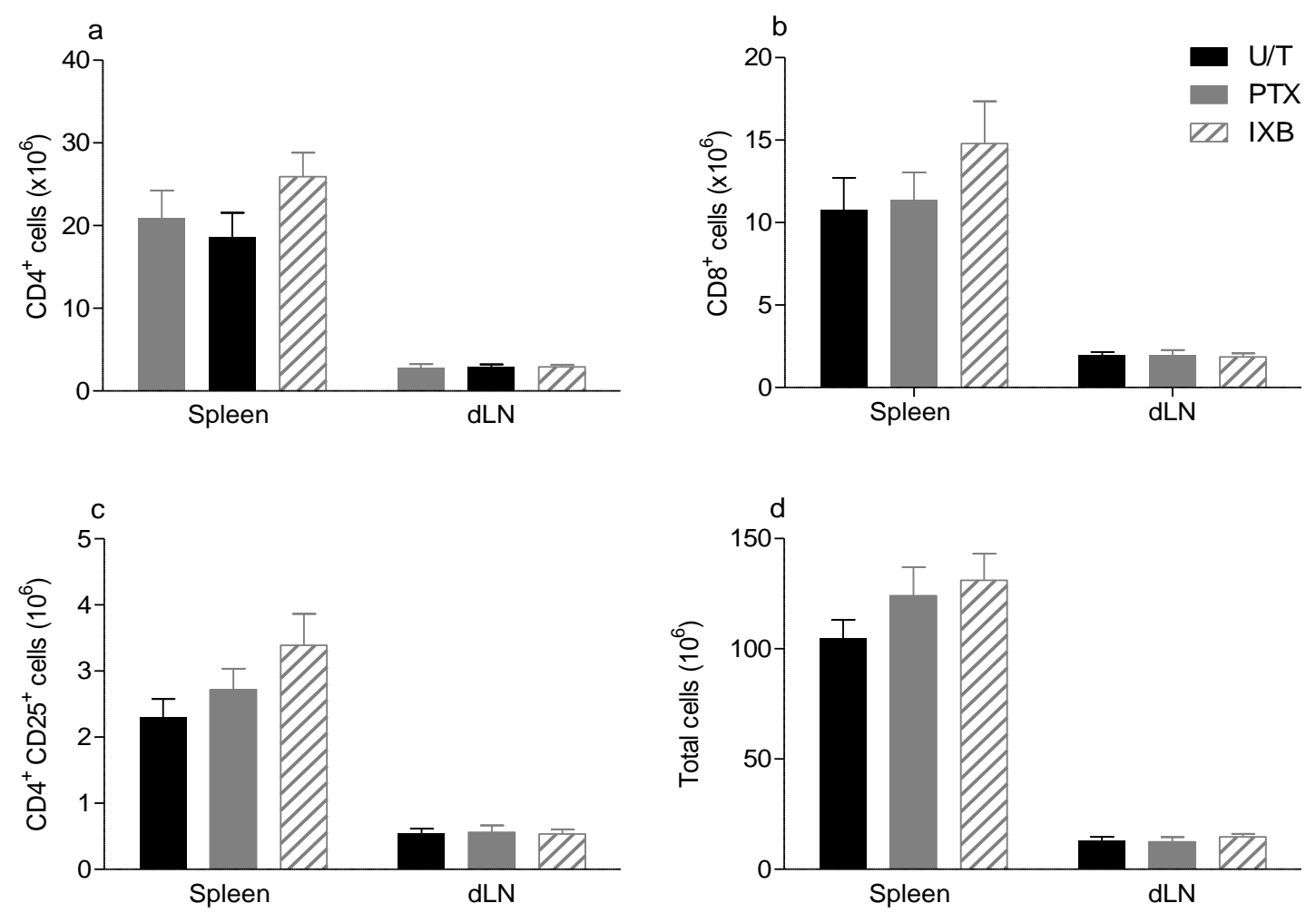

Figure 3.12 Ixabepilone treatment (d6-12 p.i.) does not chronically alter spleen or lymph node cellularity

C57BL/6 mice were immunised for EAE and treated with paclitaxel $(20 \mathrm{mg} / \mathrm{kg})$ or with Ixabepilone $(10 \mathrm{mg} / \mathrm{kg})$ on days 6-12 QOD. Splenocytes and lymphocytes were isolated 30 or 40 days p.i. counted and analysed by flow cytometry to calculate total $\mathrm{CD} 4^{+}(\mathrm{a}), \mathrm{CD}^{+}(\mathrm{b}), \mathrm{CD}^{+} \mathrm{CD} 25^{+}$(c) and total cell numbers (d). Shown are means and SEM of individual mice from two experiments $(n=10$ mice/group). There were no significant differences between treatment groups within spleen or dLN analysed by one-way ANOVA with Newman-Keuls Multiple Comparison test.
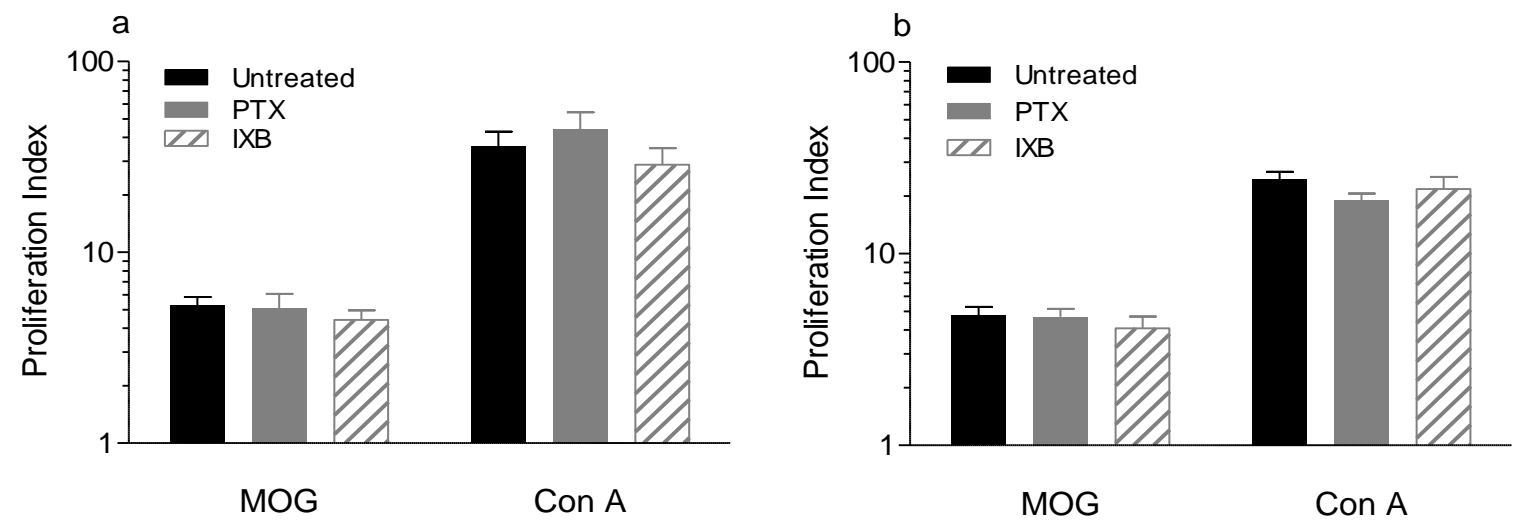

Figure 3.13 Ixabepilone (d6-12 p.i.) does not inhibit ex vivo antigen-specific proliferation C57BL/6 mice were immunised for EAE and treated with paclitaxel $(20 \mathrm{mg} / \mathrm{kg})$ or with Ixabepilone $(10 \mathrm{mg} / \mathrm{kg})$ on days 6-12 QOD. Spleen or lymph node cells were cultured in medium alone or in the presence of Con A $(3 \mu \mathrm{g} / \mathrm{ml})$ or MOG $(27 \mu \mathrm{g} / \mathrm{ml}), 30$ or 40 days p.i.. Proliferation was assessed by ${ }^{3}[\mathrm{H}]$ thymidine $(1 \mu \mathrm{Ci})$ incorporation and is expressed as a proliferation index; expressed as CPM of stimulated wells/CPM of media alone. Shown are means and SEM of individual mice from three experiments ( $\mathrm{n}=10$ PTX, 10 IXB and 15 untreated). No significant differences were observed between groups within each of the stimulation concentrations by one-way ANOVA with Newman-Keuls Multiple Comparison test. 


\subsubsection{Treating with ixabepilone after disease onset}

To explore if administering IXB after disease onset would alter disease progression, 4 doses of IXB (10 mg/kg QOD) were administered to immunised mice following disease onset (d11 p.i.). During drug treatment disease symptoms progressed to a similar severity as to untreated mice and no reduction in peak disease scores occurred (Figure 3.14a). However, by d30 p.i. the IXB treated mice began to exhibit reduced disease severity and increased weight gain compared to those in the untreated group (Figure 3.14a and b). These results suggest that IXB may be effective at limiting the severity of disease when given after onset. However, as this IXB treatment regime was only used in one experiment, further experiments are needed to confirm this.
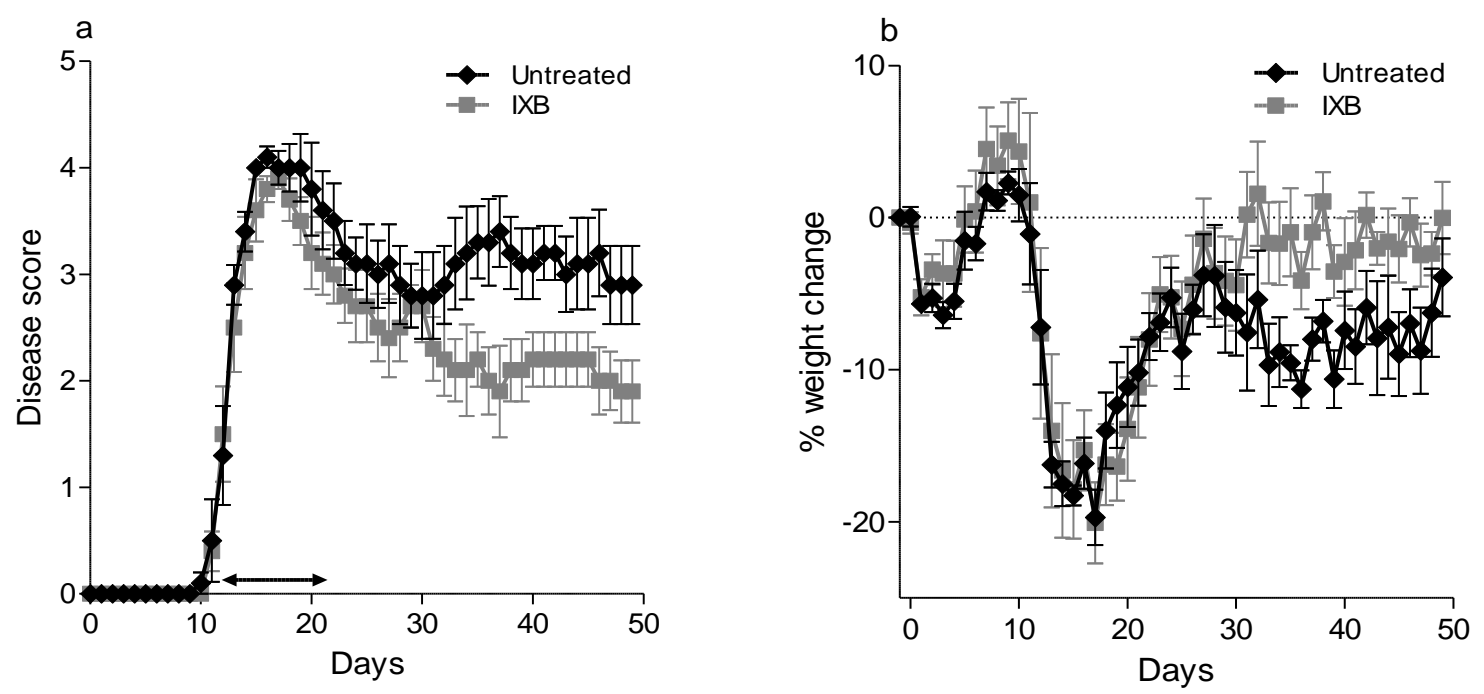

Figure 3.14 Ixabepilone reduces disease severity when administered after EAE onset

Mice were immunised for EAE and treated after with Ixabepilone (10 mg/kg QOD) after disease onset (d 11: shown by arrows). (a) Disease score of all mice (b) \% weight change; calculated as $\%$ change of individual mice from d0. Shown are means and SEM of individual mice from one experiment $(\mathrm{n}=5$ mice per group). 


\subsubsection{Paclitaxel and ixabepilone reduce disease expression in a Balb/c EAE model}

In Balb/c mice, EAE manifests in a different form to that of C57BL/6 and is characterised by lower peak disease scores, reduced incidence and complete disease resolution. To test if MSD would also be effective in this model, Balb/c mice were administered with either PTX $(20 \mathrm{mg} / \mathrm{kg})$ or IXB (10 mg/kg) using a d6-12 p.i. QOD treatment regime. Mice treated with IXB exhibited no disease (Figure 3.15a and b). One mouse treated with PTX had disease symptoms that were delayed in onset and resolved faster than untreated sick mice (Figure 3.15a and b). However, despite the inhibition of disease, significant toxicity was observed with $55 \%$ of PTX treated mice and $25 \%$ of IXB treated mice dying (on d1315 p.i.). These results indicate that MSDs can prevent EAE in Balb/c mice, although this mouse strain appears to be more susceptible to the toxic effects of MSD treatment during EAE.
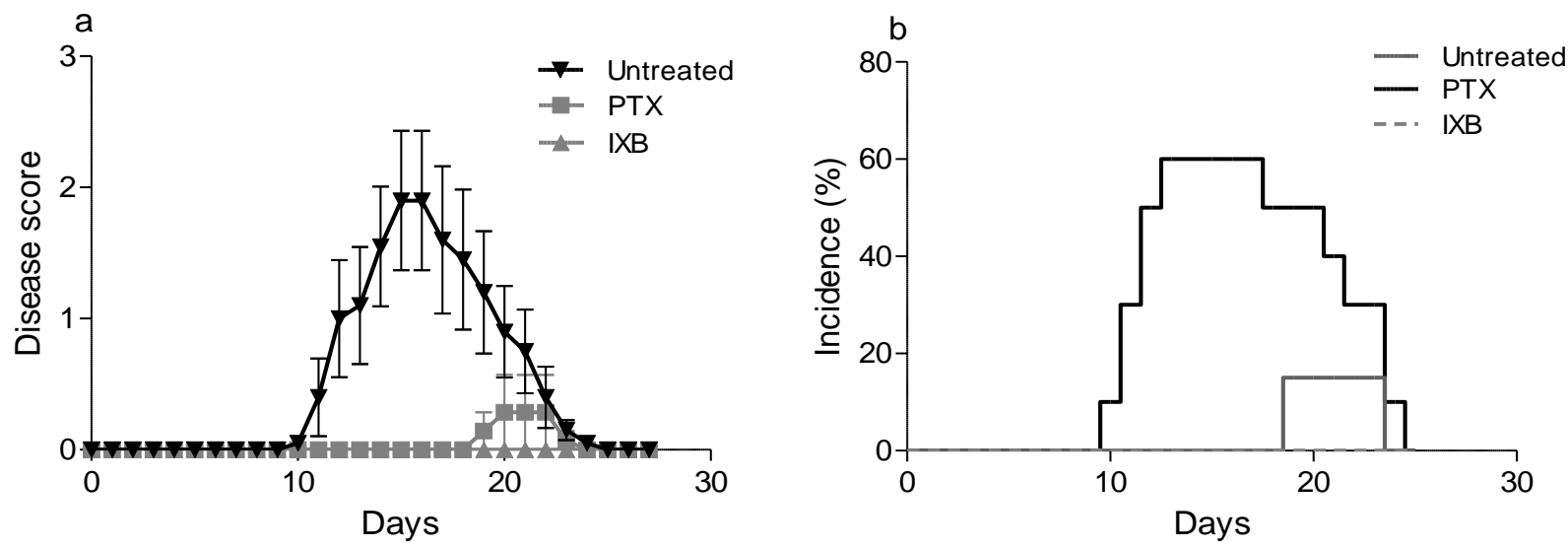

Figure 3.15 Paclitaxel or Ixabepilone inhibits the development of EAE in Balb/c mice Balb/c mice were immunised for EAE and treated with paclitaxel $(20 \mathrm{mg} / \mathrm{kg})$ or with Ixabepilone $(10 \mathrm{mg} / \mathrm{kg})$ on days 6-12 QOD. (a) Disease score of all mice (b) Disease incidence; calculated as percent of mice that develop clinical disease. Data represents two experiments $(n=7$ PTX, 3 IXB and 10 untreated mice per group). Any mice that died were excluded from the analysis. 


\subsubsection{Paclitaxel inhibits disease expression in a relapsing-remitting EAE model}

An alternate model of EAE that more closely resembles the relapsing-remitting form of MS uses SJL/J mice, which are susceptible to EAE induction using the proteolipid protein peptide 139-151 (PLP). To test if PTX could effectively inhibit relapses after initiation of disease, mice were treated with 4 doses of PTX (20 mg/kg, QOD), when the mice began to remit (drop below a disease score of 2) from their initial episode of paralysis. Disease symptoms of all the mice treated with PTX completely resolved following treatment and relapse was delayed for a minimum of 14 days (Figure 3.16a, c, and d). Drug treatment did not cause weight loss and there was also no difference in weight gain during disease remission between treated and untreated mice (Figure 3.16b). This effectiveness of treating after disease onset, provides further evidence of the potential applicability of using MSDs to treat MS as it is likely that in a clinical setting, this would be the time these drug could be administered to patients. 

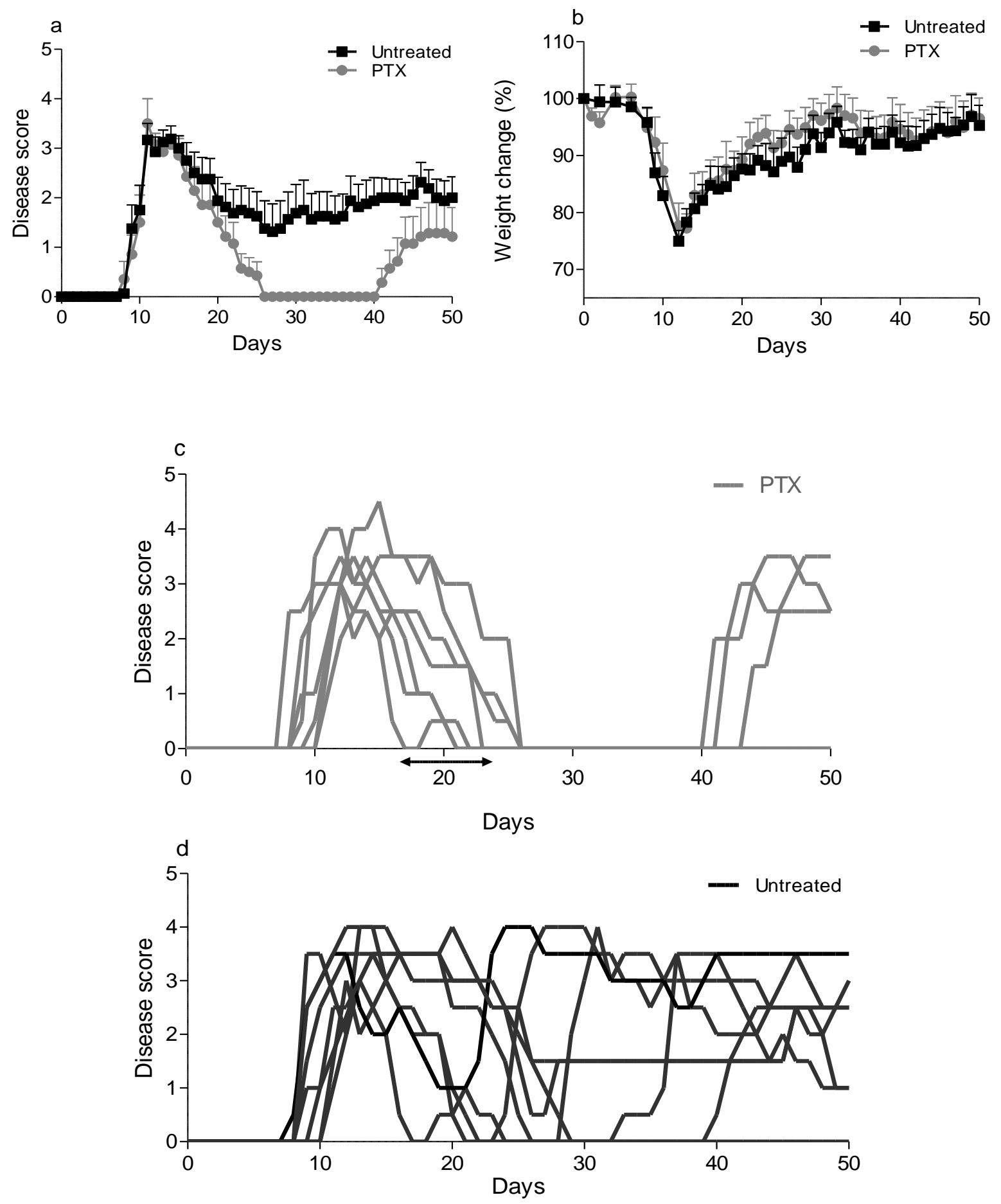

Figure 3.16 Paclitaxel reduces disease expression in a relapsing-remitting EAE model

$\mathrm{SJL} / \mathrm{J}$ mice were immunised for EAE and treated with paclitaxel ( $4 \mathrm{x} 20 \mathrm{mg} / \mathrm{kg}$ QOD) during remission of disease (indicated as a disease score $\leq 2$ ) or left untreated. (a) Disease score of all mice, (b) \% weight change: calculated as $\%$ change of individual mice from d0, (c) Disease score of individual PTX treated mice (arrow indicates time range for initiation of treatment), (d) Disease score of individual untreated mice. Data represents two experiments ( $n=7-8$ mice per group). 


\subsection{Discussion}

Previous work conducted in our laboratory had found that PTX when administered at the MTD of $20 \mathrm{mg} / \mathrm{kg}$ on days 0-4 following immunisation delayed EAE onset and reduced disease incidence (Crume et al., 2009). The current study aimed to further optimise the use of this treatment regime and explore the potential of other MSDs for the treatment of EAE. When the original experiments using PTX were replicated in the present study a similar effect was observed with a delay in disease onset in drug treatment groups. In the DTX or PTX treated mice, total EAE disease burden was reduced; however, this reduction was due to a delay in onset rather than a sustained reduction in disease severity. When peripheral $\mathrm{T}$ cell responses were measured, no reduction in antigen-specific responses occurred in splenocytes or lymphocytes from drug treated animals. In fact, proliferative responses in splenocytes from drug treated animals were increased relative to untreated splenocytes when stimulated with Con A or MOG. PTX and DTX had a very similar effect on the disease response suggesting there is little difference in the activity of these drugs in the EAE model. The delayed disease response but unaltered disease severity, combined with no observed reduction in peripheral antigen-specific proliferation, suggest that drug treatment results in only a transient alteration in immune response or the effect of the MSDs occurs though other mechanisms not detected in the analysis of peripheral immune responses.

Both the current study and previous research has found that PTX administered at the MTD of $20 \mathrm{mg} / \mathrm{kg}$ on d0-4 p.i. causes fatal toxicity in some mice between d7-12 p.i. (Crume, 2007). A study in Lewis rats found that PTX when administered at a MTD on days 6, 8 and 12 p.i. was effective at suppressing EAE but also caused mortality in 11 out of 15 rats (Cao et al., 2000). Interestingly this toxicity is only seen in EAE immunised animals, as the same dosing regime in non-immunised mice caused no obvious alteration in weight or appearance (Crume, 2007). The timing of the toxicity coincides with onset of disease (in non-drug treated mice). It is possible, therefore, that this toxicity could be associated with the inflammatory process that occurs during disease induction. 
Development of EAE undergoes a documented progression of immune events beginning with the immunisation of a myelin based antigen. During early stages of the disease process, antigen presenting cells (APC) acquire the antigen from the immunisation site, while simultaneously being activated by 'immune danger signals' present in the immune adjuvant, CFA. Following migration to the draining lymph node, APCs present this antigen to antigen-specific $\mathrm{T}$ cells, which then undergo the process of clonal expansion. This proliferation occurs early in the disease process and peaks within the periphery by d8-9 p.i. (Hofstetter et al., 2010; Targoni et al., 2001). Usually, from about day 7 onwards, activated $\mathrm{T}$ cells can begin to migrate into the CNS, and disease symptoms start to appear (Juedes et al., 2000). During this period higher than normal concentration of inflammatory cytokines are expressed in immunised mice (Hofstetter et al., 2010).

It was initially thought that PTX, acting on TLR-4 receptors, could be further enhancing the inflammatory milieu to a toxic level. If this were the cause of the toxicity, DTX, an analogue of PTX that does not activate TLR-4 receptors, would not cause any mortality. However, DTX given to EAE immunised mice on d0-4 p.i caused a similar level of mortality to that of PTX treated animals (Table 3.1). This indicates that TLR-4 activation is unlikely to be the cause of MSD toxicity in immunised animals. As with the toxicity, there also appears little obvious difference in the disease modifying effects between DTX and PTX, indicating that the TLR-4 activity of PTX confers no obvious advantage or disadvantage to either the effectiveness or the toxicity of MTD in EAE.

Treating at d6-10 p.i. with either PTX or DTX also caused a similar rate of mortality between $\mathrm{d} 7-12$ p.i., further supporting the concept that the toxicity may be related to the inflammatory events occurring during the drug vulnerable period. The variability in the occurrences of toxicity may be related to the specific kinetics of the inflammatory process as they occurr in individual mice and that toxicity appears only when drug treatment coincides with particular disease related events. A number of additional factors support hypothesis. Mortality of drug treated mice was only observed in about half the experiments using either the d0-4 or d6-10 p.i dosing regimen, and when toxicity did occur it did so only in a subset of drug treated animals. Additionally, no mortality occurred in mice that 
were administered PTX on d21-25 p.i. This treatment time point is likely to be after peak inflammatory activity in the periphery has occurred (Kuerten et al., 2010; Targoni et al., 2001).

A potential cause of the toxicity that could explain the unusual combination of high drug concentration and inflammation could be pneumonitis or a more generalised pulmonary oedema. These adverse events would be consistent with the symptoms of dyspnea and hunched appearance in mice before they died. There are many reports in the literature of cancer chemotherapy patients having severe pulmonary oedema and/or pneumonitis, days to weeks after PTX or DTX treatment (Dimopoulou et al., 2006; Fujimori et al., 1998; Ramanathan et al., 1996; Semb et al., 1998; Wang et al., 2001). While severe pneumonitis is a rare side effect of taxane treatment, it is a life threatening condition that often requires respiratory ventilation (Nagata et al., 2010).

When CFA is injected intravenously in animals it can induce an experimental form of pneumonitis on its own (Edwards et al., 1983; Soler et al., 1975). While the administration route would assist in this process (as it is likely the CFA emulsion will lodge in the capillaries of the lungs), it is interesting to note that CFA causes an inflammatory response that can result in pneumonitis. In rodents PTX and DTX have been shown to lower interstitial fluid pressure which results in fluid flux, protein extravasation and tissue oedema (Borge et al., 2006; Bronstad et al., 2004). Additionally, it has been found that pulmonary oedema can occur transiently after i.v. injections of PTX in rats. This was correlated to increased levels of substance $\mathrm{P}$ and could be inhibited by antagonism of neurokinin receptors. (Itoh et al., 2004; Sendo et al., 2004). Substance P acting thought neurokinin receptors causes vasodilatation and increased vascular permeability and can also can act as a chemoattractant to lymphocytes monocytes and neutrophils (O'Connor et al., 2004).

If increases in vascular permeability were occurring due to PTX administration, the effects are likely to be acute and would occur when drug concentrations are high. It is conceivable that prolonged high drug concentrations causing increased capillary permeability and 
potential oedema could initiate a pulmonary inflammatory response. The inflammatory response that results in lethal pneumonitis may be delayed until drug concentrations are low enough to allow for immune cell extravasation or until a large enough response has occurred. This effect may only occur, therefore, in drug treated immunised mice since the inflammatory milieu may exacerbate this effect to an extent that results in the toxic effects observed. Alternately a similar mechanism of increased vascular permeability and oedema caused by MSD administration could be directly affecting the CNS. No investigation was conducted in the current study to determine if oedema was occurring within the brain or lungs following drug treatment. Further in-depth research would need to be carried out to explore this hypothesis. As it was found that spacing the doses out by treating every second day did not cause any mortality C57BL/6 mice, this treatment regime was focused on for future experiments and the cause of toxicity was not further investigated.

Similar to the day 0-4 p.i. treatment, MTD doses of PTX and DTX administered on days 610 p.i. delayed disease onset and reduced disease incidence. As the initial induction of peripheral inflammatory responses occurs within the first week following immunisation (Hofstetter et al., 2010; Targoni et al., 2001), the alteration of disease following d6-10 p.i. drug treatment indicates that MSDs can alter pre-established autoimmune activity. It is interesting that treatment on either d0-4 p.i. or d6-10 p.i. resulted in a similar delay in disease onset. If the alterations in disease observed following the d6-10 p.i. treatment were solely due to inhibition of $\mathrm{T}$ cell proliferation, it would be unlikely that the delay in disease onset would be as prolonged as that seen in d0-4 p.i. treatment. By day 6 p.i., clonal expansion of MOG reactive T cells has begun to occur, and antigen-specific responses can be detected within the dLN and spleen (Crume et al., 2009). Thus a large population of autoreative $\mathrm{T}$ cells would already be present in the secondary lymphoid organs, and MSD treatment on d6-10 p.i. is unlikely to cause ablation of these pre-established populations.

If disease responses can be modified after initial inflammatory events, it was thought multiple dose regimes might confer improved disease modification. However when a dual dosing regime was used, little benefit in reducing disease burden was gained compared to a single dosing regime. Within the d21-26 treatment groups, mice that had not already 
displayed disease symptoms had an increased delay in disease onset. In mice that already exhibited disease symptoms, this treatment time point would be after the majority of myelin reactive immune cells had already migrated from the periphery to the CNS (Flugel et al., 2001; Furtado et al., 2008). Both PTX and DTX have low permeability for the blood-brain barrier (BBB) and drug concentration in the CNS are very low compared to plasma levels (Fellner et al., 2002; Kemper et al., 2004). As the BBB can become leaky during EAE, it is possible PTX or DTX may more readily enter the CNS after disease onset. However, given both of these drugs are substrates for drug efflux pumps (which are highly expressed on CNS endothelial cells) the concentrations of drug within the CNS after disease onset is still likely to be much lower than that occurring in peripheral tissues (Fisher et al., 1996; Loscher et al., 2005). The lack of disease modification following disease onset indicate that PTX and DTX are only effective on the peripheral immune response and not that which is occurring within the CNS.

Interestingly, treating mice with IXB after disease onset resulted in a reduction in disease severity. This contrasts the above results where the dual dosing of PTX or DTX did not alter disease burden. Additionally, a previous study had found that PTX administered at the peak of disease $(20 \mathrm{mg} / \mathrm{kg} / \mathrm{d}$ for 5 consecutive days) had no effect on disease burden (Crume, 2007). One possible explanation could be that IXB was having an effect within the CNS, as unlike the taxanes, IXB passes more readily into the CNS (Brunden et al., 2011). However, as this IXB treatment regimen was only used in one experiment and was not compared directly to PTX, it is possible that this is not a typical effect of the drug. While not explored further in the present study future investigation into the effect of IXB after disease onset (i.e. repeating the experiment in section 3.3.6) may be warranted.

A similar delay in disease onset and reduction in disease burden was seen between IXB and PTX treatments administered on d6-12 p.i. QOD (Table 3.2). The initial pilot experiment using IXB showed particularly impressive inhibition of disease. The disease severity in untreated immunised mice within this initial experiment was mild (mean peak score 3.3) compared to subsequent experiments (mean peak score 4.1), and disease onset also occurred at a later time point (d16 p.i. vs. d12.4 p.i.). This may indicate that the 
efficacy of IXB treatment is related to the severity and/or the kinetics of disease expression. When multiple experiments were compared, disease protection in drug treated mice (PTX and IXB, d6-12 p.i. QOD) was correlated to disease severity of unimmunised mice ( $\mathrm{r}=$ $0.724, \mathrm{p}=0.0137)$. Thus, if disease severity in unimmunised mice is high, protection induced by drug treatment is reduced (Figure 3.17b). A similar (but non-significant), trend was also seen in PTX and DTX (d0-4 p.i. and d6-10) treatment (Figure 3.17a). This suggests that MSD may be more effective in a less aggressive inflammatory environment. However, irrespective of the disease severity, when the disease burden (as measured by area under the curve; AUC) of MSDs treated mice was compared to disease burden of untreated mice, it can be seen that all the MSDs tested provided protection against disease in each experiment (Figure 3.17). 

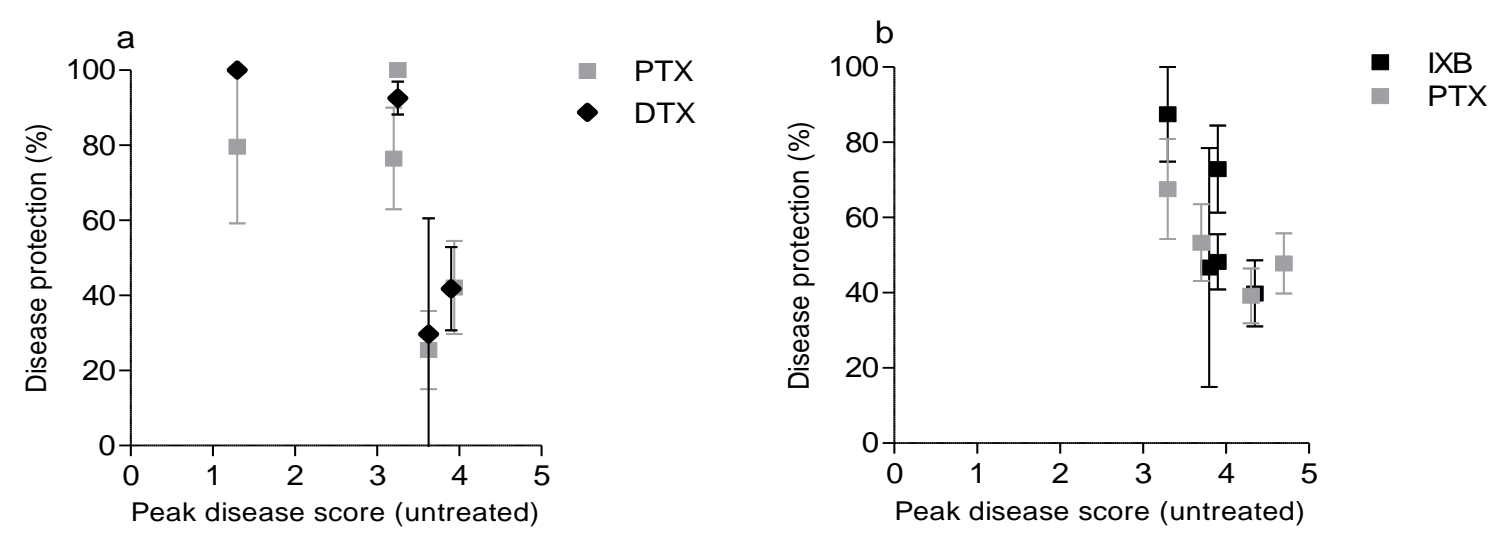

Figure 3.17 Paclitaxel, docetacel or ixabepilone treatment is more protective in less severe disease

Disease protection in drug treated C57BL/6 mice compared to mean peak disease score of untreated mice within experiments. Disease protection (a) PTX $20 \mathrm{mg} / \mathrm{kg}$ or DTX $10 \mathrm{mg} / \mathrm{kg}$ (administered on d0-4 or 6-10 p.i.). (b) PTX $20 \mathrm{mg} / \mathrm{kg}$ or IXB $10 \mathrm{mg} / \mathrm{kg}$ (administered on d6-12 p.i. QOD). Disease protection calculated as AUC untreated mice - AUC drug treated mice / AUC untreated mice. Each data point represents a single experiment shown as mean and SEM of individual mice. Any mice that died were excluded from the analysis.
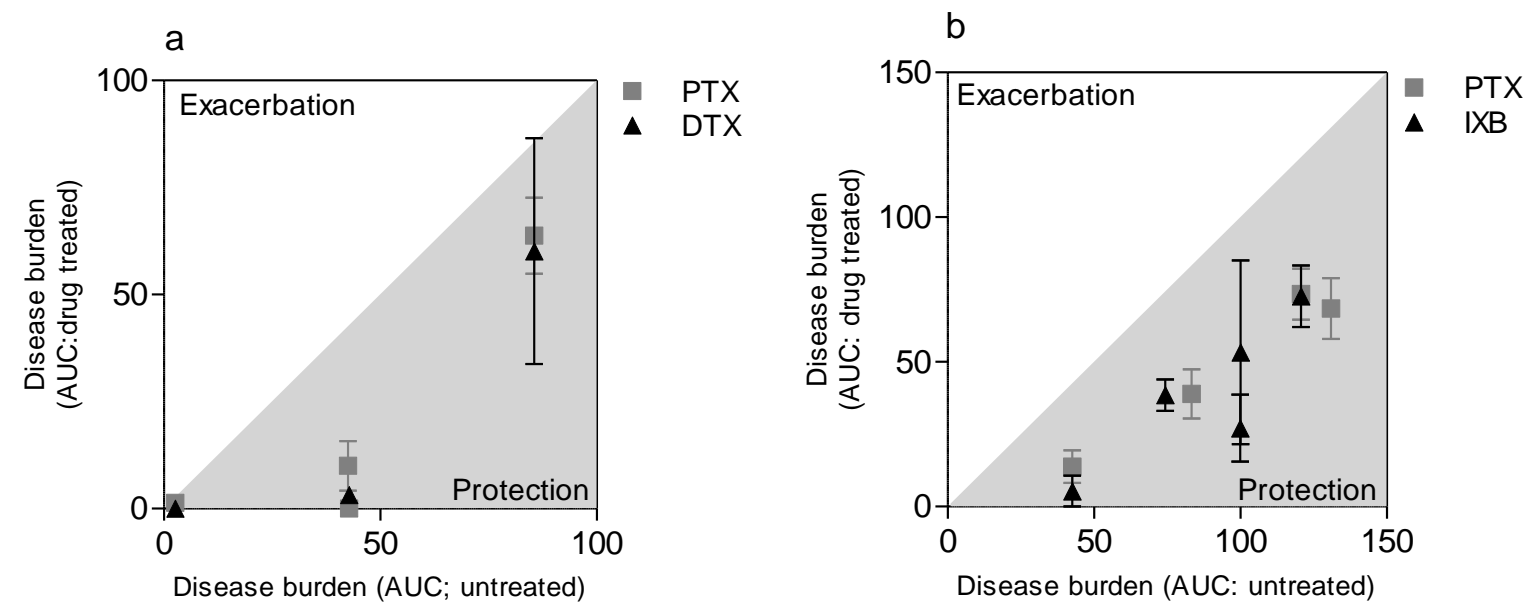

Figure 3.18 Paclitaxel, docetacel or ixabepilone treatment has a protective effect in EAE

Protection or exacerbation of EAE for drug treated vs. untreated C57BL/6 mice; calculated as disease burden (AUC: area under the curve) of individual drug treated mice compared to untreated mice. (a) PTX $20 \mathrm{mg} / \mathrm{kg}$ or DTX $10 \mathrm{mg} / \mathrm{kg}$ (administered on d0-4 or 6-10 p.i.). (b) PTX $20 \mathrm{mg} / \mathrm{kg}$ or IXB $10 \mathrm{mg} / \mathrm{kg}$ (administered on d6-12 p.i. QOD). Each data point represents a single experiment shown as mean and SEM of individual mice. Any mice that died were excluded from the analysis. 
In the initial experiment only 1 of 5 mice treated with IXB exhibited disease symptoms, and no overt changes in cellularity or antigen-specific responses were seen in splenocytes or lymphocytes from the IXB treated mice. Thus, even when disease was not apparent, MOG specific T cells were present in drug treated mice, and these cells were as responsive to antigen stimulation as those of untreated mice. Therefore, it is likely that drug treatment is either not inhibiting the autoreactive $\mathrm{T}$ cell response or is only doing so transiently. The fact that robust antigen responses were seen in IXB treated mice that did not exhibit disease symptoms suggests that the alteration of disease in drug treatment is not related to the anti-mitotic effects of MSDs. However, as the MOG/CFA depot is still visible at the injection site months after immunisation it is likely that even if MSDs were inhibiting clonal expansion or antigen-specific responses during or shortly after drug treatment, longterm responses could potentially develop well after the drugs had been administered. To test if in fact this is occurring, a passive induction model of EAE could be used, since in this model encephalogenic cells are adoptively transferred into recipient mice, removing the need for immunisation.

When a number of experiments were compared, a similar delay in disease onset and reduction in disease burden was seen between IXB and PTX treatments; however, IXB treatment also resulted in a significant reduction in peak disease score that was not seen with PTX (untreated $=3.9+/-0.1$ vs. IXB $=3.3+/-0.3$ ) (Table 3.2). While an overall reduction in average peak disease severity of 0.6 does not appear substantial, this reduction translates into considerable gains in functionality for IXB-treated mice. In terms of clinical symptoms, this difference equates to hind limb weakness as opposed to full hind limb paralysis. Likewise a maximal disease score of 4 for IXB compared to 4.5 for PTX and 5 for untreated mice is a substantial improvement. These factors combined with the potential disease modifying effects observed when the drug is given after disease onset indicate that IXB provides a small therapeutic advantage over PTX within the context of EAE.

In contrast to the C57BL/6 mice, Balb/c mice treated with IXB or PTX on d6-12 p.i. QOD exhibited little or no disease. A factor to consider in assessing the difference between the disease inhibition seen in the two strains is in the drug treated mice is likely to be 
associated with the difference in disease expression between the two strains. The Balb/c EAE model exhibits a monophasic disease followed by complete remission of symptoms, whereas the C57BL/6 mice have a more severe disease peak followed by a chronic but less severe plateau. If MSDs cause a transient alteration in inflammatory responses, this might explain why inhibition of disease is far more pronounced in the Balb/c model compared to the C57BL/6 model.

Despite the inhibition of disease in Balb/c mice 55\% of PTX and 25\% of IXB treated mice died. It is possible that the substantial disease suppression in drug treated mice could be due to more potent effects of the drugs in Balb/c mice (as indicated by the increased toxicity). However, this is unlikely, since, no correlation was found between toxicity and drug efficacy (number of deaths vs. untreated groups peak disease scores; $\mathrm{r} 0.171, \mathrm{p}=$ 0.686; data from 5 separate experiments) within experiments using C57BL/6 mice. Another possible explanation for the increased toxicity in Balb/c mice could be due to strain differences in sensitivity to the drug or susceptibility to the side effects. The Balb/c mice weighed on average 50\% more than the C57BL/6 mice, and the drug concentrations were increased accordingly to give the same $\mathrm{mg} / \mathrm{kg}$ dose as was used for C57BL/6 mice. As no unimmunised Balb/c mice were injected with these doses, it cannot be ruled out that the dose was too high and would have caused significant toxicity to unimmunised mice.

Previous studies conducted in our laboratory using PTX have found that high concentrations of PTX are required to modify disease (Crume, 2007). The study by Cao et al. in Lewis rats found that PTX could modify EAE in Lewis rats when administered at a MTD on day 6, 8 and 12 p.i., but this dose also caused significant mortality (Cao et al., 2000). Interestingly, when this experiment was replicated in mice, no mortality but also little alteration in disease progression was observed (Crume, 2007). Administering a MTD at $\mathrm{d} 6,8,12$ p.i. is very similar to the effective dosing of $20 \mathrm{mg} / \mathrm{kg}$ on $\mathrm{d} 6-12$ QOD used in the current study. Collectively, these factors indicate that MSDs have a narrow therapeutic window with administration of high concentrations over a prolonged period required to effectively alter disease. The deaths observed in C57BL/6 mice treated daily and the in QOD treatmented Balb/c mice emphasise the narrow range between efficacy and toxicity. 
While the toxicity at least within the C57BL/6 model appears to be EAE specific, it is also dose related. Toxicity in this model only occurred in mice treated using a daily drug dosing. Treating with a QOD regime may allow for drug concentrations to be maintained within the therapeutic window without causing peaks in concentration that, combined with the inflammatory environment, induce unwanted toxicity.

Treating SJL/J mice with PTX after signs of disease remission resulted in a delay in relapse onset and a reduction in incidence. As relapses did occur, however, the PTX treatment was clearly not fully ablating the encephalogenic immune response. Unlike the Balb/c mice that have a single episode of disease, SJL/J mice exhibit waves of disease expression that are correlated with fresh influx periods of myelin reactive $\mathrm{T}$ cells, migrating into the CNS (Targoni et al., 2001; Vanderlugt et al., 2000). It seems likely PTX is inhibiting this influx of $\mathrm{T}$ cells. This could be due to either inhibition in migration of these cells into the CNS or transiently depleting the encephalogenic T cells to a level that is ineffectual at causing a relapse.

Within the context of MS, in humans, any therapy would generally be administered after development of symptoms, as a prophylactic treatment is obviously not feasible. The disease modifying effects of PTX within the SJL/J model provides further evidence for the applicability of using MSDs for the treatment of MS. However, it is likely, given the results discussed above, the therapeutic effect of MSD in MS would be to lessen disease relapse or severity rather than to completely inhibit the disease.

Many of the experiments conducted within this chapter were exploratory in nature and were focused on further optimising the dosing schedule of MSDs for the treatment of EAE. As such, some of these experiments were only conducted once or twice. Additionally, although most experiments began with 5 mice per group, due to the toxicity of some of the treatment regimes the final mouse numbers were often less than this. These factors need to be considered when interpreting the findings from some of the experiments within this chapter. However, while these factors may increase the potential variability, when the experiments are viewed collectively (Table 3.1 and 3.2; Figure 3.17 and 3.18), or alongside 
previous research (Crume, 2007; Crume et al., 2009) consistent trends emerge, which have been summarised below.

\subsection{Summary}

The findings from these experiments show that MSDs can effectively alter the disease expression of EAE. High drug concentrations and prolonged drug exposure appear to be necessary for these disease-modifying effects. Drug toxicity in MSD-treated immunised mice is unlikely to be due to TLR-4 activation by PTX, and this toxicity can be avoided by altering the MSD treatment regimen. The lack of evidence for significant chronic alterations in cellularity and cytokine expression with all three drugs suggest that if MSDs cause any modulation of the immune system, this alteration only occurs during or soon after drug exposure. 


\section{Chapter 4:}

\section{Identifying microtubule-stabilising drugs mechanisms of action}




\subsection{Introduction}

It was shown in the previous chapter that MSD effectively alter EAE disease expression. However, the mechanism responsible for these alterations and the potential involvement of the immune responses in this mechanism are not well defined. While a number of studies have documented the effects of MSDs on inflammatory diseases including arthritis, psoriasis, systemic lupus and EAE, little is known about the direct effect on immune cell activity (Brahn et al., 1994; Cao et al., 2000; Ehrlich et al., 2004; Oliver et al., 1994; Song et al., 1998).

The most obvious possible mechanism by which MSD treatment reduces inflammatory diseases is inhibition of proliferation. Diseases such as EAE are mediated by the activation and proliferation of self-reactive lymphocytes, and inhibition of these events would be expected to alter disease expression. For example, PTX has been shown inhibit in vitro proliferation of stimulated lymphocytes (Brown et al., 1985; Crume et al., 2007). Additionally, a reduction in leukocytes or 'leukopenia' is a common side effect of cancer chemotherapy (Sako et al., 2004; Tsavaris et al., 2002). These factors suggest that MSDs are likely to inhibit leukocyte proliferation in vivo, and this inhibition could be responsible for the efficacy of these drugs in inflammatory disease models.

However, non-anti-mitotic effects of MSDs on immune cells have also been noted. Administration of PTX can impair the ability of cytotoxic T cells to kill antigen-specific target cells in vitro and in vivo (Chuang et al., 1994; Robinson et al., 2008). While with murine macrophages, cultured under inflammatory conditions, MSDs can inhibit TNF- $\alpha$ and NO production (Crume et al., 2007). It has also been noted that patients undergoing PTX- or DTX-based cancer chemotherapy have increased inflammatory cytokines IL-2, IL-5 and IFN- $\gamma$ levels, but decreased TNF- $\alpha$ (Tsavaris et al., 2002). These results indicate that the effects of MSDs on the immune system are unlikely to be solely due to antiproliferative activity.

In addition to cell division, microtubules are important for a wide range of cellular processes including intracellular transport, cell migration and cell polarity (Hirokawa, 1998; 
Jordan et al., 2004; Nogales, 2001). Microtubule function is required for antigen processing and presentation by dendritic cells and macrophages, and PTX can interfere with these processes (John et al., 2010; Peachman et al., 2004). In macrophages, formation of podosomes, which are necessary for directional movement, and transcelluar diapedesis are microtubule-dependent processes (Calle et al., 2006; Linder et al., 2000). It is therefore conceivable that alterations in these processes may contribute to the disease modifying effects of PTX in the EAE model.

\subsection{Aims and Objectives}

The previous chapter demonstrated that MSDs, administered early in the disease process, had little effect in modifying chronic inflammatory responses. In contrast, this chapter tests the hypothesis that drug treatment modifies disease-specific immune responses at earlier time points in the disease process. Additionally, to better understand the mechanism by which PTX and MSD reduce EAE disease expression, the effects of PTX treatment on immune cell proliferation and migration were determined.

\subsubsection{Specific Aims:}

1. To assess changes in immune responses immediately following drug treatment

2. To determine if proliferation is altered by PTX treatment

3. To identify if PTX treatment alters in vivo immune cell migration 


\subsection{Results}

\subsubsection{Spleen and LN cellularity is altered following paclitaxel treatment immediately after immunisation.}

As was demonstrated in the previous chapter, MSDs effectively delayed EAE disease onset but did not appear to cause significant chronic alterations in immune parameters measured. Thus, it was hypothesised that effects of MSDs on the immune response may be transient, occurring only during or soon after drug administration. To explore this possibility, mice were immunised for EAE and treated with PTX daily from day 0-4 p.i.. On day 5 p.i. the $\mathrm{dLN}$ and spleens were isolated from untreated and treated, unimmunised and immunised mice and changes in cellularity and antigen responses were assessed. Because both PTX and DTX treatment had a very similar effect on the disease response, only PTX was used in these experiments.

Five days after immunisation, total cell numbers were increased in the spleens and dLN of immunised untreated mice (iUT) compared to immunised PTX-treated (iPTX) and unimmunised PTX (uiPTX) or untreated, unimmunised (uiUT) mice (Figure 4.1f). The increase in cell numbers was particularly pronounced in $\mathrm{F} 4 / 80^{+}$and $\mathrm{Gr}-1^{+}$populations within both the spleen and dLN within iUT but not iPTX treated mice (Figure 4.1d and e). Additionally, within the spleens of iPTX mice, there were significantly less total CD $4^{+} \mathrm{T}$ cells compared with iUT (Figure 4.1a). Although there were significantly less $\mathrm{CD}^{+} \mathrm{T}$ cells in iPTX compared to iUT mice, no difference was observed between iUT and uiPTX or uiUT mice. Within the dLN, a significant increase in $\mathrm{CD}^{+} \mathrm{T}$ cells was observed within the iUT mice when compared to all other treatment groups (Figure 4.1a). No difference in total $\mathrm{CD} 4^{+} \mathrm{CD} 25^{+} \mathrm{T}$ cells was observed, indicating the increase in $\mathrm{CD} 4^{+}$iUT mice was due to $\mathrm{CD}^{+} \mathrm{CD} 25^{+} \mathrm{T}$ cells (Figure 4.1b). Finally, no significant differences in $\mathrm{CD}^{+} \mathrm{T}$ cells were observed between the groups (Figure 4.1c) suggesting that the changes in cellularity were cell type specific and not due to generalised depression in leukocyte populations by PTX. 

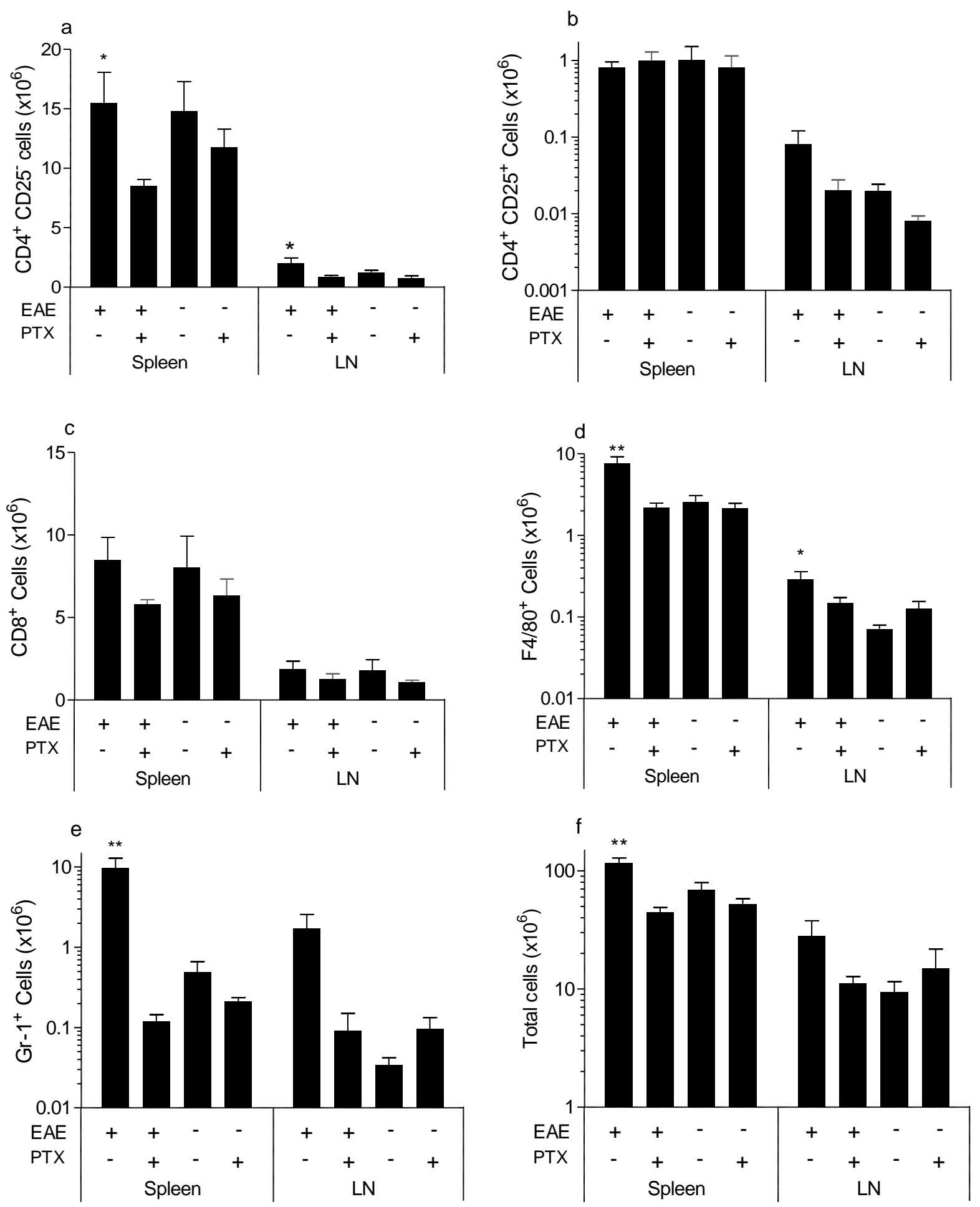

Figure 4.1 Spleen and lymph node cellularity is altered immediately after paclitaxel treatment (d0-4p.i) C57BL/6 mice were immunised for EAE and treated with paclitaxel $(20 \mathrm{mg} / \mathrm{kg})$ or left untreated. Five days p.i. spleen and lymph node cells were isolated, counted, stained for cell surface markers and analysed by flow cytometry. Mean total cell numbers of (a) $\mathrm{CD} 4^{+} \mathrm{CD} 25^{-}$(b) $\mathrm{CD} 4^{+} \mathrm{CD} 25^{+}$(c) $\mathrm{CD}^{+}$(d) $\mathrm{B} 220^{+}$(e) F4/80 $0^{+}$and (f) Gr- $1^{+}$splenocytes and lymphocytes. Shown are means and SEM of values from individual mice from two separate experiments (spleens) or one experiment (dLN) ( $n=5-9$ /group). ${ }^{*} p<0.05$ or $* * p<0.01$ compared to immunised paclitaxel treated mice, by one-way ANOVA with Newman-Keuls multiple comparison test. 


\subsubsection{Antigen-specific responses are impaired in spleen and LN cells following paclitaxel treatment immediately after immunisation}

To identify whether the differences in cellularity between PTX-treated and untreated mice extended to altered cellular responses, ex vivo cell proliferation and cytokine production was measured in spleen and LN cultures. Despite the increase in total CD $4^{+} \mathrm{T}$ cell numbers in the iUT mice, no difference in Con A-induced polyclonal proliferative responses were observed between any of the treatment groups (Figure 4.2a). In contrast, MOG-specific proliferation was inhibited in the spleen and LN cell cultures from iPTX mice compared to iUT mice (Figure 4.2a and b). Little response was detected in cultures of cells from unimmunised mice confirming that the response in immunised animals was antigenspecific.

Nitric oxide (NO) production has been shown to inhibit $\mathrm{T}$ cell proliferation in vitro (Albina et al., 1991; Krenger et al., 1996). Thus, to investigate the possibility that NO was the cause of the reduced antigen-specific proliferation in the cells from PTX immunised mice, the Greiss reaction was used to assess NO levels in the culture supernatants. Because NO is an unstable molecule with a very short half life, the Griess assay utilises the stable NO breakdown product, nitrite, as an indicator of relative NO production. No significant difference in nitrite levels were detected between any of the treatment groups in either the splenocyte or lymphocyte cultures (Figure 4.3a and b) suggesting that high level NO production was not responsible for the reduced proliferation.

To investigate if cytokine production from these cells was affected by PTX treatment, culture supernatants from spleen and LN cells that had been stimulated with Con A or MOG for 48 or 72 hours, respectively, were analysed for IL-1 $\beta$, IL-2, IL-4, IL-5, IL-6, GM-CSF, IL-10, IL-17, IFN- $\gamma$ and TNF- $\alpha$. Of the 10 cytokines measured, only those showing differences have been included in the analysis (Figure 4.4a-f). Strikingly, splenocytes from iUT mice produced IL-6 in response to MOG and polyclonal stimulation while IL-6 production by splenocytes from iPTX treated animals was inhibited both to antigen-specific and polyclonal stimulation (Figure 4.4e). Moreover, this inhibitory effect on IL-6 production extended to the uiPTX treated mice which produced only low levels of IL-6 in response to polyclonal stimulation. In contrast to splenocytes, only low levels of 
IL-6 were detected from LN cells, and there was no significant difference between treatment groups (Figure 4.4f).

In addition to the dramatic effect on IL-6 production, PTX treatment caused a significant reduction in antigen-specific but not polyclonal-induced IFN- $\gamma$ production (Figure 7.4a). A similar trend was observed in the LN cell cultures; however, this trend did not reach statistical significance (Figure 4.4b). In contrast to IFN- $\gamma$, IL-17 production in response to polyclonal stimulation was significantly inhibited in both splenocyte and LN cell cultures of PTX-treated animals (Figure 4.4c and d). Furthermore, no IL-17 was produced by MOG stimulated splenocytes from iPTX mice. Despite these impairments in IL-17 production after PTX treatment, similar levels of MOG specific IL-17 were produced by LN cells from iPTX and iUT mice (Figure 4.4d). Theses results indicate that following PTX treatment, the ability of splenocytes to respond in an antigen-specific manner is inhibited, and although LN cell responses are attenuated, antigen-specific cytokine production still occurs. The finding that antigen-specific responses were observed in the dLNs indicates that drug treatment does not completely ablate autoimmune activity. Additionally, the difference in the response between splenocytes and lymphocytes may indicate that the effects of PTX treatment are more pronounced in the spleen than within the dLNs. 

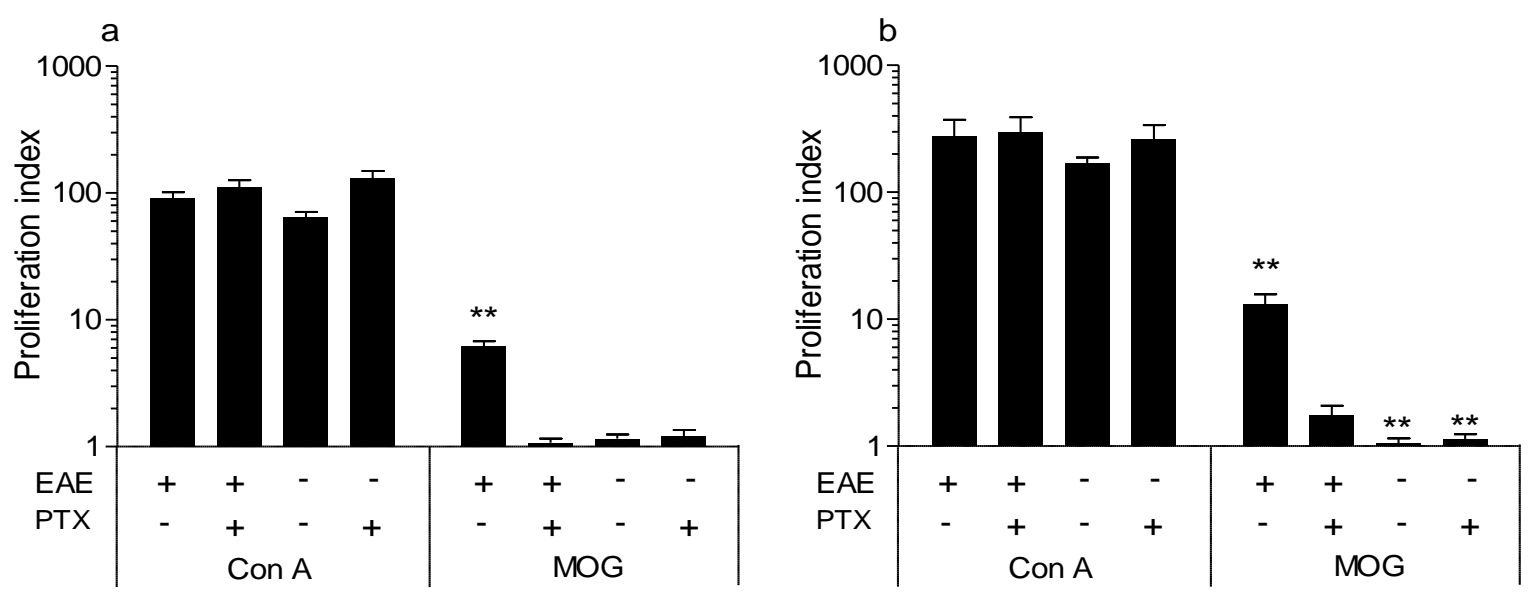

Figure 4.2 Ex vivo antigen-specific proliferation of spleen and lymph node cells is inhibited immediately after paclitaxel treatment (d0-4p.i)

C57BL/6 mice were immunised for EAE and treated with paclitaxel $(20 \mathrm{mg} / \mathrm{kg})$ or left untreated. Five days p.i. (a) spleen and (b) lymph node cells were cultured in the presence or absence of ConA $(3 \mu \mathrm{g} / \mathrm{ml})$ or MOG $(27 \mu \mathrm{g} / \mathrm{ml})$ for 48 or 72 hour respectively. Proliferation was assessed by ${ }^{3}[\mathrm{H}]$ thymidine $(1 \mu \mathrm{Ci})$ incorporation and is expressed as a proliferation index; CPM of stimulated wells/CPM of media alone. Shown are means and SEM of values from individual mice from two separate experiments $(n=5-9$ per group). $* * p<0.01$ compared to immunised paclitaxel treated mice, by one-way ANOVA with NewmanKeuls multiple comparison test.
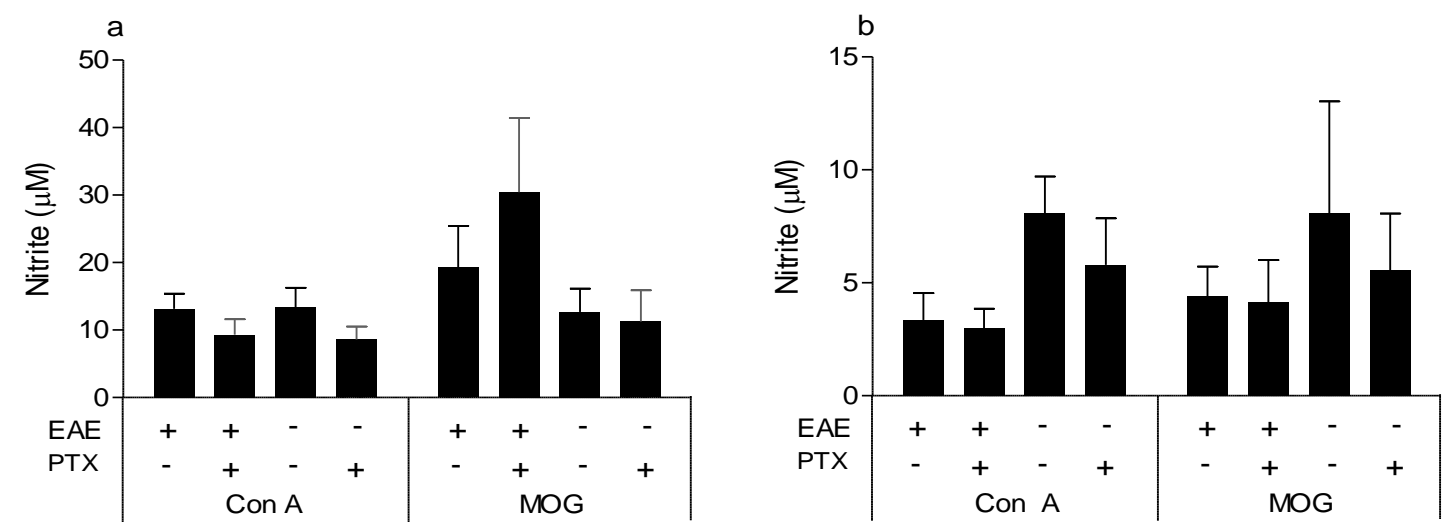

Figure 4.3 Ex vivo nitric oxide production is not altered in spleen and lymph node cells immediately after paclitaxel treatment (d0-4p.i)

C57BL/6 mice were immunised for EAE and treated with paclitaxel $(20 \mathrm{mg} / \mathrm{kg})$ or left untreated. Five days p.i. spleen cells were cultured in the presence or absence of ConA $(3 \mu \mathrm{g} / \mathrm{ml})$ or MOG $(27 \mu \mathrm{g} / \mathrm{ml})$ for 48 or 72 hour respectively. Supernatant nitrate levels were measured from (a) spleen and (b) LN cultures using the Griess assay. Shown are means and SEM of values from individual mice $(n=5-9$ per group) from two separate experiments. There were no significant differences, by one-way ANOVA. 

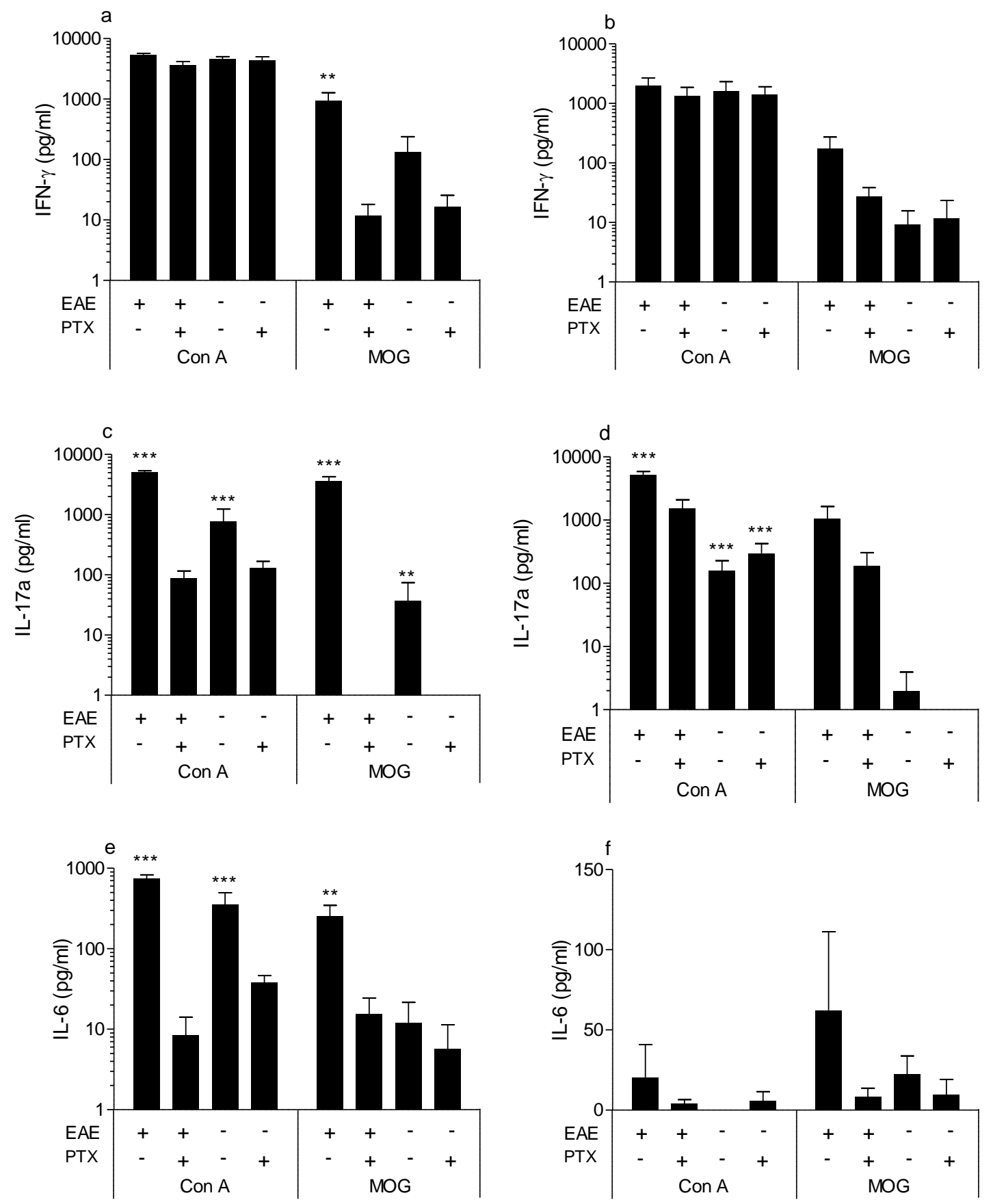

Figure 4.4 Ex vivo cytokine production is not altered in spleen and lymph node cells immediately after paclitaxel treatment (d0-4p.i)

C57BL/6 mice were immunised for EAE and treated with paclitaxel $(20 \mathrm{mg} / \mathrm{kg})$ or left untreated. Five days p.i. spleen (left column) or dLN (right column) cells were cultured in the presence or absence of Con A (3 $\mu \mathrm{g} / \mathrm{ml})$ or MOG $(27 \mu \mathrm{g} / \mathrm{ml})$ for 48 or 72 hour respectively. Cytokine production in the supernatants was assessed using a cytokine bead assay (a,b) IFN- $\gamma,(\mathrm{c}, \mathrm{d}) \mathrm{IL}-17 \mathrm{a}$, and (e,f) IL-6. One value in figure (b) within MOG-stimulated immunised untreated group was not included in the figure as it was far higher than the equivalent Con A stimulated sample, or any other sample; it was also identified as an outlier by the Grubbs test. No significant difference occurred when data was analysed either with or without this value. Shown are means and SEM of values from individual mice $(n=5-9$ per group) from two separate experiments $* * p$ $<0.01$ or $* *$ p $<0.001$ compared to immunised paclitaxel mice, by one-way ANOVA with Newman-Keuls multiple comparison test. 


\subsubsection{Immune cell infiltration into the spinal cord is inhibited following d0-4 or d6-12 p.i. paclitaxel treatment}

To explore if a similar alteration in immune response occurs in mice with a pre-established encephalgenic immune response, PTX was administered QOD on d6-12 p.i. and a similar series of immunological assessments were conducted on d13 p.i. At this time point, disease symptoms (i.e. ascending paralysis) were observed in immunised, untreated mice while PTX-treated mice remained disease free (Figure 4.5). Because cellular infiltration into the CNS is evident at this point in untreated, immunised mice, changes in cellularity within the spinal cord (SC) could be compared in immunised PTX and iUT mice. Using flow cytometry, a substantial population of $\mathrm{CD}^{+} \mathrm{T}$ cells was identified within the SC of iUT mice as assessed by percentage of total $\mathrm{CD} 45^{+}$cells or relative cell number (indicated by number of $\mathrm{CD}^{+}$events) (Figure 4.6a and b). In contrast to iUT mice, no increase in $\mathrm{CD}^{+}$populations were detected in the spinal cords of PTX d0-4 p.i. (iPTX $\mathrm{d}_{\mathrm{d}-4}$ ) or PTX d612p.i. QOD (iPTX $\mathrm{d6}_{-12}$ ) treated mice. To assess the effects of PTX treatment on myeloid populations (i.e. monocytes, macrophages, neutrophils), CD11b was used to collectively identify these populations. While the percentage of $\mathrm{CD} 11 \mathrm{~b}^{+}$cells within the SC of iUT mice did not significantly increase relative to other groups, the total number of cells did increase significantly, and this increase was only observed in the iUT mice (Figure 4.6c and d). These results indicate that by d13p.i., expansion or recruitment of $\mathrm{CD}^{+}$and $\mathrm{CD}_{11 \mathrm{~b}^{+}}$populations in the SC of iUT mice has occurred and treatment of mice with PTX completely prevents this expansion or recruitment.

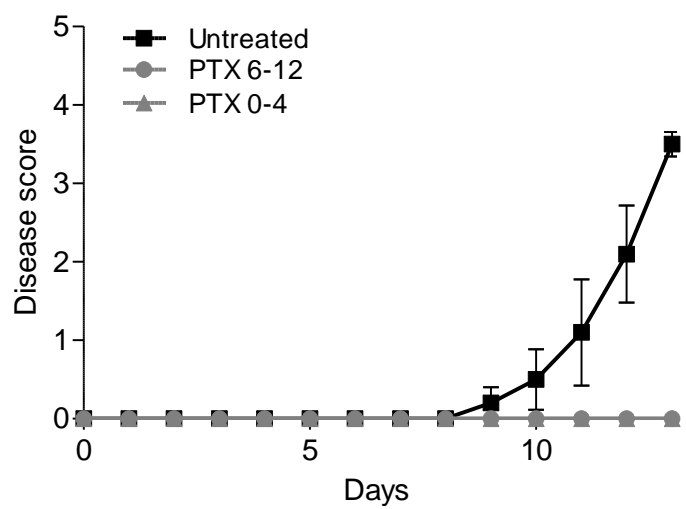

Figure 4.5 Paclitaxel treated mice do not exhibit disease symptoms 13 days after immunisation C57BL/6 mice were immunised for EAE and treated with PTX $(20 \mathrm{mg} / \mathrm{kg})$ on days $0-4$ p.i. or day 612 QOD, or left untreated. ( $\mathrm{n}=5$ /group) 
a
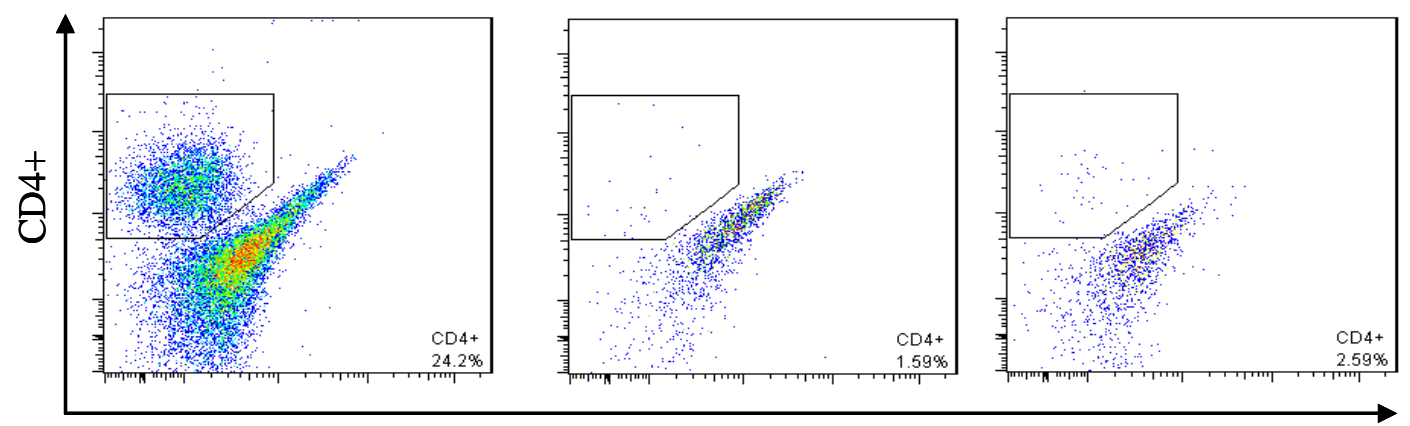

CD45+
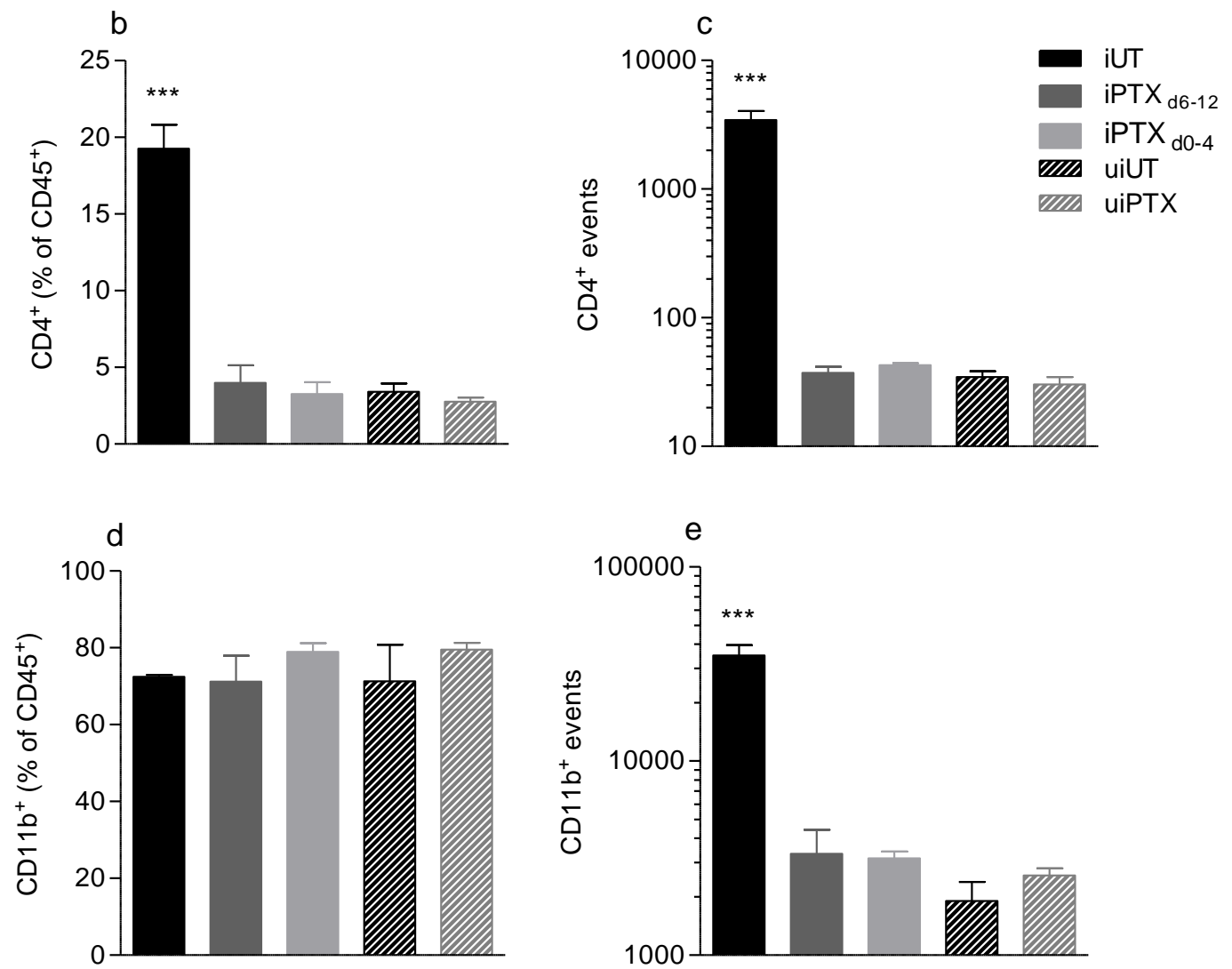

Figure 4.6 Paclitaxel inhibits immune cell infiltration into the CNS

C57BL/6 mice were immunised for EAE. Thirteen days p.i. spinal cord cells were isolated, stained for cell surface markers and analysed by flow cytometry. (a) Representative scatter plots of (from left to right) immunised untreated, immunised PTX d6-12 p.i. and unimmunised untreated $\mathrm{CD} 4^{+}$cells. (see appendix 1 for gating scheme). (b) Percent $\mathrm{CD}^{+}$positive cells of total $\mathrm{CD}_{4} 5^{+}$cells or (c) total number of $\mathrm{CD} 4^{+}$ events. (d) Percent $\mathrm{CD} 11 \mathrm{~b}^{+}$positive cells of total $\mathrm{CD} 45^{+}$cells or (e) total number of $\mathrm{CD} 11 \mathrm{~b}^{+}$events. Data represents one of two experiments $(n=5 /$ group, PTX 0-4 $n=3)$. * $p<0.05$ or **p $<0.01$ compared to immunised paclitaxel mice, by one-way ANOVA with Newman-Keuls multiple comparison test. 


\subsubsection{Administration of paclitaxel on d0-4p.i. has a different effect on spleen and dLN cellularity to paclitaxel administered on d6-12 p.i.}

Thirteen days after immunisation, mice treated with PTX on d6-12 p.i. QOD had similar total number of both splenocytes and LN cells compared to immunised, untreated mice (Figure 4.7f). Interestingly, iPTX $_{\mathrm{d} 0-4}$ mice had significantly higher number of splenocytes compared to iUT or iPTX $\mathrm{d} 6-12_{12}$ treated mice. To understand which cellular populations were affected by PTX treatment, the major cell populations were identified by flow cytometry. It was found that, within the spleens but not $\mathrm{dLN}_{\text {of }} \mathrm{iPTX}_{\mathrm{d} 0-4}$ mice, both $\mathrm{CD}^{+}$and $\mathrm{CD} 8^{+} \mathrm{T}$ cell numbers were lower compared to other immunised groups (Figure 4.7a-c). Although the reduction did not reach statistical significance, the difference in cell numbers was substantial ( $>2$ fold reduction). It is likely the reason the difference did not reach significance is due to the low numbers of mice in the iPTX $\mathrm{d} 0-4_{4}$ treatment group and inherent mouse-to-mouse variability. In contrast to T cells, a significant increase in F4/80 and $\mathrm{Gr}-1^{+}$cell numbers was observed in the $\mathrm{PPTX}_{\mathrm{d} 0-4}$ mice indicating that PTX does not cause lasting inhibitory effect on these populations (Figure 4.7e and f). Mice from the iPTX $_{\mathrm{d} 6-12}$ treatment group had similar numbers of $\mathrm{CD}^{+} \mathrm{CD}^{+}$and $\mathrm{F} 4 / 80^{+}$cells to that of iUT mice within their spleens and dLNs (Figure 4.7a-e), suggesting that proliferation and expansion of these cell populations occurred before PTX administration. The only significant difference between iUT and $\mathrm{iPTX}_{\mathrm{d} 6-12}$ mice was in $\mathrm{Gr}-1^{+}$cell numbers, which were reduced in the spleens of $\mathrm{PPTX}_{\mathrm{d} 6-12}$ mice (Figure 4.7e). As Gr-1 ${ }^{+}$cells, which are primarily neutrophils, have a rapid turnover, the reduction in this population in $\mathrm{PTX}_{\mathrm{d} 6-12}$ treated mice may indicate that PTX is inhibiting the replenishment of the neutrophil population. 

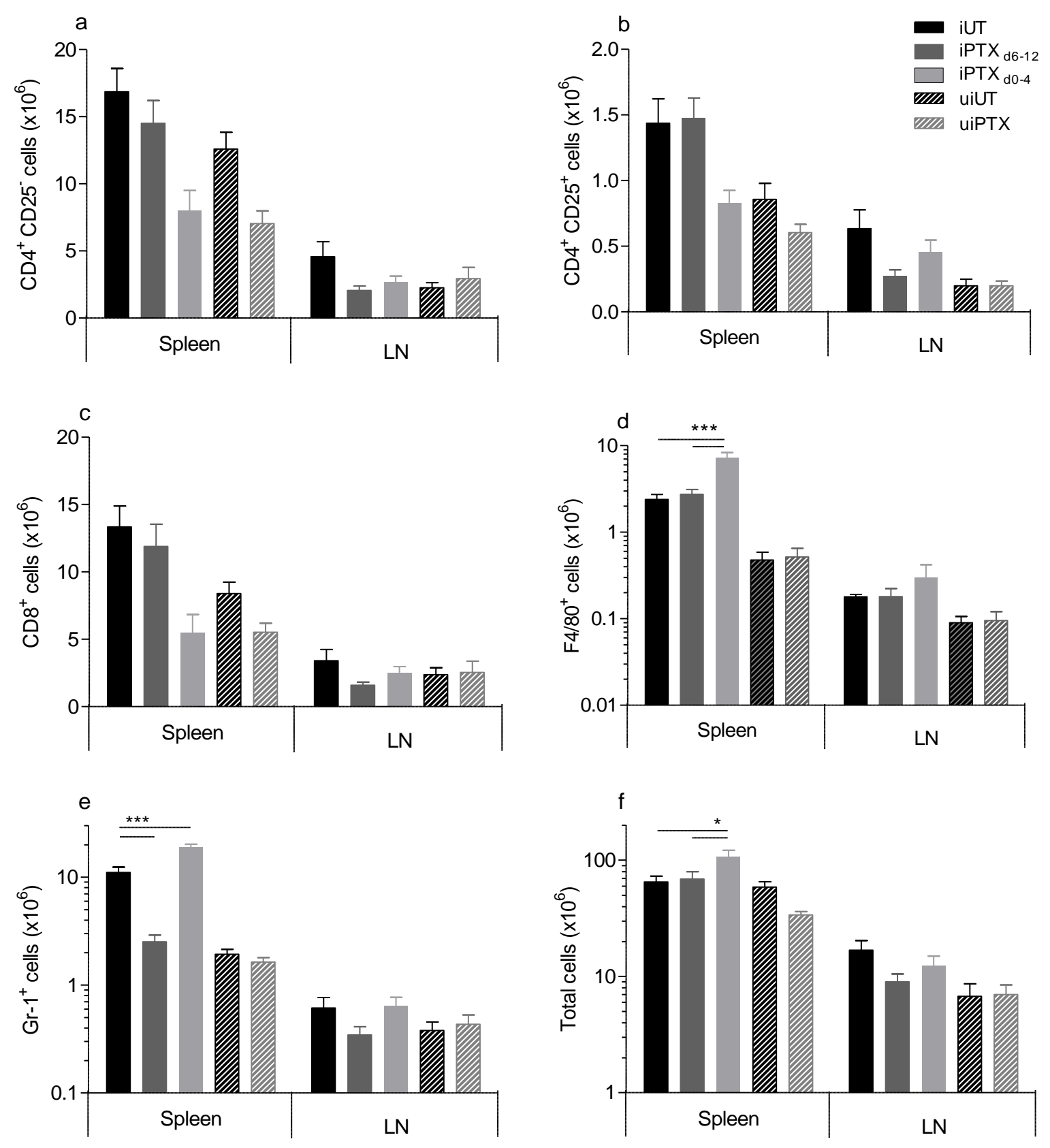

Figure 4.7 Paclitaxel alters spleen cellularity when administered on d0-4 p.i. or on d6-12 p.i.

C57BL/6 mice were immunised for EAE. Thirteen days p.i. spleen and dLN cells were isolated, counted, stained for cell surface markers and analysed by flow cytometry. Mean total cell numbers of (a) $\mathrm{CD} 4^{+} \mathrm{CD} 25^{-}$(b) $\mathrm{CD}^{+} \mathrm{CD} 25^{+}$(c) $\mathrm{CD}^{+}$(d) $\mathrm{F} 4 / 80^{+}$(e) $\mathrm{Gr}-1^{+}$and (f) total splenocytes and lymphocytes cells. Shown are means and SEM of values from individual mice from one (immunised PTX d0-4, unimmunised untreated and unimmunised PTX d6-12, $\mathrm{n}=3$-5/group) or two separate experiments (immuniszed PTX d6-12 and immunised untreated, $\mathrm{n}=10$ /group). $* \mathrm{p}<0.05$ or $* * * \mathrm{p}<0.001$ by one-way ANOVA with Newman-Keuls multiple comparison test. 


\subsubsection{Antigen-specific responses in spleen and $L N$ cells are unaffected following d6-12 p.i. paclitaxel treatment}

To determine if d6-12 p.i. PTX treatment alters cellular immune responses in addition to cellularity of lymphoid organs during EAE, the functional responses (i.e. proliferation and cytokine production) of spleen and LN cell were assessed on d13p.i.. When polyclonal proliferation was measured, reduced proliferation was observed within all immunised groups compared to unimmunised groups; however, no significant difference in splenocyte polyclonal proliferative responses were observed between drug-treated or untreated mice (Figure 4.8a). A similar effect was also observed within LN cell cultures (Figure 4.8b). Interestingly, the antigen-specific response in splenocytes from all immunised groups was low. However, while little antigen-specific proliferation was detected in iUT or iPTX $\mathrm{d} 0-4_{4}$ treated mice, antigen-specific proliferation was significantly higher in the PTX $_{\mathrm{d} 6-12}$ treated mice (Figure 4.8a). In contrast to splenocytes, no difference in antigen-specific proliferation was observed in cultures of LN cells from any of the immunised treatment group (Figure 4.8b). Previous studies have shown that enhanced production of NO can inhibit proliferation (Albina et al., 1991), yet there was no correlation between proliferation and NO production, suggesting that antigen-specific NO production was not preventing $\mathrm{T}$ cell proliferation in these cultures (Figure 4.9).

Although antigen-specific proliferation by splenocytes from all immunised groups was low, substantial inflammatory cytokine production by these cells was found within the cultured supernatants. IFN- $\gamma$ production by splenocytes from immunised mice was similar in MOG and Con A stimulated cultures, and no difference in IFN- $\gamma$ production between immunised PTX treated or untreated mice was detected (Figure 4.10a). Similarly, no difference was observed in IL-17 or IL-6 production by splenocytes from any of the immunised groups following MOG stimulation, although Con A stimulated splenocytes from $\mathrm{iPTX}_{\mathrm{d} 6-12}$ mice produced significantly less IL-17 but not IL-6 relative to the other immunised groups (Figure 4.10c and e). These data indicate that there is no significant difference in the antigen-specific IFN- $\gamma$, IL-17, and IL-6 responses of splenocytes from any of the immunised groups. 
In contrast to splenocyte responses, MOG stimulation of $\mathrm{LN}$ cells resulted in much lower levels of IFN- $\gamma$, although no difference in levels occurred between groups. After Con A stimulation, LN cells from immunised, $\mathrm{iPTX}_{\mathrm{d} 0-4}$ treated mice produced higher levels of IFN- $\gamma$ relative to iUT or $\mathrm{iPTX}_{\mathrm{d} 6-12}$ treated mice (Figure 4.10b). Likewise, no significant difference was detected in LN cells IL-17 responses between immunised groups following MOG stimulation while LN cells from $\mathrm{iPTX}_{\mathrm{d} 0-4}$ treated mice produced significantly higher levels of IL-17 following Con A stimulation compared to the other immunised groups (Figure 4.10d). Finally, only very low levels of IL-6 were detected in the LN cultures, and no significant differences were found between immunised groups. Together with the results from the splenocyte cytokine responses, these findings indicate that there is little difference in antigen-specific cytokine responses between the immunised groups 13 days p.i.. 

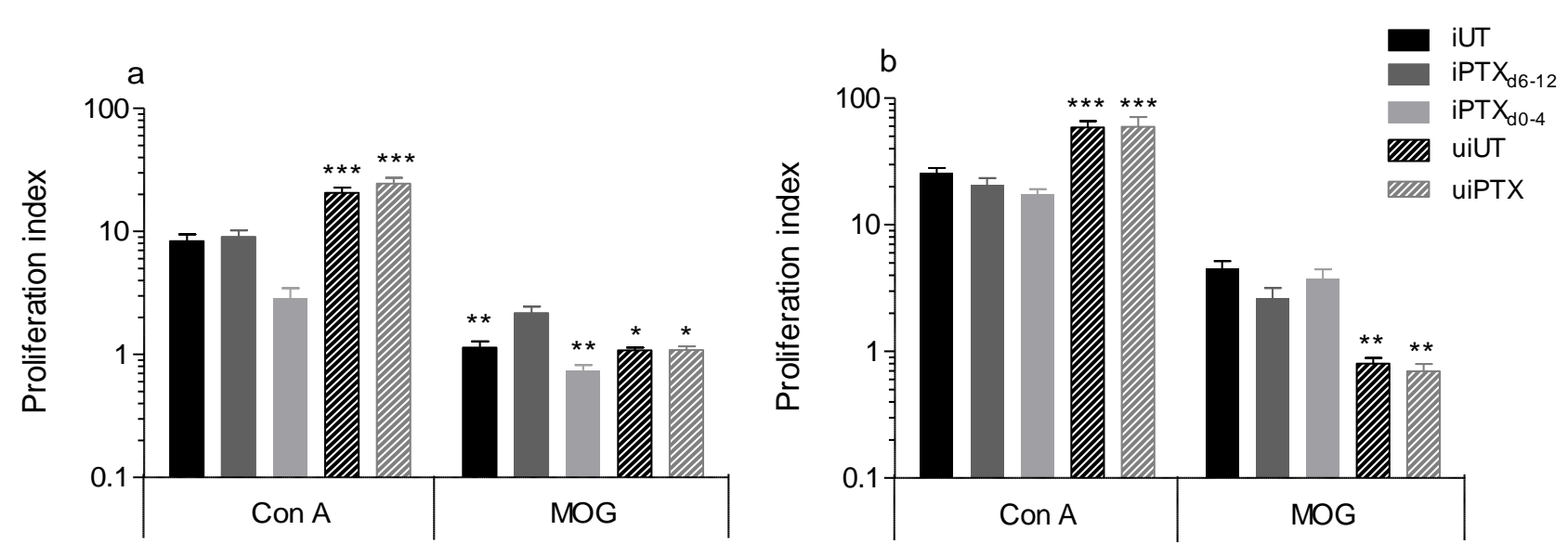

Figure 4.8 Paclitaxel does not inhibit ex vivo antigen-specific proliferation when administered on d6-12 p.i. C57BL/6 mice were immunised for EAE. Thirteen days p.i. (a) spleen and (b) lymph node cells were cultured in the presence or absence of Con A (3 $\mu \mathrm{g} / \mathrm{ml})$ or $\mathrm{MOG}(27 \mu \mathrm{g} / \mathrm{ml})$ for 48 or 72 hour respectively. Proliferation was assessed by ${ }^{3}[\mathrm{H}]$ thymidine $(1 \mu \mathrm{Ci})$ incorporation and is expressed as a proliferation index; CPM of stimulated wells/CPM of media alone. Shown are means and SEM of values from individual mice from one (spleen; immunised PTX d0-4, unimmunised untreated and unimmunised PTX d6-12, n= 3-5/group) or two separate experiments (immunised PTX d6-12 and immunised untreated, $\mathrm{n}=10$ /group). ${ }^{*} \mathrm{p}<0.05, * * \mathrm{p}<0.01$ or $* * * \mathrm{p}<$ 0.001 compared to immunised paclitaxel mice (d6-12 p.i.), by one-way ANOVA with Newman-Keuls multiple comparison test.
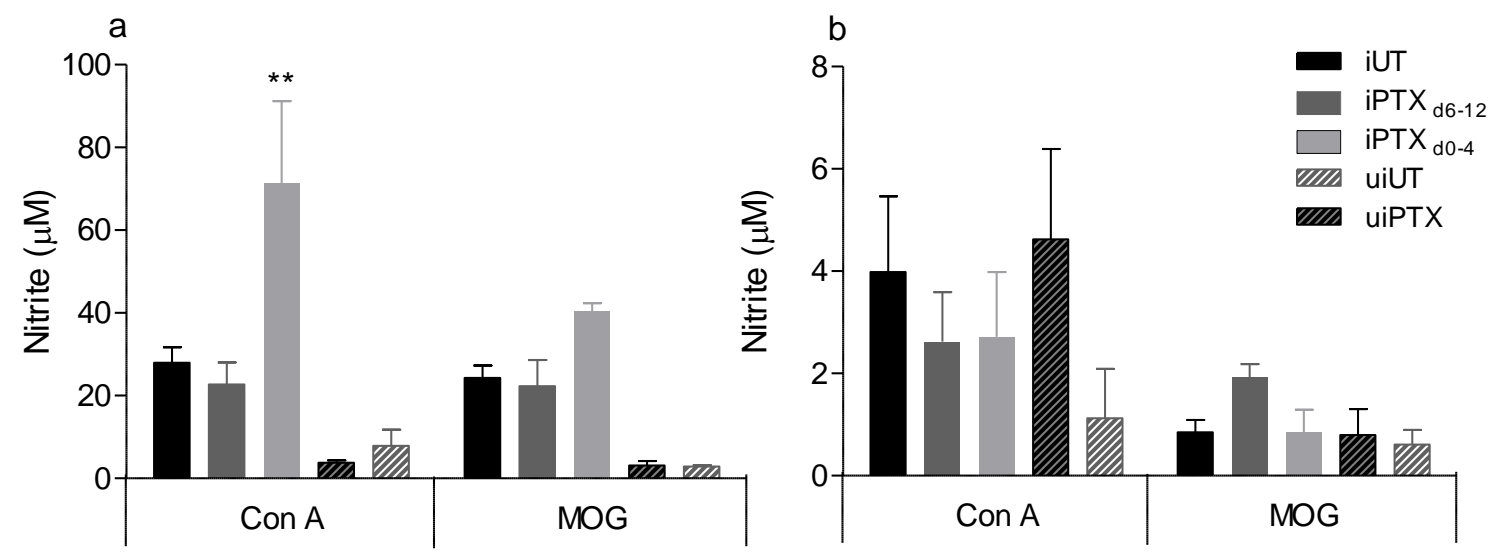

Figure 4.9 Antigen-specific nitric oxide production is not altered in ex vivo spleen and lymph node cells from d6-12 p.i. QOD paclitaxel treated mice relative to untreated mice

C57BL/6 mice were immunised for EAE. Thirteen days p.i. spleen cells were cultured in the presence or absence of Con A $(3 \mu \mathrm{g} / \mathrm{ml})$ or MOG $(27 \mu \mathrm{g} / \mathrm{ml})$ for 48 or 72 hour respectively. Supernatant nitrate levels were measured from (a) spleen and (b) LN cultures using the griess assay. Shown are means and SEM of values from individual mice from one (spleen; immunised PTX d0-4, unimmunised untreated and unimmunised PTX d6-12, $\mathrm{n}=3-5$ /group) or two separate experiments (immunised PTX d6-12 and immunised untreated, $\mathrm{n}=10$ /group). ${ }^{* *} \mathrm{p}<0.01$ compared to immunised paclitaxel mice (d6-12 p.i.), by one-way ANOVA with Newman-Keuls multiple comparison test. 

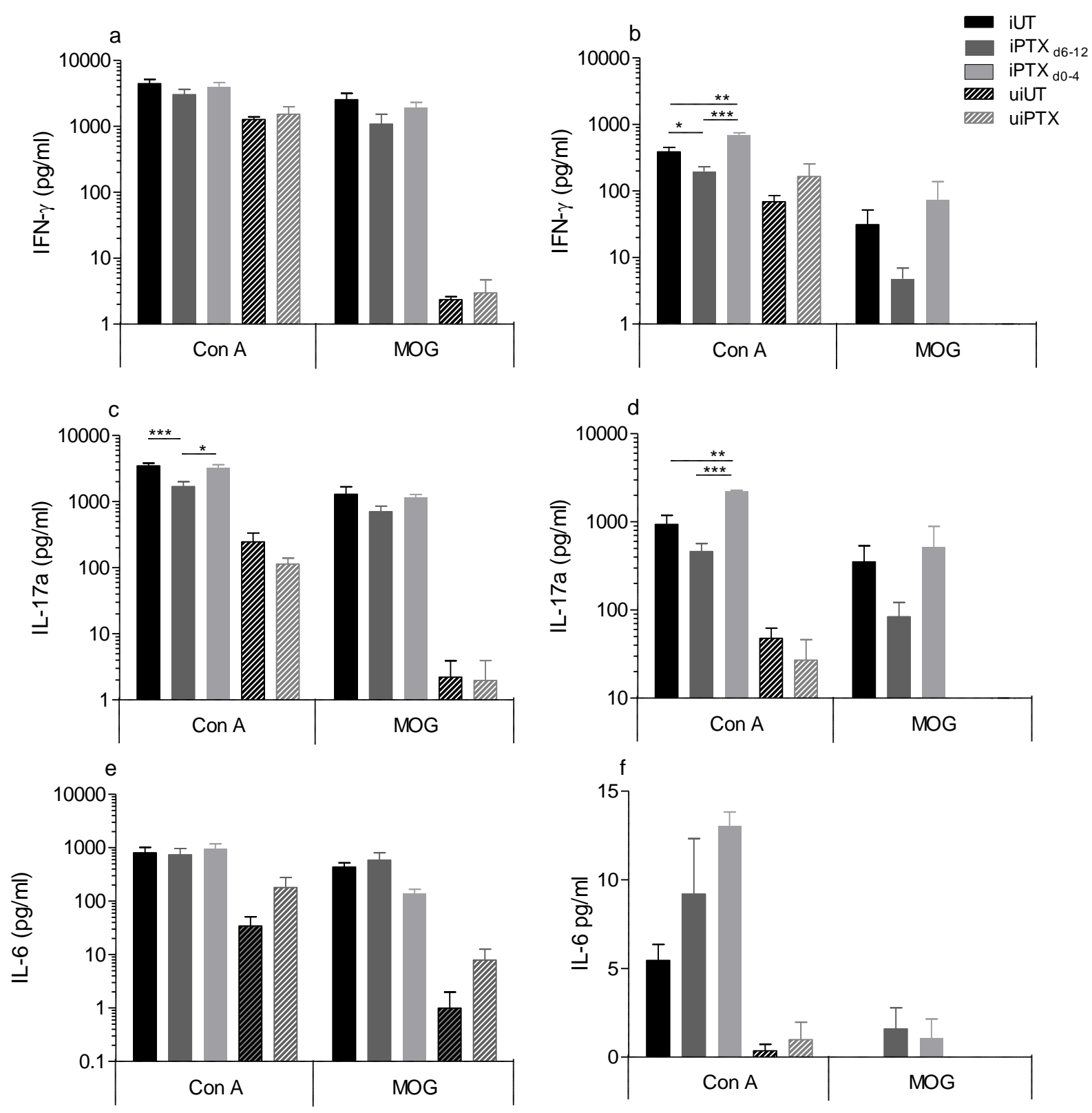

Figure 4.10 Antigen-specific cytokine production is not altered in ex vivo spleen and lymph node cells from paclitaxel treated mice

C57BL/6 mice were immunised for EAE. Thirteen days p.i. spleen (left column) or dLN (right column) cells were cultured in the presence or absence of ConA $(3 \mu \mathrm{g} / \mathrm{ml})$ or MOG $(27 \mu \mathrm{g} / \mathrm{ml})$ for 48 or 72 hour respectively. Cytokine production in the supernatants was assessed using a cytokine bead assay (a,b) IFN- $\gamma$, (c,d) IL-17a, and (e,f) IL-6. Shown are means and SEM of values from individual mice from one (LN, n= 3-5 mice/group; spleen immunised PTX d0-4, unimmunised untreated and unimmunised PTX d6-12, n=3-5/group) or two separate experiments (spleen, immunised PTX d6-12 and immunised untreated, $\mathrm{n}=10$ /group). *p < $0.05, * * \mathrm{p}<0.01$ or $* * * \mathrm{p}<0.001$ compared to immunised paclitaxel mice (d6-12 p.i.), by one-way ANOVA with Newman-Keuls multiple comparison test. 


\subsubsection{Paclitaxel inhibits in vitro antigen-specific proliferation}

A previous study conducted in our laboratory had found in vivo proliferation of MOG specific $\mathrm{T}$ cells within the dLN was not inhibited by 5 daily doses of PTX in EAE immunised animals (Crume, 2007; Crume et al., 2009). It was postulated that: 1) the drug concentrations in the dLNs were not high enough to induce mitotic arrest or 2) high PgP expression on the $\mathrm{T}$ cells allowed the cells to pump out PTX and resist mitotic arrest (Crume, 2007). Either of these explanations are conceivably correct, particularly given that antigen-specific responses were completely inhibited in the spleen but not the dLN of immunised mice treated with PTX on d0-4 p.i. However, the alterations in cellularity observed in the current study and, in particular, the inhibition of $\mathrm{CD}^{+}$and $\mathrm{Gr}-1^{+}$cell expansion, would suggest that PTX treatment did affect cellular processes occurring in the dLN. To better understand the specific effects of PTX on T cells in the lymph node or spleen, further experiments were conducted to confirm the anti-proliferative actions of PTX and its analogue DTX.

It has previously been shown using an MTT assay, that PTX inhibits splenocyte proliferation at low nanomolar concentrations following polyclonal stimulation in vitro (Crume, 2007). To exclude the possibility that antigen-specific proliferation was less prone to the anti-proliferative actions of PTX, in vitro proliferation assays were conducted using splenocytes from 2D2 mice, which have transgenic $\mathrm{T}$ cell receptors specific for the MOG peptide (Bettelli et al., 2003). To specifically assess the effects of PTX on proliferation over the duration of the culture period, splenocytes were labelled with carboxyfluorescein diacetate succinimidyl ester (CFSE) at the start of culture (Wilmes et al., 2010a). CFSE readily enters the cell where it is converted to a non-permeable and fluorescent form; thus the cells are permanently labeled. Subsequent proliferation of CFSE-labelled cells leads to a reduction of fluorescence in the daughter cells, and this reduction can be quantified via flow cytometry (Parish et al., 2009). This method has two distinct advantages over the MTT assay: 1) the MTT assay uses metabolic activity as an indicator of proliferation since increased cell numbers results increase reduction of the MTT dye (Berridge et al., 1993); while, the CFSE-based assay measures proliferation directly and 2) by using flow cytometry, proliferation of specific cell populations can be identified with the CFSE assay. 
In accordance with previous findings that used polyclonal simulation (Brown et al., 1985; Crume, 2007), the current study found that both PTX and DTX inhibited antigen-specific proliferation of splenocytes at low nanomolar concentrations (Figure 4.11a and b). Additionally, inhibition of proliferation was found to occur in all splenocytes as well as $\mathrm{CD}^{+} \mathrm{T}$ cells (Figure 4.11a and b). A similar anti-proliferative effect was also observed when splenocytes from immunised mice were isolated and stimulated in vitro in the presence of PTX or DTX (Figure 4.12). Both PTX and DTX had strong inhibitory effects on proliferation in response to either polyclonal or antigen-specific stimulation (Figure 4.12c and d). Collectively these experiments confirm that within the context of autoimmune proliferation, MSDs can effectively inhibit proliferation at nanomolar concentrations in vitro.
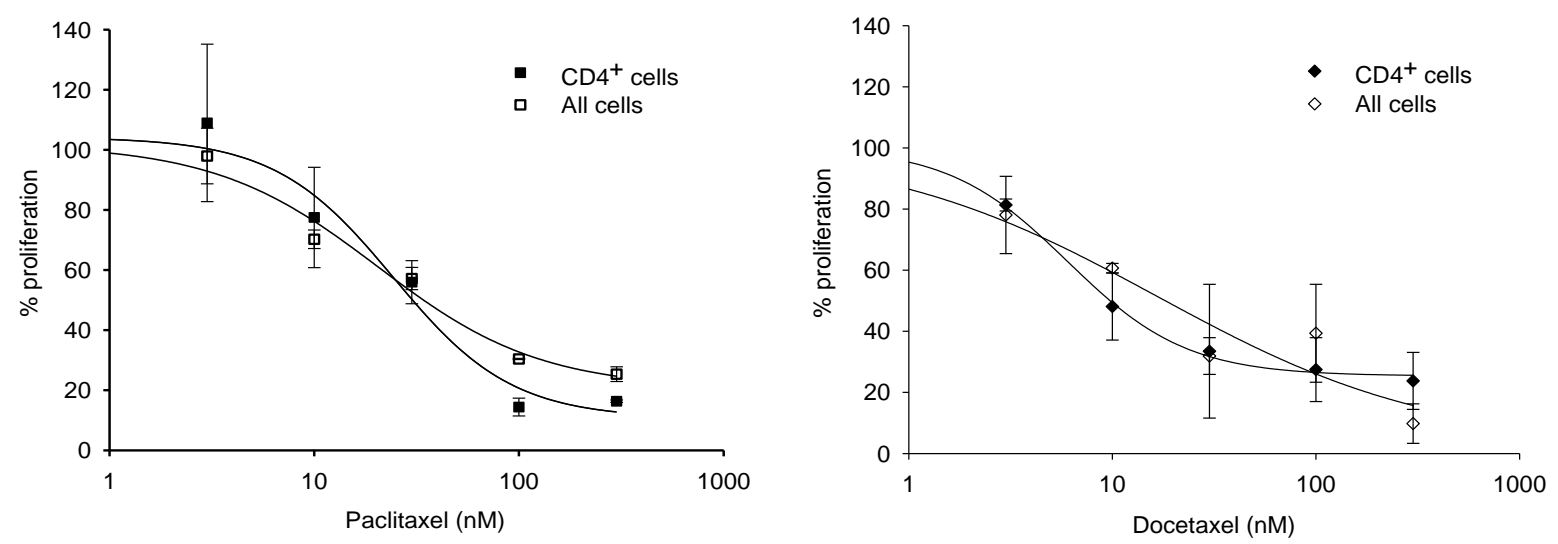

Figure 4.11. In vitro paclitaxel inhibits antigen-specific proliferation

Splenocytes from 2D2 mice were pulsed with CFSE and cultured in the presence of MOG $(27 \mu \mathrm{g} / \mathrm{ml})$ and indicated concentration of (a) paclitaxel or (b) docetaxel. After 72 hours, cells were stained for cell surface markers and proliferation assessed by flow cytometry. The results are expressed as percent proliferation of all splenocytes or $\mathrm{CD}^{+}$splenocytes relative to proliferating untreated cells. Results represent means and SEM of two separate experiments conducted in duplicate. 

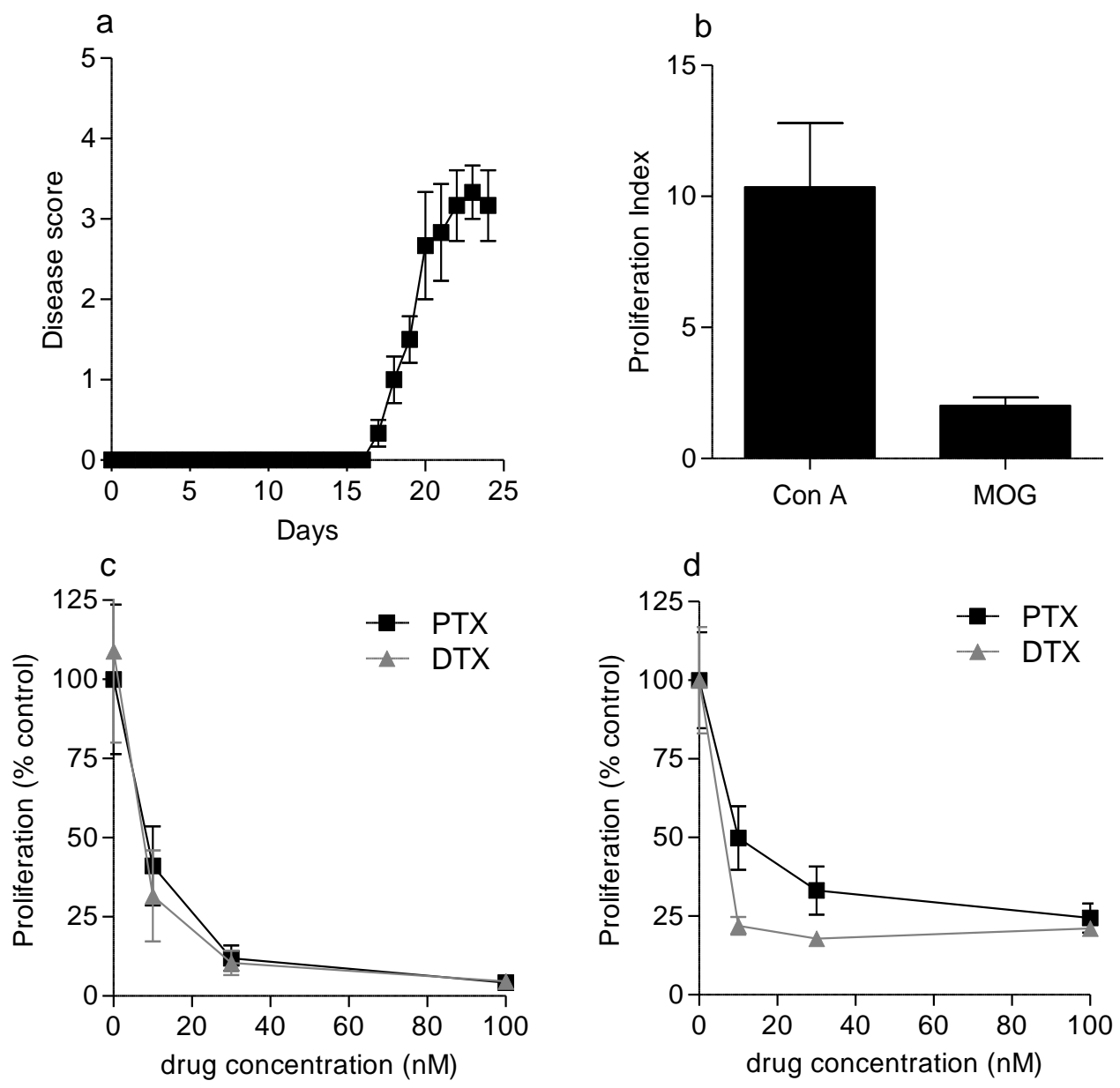

Figure 4.12. In vitro paclitaxel and docetaxel inhibit proliferation in splenocytes isolated from EAE immunised mice

C57BL/6 mice were immunised for EAE. Splenocytes were isolated 24 days p.i., pulsed with CFSE and cultured in the presence or absence of indicated concentration of paclitaxel or docetaxel and Con A ( $3 \mu \mathrm{g} / \mathrm{ml})$ or MOG35-55 for 48 or 72 hours respectively. (a) disease score, (b) proliferation of Con A $(3 \mu \mathrm{g} / \mathrm{ml})$ or MOG $(27 \mu \mathrm{g} / \mathrm{ml})$ stimulated non-drug treated cells represented as proliferation index; expressed as CPM of stimulated wells/CPM of media alone. (c) Con A induced proliferation; expressed as percent of non-drug treated cells (d) MOG induced proliferation; expressed as percent of non-drug treated cells. Proliferation was assessed by flow cytometry. Shown are means and SEM of individual mice $(n=5)$. 


\subsubsection{Paclitaxel inhibits in vivo antigen-specific proliferation}

To determine if PTX altered in vivo antigen-specific proliferation within the dLN of EAE immunised mice, within our experimental system, a similar experiment to that described by Crume et al. was conducted (Crume et al., 2007; Crume et al., 2009). However in contrast to Crume et al., the proliferation of antigen-specific T cells in the spleen and blood was also analysed to identify if there was a divergent effect of PTX on proliferation between these organs. Additionally, the experimental design was altered to more accurately distinguish the transferred antigen-specific $\mathrm{T}$ cells from the resident $\mathrm{T}$ cells within the recipient mice. Specifically, in the original study CFSE labelled 2D2 lymphocytes were adoptively transferred to WT C57BL/6 recipient mice and proliferation of these transferred cells was measured by a reduction in CFSE fluorescence; however, CFSE was also used to distinguish the transferred $\mathrm{T}$ cells from those of the recipient mice (Crume, 2007). As proliferation causes a reduction in CFSE florescence, it was possible that multiple cycles of proliferation would have resulted in the transferred $\mathrm{T}$ cells becoming indistinguishable from the recipient $\mathrm{T}$ cells when identified by fluorescence alone. To avoid this possibility in the current study, the 2D2 cells (which express the CD45.2 isoform) were adoptively transferred into congenic C57BL/6 (B6.SJL-ptprca) mice, which express CD45.1 on a C57BL/6 background. This approach allowed the adoptively transferred cells to be independently identified by CD45.2 expression, removing the reliance on CFSE florescence as a way to differentiate transferred cells from recipient cells.

In contrast to the previous studies findings, PTX treatment resulted in a significant reduction in the percentage of $\mathrm{CD}^{+} 2 \mathrm{D} 2$ cells within the $\mathrm{dLN}$ that proliferated following EAE immunisation (Figure 4.13b). Interestingly while the percent of 2D2 CD4 ${ }^{+}$ proliferation was reduced in iPTX treated mice, 2D2 proliferation was still significantly higher than in unimmunised mice, indicating that PTX attenuated but did not completely inhibit proliferation within the $\mathrm{dLN}$. However, when looking at the representative plots of iUT compared to iPTX treated, it appears that fewer CD4 ${ }^{+} 2 \mathrm{D} 2$ cells are present in iPTX treated mice (Figure 4.13a). To quantify this difference, the total number of 2D2 CD4 ${ }^{+}$ proliferative events was assessed. When comparing total $\mathrm{CD}^{+} 2 \mathrm{D} 2$ proliferative events between iPTX treated and iUT, mice it is clear that PTX has a dramatic effect on antigen- 
specific proliferation within the dLN. No significant difference was observed between proliferative events in iPTX treated and uiPTX mice; whereas, iUT mice exhibited significantly more $\mathrm{CD}^{+} 2 \mathrm{D} 2$ proliferation than both iPTX and unimmunised groups (Figure 4.13c). Additionally, when the numbers of $2 \mathrm{D} 2 \mathrm{CD}^{+}$proliferative and nonproliferative events were separated by their cycles of proliferation (i.e. generations of cell division), it can be seen that a substantial proportion of the total iUT events had undergone 5-7 generations of cell division (Figure 4.13d). In contrast, only very small populations of iPTX treated mice had undergone such divisions (Figure 4.13d). Moreover, no difference in the numbers of non-proliferating $\mathrm{CD} 4^{+} 2 \mathrm{D} 2$ events was detected between iUT and iPTX treated mice (data not shown). Collectively, these findings indicate that PTX was inhibiting or inducing death in the proliferating population; whereas, the non-proliferating population remained viable.

Analysis of the transferred $\mathrm{CD} 4^{+} 2 \mathrm{D} 2$ cells in the spleen or blood revealed that there was a high level of antigen-specific proliferation 5 days post immunisation. The anti-proliferative actions of PTX were even more pronounced in these tissues compared to the dLNs, as PTX treatment completely inhibited antigen-specific proliferation (Figure 4.14a-d). This inhibition of proliferation is evident when comparing the percent of transferred cells that are proliferating as well as the total proliferative events. The differences in the magnitude of the effect between the spleen or blood and the dLN may indicate that PTX does not reach as high a concentration in the dLNs as in the other tissues sampled. Taken together, these experiments indicate that PTX in fact inhibits antigen-specific T cell proliferation in vivo. 

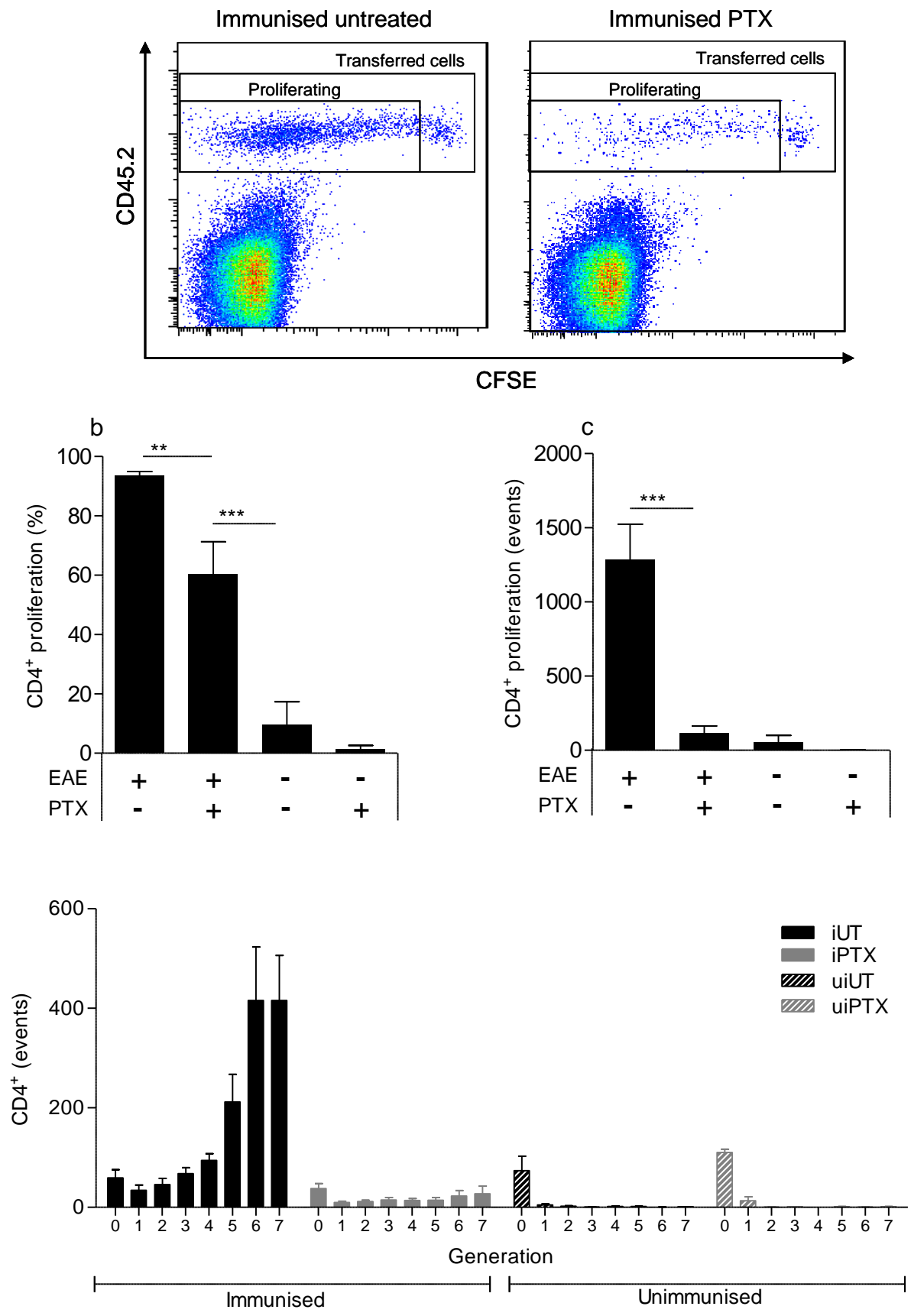

Figure 4.13 Paclitaxel attenuates in vivo antigen $\mathrm{T}$ cell proliferation within the lymph node

Spleen and LN cells from 2D2 mice were stained with CFSE and transferred to congenic C57BL/6 recipient mice (B6.SJL-ptprca). One day following transfer, mice were immunised for EAE or left unimmunised and either drug treated (PTX 20mg/kg, d0-4p.i.) or left untreated. Five days p.i., cells from the dLN were isolated, stained for cell surface markers and analysed by flow cytometry. All samples were harvested, prepared and stained in an identical manner. The same number of events were collected for each sample and identical gating and analysis of each sample was conducted. (a) representative plots of lymphocytes isolated from immunised untreated and immunised paclitaxel treated mice (each plot represent a concatenation of data from 3 individual mice). (b) $\mathrm{CD} 4^{+}$proliferation; percent of transferred $2 \mathrm{D} 2 \mathrm{CD}^{+}$cells isolated from the dLNs that had undergone proliferation in vivo (see appendix 2 for the gating scheme). (c) $\mathrm{CD} 4^{+}$proliferation; total collected events of $2 \mathrm{D} 2 \mathrm{CD}^{+}$cells isolated per sample collected from the dLNs that had undergone proliferation in vivo. (d) $\mathrm{CD} 4^{+}$proliferation as events per cell division; represented as total collected events of $2 \mathrm{D} 2 \mathrm{CD} 4^{+}$cells per generation of proliferation (i.e. generation $0=$ cell that has not undergone any proliferation, generation $7=$ cells that have undergone 7 cell divisions). Discrete generations were defined using FloJo 7.6.1 cell proliferation analysis software. Shown are means and SEM of values from individual mice from two separate experiments ( $n=7 /$ immunised groups, $n=4 /$ unimmunised groups). $* * \mathrm{p}<0.01$ or $* * * \mathrm{p}<0.001$ by one-way ANOVA with Newman-Keuls multiple comparison test. 

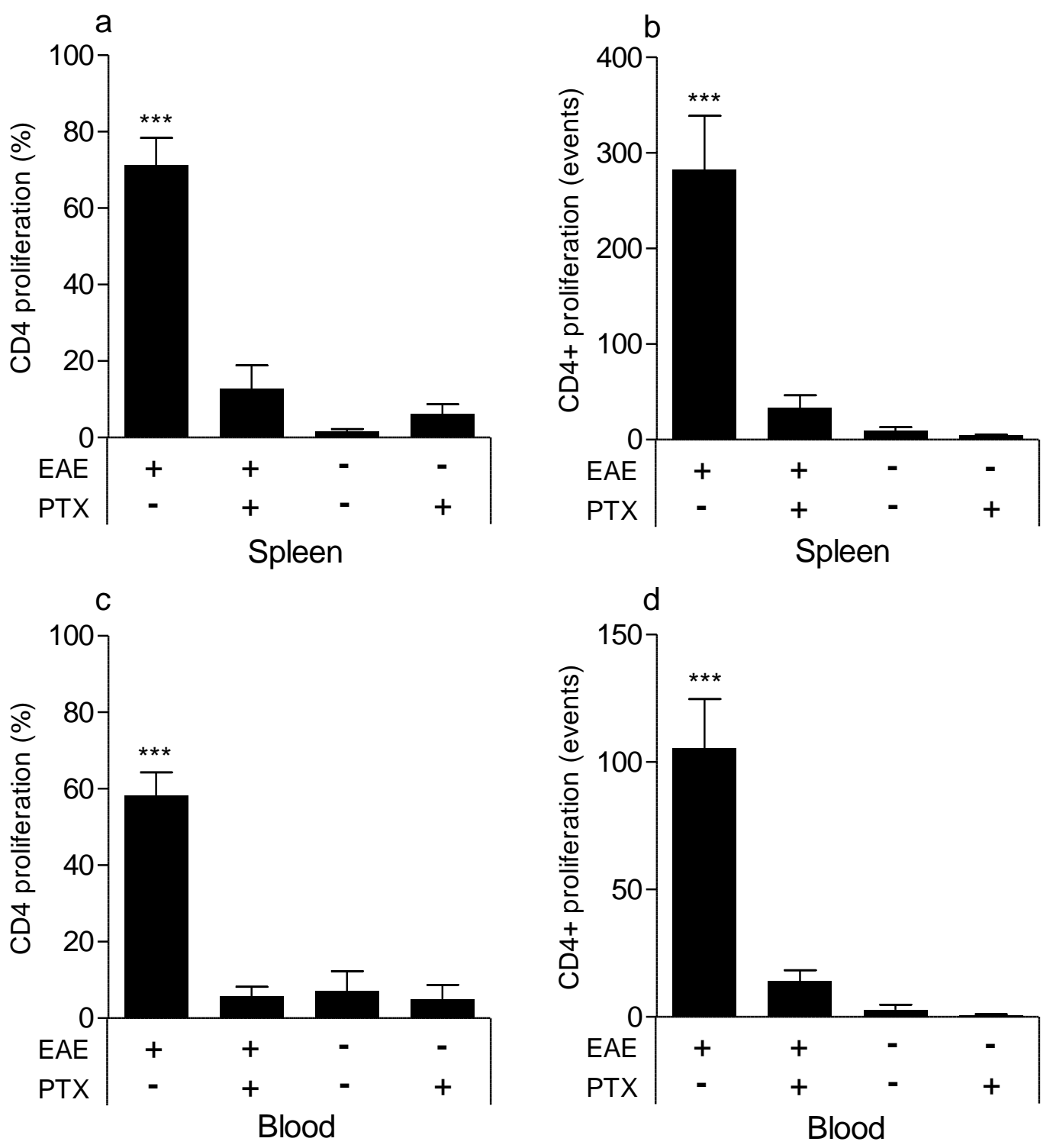

Figure 4.14 Paclitaxel inhibits in vivo antigen $T$ cell proliferation within the blood and spleen

Spleen and LN cells from 2D2 mice were stained with CFSE and transferred to congenic C57BL/6 recipient mice (B6.SJL-ptprca). One day following transfer, mice were immunised for EAE or left unimmunised and either drug treated (PTX 20mg/kg, d0-4p.i.) or left untreated. Five days p.i. cells from the spleen and blood were isolated stained for cell surface markers and analysed by flow cytometry. All samples were harvested, prepared and stained in an identical manner. The same number of events were collected for each sample and identical gating and analysis of each sample was conducted. (a,c) $\mathrm{CD} 4^{+}$proliferation; percent of transferred $2 \mathrm{D} 2 \mathrm{CD}^{+}$cells isolated from the (a) spleen or (c) blood that had undergone proliferation in vivo. (b,d) $\mathrm{CD} 4^{+}$ proliferation; total collected events of $2 \mathrm{D} 2 \mathrm{CD}^{+}$cells from individual samples isolated from the (b) spleen or (d) blood that had undergone proliferation in vivo. Shown are means and SEM of values from individual mice from two separate experiments ( $n=7 /$ immunised groups, $n=4 /$ unimmunised groups). ${ }^{* * *} p<0.001$ by one-way ANOVA with Newman-Keuls multiple comparison test. 


\subsubsection{Paclitaxel inhibits in vivo immune cell migration}

The inhibition of antigen-specific proliferation by PTX is likely to explain why expansion of $\mathrm{T}$ cell populations and induction of antigen-specific responses were inhibited following iPTX $_{\mathrm{d} 0-4}$ treatment. However, PTX also inhibited the increase in size of $\mathrm{F} 4 / 80^{+}$populations, and as these cells do not readily proliferate, PTX must be causing an alteration in the size of this population via another mechanism. A potential explanation for this is that trafficking of $\mathrm{F} 4 / 80^{+}$cells may have been altered. Additionally, despite similar antigenspecific responses in splenocytes from iPTX $\mathrm{d}_{6-12}$, increases in $\mathrm{CD}^{+}$and $\mathrm{CD}^{+} \mathrm{lb}^{+}$ populations within the SC were only observed in iUT mice. Collectively these results suggest that PTX could be inhibiting the migration of immune cells to secondary lymphoid organs as well as from the periphery into the CNS.

To explore the potential of PTX to inhibit immune cell migration in vivo without the confounding issue of proliferation that occurs following immunisation, the effect of PTX on chemokine-induced cell migration was investigated. To achieve this, the chemokine CCL5 (RANTES) was injected once into the left hind flanks of mice; whereas, an equal volume of PBS was injected into the right hind flank, and the migration of CFSE-labelled 2D2 cells to the right or left dLN was assessed. CFSE-labelled 2D2 lymphocytes were injected i.p., and one day later mice were either injected i.p., with PTX $(20 \mathrm{mg} / \mathrm{kg} / \mathrm{d})$ or an equal volume of PBS for five consecutive days. Following the final drug administration CCL5 (left hind flank) or PBS (right hind flank) was injected s.c., and 18 hours later, the dLNs were isolated and analysed by flow cytometry. Since CCL5 can attract a number of immune cells including $\mathrm{T}$ cells and macrophages (Schall et al., 1990), it was anticipated that cell numbers within the LN that drained the CCL5 injection site would increase. To control for potential variations in injection technique, some mice were injected with PBS in both the left and right hind flanks; no differences in cell numbers between LNs were observed in these mice (data not shown).

As expected, a significant increase in $\mathrm{CD}^{+}$cell numbers was observed in LNs draining the CCL5 injection site in untreated mice (Figure 4.15a). In contrast, no increase was detected in PTX-treated mice, indicating that PTX treatment inhibited CCL5-mediated CD4 ${ }^{+}$ 
migration into the dLN. Migration of $\mathrm{F} 4 / 80^{+}$cells was also inhibited by PTX treatment, as shown by the significant increase in $\mathrm{F} 4 / 80^{+}$cells within the $\mathrm{LN}$ draining the CCL5 injection site in untreated but not PTX-treated mice (Figure 4.15b). Furthermore, when total cell numbers were analysed, PTX treatment was found to have a general effect on inhibiting migration (Figure 4.15c). Collectively, these findings indicate that PTX inhibits chemokine-induced immune cell migration. However, when the numbers of $2 \mathrm{D} 2 \mathrm{CD} 4^{+} \mathrm{T}$ cells were assessed, the difference between the LNs that drain the CCL5 or PBS injection sites in either PTX treated or untreated mice did not reach significance (Figure 4.16). This finding may be due to the combined effects of individual mouse variation and the relatively small numbers of 2D2 cells detected in the dLN. Alternatively, it is possible that 2D2 $\mathrm{CD}^{+} \mathrm{T}$ cells express less CCL5 receptors (e.g. CCR5) than the WT recipient CD4 ${ }^{+} \mathrm{T}$ cells and therefore did not migrate as readily in response to the CCL5. 

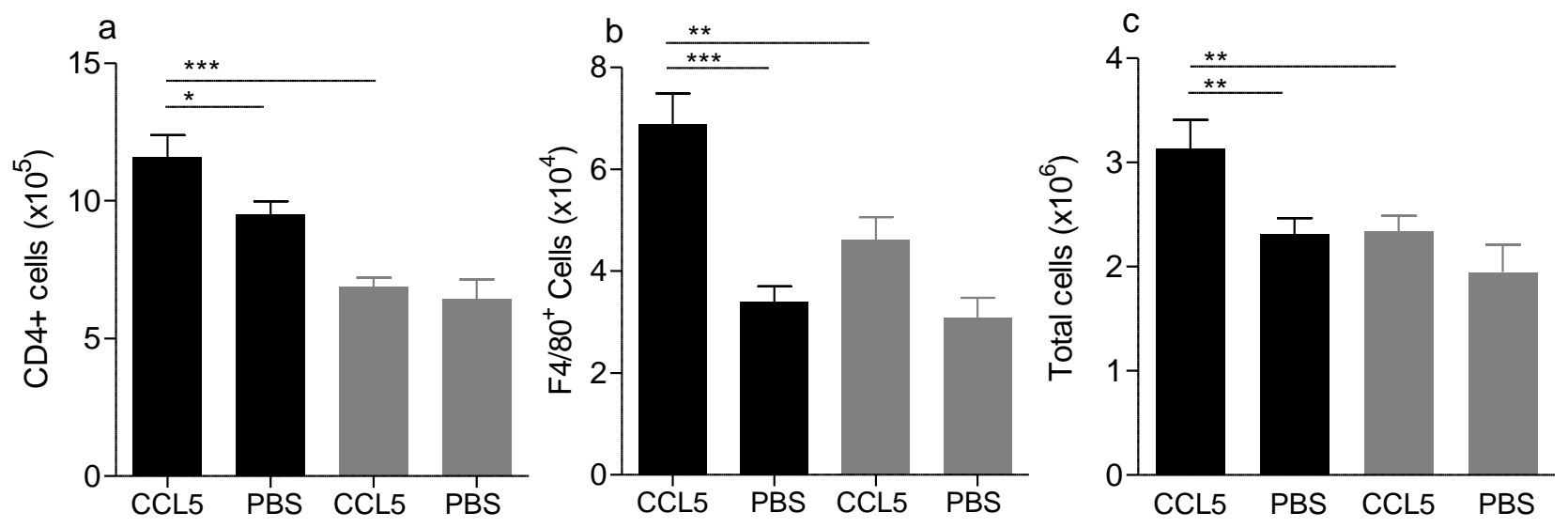

Figure 4.15 In vivo migration of $T$ cells and macrophages is inhibited by paclitaxel

Spleen and LN cells from 2D2 mice were stained with CFSE and transferred to C57BL/6 recipient mice. One day following transfer, mice were drug treated (PTX $20 \mathrm{mg} / \mathrm{kg} / \mathrm{d}$ for 5 consecutive days) or left untreated. Six days after

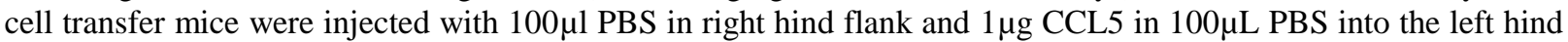
flank. 18 hours following hind flank injections, cells from the dLNs were isolated and counted. (a) $\mathrm{CD} 4^{+} \mathrm{T}$ cells, (b) $\mathrm{F} 4 / 80^{+}$cells, (c) total cells within individual dLN. Shown are means and SEM of values from individual mice from two separate experiments $(\mathrm{n}=12-13$ group). $* * \mathrm{p}<0.01$ by one-way ANOVA with Newman-Keuls multiple comparison test. "Untreated" mice received injections of the equivalent concentration of vehicle that would be present in drug treated mice.

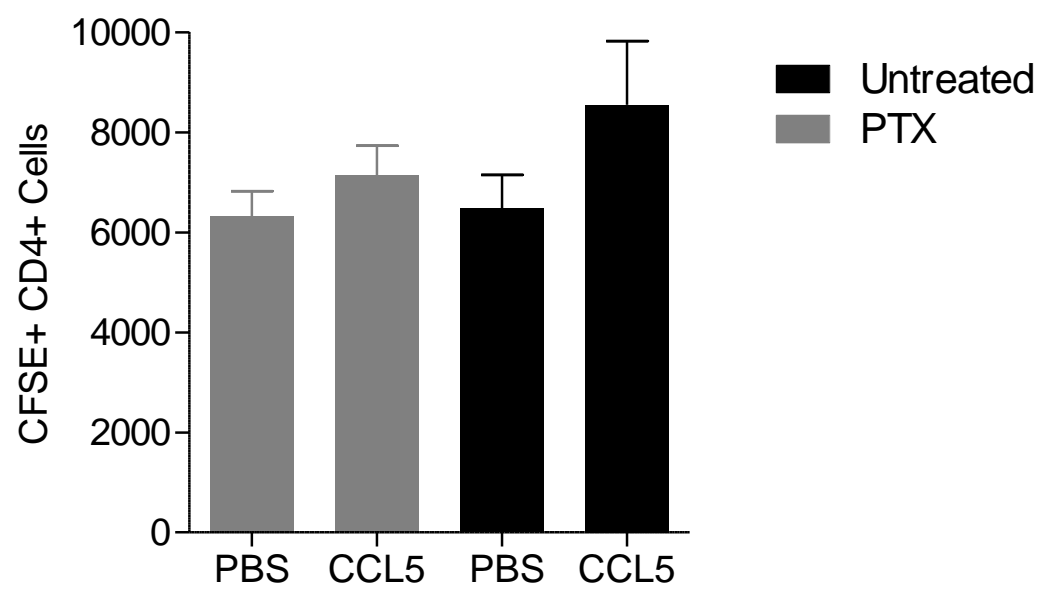

Figure 4.16 In vivo migration of adoptively transferred antigen-specific cells is not significantly altered, following paclitaxel treatment

Spleen and LN cells from 2D2 mice were stained with CFSE and transferred to C57BL/6 recipient mice. One day following transfer, mice were drug treated (PTX $20 \mathrm{mg} / \mathrm{kg} / \mathrm{d}$ for 5 consecutive days) or left untreated. Six days after cell transfer mice were injected with $100 \mu 1$ PBS in right hind flank and $100 \mu 1$ PBS or $1 \mu \mathrm{g}$ CCL5 in $100 \mu \mathrm{L}$ PBS into the left hind flank. 18 hours following hind flank injections cells from the dLNs were isolated and counted. Shown are means and SEM of values from individual mice from two separate experiments $(n=12-$ 13 group). No significant difference between groups by one-way ANOVA with Newman-Keuls multiple comparisons test. "Untreated" mice received injections of the equivalent concentration of vehicle that would be present in drug treated mice. 


\subsection{Discussion}

The experiments conducted in this chapter were focused on assessing potential changes in immune response immediately following PTX treatment and determining what effect drug treatment had on cellular proliferation and migration. In contrast to the previous chapter's findings, that PTX and other MSDs had little effect on long-term immune responses, the current chapter demonstrates that immediately after PTX administration, inflammatory disease responses are significantly altered. These drug induced changes appear to be associated with inhibition of immune cell proliferation and migration.

Trafficking of immune cells into and out of lymphoid organs, as well as migration from the periphery into the CNS, are crucial processes for the development of EAE. In the present study it was clearly demonstrated that PTX treatment inhibited both macrophage and T cell migration. The impact of inhibited migration within the context of EAE is substantial. Immune cell migration into the dLN is important in initial induction of an encephalogenic response. It is also vital for the immune cell infiltration into the CNS and subsequent development of disease symptoms (Ajami et al., 2011; Carlson et al., 2008). Inhibition of immune cell migration from the periphery into the CNS is consistent with the finding that following $\mathrm{iPTX}_{6-12}$ treatment, no increases in $\mathrm{CD}^{+}$and $\mathrm{CD} 11 \mathrm{~b}^{+}$cell populations were observed in the SC despite peripheral antigen-specific responses being similar to that of iUT mice.

There are many ways by which PTX could inhibit cell migration. Leukocyte migration involves a number of complex processes and microtubules have multiple roles in these events (Friedl et al., 2008; Sanchez-Madrid et al., 1999; Watanabe et al., 2005). While it is possible that PTX was inhibiting expression of adhesion molecules necessary for the migration of $\mathrm{T}$ cells and macrophages, this explanation is unlikely given that it has previously been shown that even high concentrations of PTX $(10 \mu \mathrm{M})$ have no effect on monocyte adhesion to endothelial cells (Mamdouh et al., 2008). However, in vitro PTX has been found to directly inhibit cellular chemotaxis (Roberts et al., 1982). PTX can also hinder myeloid cell shape remodelling which is required for tissue infiltration (Lautenschlager et al., 2009). Additionally, both transcellular and paracellular leukocyte 
migration can be inhibited by PTX acting on endothelial cells (Mamdouh et al., 2008; Mamdouh et al., 2009). This research indicates that PTX can have a direct effect on the mechanics of leukocyte migration as well as processes involved in cellular extravasation. It is likely that the effects of in vivo PTX treatment on migration, observed in the current study, are caused by similar mechanisms.

In addition to altering migration, it was demonstrated that PTX could completely inhibit antigen-specific proliferation within the blood and the spleen of immunised mice. Additionally, PTX was found to limit antigen-specific T cell proliferation within the LNs that drain the EAE immunisation sites. It is therefore likely that the alterations $\mathrm{CD} 4^{+}$and GR $-1^{+}$cell populations following PTX treatment are due to inhibition of proliferation. It is interesting that some proliferation occurred within the LN but not spleen or blood of iPTX mice. An explanation for this could be that PTX might reach much higher concentrations within the blood and spleen compared to that of the dLNs. It is possible that the concentrations required to inhibit $\mathrm{T}$ cell proliferation within the dLNs were not reached until multiple injections of PTX had been given or that the once-daily-dosing leads to peaks and troughs of PTX concentrations within the LN. It has been shown that within 18 hours of i.p. injection, PTX levels within the plasma drop to less than $10 \%$ of peak plasma levels (Tsai et al., 2007). While studies have not been conducted into the levels reached within the inguinal LNs, data from other organs, such as spleen and liver, indicate similar reduction in PTX concentrations over time (Soma et al., 2009). Peak drug concentrations within the dLN would be lower than those within the blood and spleen; therefore the concentration of PTX within the dLN between daily doses is likely to be lower. This could provide a window of opportunity during which $\mathrm{T}$ cells within the dLN could proliferate. Any proliferation that was occurring during a drug concentration trough would be unaffected by PTX; while, any proliferation occurring during or near a peak in drug concentration would be inhibited. At the concentration of CFSE used to stain the 2D2 cells, multiple cycles of proliferation can be detected before CFSE fluorescence is reduced to background levels (Parish et al., 2009; Quah et al., 2007). It can be seen from both the representative plots and the number events within each generation of proliferation (Figure

4.13, a and d) that while far fewer proliferative events occurred in PTX treated mice, cells 
that did proliferate did so multiple times, supporting the concept that there may have been multiple windows of opportunity for 2D2 cells to proliferate. To verify this, the in vivo proliferation assay could be repeated, with PTX administered every other day. If the proliferation was due to drug concentration troughs, it would be expected that more proliferation would occur in dLN cells due to the extended period between drug treatments.

The complete inhibition of proliferation in antigen-specific $\mathrm{T}$ cells within the spleen but only partial reduction within the $\mathrm{dLN}$ is consistent with the results observed when spleen or dLN cells were isolated from $\mathrm{iPTX}_{\mathrm{d} 0-4}$ treated mice 5 days p.i.. In splenocytes from these mice antigen- specific proliferation and production of IFN- $\gamma$ and IL-17 did not occur following in vitro stimulation. In contrast lymphocytes from the same mice exhibited antigen-specific proliferation and production of IFN- $\gamma$ and IL-17, albeit at reduced levels, relative to iUT mice. This difference in responses between spleen and dLN is likely to be associated with the difference in anti-proliferative activity of PTX within the spleen and dLN. If so, it is conceivable that if PTX treatment had inhibited the encephalogenic responses in the dLNs to the level that occurred within the spleens, development of EAE could have been halted or further delayed.

While iPTX $_{\mathrm{d} 0-4}$ treatment resulted in inhibition of the expansion of $\mathrm{CD}^{+}$and $\mathrm{Gr}-1^{+}$ populations, the $\mathrm{F} 4 / 80^{+}$macrophage population was also significantly smaller than that from iUT mice. As both $\mathrm{Gr}-1^{+}$and $\mathrm{CD}^{+}$populations proliferate rapidly after EAE immunisation (Carlson et al., 2008; Targoni et al., 2001), it is likely that the inhibition in expansion of these populations is due to inhibition of proliferation. In contrast, macrophages do not generally proliferate (Jenkins et al., 2011). Thus, the inhibition of expansion of the $\mathrm{F} 4 / 80^{+}$population in iPTX treated mice must occur though some other mechanism. It is unlikely that PTX treatment reduced macrophage survival, as macrophages are viable in much higher in vitro concentrations than would be experienced in vivo (Crume et al., 2007). Therefore, the inhibition in expansion of macrophage populations is likely to be caused by an inhibition in the migration of these cells into the secondary lymphoid organs rather than alteration in proliferation of cell viability. 
By d13 p.i. both $\mathrm{Gr}-1^{+}$and $\mathrm{F} 4 / 80^{+}$populations in the spleen of $\mathrm{PTX}_{\mathrm{d} 0-4}$ treated mice had recovered, indicating that the effect of PTX on cell proliferation and migration respectively, only occurs during or shortly after drug treatment. Additionally peripheral encephalogenic responses were rapidly re-established, since antigen-specific proliferation and cytokine production were similar in $\mathrm{iPTX}_{\mathrm{d} 0-4}$ and iUT by d13 p.i. However, despite these similar

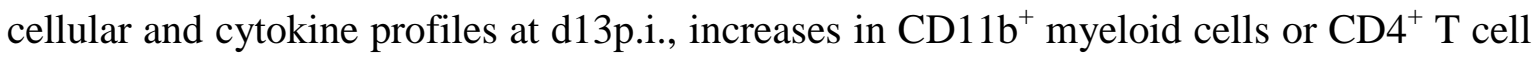
in the SC was only observed in iUT and not in $\mathrm{iPTX}_{\mathrm{d} 0-4}$ treated mice. This suggests that while peripheral encephalogenic immune responses develop rapidly following cessation of drug treatment, by d13 p.i. these are not yet sufficient to induce CNS infiltration

The initial induction of peripheral inflammatory responses occurs within the first week following immunisation and by $\mathrm{d} 6$ p.i. clonal expansion of MOG reactive T cells is already well established (Crume et al., 2009; Hofstetter et al., 2010; Targoni et al., 2001). Therefore, in contrast to the $\mathrm{iPTX}_{\mathrm{d} 0-4}$ treatment regimen, alterations in disease expression following $\mathrm{iPTX}_{\mathrm{d} 6-12}$ treatment are unlikely to be due to the inhibition of $\mathrm{T}$ cell proliferation. This conclusion is supported by the data that indicate very little difference in antigenspecific responses 13 days p.i. in spleen and dLN cells from iUT and iPTX $\mathrm{d}_{6-12}$ mice (Figure 4.7-10). Adding further support to this concept, no difference in $\mathrm{CD}^{+} \mathrm{T}$ cell number was detected between these groups.

Of the cell populations analysed within the spleen, only $\mathrm{Gr}-1^{+}$cells were significantly reduced in iPTX $_{\mathrm{d} 6-12}$ treated mice when compared to iUT mice. Antibody depletion of neutrophil/polymorphonuclear leukocytes (PML) has been shown to delay EAE onset without altering peripheral antigen-specific responses (Carlson et al., 2008; McColl et al., 1998). This is a possible factor that could contribute to the delay in disease onset in iPTX $_{\mathrm{d} 6-12}$ mice; however it is unlikely to be the sole cause of disease modification since Gr- $1^{+}$populations were not fully depleted from these mice. Additionally disease expression following antibody depletion of PMLs occurs within a few days following cessation of antibody administration and follows a similar course to that of untreated immunised mice (Carlson et al., 2008; McColl et al., 1998). In the current study disease expression after 
drug treatment was typically delayed for a number of days and often resulted in a milder disease score.

Except for the reduction in $\mathrm{Gr}_{-1}{ }^{+}$cell number in the spleens of $\mathrm{iPTX}_{\mathrm{d} 6-12}$ mice, similar cellular and cytokine responses were detected when thes mice were compared to iUT mice. Despite this, disease symptoms were only observed in iUT mice, and no infiltration of

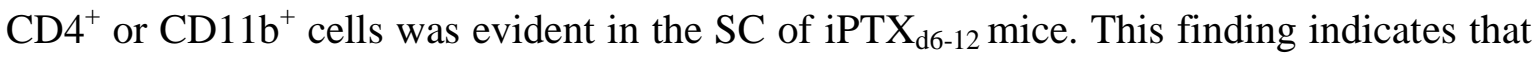
within drug treated mice the encephalogenic cells present in the periphery are not trafficking into the CNS. As migration into the CNS requires similar processes and responses to chemotatic signals to those simulated in the in vivo migration assay, the findings that PTX inhibits both T cell and macrophage migration supports the concept that PTX could inhibit migration from the periphery into the CNS.

Collectively, the findings from these experiments indicate that PTX alters immune responses to EAE immunisation when given either on d0-4 or d6-12 p.i. It is likely that PTX has a number of effects on the immune system and its therapeutic actions may differ depending on the timing of administration. While PTX administered on either d0-4 or d612 p.i. results in similar delays in disease onset, the results from the current experiments suggest that a different combination of mechanisms may lead to the alterations in disease expression. Early drug treatment temporarily inhibits expansion of $\mathrm{CD} 4^{+}, \mathrm{F} 4 / 80^{+}$and $\mathrm{Gr}-1^{+}$ cell populations. Because $\mathrm{CD}^{+}$and $\mathrm{Gr}^{+} 1^{+}$populations rapidly expand following EAE immunisation, the anti-proliferative effects of PTX would be the main contributing factor in inhibiting expansion of these populations, while an inhibition of macrophage migration is a conceivable explanation for inhibition in the expansion of $\mathrm{F} 4 / 80^{+}$populations. As was demonstrated, expansion of $\mathrm{CD}^{+}$and $\mathrm{F} / 80^{+}$populations in the spleen and $\mathrm{dLN}$ occurs within the first week following immunisation. Therefore it is not surprising the PTX $_{\mathrm{d} 6-12}$ treatment had little effect on the size of these populations. In contrast, neutrophils, which constitute the majority of the $\mathrm{Gr}-1^{+}$cells and have a rapid population turnover, are likely to be affected by iPTX $\mathrm{d} 6-12_{2}$ treatment. The reduction in the size of the $\mathrm{Gr}-1^{+}$population may be indicative of the antimitotic effect of PTXs on the proliferative processes needed to replenish this population. Therefore, while the antiproliferative actions of PTX are likely to 
contribute to alterations in disease expression when administered either on d0-4 or days 612 p.i. the relative contributions to the overall disease modifying effect may be more significant in early treatment; while PTX treatment at the later time point may be more dependent on inhibition of immune cell migration.

\subsection{Summary}

PTX administration alters encephalogenic immune responses during drug treatment. Administration of PTX immediately following EAE immunisation results in inhibition of antigen-specific responses within the spleen and attenuation within the dLN. PTX administered after induction of initial inflammatory events does not alter antigen-specific responses within either the spleen of dLN but inhibits immune cell infiltration into the CNS. Additionally five consecutive doses of PTX were demonstrated to inhibit in vivo antigen-specific proliferation and immune cell migration. 


\section{Chapter 5:}

\section{Identifying the synergistic potential of microtubule-stabilising drugs}




\subsection{Introduction}

For MSDs to be effective in treating EAE and ultimately MS, high drug concentrations and prolonged drug exposure are necessary. Additionally, it appears that while adequate drug concentrations are reached within the spleen after i.p. administration, the concentrations within the dLN may be suboptimal. Because the maximal tolerated doses of MSDs were used for the previous experiments (see chapter 3), increasing the dose administered to animals is unlikely to improve efficacy without compromising the animal's health. Therefore, in an attempt to further enhance the treatment efficacy of MSDs, two approaches based on combination treatments were explored: 1) the use of two MSDs simultaneously and 2) the use of an MSD in combination with an already approved MS treatment.

These approaches are particularly beneficial if there are synergistic therapeutic effects, in which the efficacy of two compounds used in combination is greater than would be observed for either compound alone. The potential advantages of synergy include increased therapeutic efficacy, reduced off target side effects and lower concentrations of drug required for therapeutic effects. There is already a great deal of interest in exploring the synergistic potential of current MS therapies (Conway et al., 2008; Gold, 2008). However, it is also important to consider that the use of two therapeutic compounds could potentially result in undesirable additive or synergistic adverse effects.

It has been demonstrated that combinations of different MSDs can act synergistically in inhibiting cell proliferation in vitro and within tumour bearing mice (Clark et al., 2006; Huang et al., 2006; Wilmes et al., 2007). Within in vitro tubulin polymerisation assays, synergy is often observed when two MSDs that bind to separate tubulin binding sites are combined (Hamel et al., 2006). PTX, DTX and IXB all cause microtubule stabilisation by binding to the taxoid binding site on $\beta$-tubulin. In contrast peloruside $A(P e l A)$ and laulimalide (Laul) bind to a distinct site, which does not overlap with the taxoid site (Gaitanos et al., 2004; Pryor et al., 2002). By identifying combinations of MSDs that synergize strongly, it may be possible to improve the efficacy of MSDs in the treatment of EAE and reduce any adverse effects. 
Because $\mathrm{MS}$ is a chronic disease that requires continuous treatment, it is important to consider how new MS therapies interact with the current MS drugs, such as glatiramer acetate and whether MSDs have the potential to enhance or complement the effects of these drugs. Glatiramer acetate (GA) is a well tolerated therapy already used for RRMS and helps to reduce relapse rate and slow the progression of disability. However it is only partially effective and the beneficial actions of GA can take up to 6 months to manifest (Boster et al., 2011a; Wolinsky, 2004). Pre-treating with an MSD before commencing GA treatment could potentially accelerate and/or enhance the efficacy of GA while extending the beneficial effects of MSD treatment.

\subsection{Aims and Objectives}

The current chapter investigates the potential of different MSDs to synergistically inhibit in vitro proliferation and to synergistically reduce EAE disease development in vivo. Additionally, the potential of PTX to enhance or complement the effects of GA in treating EAE was explored.

\subsubsection{Specific Aims:}

1. To identify if combinations of two MSDs act synergistically in vitro

2. To determine if combinations of MSDs can act synergistically in the treatment of EAE

3. To explore a combination treatment of PTX and GA for the treatment of EAE 


\subsection{Results}

\subsubsection{Identifying the synergic potential of MSDs to inhibit in vitro proliferation}

To quantify the anti-proliferative capacity of IXB, DTX, Laul and Pel A, splenocytes were cultured in vitro for 48 hours with Con A at various drug concentrations. Inhibition of $\mathrm{CD}^{+} \mathrm{T}$ cell and total splenocyte proliferation was measured using a CFSE dilution assay. All MSDs tested gave similar dose response profiles for both $\mathrm{CD}^{+} \mathrm{T}$ cells and total splenocyte cultures (Figure 5.1). Using the dose response curves from individual experiments, the concentrations required to inhibit $50 \%$ of proliferation $\left(\mathrm{IC}_{50}\right)$ was calculated (Table 5.1). It was found that of the four drugs tested Laul and DTX were the most potent with $\mathrm{IC}_{50} \mathrm{~S}$ of around $10 \mathrm{nM}$; while Pel A was least potent with an $\mathrm{IC}_{50}$ of around $80 \mathrm{nM}$ (Table 5.1).

Table 5.1. IC 50 values of individual MSD compounds

\begin{tabular}{lccc}
\hline Compound & \multicolumn{2}{c}{ IC50 \pm SEM (nM) } & \\
& All Cells & CD4 $^{+}$ & $\mathrm{n}$ \\
\hline Peloruside A & $86 \pm 7$ & $75 \pm 6$ & 12 \\
Laulimalide & $9 \pm 1$ & $11 \pm 1$ & 8 \\
Docetaxel & $12 \pm 2$ & $10 \pm 1$ & 4 \\
Ixabepilone & $24 \pm 3$ & $30 \pm 2$ & 8 \\
\hline
\end{tabular}

Spleen cells from C57BL/6 mice were pulsed with CFSE and cultured in the presence of Con A $(3 \mu \mathrm{g} / \mathrm{ml})$ and indicated concentrations of drug. After 48 hour incubation, cells were stained for cell surface markers and proliferation assessed by flow cytometry. The results are expressed as mean $\mathrm{IC}_{50}$ of $\mathrm{CD} 4^{+}$splenocytes or all splenocytes.

To investigate if combinations of these MSDs could act synergistically to inhibit proliferation, selected drug concentrations were combined and compared to the effects of the drugs when used individually. The inhibitory potential of combined drug concentrations was calculated using a combination index (CI). A CI value equal to one indicates additive effects of two drugs, a value greater than one indicates a antagonistic relationship and a value less than one indicates a synergistic relationship. (Berenbaum, 1985; Chou et al., 1984). 
As expected, combinations of Pel A and Laul, which bind the same site on microtubules, did not result in a synergistic inhibition of $\mathrm{CD}^{+} \mathrm{T}$ cell proliferation, (Table 5.2) although within total splenocytes synergy was detected at two different drug concentration combinations of Pel A and Laul. Notably, the greatest synergy appears at the lowest concentrations of the 2 MSDs. Combinations of Laul and DTX did not result in substantial synergy, with only one concentration acting synergistically on $\mathrm{CD}^{+} \mathrm{T}$ cells but not total splenocyte populations, while no combinations of Pel A and DTX exhibited any significant synergistic activity. In contrast, Pel A and IXB showed substantial synergy at a range of concentrations in both $\mathrm{CD}^{+} \mathrm{T}$ cells and in total splenocyte populations (Table 5.2). These studies indicate significant synergy between Pel A and IXB, but not between the other MSD combinations tested. 

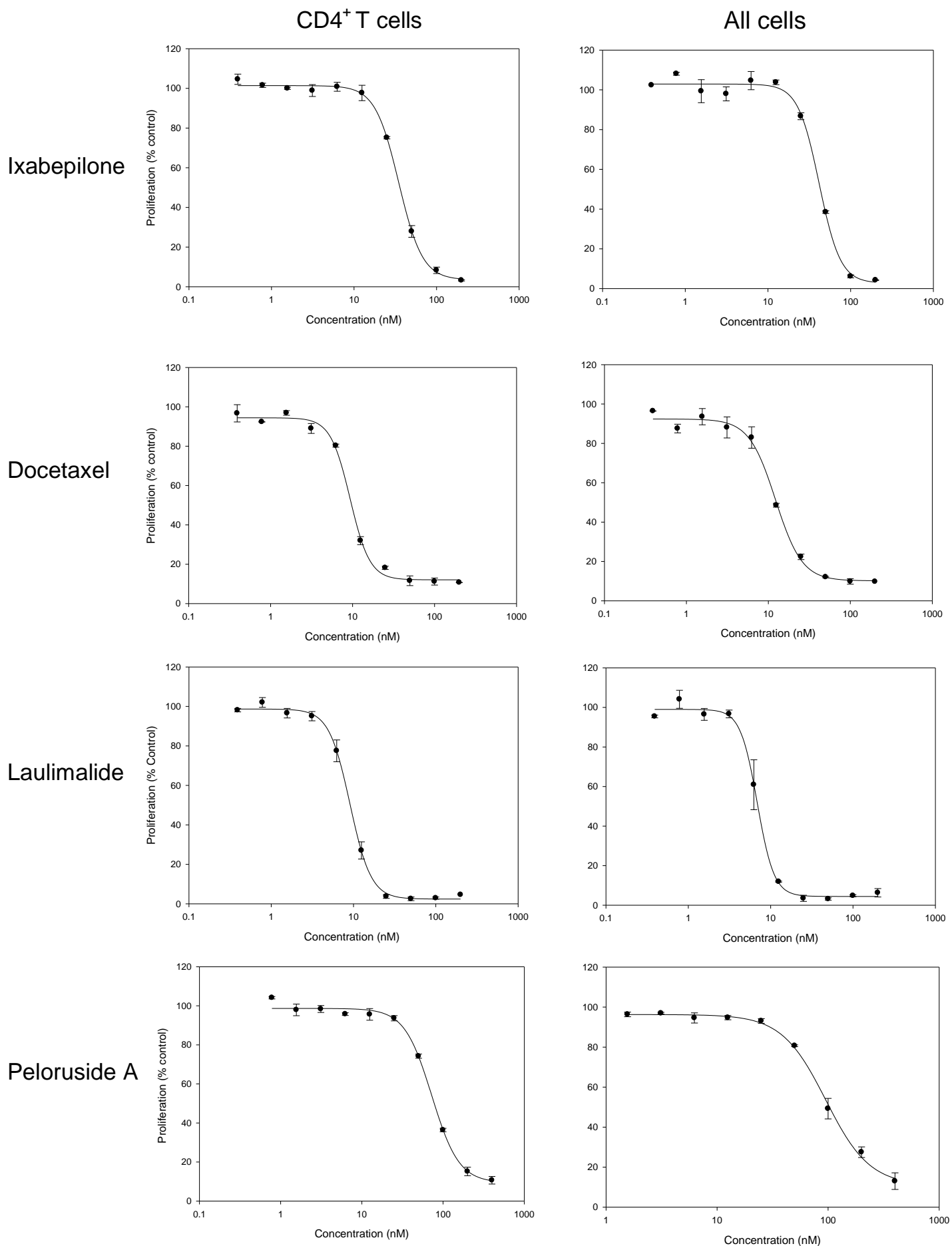

Figure 5.1. Determining the anti-proliferative activity of MSD in splenocytes

Spleen cells from C57BL/6 mice were pulsed with CFSE and cultured in the presence of Con A $(3 \mu \mathrm{g} / \mathrm{ml})$ and indicated concentrations of drug. Representative plots of $(\mathrm{a}, \mathrm{b})$ ixabepilone, $(\mathrm{c}, \mathrm{d})$ docetaxel, (e,f) laulimalide and (g,h) peloruside A. After 48 hour incubation, cells were stained for cell surface markers and proliferation assessed by flow cytometry. The results are expressed as percent proliferation of $\mathrm{CD}^{+}$splenocytes or all splenocytes relative to total $\mathrm{CD}^{+}$or total splenocytes respectively. All experiments were conducted in duplicate and at least 4 replicates were conducted per drug. 
$\underline{\text { Table 5.2. CI values for peloruside A and laulimalide drug combinations }}^{1}$

\begin{tabular}{|ll|lll|lll|}
\hline \multicolumn{2}{|c|}{ Drug combination (nM) } & \multicolumn{3}{|c|}{ All cells } & \multicolumn{3}{c|}{$\mathrm{CD}^{+}$T cells } \\
Peloruside & Laulimalide & CI \pm SEM & $\mathbf{n}$ & p-value & CI \pm SEM & n & p-value \\
\hline 50 & 6.25 & $0.97 \pm 0.05$ & 4 & n.s. & $1.08 \pm 0.14$ & 4 & n.s. \\
50 & 3.125 & $0.81 \pm 0.06$ & 4 & 0.041 & $0.96 \pm 0.12$ & 4 & n.s. \\
25 & 6.25 & $1.20 \pm 0.08$ & 4 & n.s. & $1.29 \pm 0.21$ & 4 & n.s. \\
25 & 3.125 & $0.93 \pm 0.06$ & 4 & n.s. & $0.98 \pm 0.08$ & 4 & n.s. \\
12.5 & 6.25 & $1.38 \pm 0.20$ & 4 & n.s. & $1.30 \pm 0.27$ & 4 & n.s. \\
12.5 & 3.125 & $0.77 \pm 0.09$ & 4 & n.s. & $0.79 \pm 0.09$ & 4 & n.s. \\
6.25 & 6.25 & $1.57 \pm 0.27$ & 4 & n.s. & $1.31 \pm 0.27$ & 4 & n.s. \\
6.25 & 3.125 & $0.74 \pm 0.03$ & 4 & 0.016 & & & \\
\hline
\end{tabular}

$\underline{\text { Table 5.3. CI values for peloruside A and docetaxel drug combinations }}$

\begin{tabular}{|ll|lll|lll|}
\hline \multicolumn{2}{|c|}{ Drug combination (nM) } & \multicolumn{3}{|c|}{ All cells } & \multicolumn{3}{c|}{$\mathrm{CD}^{+}$T cells } \\
Peloruside & Docetaxel & CI \pm SEM & $\mathbf{n}$ & p-value & CI \pm SEM & n & p-value \\
\hline 50 & 12.5 & $0.94 \pm 0.09$ & 4 & n.s. & $0.88 \pm 0.14$ & 4 & n.s. \\
50 & 6.25 & $1.17 \pm 0.20$ & 4 & n.s. & $1.03 \pm 0.07$ & 4 & n.s. \\
25 & 12.5 & $0.97 \pm 0.15$ & 4 & n.s. & $1.01 \pm 0.19$ & 4 & n.s. \\
25 & 6.25 & $1.14 \pm 0.14$ & 4 & n.s. & $1.21 \pm 0.11$ & 4 & n.s. \\
12.5 & 12.5 & $1.16 \pm 0.13$ & 4 & n.s. & $1.19 \pm 0.28$ & 4 & n.s. \\
12.5 & 6.25 & $1.25 \pm 0.18$ & 4 & n.s. & $1.26 \pm 0.07$ & 4 & n.s. \\
6.25 & 12.5 & $1.30 \pm 0.10$ & 4 & n.s. & $1.25 \pm 0.25$ & 4 & n.s. \\
\hline
\end{tabular}

$\underline{\text { Table 5.4. CI values for peloruside A and ixabepilone drug combinations }}$

\begin{tabular}{|ll|lll|lll|}
\hline \multicolumn{2}{|c|}{ Drug combination (nM) } & \multicolumn{3}{c|}{ All cells } & \multicolumn{3}{c|}{ CD4 $^{+}$T cells } \\
Peloruside & Ixabepilone & CI \pm SEM & $\mathbf{n}$ & p-value & CI \pm SEM & n & p-value \\
\hline 50 & 12.5 & $0.84 \pm 0.04$ & 4 & 0.028 & $0.67 \pm 0.08$ & 4 & 0.029 \\
50 & 6.25 & $0.81 \pm 0.09$ & 3 & n.s. & $0.72 \pm 0.05$ & 3 & 0.036 \\
25 & 12.5 & $0.81 \pm 0.05$ & 4 & 0.038 & $0.76 \pm 0.05$ & 4 & 0.018 \\
25 & 6.25 & $0.67 \pm 0.01$ & 3 & 0.001 & $0.74 \pm 0.06$ & 3 & 0.045 \\
12.5 & 12.5 & $0.72 \pm 0.07$ & 4 & 0.030 & $0.74 \pm 0.06$ & 4 & 0.019 \\
6.25 & 12.5 & $0.78 \pm 0.10$ & 4 & n.s. & $0.98 \pm 0.12$ & 4 & n.s. \\
\hline
\end{tabular}

Table 5.5. CI values for laulimalide and ixabepilone drug combinations

\begin{tabular}{|ll|lll|lll|}
\hline \multicolumn{2}{|c|}{ Drug combination (nM) } & \multicolumn{3}{c|}{ All cells } & \multicolumn{3}{c|}{ CD4 $^{+}$T cells } \\
Laulimalide & Ixabepilone & CI \pm SEM & $\mathbf{n}$ & p-value & CI \pm SEM & $\mathbf{n}$ & p-value \\
\hline 12.5 & 12.5 & $1.21 \pm 0.27$ & 4 & n.s. & $1.14 \pm 0.12$ & 4 & n.s. \\
12.5 & 6.25 & $1.08 \pm 0.11$ & 3 & n.s. & $0.97 \pm 0.12$ & 4 & n.s. \\
6.25 & 12.5 & $0.94 \pm 0.11$ & 4 & n.s. & $0.97 \pm 0.12$ & 4 & n.s. \\
6.25 & 6.25 & $1.12 \pm 0.15$ & 4 & n.s. & $1.03 \pm 0.15$ & 4 & n.s. \\
3.125 & 25 & $0.81 \pm 0.13$ & 4 & n.s. & $0.82 \pm 0.05$ & 4 & 0.038 \\
3.125 & 12.5 & $0.81 \pm 0.08$ & 4 & n.s. & $0.82 \pm 0.07$ & 4 & n.s. \\
1.263 & 12.5 & $1.03 \pm 0.15$ & 4 & n.s. & $0.80 \pm 0.05$ & 4 & 0.036 \\
\hline
\end{tabular}

\footnotetext{
${ }^{1} \mathrm{p}$-values determined by one sample student's $t$-test
} 


\subsubsection{Peloruside A and ixabepilone act synergistically to inhibit EAE}

As robust synergy was observed between Pel A and IXB in splenocyte cultures, the effect of combining these MSDs in the treatment of EAE was explored. Like with the in vitro experiments, it was first necessary to determine effective concentrations of these drugs in the EAE model. Administration of MSDs using a d6-12 p.i. QOD dosing schedule was chosen as this treatment regime was proven to be both well tolerated and effective. To determine effective therapeutic concentrations of each of these drugs, mice were immunised and treated on days 6-12 QOD with 10, 5, 2, or $1 \mathrm{mg} / \mathrm{kg} / \mathrm{dose}$ of IXB (Figure 5.2a) or Pel A (Figure 5.2b). These experiments revealed that all of the drugs lost $\sim 50 \%$ of their protective effect at the $5 \mathrm{mg} / \mathrm{kg}$ dose and were ineffective at $2 \mathrm{mg} / \mathrm{kg}$ or $1 \mathrm{mg} / \mathrm{kg}$ doses.

The in vitro experiments demonstrated that synergistic effects were most pronounced at concentrations in which a higher or equal ratio of Pel A to IXB was present. Because worldwide stocks of Pel A are very limited, an equal ratio combination of the two drugs was chosen to minimise the amount of Pel A used for the in vivo experiments. As individual administration of Pel A and IXB were similarly effective at $10 \mathrm{mg} / \mathrm{kg}$, it was expected that if the two drugs had an additive effect, the co-administration of $5 \mathrm{mg} / \mathrm{kg}$ of Pel $\mathrm{A}$ and $5 \mathrm{mg} / \mathrm{kg}$ of IXB would lead to a similar disease modification as observed when either drug was used separately at $10 \mathrm{mg} / \mathrm{kg}$. If, however, Pel A and IXB acted synergistically, increased disease protection would be observed compared to mice treated with $10 \mathrm{mg} / \mathrm{kg}$ of either IXB or Pel A alone. However, when tested, the combination of 5 $\mathrm{mg} / \mathrm{kg}$ Pel A and IXB proved to be extremely toxic with all mice dying between $\mathrm{d} 13-\mathrm{d} 15$ p.i. (Data not shown).

When a lower concentration of $2 \mathrm{mg} / \mathrm{kg}$ of Pel A and IXB was used, less toxicity was observed with $40 \%$ of the mice dying between (d15-19) (Data not shown). However, in the mice that survived, only 30\% developed any disease symptoms and these symptoms were considerably delayed in onset relative to all other groups (Figure 5.3). Despite the toxicity, no additional weight loss occurred in mice receiving the combined $2 \mathrm{mg} / \mathrm{kg}$ treatment, relative to that observed in untreated or individual drug treatment groups (Figure 5.4). Additionally, the overall disease burden of mice in the combined $2 \mathrm{mg} / \mathrm{kg}$ 
treatment was significantly reduced when compared to $10 \mathrm{mg} / \mathrm{kg}$ individual Pel A or IXB treatment groups, indicating that Pel A and IXB act synergistically in vivo (Figure 5.5). Synergistic interactions were not observed at the lower dose combinations of 0.5 or 1 $\mathrm{mg} / \mathrm{kg}$ IXB and Pel A suggesting that there is a narrow window in which these drugs synergize without inducing adverse effects (Figure 5.3).
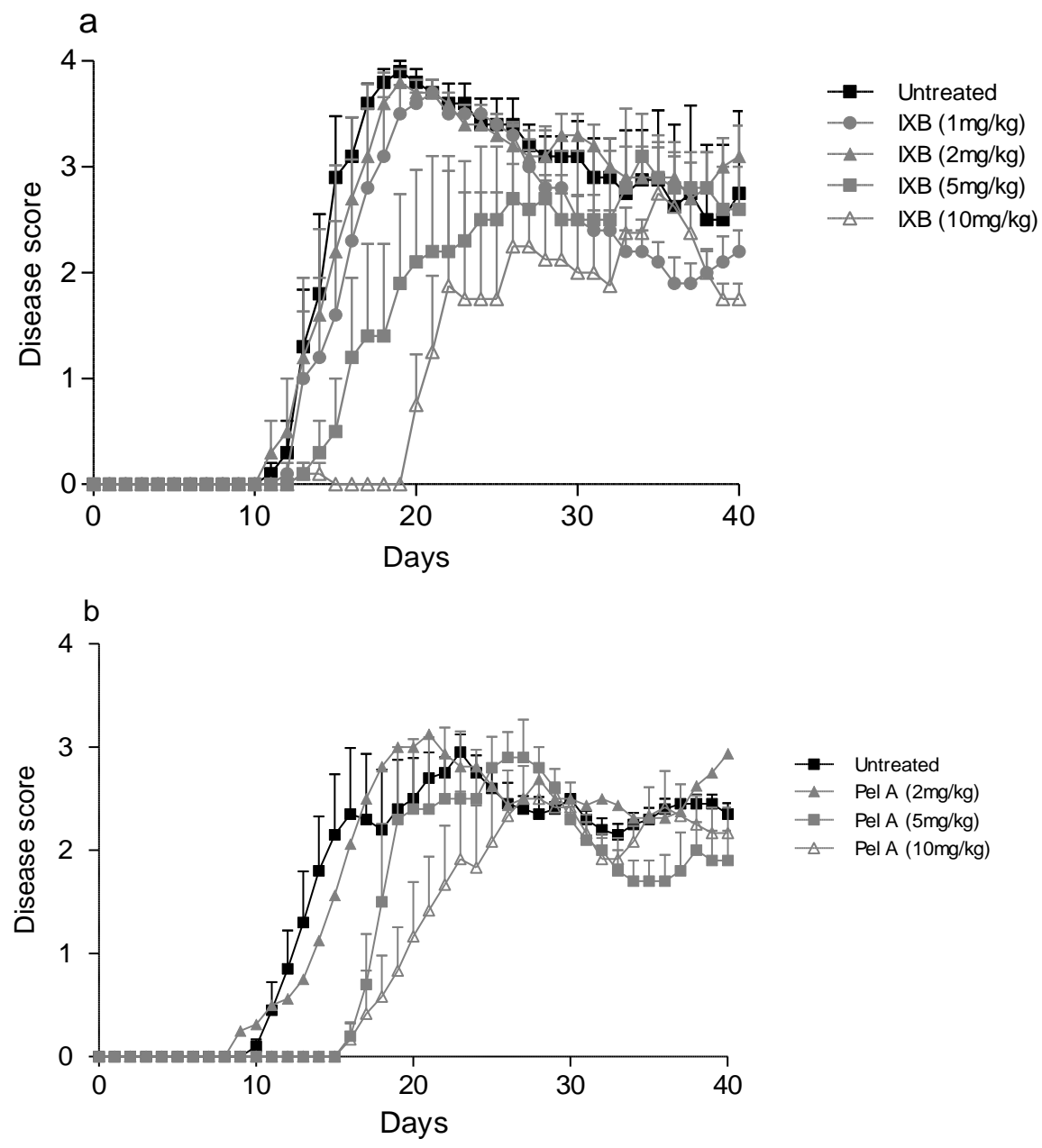

Figure 5.2. Determining the dose response effects of peloruside $A$ and ixabepilone in vivo C57BL/6 mice were immunised for EAE and left untreated or were treated with indicated treatments on days 6-12 p.i. QOD. Data represents (a) one experiment ( $n=5 /$ group) or (b) two experiments ( $\mathrm{n}=5-10$ mice/group). 

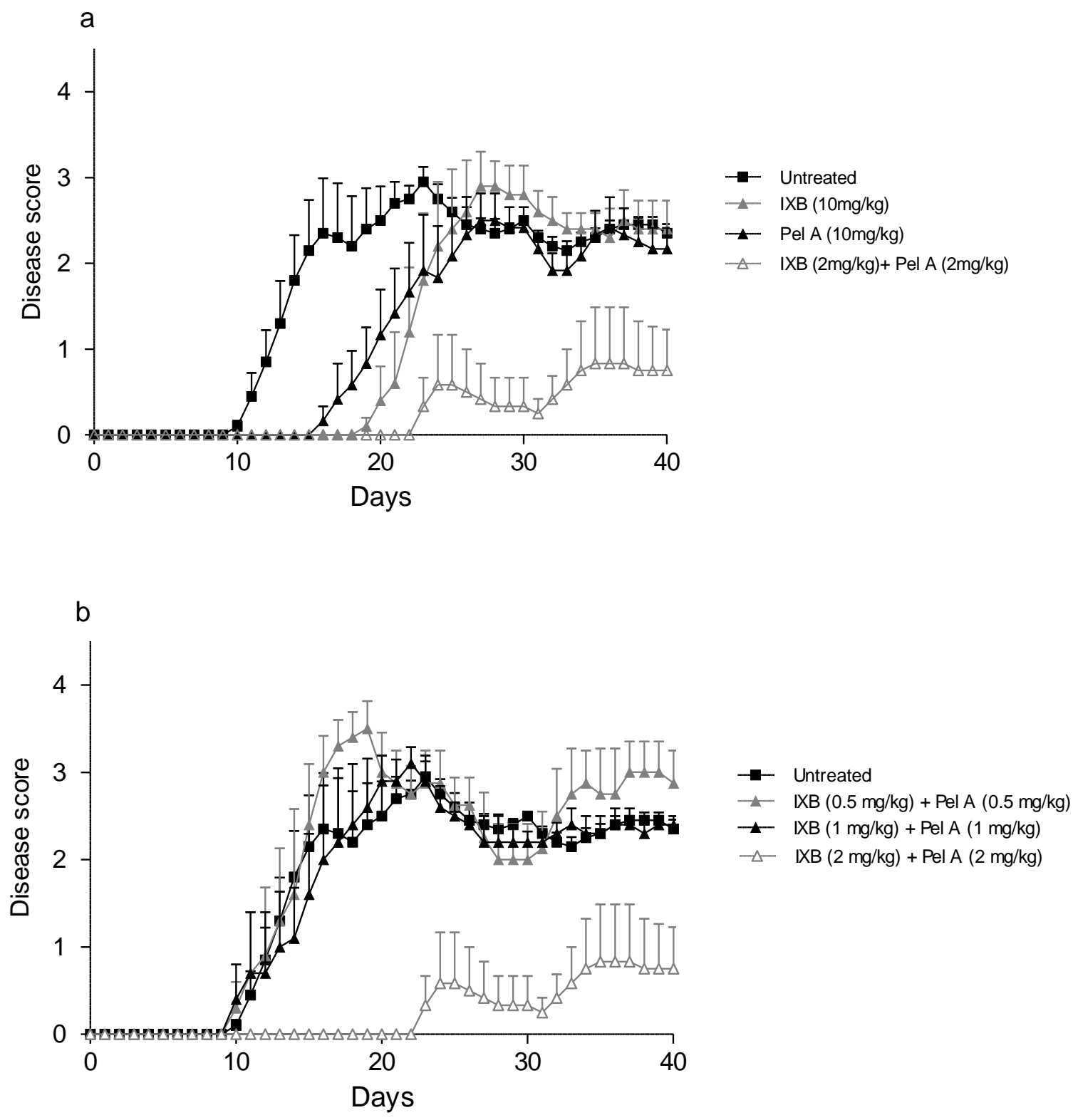

Figure 5.3 Peloruside and ixabepilone act synergistically to inhibit EAE

C57BL/6 mice were immunised for EAE and left untreated or were treated with indicated treatments on days 6-12 p.i. QOD. Figures (a,b) represent disease score of all mice from the same experiments. Data represents two experiments $(n=5-10$ mice/group). Any mice that died were excluded from the analysis. "Untreated" mice received injections of the equivalent concentrations of vehicles that would be present at the highest combined drug concentrations. 


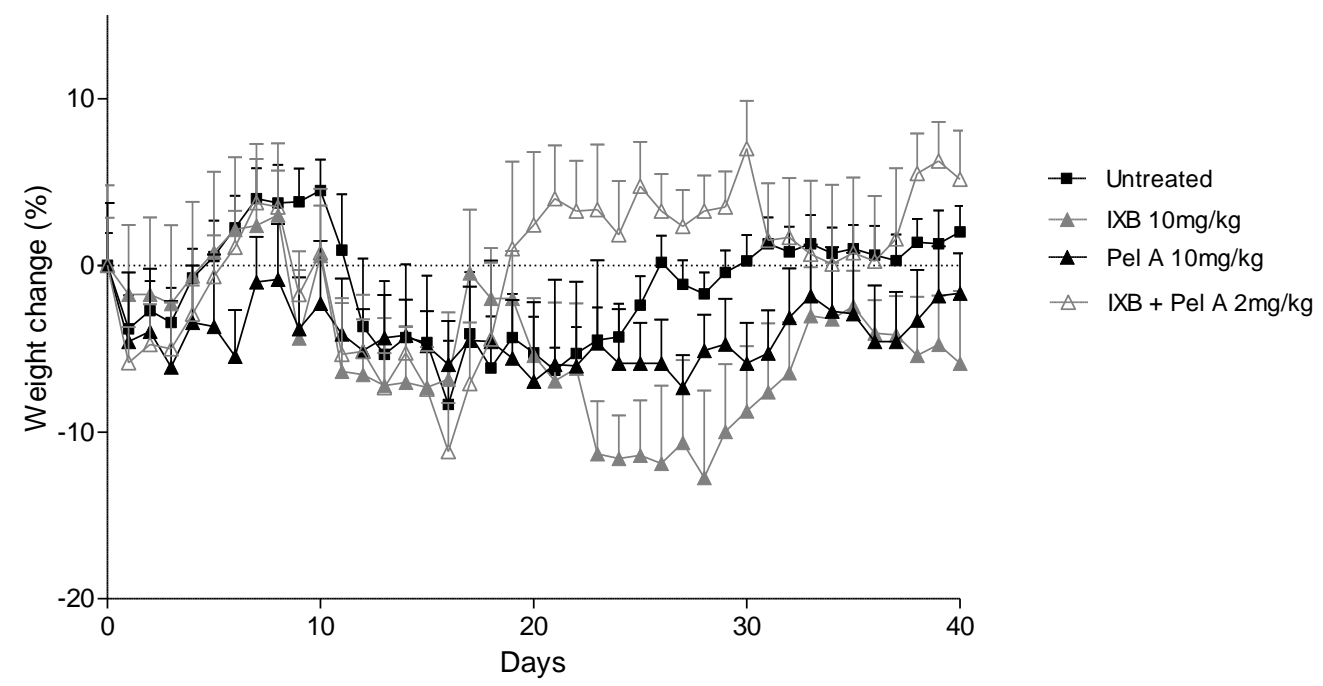

Figure 5.4. Weight change following in vivo administration of peloruside and ixabepilone C57BL/6 mice were immunised for EAE and left untreated or were treated with indicated treatments on days 6-12 p.i. QOD. Figure represents percent weight change; calculated as percent change of individual mice from weights at d0 p.i. Data represents two experiments $(n=5-10$ mice/group). Any mice that died were excluded from the analysis. "Untreated" mice received injections of the equivalent concentrations of vehicles that would be present at the highest combined drug concentrations.

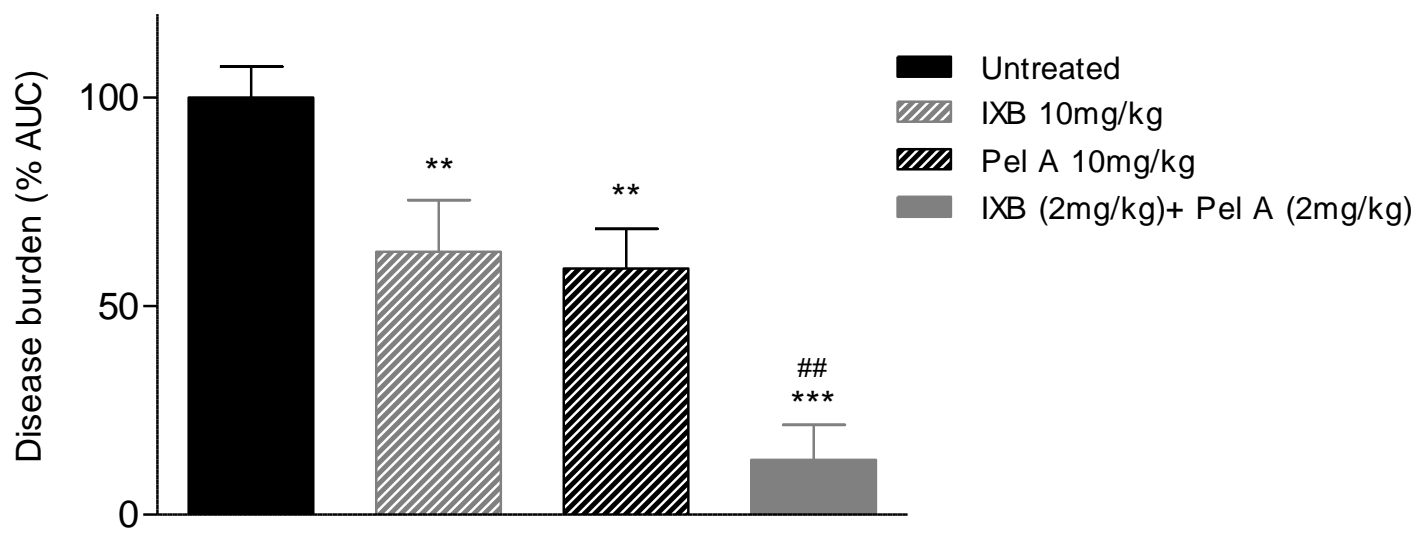

Figure 5.5 Peloruside and ixabepilone act synergistically to reduce EAE disease burden C57BL/6 mice were immunised for EAE and left untreated or were treated with indicated treatments on days 6-12 p.i. QOD. Disease burden; represented as percent area under the curve (\% AUC) relative to untreated mice. Data represents two experiments $\left(\mathrm{n}=5-10\right.$ mice/group). ${ }^{* *} \mathrm{p}<0.01$ or $* * * \mathrm{p}<0.001$ compared to untreated mice, \#\# $\mathrm{p}<0.01$ compaired to IXB $(10 \mathrm{mg} / \mathrm{kg})$ or Pel A $(10$ $\mathrm{mg} / \mathrm{kg}$ ) by one-way ANOVA with Newman-Keuls multiple comparison test. Any mice that died were excluded from the analysis. "Untreated" mice received injections of the equivalent concentrations of vehicles that would be present at the highest combined drug concentrations. 


\subsubsection{Paclitaxel pre-treatment improves the efficacy of glatiramer acetate in the treatment of established EAE.}

The current study has demonstrated that MSDs are effective at delaying the onset of EAE, but these drugs only provide a modest long-term reduction in disease severity. This transient modification of immune response induced by MSD treatment may be beneficial if used prior to an immuno-modulatory therapy. To explore this possibility, a combination treatment of PTX followed by a short term GA treatment was used. High daily doses (2 $\mathrm{mg} / \mathrm{d}$ ) of GA for 7 days before EAE immunisation can prevent disease development or, if given for 7 days following disease onset, can effectively reduce disease severity (Aharoni et al., 2010). In order to achieve a suboptimal GA treatment, a dose of $150 \mu \mathrm{g} / \mathrm{d}$ GA was used, as it had previously been shown to be ineffective in an EAE model similar to that used in the current study (Toker, 2009). In accordance with the previous study, administration of $150 \mu \mathrm{g} / \mathrm{d}$ for 7 days either before (d-6-0) EAE immunisation or following disease onset (d13-19) had no effect on disease expression (Figure 5.7a). Also, as expected, mice treated with PTX exhibited delayed disease onset and a reduction in disease burden (Figure 5.7 and 5.8). However, when PTX was administered (20 mg/kg d6-12 p.i. QOD) before GA treatment, a reduction in disease severity and overall disease burden was observed (Figure 5.7b and 5.8). This finding indicates that treating mice with PTX before GA treatment has the potential to enhance suboptimal GA responses and thus improve disease suppression. 



Figure 5.6. Paclitaxel enhances the disease modifying effects of glatiramer acetate C57BL/6 mice were immunised for EAE and left untreated (U/T) or treated with (a) glatiramer acetate (GA: $150 \mu \mathrm{g}$ ) on either d-6-d0 p.i. or d13-19 p.i. (b) or were treated with paclitaxel (PTX; $20 \mathrm{mg} / \mathrm{kg}$ ) on days 6-12 QOD, or glatiramer acetate (GA; $150 \mu \mathrm{g}$ ) on days 13-19 or a combination of paclitaxel and glatiramer acetate. Data represents mean and SEM of disease scores from all mice of (a) one experiment ( $\mathrm{n}=5$ mice/group) or (b) two experiments ( $\mathrm{n}=8-9$ mice/group). 


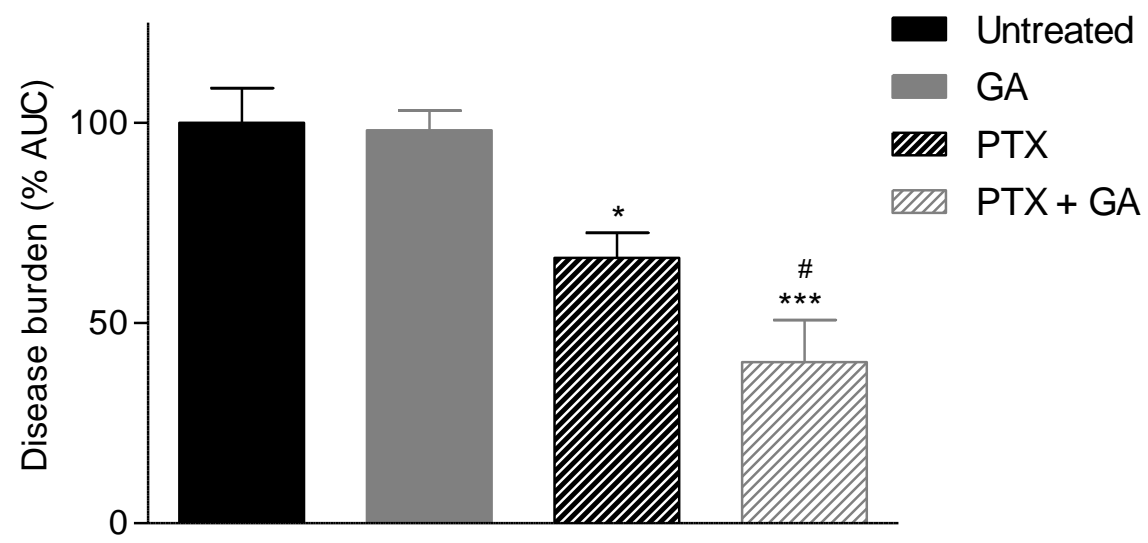

Figure 5.7 Paclitaxel and glatiramer acetate synergistically reduce disease burden

C57BL/6 mice were immunised for EAE and left untreated or were treated with paclitaxel (PTX; 20mg/kg) on days 6-12 QOD, or glatiramer acetate $(\mathrm{GA} ; 150 \mu \mathrm{g})$ on days 13-19 or a combination of paclitaxel and glatiramer acetate. Disease burden; represented as percent area under the curve (\% AUC) relative to untreated mice. Data represents two experiments ( $\mathrm{n}=8-9$ mice/group). $* \mathrm{p}<0.05$ or $* * * \mathrm{p}<0.001$ compared to untreated mice, \# p <0.05 compared to PTX by one-way ANOVA with Newman-Keuls multiple comparison test. 


\subsection{Discussion}

To further enhance the treatment efficacy of MSDs the potential for synergy between different compounds was investigated. These experiments demonstrated that combinations of Pel A and IXB exhibited substantial synergistic effects both in vitro and in vivo. Additionally, it was found that treating mice with PTX, prior to GA therapy, augmented disease suppression. Together these experiments demonstrate that although effective alone, MSD have greater potential when used as combination therapies.

Studies using purified tubulin demonstrate synergy occurs between MSDs that have different binding sites on tubulin, but not in MSDs that share the same binding site (Gapud et al., 2004; Hamel et al., 2006). However, the actions of MSDs on purified tubulin do not necessarily translate into cellular effects. This has been noted in previous cell and animal based studies, where synergistic activity can occur between MSDs that share the same binding site or conversely no synergy occurs between two MSDs that have different binding sites (Huang et al., 2006; Martello et al., 2000; Wilmes et al., 2010a). This effect was also observed in the current study as Pel A and DTX, which have different binding sites, were not synergistic. Likewise previous research in ovarian carcinoma cells found that Pel A can interact synergistically with PTX and epotholine A but not DTX, despite all three compounds sharing the same tubulin binding site (the taxoid site) (Wilmes et al., 2007; Wilmes et al., 2010a). Additionally, IXB, which also binds at the taxoid site, was found in the current study to be synergistic with Pel A in vitro. These results suggest that that synergy between MSDs is not solely due to tubulin binding interactions. A number of other factors may alter the effect of MSDs when administered in combination, such as alterations to microtubule-associated proteins, tubulin conformational effects or activity on secondary protein targets (Khrapunovich-Baine et al., 2011; Wilmes et al., 2010b). This work highlights the need for in vivo studies to identify clinically relevant synergetic interactions.

As Pel A and IXB exhibited synergy at multiple concentrations in vitro, a combination of these two MSDs was used to explore potential synergy in the treatment of EAE. The combined administration of $2 \mathrm{mg} / \mathrm{kg}$ Pel A and IXB resulted in significant inhibition of 
disease and a reduction in disease burden. This effect was far more pronounced than either of the individual $10 \mathrm{mg} / \mathrm{kg}$ Pel A or IXB treatments despite less than half of the total drug concentration being used in the combination treatment. These results demonstrate that the combination of Pel A and IXB leads to substantial synergy in vivo.

At the higher concentration of $5 \mathrm{mg} / \mathrm{kg}$ IXB/PelA severe toxicity occurred. It is likely that this toxicity was due to the synergistic effects of the drugs at this concentration. Due to the limited availability of Pel A no unimmunised mice were treated with this drug combination. Therefore it is unknown if the toxicity was a result of interactions with the EAE disease process or simply due to exceeding the maximal tolerated dose. As some toxicity was also observed in mice administered the $2 \mathrm{mg} / \mathrm{kg}$ combination treatment, a further reduction in dosing may be desirable. However, when combination of Pel A and IXB were administered at $1 \mathrm{mg} / \mathrm{kg}$ or $0.5 \mathrm{mg} / \mathrm{kg}$ no disease modification occurred. This highlights the small therapeutic window between efficacy and toxicity of IXB and Pel A when used in combination. Whether or not a concentration lower than $2 \mathrm{mg} / \mathrm{kg}$ but higher than $1 \mathrm{mg} / \mathrm{kg}$, would provide similar disease modifying effects without causing toxicity remains to be determined.

The mechanisms with which the $2 \mathrm{mg} / \mathrm{kg}$ combination treatment enhanced the inhibition of EAE were not explored. However, it is conceivable that the combined treatment had increased anti-mitotic activity in the dLN and that MOG reactive T cell proliferation was completely inhibited. Likewise the combination treatment may have inhibited cell migration for a longer period of time. A combination of DTX and Laul has been demonstrated to inhibit in vitro endothelial cell migration synergistically and at concentrations much lower than needed to inhibit proliferation ( $\mathrm{Lu}$ et al., 2006). If synergistic inhibition of immune cell migration was occurring, this could have contributed to the extended delay in disease onset that was observed in mice from the combined treatment group.

This is the first study to document synergism between Pel $\mathrm{A}$ and IXB in vivo. These findings suggest that combinations of MSDs may provide improved efficacy in inhibiting 
inflammatory disease. Further studies into the effective doses that can be used without inducing toxicity are however needed. The findings from these experiments may also be relevant to researchers interested in cancer chemotherapies. If the synergistic interactions of these two drugs cause enhanced inhibition of migration or proliferation, it likely a similar treatment would be beneficial in inhibiting tumour growth and metastases.

The other combination treatment that was explored in this chapter was the use of PTX with GA. The combined effect of GA and PTX resulted in a reduction in disease severity greater than that observed in mice treated with either GA or PTX alone. Additionally, while GA was only administered for 7 days, the reduction in disease severity was sustained well after cessation of treatment. The results from these experiments indicate that PTX pre-treatment can enhance the immuno-modulatory actions of GA.

The therapeutic effects of GA have been attributed to a number of different mechanisms. GA has been shown to promote Th2 immune response within EAE and increase production of IL-4, IL-10 and TGF- $\beta$ (Arnon et al., 2004; Kala et al., 2011). However, protection from EAE development has been demonstrated in GA-treated mice that are deficient in IL4 and/or IL-10 responses, indicating Th2 responses are not the only protective mechanism (Jee et al., 2006). Interactions of GA with monocytes can induce a type II suppressive phenotype (Toker et al., 2011; Weber et al., 2007) and GA treatment can increase Treg number and function (Jee et al., 2007). Therefore, it is likely that the immunomodulatory activity of GA is not due to one single mechanism of action but rather a combination of effects exerted on different cell types and in different tissues.

The delay in disease onset caused by PTX may provide a window of opportunity for GA to induce a regulatory response. In EAE immunised mice, increases in the Treg marker Foxp3 can be detected 4-8 days after initiation of GA treatment (Hong et al., 2005). Likewise a 23 fold increase in Treg numbers was noted within the CNS of EAE immunised mice 10 days after GA treatment (Aharoni et al., 2010). This would coincide with the delay in effect of GA observed in the current study, as it took until around d19 before disease 
symptoms in GA/PTX treated mice began to plateau and exhibit reduced disease severity compared to the PTX only treatment group.

No alteration in EAE development was observed when GA was administered for 7 days before EAE immunisation. This finding suggests that some factor associated with either the disease environment or PTX treatment enhanced the effects of GA when administered. Interestingly, it has been shown within EAE, GA induced Treg development is dependent on IFN- $\gamma$ (Hong et al., 2005). It is conceivable that GA-mediated Treg induction is therefore enhanced by the inflammatory environment that occurs during EAE. As PTX delays disease onset but does not inhibit the inflammatory milieu, administration of PTX prior to GA treatment may allow for the development of GA-induced regulatory responses before the disease becomes too severe for these regulatory process to be effective.

A number of small studies on relapsing-remitting MS patents have shown that mitoxantrone followed by GA treatment is more effective that either treatment alone (Arnold et al., 2008; Ramtahal et al., 2006; Vollmer et al., 2008). In fact, this combined treatment has recently been patented by the makers of $\mathrm{GA}^{2}$, suggesting further research will be conducted. However, as mitoxantrone has a maximal lifetime dose and causes considerable and irreversible side effects, the use of this combination is likely to be of limited therapeutic value (Neuhaus et al., 2006). Given the combined effects of PTX and GA in altering EAE disease expression, further experiments into this combination may provide an effective alternative.

\subsection{Summary:}

Collectively these results indicate that the disease modifying effects of MSDs can be enhanced either though synergistic interactions between combinations of MSDs or when used in conjunction with an immuno-modulatory compound. The findings from these experiments highlight the potential of using these drugs in combination to treat inflammatory disease.

\footnotetext{
${ }^{2}$ http://www.freepatentsonline.com/7968511.html
} 


\section{Chapter 6:}

\section{Using risperidone to treat experimental autoimmune encephalomyelitis}




\subsection{Introduction}

Risperidone is an atypical antipsychotic used to treat schizophrenia and other psychiatric disorders. The pathophysiology of schizophrenia is complex and involves alterations in brain structure as well as a dysregulation of signalling by a number of neurotransmitters, such as dopamine and serotonin (Guillin et al., 2007; van Os et al., 2009). Alterations in dopamine and serotonin signalling, within particular brain regions (e.g. the mesolimbic pathway) may have a central role in this disease (Kapur et al., 2001; Meltzer et al., 2011; van Os et al., 2009). Atypical antipsychotics, such as risperidone can block dopamine and serotonin from binding to their cognate receptors and it is though this process that antipsycotics are thought to exert their main therapeutic effects. (Kapur et al., 2001; Meltzer et al., 2011). However, psychiatric disorders such schizophrenia are often associated with alterations or dysregulation of the immune system, and it has been hypothesised that some of the therapeutic benefits of antipsychotic medication may be associated with modulation of immune parameters (Akira et al., 2003; Meyer et al., 2011; Potvin et al., 2008; Strous et al., 2006). Supporting this concept a number of studies have found risperidone and other atypical antipsychotic drugs cause alterations in serum cytokine and cellular immune responses in schizophrenic patients and animal models (Drzyzga et al., 2006; Maes et al., 2002; Muller et al., 1993). Given the potential to modulate the immune system both peripherally and within the CNS, antipsychotic drugs may prove of therapeutic benefit in inflammatory diseases. This chapter explores if risperidone modulates macrophage inflammatory responses in vitro and if in vivo administration alters disease expression of EAE.

While no studies have explored the use of atypical antipsychotics in the context of autoimmunity, it has been demonstrated that atypical antipsychotics such as risperidone, clozapine and olanzapine have anti-inflammatory effects in induced inflammation models (Basta-kaim et al., 2006; Kato et al., 2007; Sugino et al., 2009). The mechanisms of action by which these drugs alter inflammatory responses are ambiguous; however, since many immune cells respond to neurotransmitter signalling, the antagonism of neurotransmitter receptors by antipsychotics is a likely cause of these effects. Leukocytes express receptors for many neurotransmitters including dopamine, serotonin, glutamate, acetylcholine and 
catecholamines (Franco et al., 2007). Additionally it has been demonstrated that neurotransmitters including dopamine and serotonin can be synthesised and/or released by dendritic cells, macrophages and T cells (Brown et al., 2003; Cosentino et al., 2007; Flierl et al., 2007; Nakano et al., 2011; Pacheco et al., 2009; Rinner et al., 1998).

Risperidone has a strong affinity for the dopamine $\mathrm{D}_{2}$ and serotonin 5- $\mathrm{HT}_{2 \mathrm{a}}$ receptors but it also can bind to other dopamine and serotonin receptors at varying affinities (Horacek et al., 2006; Roth et al., 2003). Furthermore, risperidone has low nanomolar affinity for H1 histamine and $\alpha 1$ adrenergic receptors (Horacek et al., 2006; Roth et al., 2003). All of these receptors are $\mathrm{G}$ protein coupled receptors (GPCR), with stimulation of the $5-\mathrm{HT}_{2 \mathrm{a}}, \mathrm{H}_{1}$ or $\alpha_{1}$ receptors causing phospholipase $\mathrm{C}$ activation while stimulation of the $5 \mathrm{HT}_{1}$ or $\mathrm{D}_{2}$ receptor causes inhibition of adenylate cyclase (Jonnakuty et al., 2008; Missale et al., 1998; Piascik et al., 2001).

The role of peripherally-expressed neurotransmitters in MS is unknown, although a number of studies have noted that peripheral catecholamine (dopamine, epinephrine, norepinephrine) levels within the serum or PBMCs are altered in MS patents, particularly during active disease (Cosentino et al., 2002; Heesen et al., 2002; Rajda et al., 2002). However, the effect of increases or decreases in catecholamine levels on the immune system is likely to be dependent on the concomitant expression of particular dopamine and adrenergic receptors. Thus, further research needs to be conducted in this regard. Interestingly, one study has noted that following IFN- $\beta$ treatment sustained increases in PBMC produced catecholamines were detected, as well as increased mRNA expression for $\beta 2$ adrenergic and dopamine $D_{5}$ receptors, while $D_{2}$ mRNA expression was decreased (Zaffaroni et al., 2008). This raises the intriguing possibility that some of the therapeutic effects of IFN- $\beta$ could potentially be related to altered catecholamine signal transduction. Additionally a number of studies have documented alteration in EAE disease response following antagonism of $\mathrm{D}_{1}, \alpha_{1}, 5 \mathrm{HT}_{1}$ or $\mathrm{H}_{1}$ receptors, all of which risperidone can antagonise (Chemel et al., 2006; Dimitriadou et al., 2000; Freire-Garabal et al., 2003; Jadidi-Niaragh et al., 2010; Nakano et al., 2008; Roth et al., 2003). 
Collectively this research raises the intriguing possibility that antipsychotics such as resperidone could be beneficial for the treatment of EAE. In further support of this concept, studies using microglial cell lines have shown that risperidone or similar antipsychotic compounds can reduce the production of inflammatory cytokines such as IFN- $\gamma$, IL- $1 \beta$, IL6 and TNF- $\alpha$ (Bian et al., 2008; Kato et al., 2008; Kato et al., 2007; Zheng et al., 2008) In the context of MS microglia are involved both with pathology and repair, depending on their activation state; therefore reducing inflammatory activity of microglia may be beneficial in EAE and MS (Deng et al., 2005; Jack et al., 2005).

Macrophages and microglia share a common lineage and macrophages have been shown to express dopamine, serotonin, histamine and adrenergic receptors (Dimitrijevic et al., 2009; Reyes-García et al., 2009). It is therefore likely that similar anti-inflammatory effects of antipsychotics as those seen on microglia will also occur in macrophages. This is of particular relevance to MS, as while MS and EAE are primarily considered to be T cell mediated diseases, macrophages play a crucial role in disease induction and progression (Martiney et al., 1998; Tran et al., 1998). It has been shown that simply altering macrophage/monocyte activation within an inflammatory environment towards a less inflammatory phenotype, can inhibit EAE disease induction or reduce disease severity (Tierney et al., 2009; Toker et al., 2011; Weber et al., 2007). If antipsychotics induce a less inflammatory phenotype of both macrophages and microglia, the potential of these compounds in treating autoimmune disease would be enhanced. Therefore one of the aims of the current study is to determine if risperidone can alter macrophage activation.

Macrophages can be polarised towards different activation phenotypes depending on the environmental signals they receive. These activation states dictate how macrophages respond within a particular situation. Following exposure to an inflammatory environment or microbial products such as lipopolysaccharide (LPS), macrophages usually become classically activated and support the induction of a Th1/Th17 immune response. This activation state is characterised by increased production of inflammatory cytokines such as TNF- $\alpha$ and IL-12, as well as increased co-stimulatory molecules, such as CD40 and CD80 (Edwards et al., 2006; Qin et al., 2005). These factors are crucial for the induction of 
autoreactive T cells in EAE (Alegre et al., 2001; Grewal et al., 1998; Segal et al., 1998). However, in the presence of immune complexes or certain compounds macrophages or monocytes from an inflammatory environment can be induced to exhibit a regulatory phenotype, known as Type II activation (Anderson et al., 2002b). Type II activation is characterised by increased IL-10 and decreased IL-12 production as well as reduced expression of co-stimulatory molecules (Anderson et al., 2002b; Tierney et al., 2009). This altered phenotype has been shown to be protective in EAE (Tierney et al., 2009). Additionally Type II activation of murine monocytes and microglia has been described following administration of the MS therapeutic GA, implicating Type II activation as a factor contributing to the effectiveness of GA treatment (Pul et al., 2011; Toker et al., 2011; Weber et al., 2007).

\subsection{Aims and Objectives}

The current study investigated whether risperidone could alter macrophage activation and if this activation was consistent with a type II phenotype. To this end, macrophages were cultured under inflammatory conditions and exposed to risperidone. The effects of risperidone were compared to compounds known to alter macrophage/monocyte activation. Immune complexes (IC; consisting of $\mathrm{IgG}$ opsonised sheep red blood cells) are the prototypical inducer of type II activation; whereas GA has also been shown to induce type II activation in monocytes. Additionally, the in vivo effects of risperidone on the development of disease and autoimmune responses in EAE were investigated.

\subsubsection{Specific Aims:}

1. To determine if risperidone alters the activation state of cultured macrophages

2. To identify if risperidone is effective as a treatment for EAE

3. To explore potential changes in chronic $\mathrm{T}$ cell responses in risperidone treated mice 


\subsection{Results}

\subsubsection{Risperidone alters macrophage activation}

As EAE and MS are inflammatory conditions, it is likely that macrophages /monocytes will be biased towards classical activation. To simulate an inflammatory environment in vitro, bone marrow derived macrophages (BMM $\varnothing$ ) were incubated with IFN- $\gamma$ and then stimulated with LPS. Under inflammatory conditions both GA and IC have been shown to alter macrophage/monocyte activation towards a type II phenotype characterised by decreased IL-12 and increased IL-10 production (Kim et al., 2004; Tierney et al., 2009; Weber et al., 2007). To test if risperidone had a similar effect, macrophages were stimulated with LPS in the presence or absence of GA, IC or risperidone. As Type II activated macrophages exhibit significant differences in cytokine production by 8 hours post-stimulation and alterations in cell surface molecule expression by 24 hours, these two time points were analysed for cytokine production and co-stimulatory molecule expression, respectively (Tierney et al., 2009).

To identify if any of these compounds altered macrophage viability, an MTT assay was performed following an 8 hour incubation of the compounds with BMM $\varnothing$. The MTT assay works though cell dependent reduction of a yellow 3-(4,5-dimethylthiazol-2-yl)-2,5diphenoyltetrazolium bromide (MTT) dye to blue formazan crystals (Berridge et al., 2005). Therefore, a decrease in the formation of formazan indicates decreased metabolic activity or cellular death. Figure 6.1 illustrates that high concentrations of risperidone (100 or 200 $\mu \mathrm{g} / \mathrm{ml}$ ) resulted in a substantial decrease in MTT metabolism compared to the untreated controls, indicating that at these concentrations risperidone is toxic to macrophages. In contrast, risperidone concentrations of $50 \mu \mathrm{g} / \mathrm{ml}$ or below did not alter the metabolic activity of LPS stimulated macrophages.

Production of the inflammatory cytokine IL-12 was attenuated following treatment with 50 $\mu \mathrm{g} / \mathrm{ml}$ risperidone (Figure 6.2a). The reduction in $\mathrm{IL}-12 \mathrm{p} 40$ production was similar to that of IC-treated macrophages. While there appeared to be a trend towards decreased IL-12 production with $20 \mu \mathrm{g} / \mathrm{ml}$ risperidone, this reduction did not reach statistical significance. 
Interestingly, GA treatment did not cause any alteration in IL-12 levels. Production of the anti-inflammatory cytokine IL-10 was increased in both the 50 and $20 \mu \mathrm{g} / \mathrm{ml}$ risperidone treatment groups (Figure 6.2b); however, the increases in IL-10 were not as pronounced as the increase induced by IC treatment.

Co-stimulatory and other cell surface molecules are important in shaping the immune response elicited by stimulated macrophages. CD40, CD80 and PD-L1 are all up-regulated following classical activation by LPS (Edwards et al., 2006; Loke et al., 2003; Qin et al., 2005). In contrast, following type II activation these cell surface molecules are downregulated (Tierney et al., 2009). Similar to Type II activation, a reduction in CD40 expression is seen on risperidone $(20 \mu \mathrm{g} / \mathrm{ml})+$ LPS treated macrophages compared to LPS alone (Figure 6.3a). While there appeared to be a general trend toward reduction in CD40 by IC and GA treatment, neither of these reached significance. Likewise PD-L1 expression appeared to be reduced in risperidone, GA and IC treatments but did not reach statistical significance (Figure 6.3b). No changes in CD80 expression were observed (Figure 6.3c). Taken together these results indicate that risperidone deviates macrophage phenotypic expression away from a classical inflammatory activation state. This is evident by the reductions in the inflammatory cytokine IL-12 and co-stimulatory molecule CD40 as well as the concurrent increases in IL-10 production by risperidone treated macrophages. 


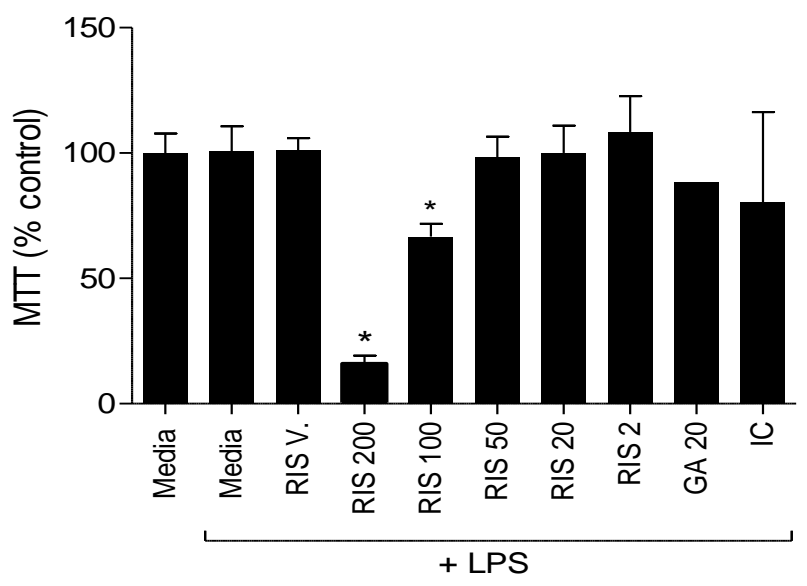

Figure 6.1 Risperidone alters macrophage viability only at high concentrations

Bone marrow derived macrophages were primed overnight with IFN- $\gamma(20 \mathrm{u} / \mathrm{ml})$ then incubated with the described treatments. Following 8 hours incubation, MTT solution was added and incubated for a further 2 hours. Data are represented as percent absorbance of untreated macrophages. Shown are means and SEM of 2 experiments conducted in duplicate. ${ }^{*} \mathrm{P}<0.05$ by one-way ANOVA with Dunnet's post hoc test; unstimulated vs. unstimulated + treatment or LPS vs. LPS + treatment. LPS (200ng/ml); RIS V (risperidone vehicle). RIS100 (risperidone, $100 \mu \mathrm{g} / \mathrm{ml}$ ); RIS 50 (risperidone, $50 \mu \mathrm{g} / \mathrm{ml}$ ); RIS 20 (risperidone, $20 \mu \mathrm{g} / \mathrm{ml}$ ); GA 20 (glatramer acitate, $20 \mu \mathrm{g} / \mathrm{ml}$ ); IC, (Immune complex, 5x106/ml).
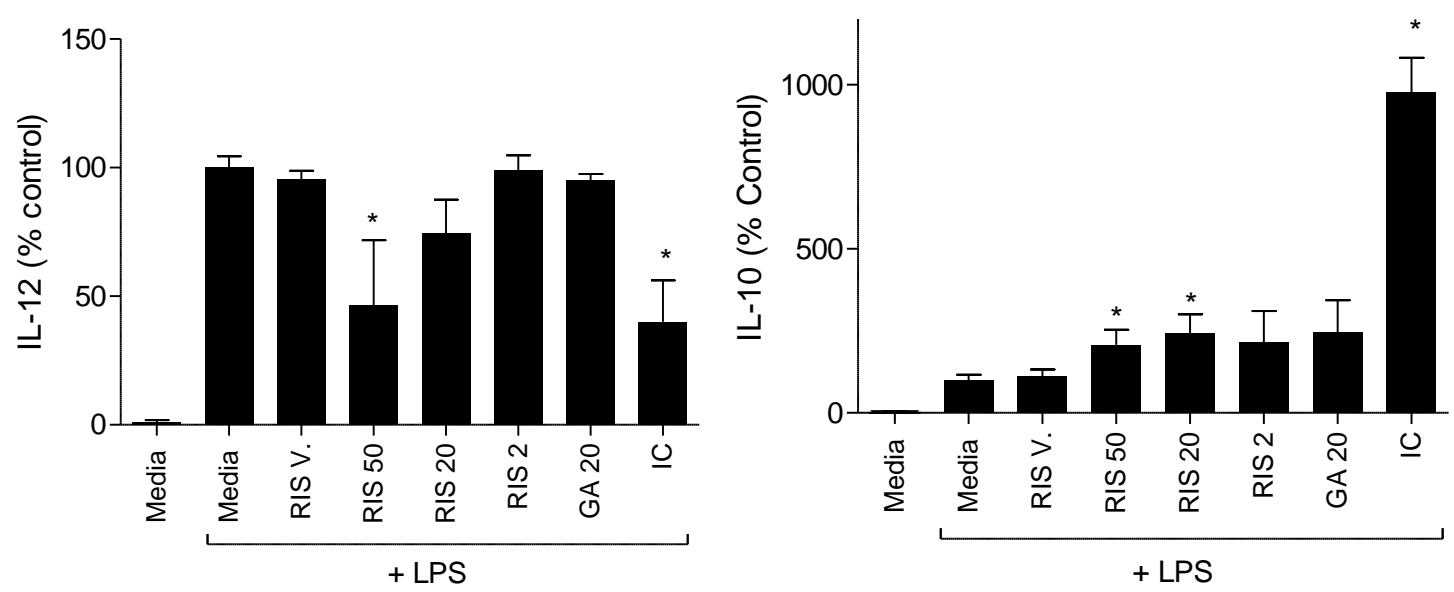

Figure 6.2 Risperidone reduces IL-12 and increases IL-10 production in LPS stimulated macrophages

Bone marrow derived macrophages were primed overnight with IFN- $\gamma(20 \mathrm{u} / \mathrm{ml})$ then incubated with the described treatments. Supernatants were removed after 8 hours and IL-10 and IL-12p40 production was assessed by ELISA. Data represented as percent change in expression relative to LPS stimulated untreated macrophages (a) IL-12p40 and (b) IL-10 production. Shown are means and SEM of 3 experiments conducted in duplicate. ${ }^{*} \mathrm{P}<0.05$ by one-way ANOVA with Dunnet's post hoc test; LPS vs. LPS + treatment. LPS (200ng/ml); RIS V (risperidone vehicle). RIS 50 (risperidone, $50 \mu \mathrm{g} / \mathrm{ml}$ ); RIS 20 (risperidone, $20 \mu \mathrm{g} / \mathrm{ml}$ ); RIS 2 (risperidone, $2 \mu \mathrm{g} / \mathrm{ml}$ ); GA 20 (glatiramer acetate, $20 \mu \mathrm{g} / \mathrm{ml}$ ); IC, (Immune complex, 5x106/ml). 

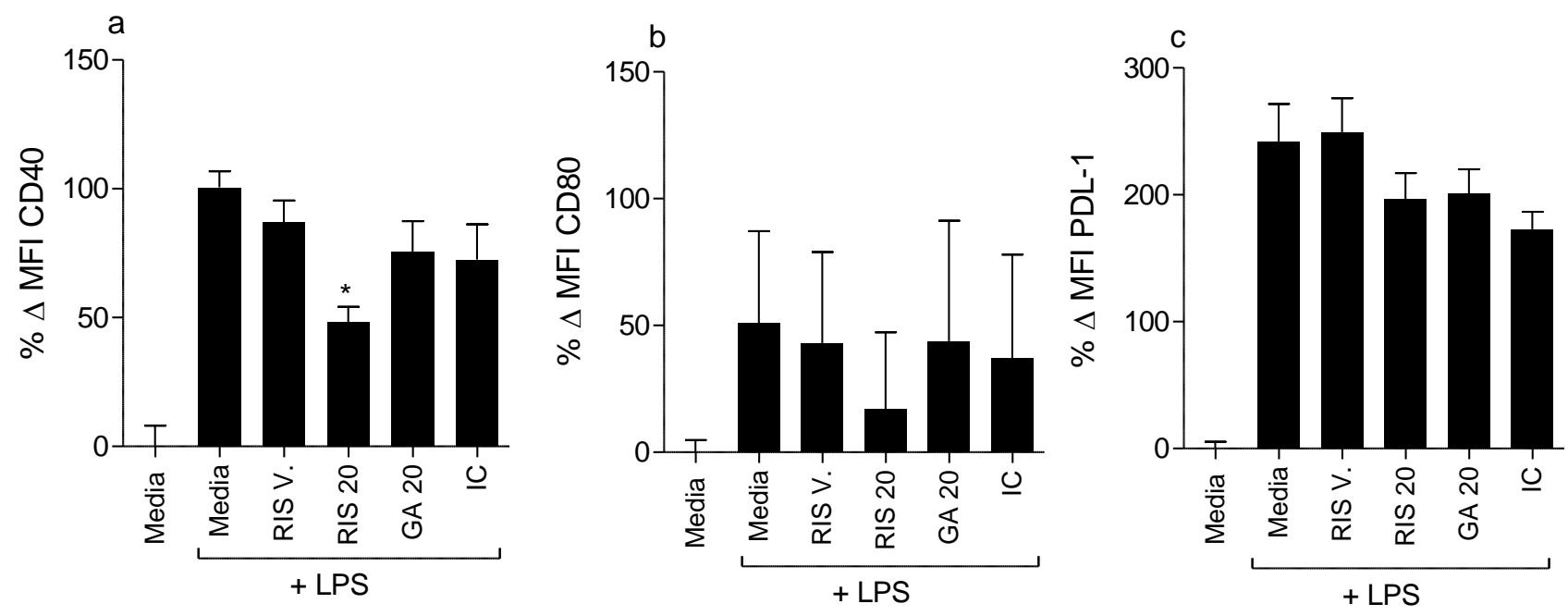

Figure 6.3 Risperidone reduces CD40 expression of LPS stimulated macrophages

Bone marrow derived macrophages were primed overnight with IFN $-\gamma(20 \mathrm{u} / \mathrm{ml})$ then incubated with the described treatments for 24 hours. Surface marker expression was assessed via flow cytometry and is represented as percentage change in mean fluorescence intensity $(\% \Delta$ MFI: MFI of specific antibody - isotype control/MFI medium alone) of (a) CD40, (b) CD80 and (c) PDL-1 expressed on $\mathrm{F} 4 / 80^{+}$macrophages. Shown are means and SEM of 2 experiments conducted in duplicate. ${ }^{*} \mathrm{P}<0.05$ by one-way ANOVA with Dunnett's post hoc test; LPS vs. RIS 200. LPS (200ng/ml); RIS V

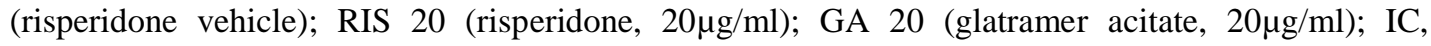
(Immune complex, 5x106/ml).

\subsubsection{Treatment of mice with risperidone reduces disease severity of EAE}

Previous studies have shown that Type II activation of macrophages or monocytes can contribute to reductions in EAE disease severity (Tierney et al., 2009; Toker et al., 2011; Weber et al., 2007). To investigate if risperidone similarly suppresses EAE disease expression, mice were treated daily with risperidone or left untreated. The doses of risperidone were chosen to be comparable to clinically relevant doses used in humans, estimated by comparable $\mathrm{D}_{2}$ receptor occupancy (Kapur et al., 2003; Karl et al., 2006; Naiker et al., 2006). To achieve a prolonged drug exposure, mice were administered risperidone via their drinking water, throughout the course of the experiments. No alteration in water consumption was observed between risperidone treated or untreated animals (data not shown). 
Risperidone treated mice exhibited reduced disease severity compared to untreated mice (Figure 6.4a). This was particularly pronounced in the $3 \mathrm{mg} / \mathrm{kg} / \mathrm{d}$ treatment group that exhibited both a reduction in disease score and overall disease burden (Figure 6.4a and c). Incidence was similar between risperidone-treated and untreated, immunised animals; however, by day 40 p.i. both $1 \mathrm{mg} / \mathrm{kg} / \mathrm{d}$ and $3 \mathrm{mg} / \mathrm{kg} / \mathrm{d}$ risperidone treatment groups had a reduced incidence of $70 \%$ compared to $90 \%$ for untreated mice (Figure 6.4b). As it appeared that there was a relationship between increased doses of risperidone and a reduction in disease score, an additional experiment using risperidone doses of 5 and 10 $\mathrm{mg} / \mathrm{kg} / \mathrm{d}$ was conducted. Interestingly, while a delay in reaching maximal disease score was observed in $5 \mathrm{mg} / \mathrm{kg} / \mathrm{d}$ treatment group, no overall reduction in incidence or maximal disease score occurred (Figure 6.5 and data not shown). Additionally no alteration in disease severity was observed in the $10 \mathrm{mg} / \mathrm{kg}$ treated mice. Thus the high concentrations of 5 or $10 \mathrm{mg} / \mathrm{kg} / \mathrm{d}$ risperidone appear to counteract the disease modifying effects observed at the lower concentration of $3 \mathrm{mg} / \mathrm{kg} / \mathrm{d}$.

When spinal cords from immunised mice were analysed, no difference in total lesion area was detected between risperidone-treated ( $3 \mathrm{mg} / \mathrm{kg} / \mathrm{d})$ and untreated sick mice (Figure 6.6). From the two experiments analysed, there was however a decrease in the number of risperidone-treated mice with lesions, when compared to the untreated group $(50 \%$ versus $80 \%$, respectively). As only one section from each spinal cord was analysed, it is possible that the reduction in lesions in risperidone treated animals was due to the limited sampling. However, in subsequent studies within our laboratory, similar reductions in lesion number has been identified in risperidone treated mice (Marie Kharhrang; personal communication), supporting the notion that risperidone treatment results in a reduction in the number of lesions. 

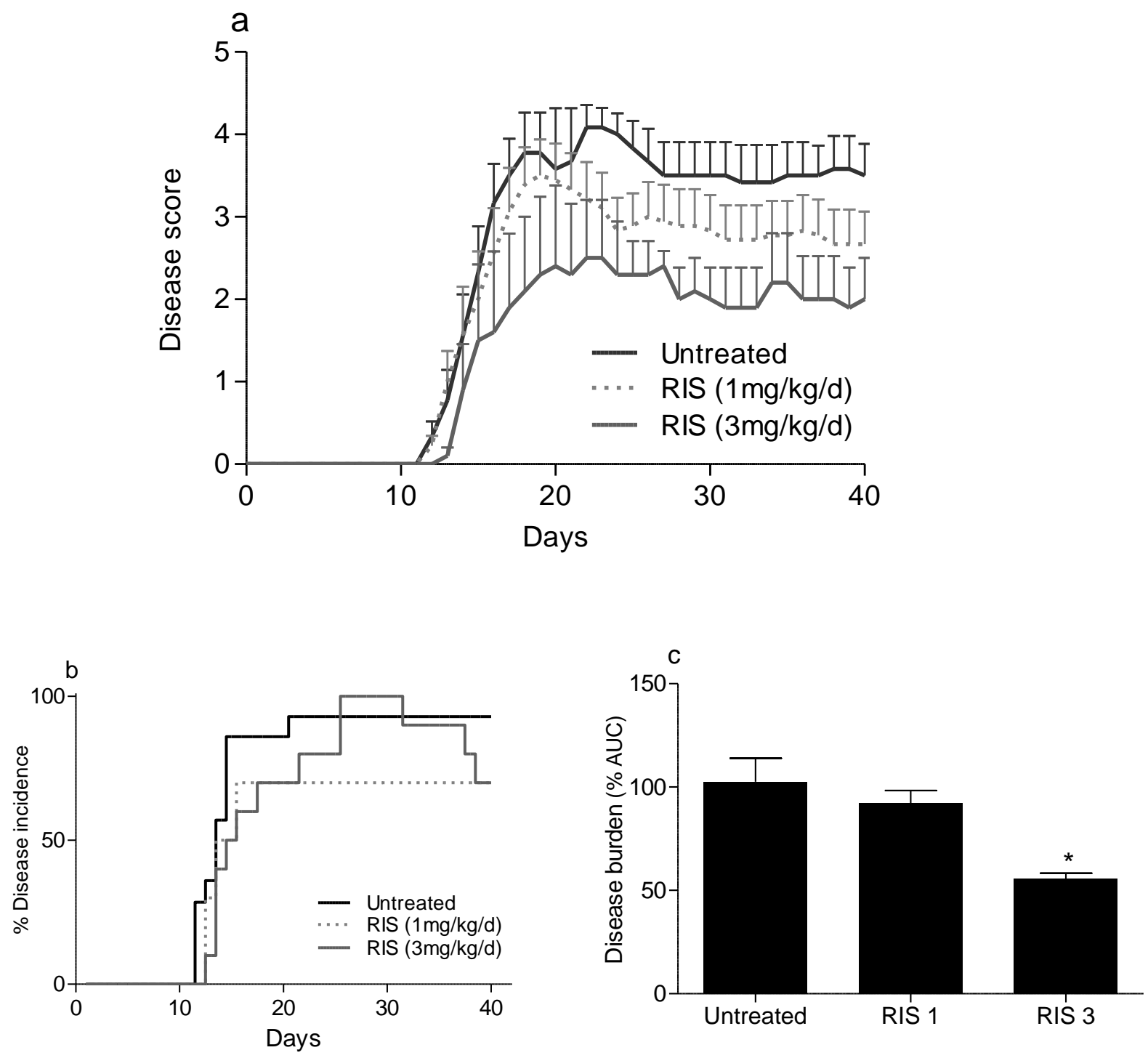

Figure 6.4 Risperidone reduces EAE disease severity

C57BL/6 mice were immunised for EAE and treated daily as indicated. (a) Disease score of sick mice. (b) Disease incidence of all mice (c) Disease burden as \% area under curve (AUC) vs untreated sick mice. Shown are means and SEM of 3 experiments ( $\mathrm{n}=9-10$ mice/group).* $\mathrm{P}<0.05$ by one-way ANOVA; with Newman-Keuls Multiple Comparison test; untreated, RIS 1 vs RIS 3. "Untreated" mice received vehicle treated water at a concentration equivalent to that which would be present at the highest drug concentration. 


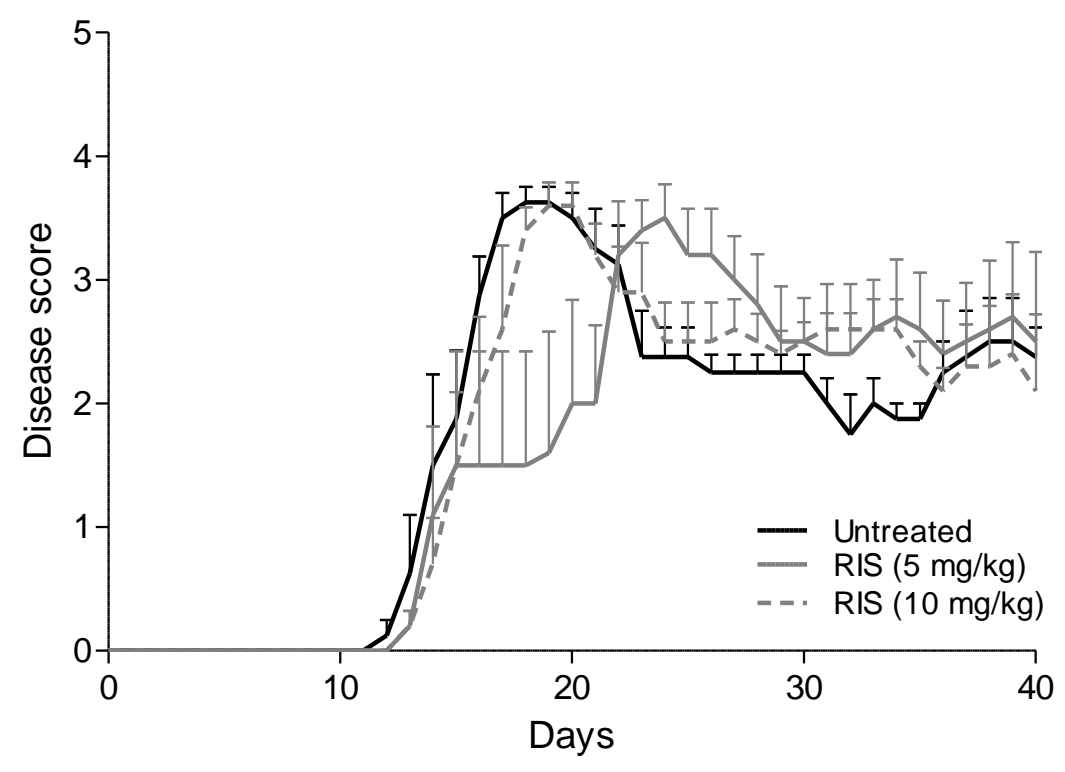

Figure 6.5 High doses of risperidone do not reduce EAE disease severity C57BL/6 mice were immunised for EAE and treated daily as indicated. Shown are means and SEM of disease scores of 1 experiment ( $n=4-5$ mice/group). "Untreated" mice received vehicle treated water at a concentration equivalent to that which would be present at the highest drug concentration.

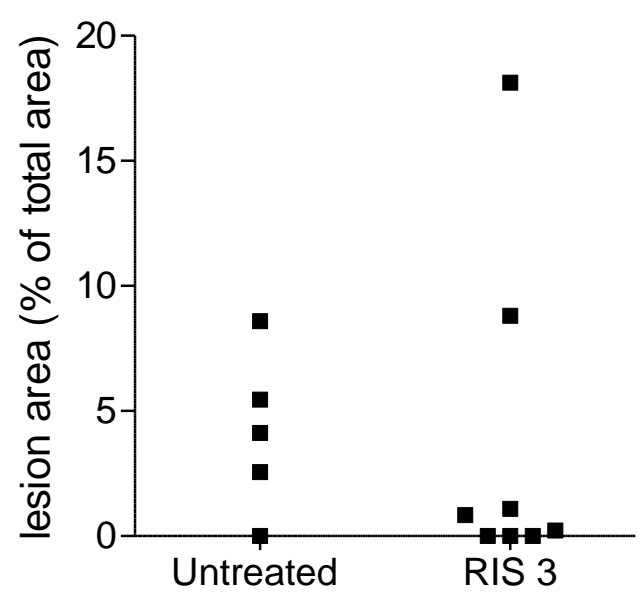

Figure 6.6 Risperidone does not significantly reduce the size of spinal cord lesions C57BL/6 mice were immunised for EAE and treated daily (RIS $3 ; 3 \mathrm{mg} / \mathrm{kg} / \mathrm{d}$ ) or left untreated. Shown are lesion areas within one section of spinal cord from individual mice. Lesion area represented as percent of total spinal cord area surveyed (see appendix 3). Only sick mice were included in the analysis ( $\mathrm{n}=5$ untreated or 8 risperidone $3 \mathrm{mg} / \mathrm{kg} / \mathrm{d}$, mice/group). No significant diffrences by a Mann-Whitney test. "Untreated" mice received vehicle treated water at a concentration equivalent to that which would be present at the highest drug concentration. 


\subsubsection{Risperidone treatment alters $T$ cell populations in the spleen}

To determine if the attenuated chronic disease responses in $3 \mathrm{mg} / \mathrm{kg} / \mathrm{d}$ risperidone-treated animals resulted in alterations in the cellular composition, splenocytes from risperidonetreated and untreated mice were isolated 40 days post immunisation and analysed for surface markers (CD4, CD25, CD8, B220, F4/80 and Gr-1). The only notable difference in cellular composition was the percentage of $\mathrm{CD}^{+} \mathrm{T}$ cells, with a higher percentage of both $\mathrm{CD} 4^{+} \mathrm{CD} 25^{+}$and $\mathrm{CD} 4^{+} \mathrm{CD} 25^{-}$occurring in risperidone-treated, immunised animals (Figure 6.7) This effect was not observed in unimmunised animals, suggesting that risperidone alone does not cause alterations in splenic cellular composition. Additionally, no difference in total number of spleen or dLN cells was observed between risperidone treated and untreated groups or between immunised or unimmunised mice (Figure 6.8).

Interestingly, despite the increased percentage of $\mathrm{T}$ cells within the spleens of immunised, risperidone-treated mice, cellular proliferation in response to Con A stimulation was significantly less, compared to splenocytes from immunised-untreated mice (Figure 6.9a). In contrast, within the dLNs immunised, risperidone-treated mice exhibited increased Con A induced proliferation compared to immunised untreated mice. Moreover, proliferation of cells from the dLN of unimmunised untreated mice was significantly higher than cells from unimmunised, risperidone-treated mice. Despite these differences in ConAstimulated proliferation, no differences in the proliferative response to MOG was observed by splenocytes or dLN cells from risperidone-treated or untreated, immunised mice (Figure 6.9b) suggesting that while polyclonal $\mathrm{T}$ cell responses may be altered by risperidone treatment, the antigen-specific $\mathrm{T}$ cell responses are not.

Analysis of cytokines within culture supernatants showed a trend toward increase IL-10 production in splenocytes from risperidone-treated mice; however, this effect was not significant (Figure 6.10a). No significant differences in production of IL-6, IL-17a, IL-4, IL-2 or TNF- $\alpha$ were observed between treated and untreated groups following either Con A or MOG stimulation (Figure 6.10b and $\mathrm{c}$ and data not shown). Interestingly, production of IFN- $\gamma$ was increased in splenocytes from immunised, risperidone-treated animals in response to MOG, but not Con A (Figure 6.10d) whereas the opposite effect 
was observed in the LN where IFN- $\gamma$ production by lymph node cells from risperidonetreated, immunised mice was increased in response to Con A but not to MOG (Figure 6.11). Taken together, these results indicate that although risperidone reduces disease, it does not inhibit antigen-specific $\mathrm{T}$ cell proliferation or cytokine production during the chronic phase of the disease. 

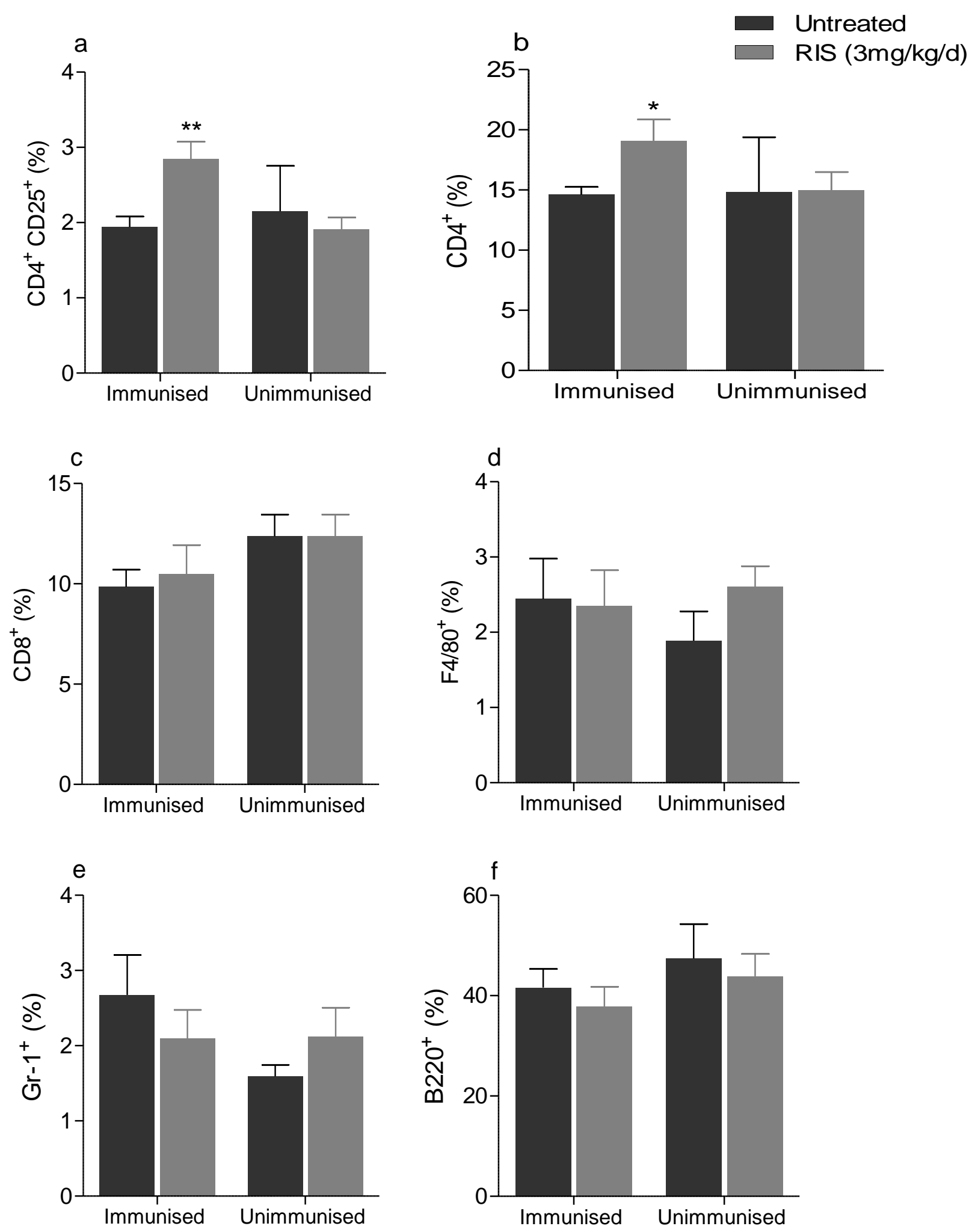

Figure 6.7 Risperidone increases the proportion of CD4 T cells within the spleen

Splenocytes were isolated 40 days p.i. and analysed by flow cytometry for percentages of total (a) $\mathrm{CD}^{+} \mathrm{CD} 25^{+}$, (b) $\mathrm{CD}^{+}$, (c) $\mathrm{CD}^{+}$, (d) $\mathrm{F} 4 / 80^{+}$, (e) $\mathrm{Gr}-1^{+}$or (f) $\mathrm{B} 220^{+}$cells. Shown are means and SEM of individual mice from 3 experiments (immunised $n=9-10$ /group) or 2 experiments (unimmunised $n=$ 4-6). * $\mathrm{P}<0.05,{ }^{* *} \mathrm{p}<0.01$ by unpaired student's t-test; immunised untreated vs immunised treated or \# $\mathrm{P}<0.05$ untreated vs. treated. "Untreated" mice received vehicle treated water at a concentration equivalent to that which would be present at the highest drug concentration. 


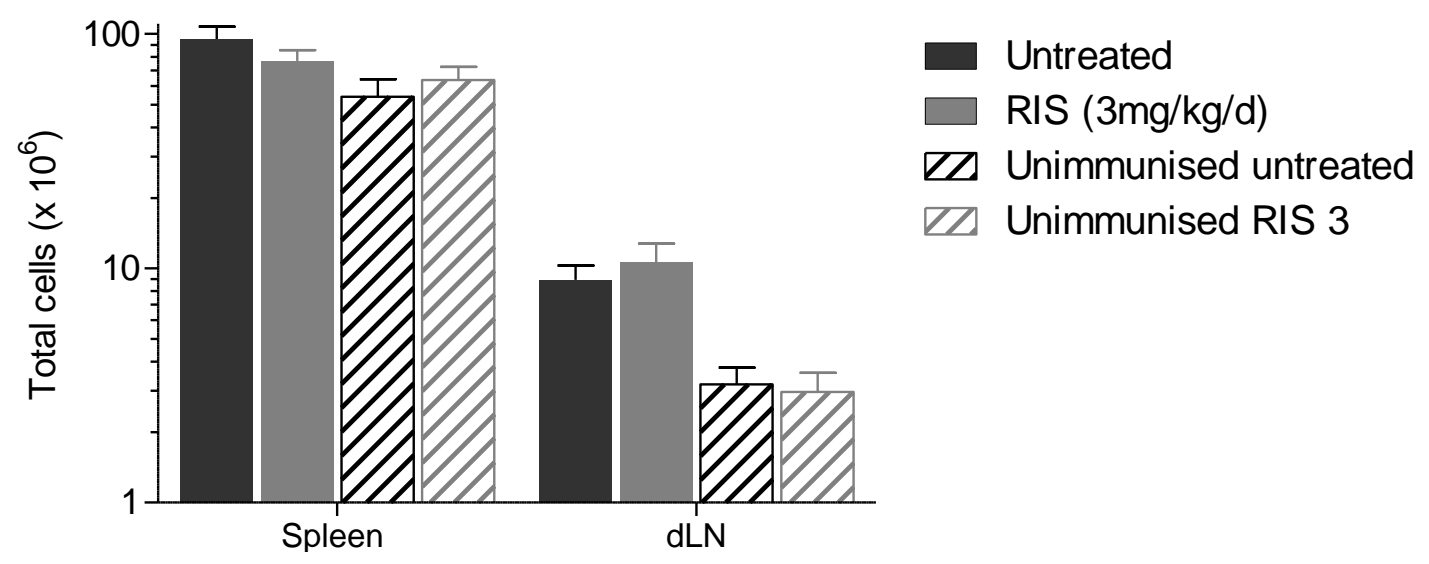

Figure 6.8 Risperidone does not alter total cell numbers in the spleen or lymph node

Spleen and lymph node cells were isolated 40 days p.i. and counted. Shown are means and SEM of individual mice from 3 experiments (immunised $n=9-10$ /group) or 2 experiments (unimmunised $n=4-6$ ). There were no significant differences between immunised untreated vs immunised treated or untreated vs. treated when assessed by an unpaired student's t-test. "Untreated" mice received vehicle treated water at a concentration equivalent to that which would be present at the highest drug concentration.
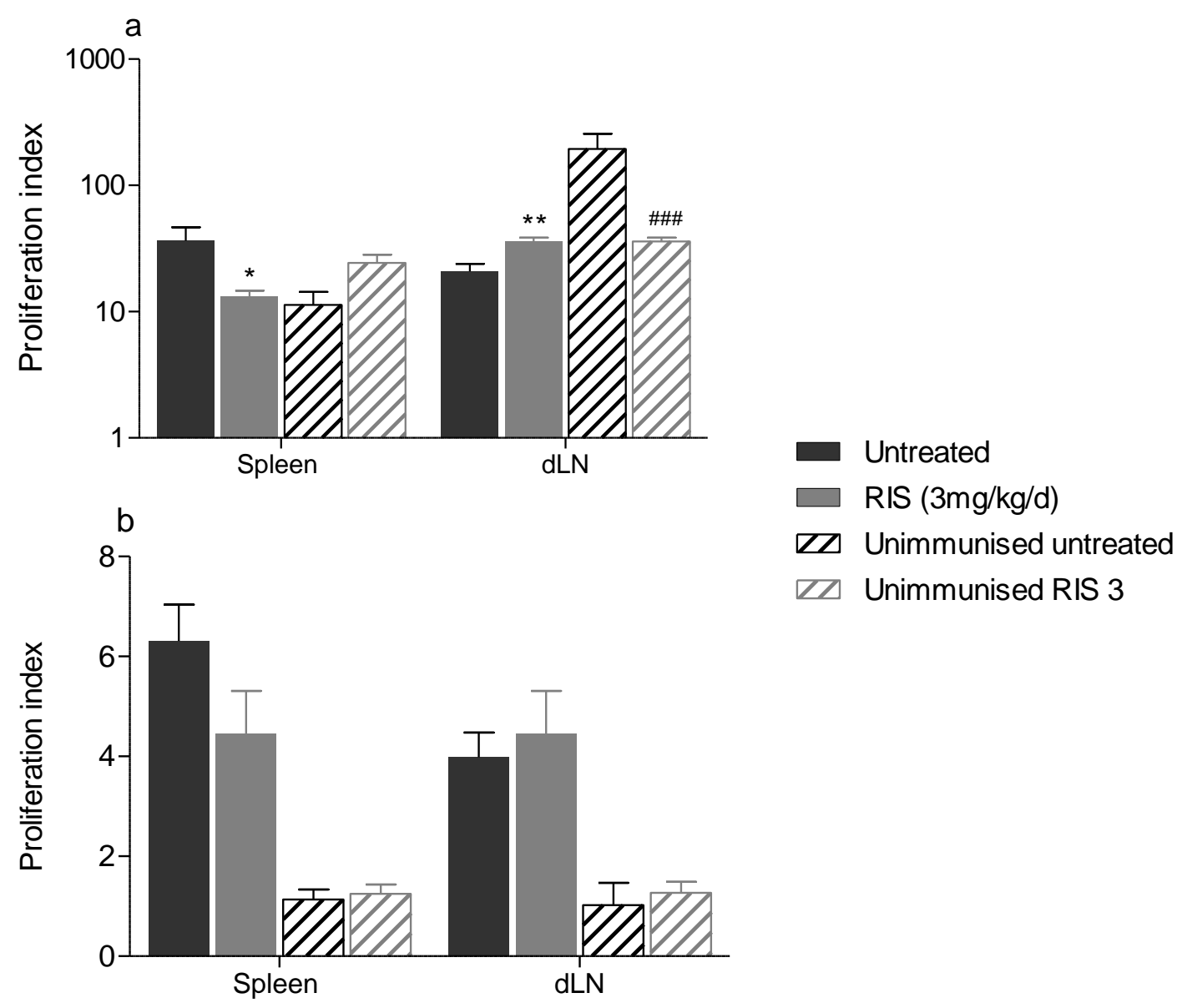

Figure 6.9 Risperidone does not alter ex vivo antigen-specific proliferation in spleen or lymph node cells Spleen or lymph node cells were cultured in medium alone or in the presence of (a) Con A ( $3 \mu \mathrm{g} / \mathrm{ml}$ ) or (b) MOG $(27 \mu \mathrm{g} / \mathrm{ml}) 40$ days p.i.. Proliferation was assessed by $3[\mathrm{H}]$ thymidine $(1 \mu \mathrm{Ci})$ incorporation and is expressed as a Proliferation index; CPM of stimulated wells/CPM of media alone. Shown are the means and SEM from individual mice from 2 experiments (immunised, $n=6-9$ ) or 1 experiment (unimmunised $n=2-3$ ). * $\mathrm{P}<0.05, * * \mathrm{P}<0.01$ by unpaired student's t-test; immunised untreated vs immunised treated or \# untreated vs. treated. "Untreated" mice received vehicle treated water at a concentration equivalent to that which would be present at the highest drug concentration. 

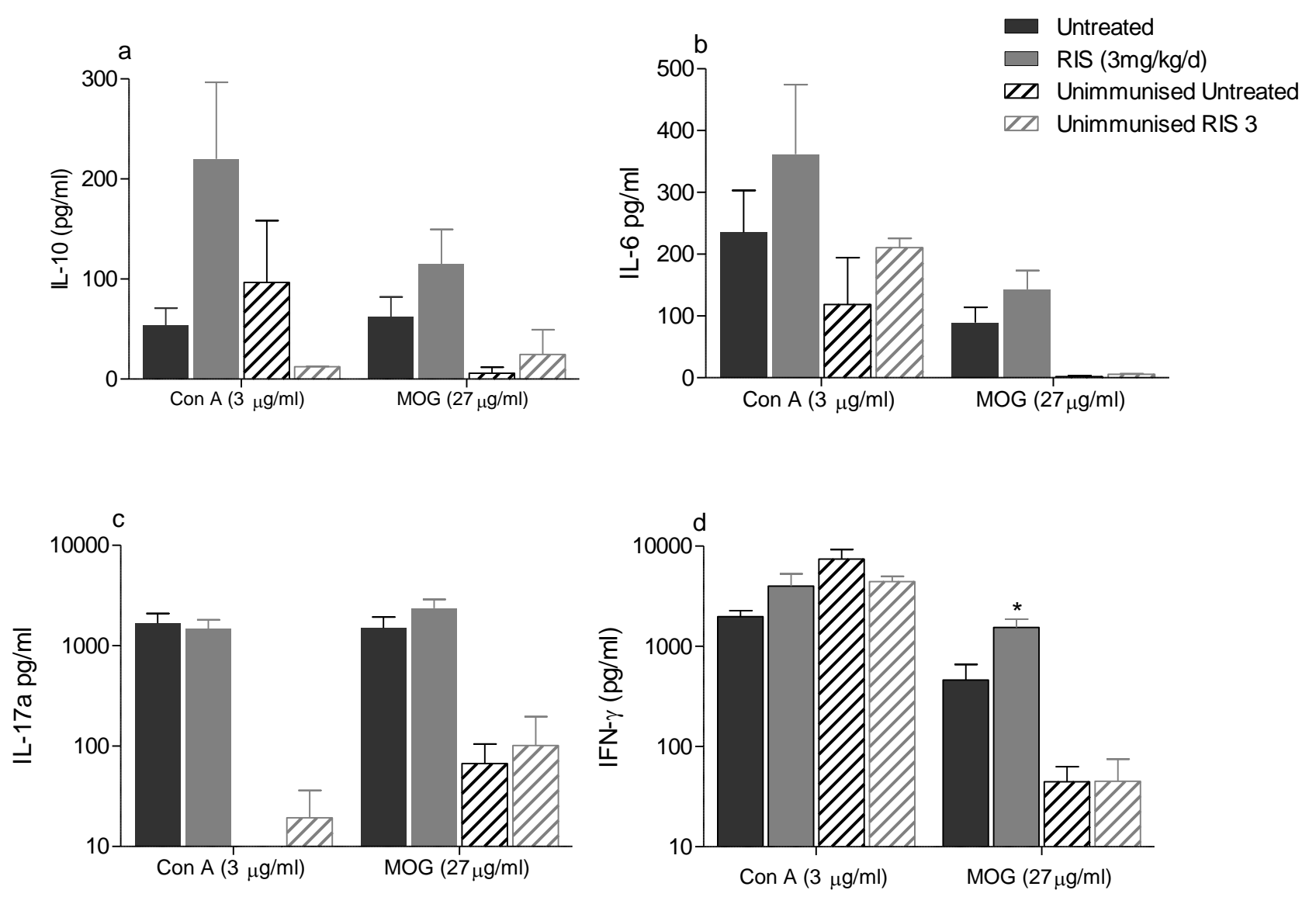

Figure 6.10 IFN- $\gamma$ production is increased following antigen-specific stimulation of splenocytes from risperidone treated mice

Forty days p.i. splenocytes were isolated and cultured in vitro and stimulated with Con A ( $3 \mu \mathrm{g} / \mathrm{ml})$ or MOG $(27 \mu \mathrm{g} / \mathrm{ml}) 48$ and 72 hours respectively. IL-10 (a) IL-6 (b) IL-17(c) and IFN- $\gamma$ (d) production was assessed by Cytometric Bead Array in culture supernatants. Shown are the means and SEM from individual mice from 2 experiments (immunised, $\mathrm{n}=6-9$ ) or 1 experiment (unimmunised $\mathrm{n}=2-3$ ). ${ }^{*} \mathrm{P}<0.05$ by unpaired student's t-test; immunised untreated vs immunised treated or untreated vs. treated. "Untreated" mice received vehicle treated water at a concentration equivalent to that which would be present at the highest drug concentration. 

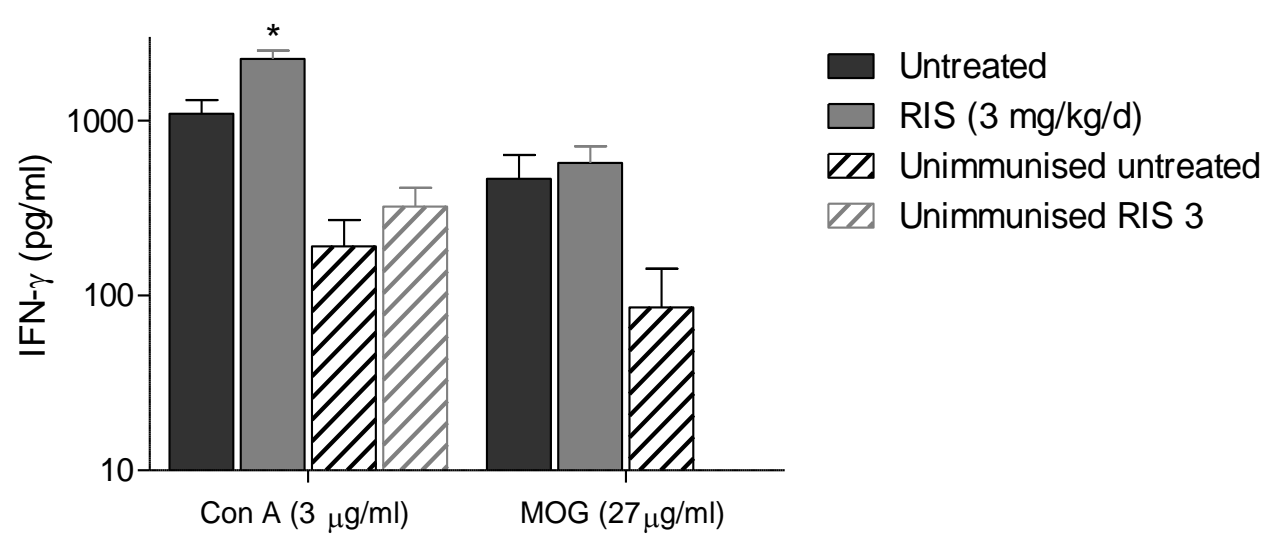

Figure 6.11 IFN- $\gamma$ production is increased following polyclonal stimulation of lymph node cells from risperidone treated mice

Forty days p.i. cells from the dLN were isolated and cultured in vitro and stimulated with Con A ( $3 \mu \mathrm{g} / \mathrm{ml})$ or MOG $(27 \mu \mathrm{g} / \mathrm{ml})$ for 48 and 72 hours respectively. IFN- $\gamma$ production was assessed by ELISA in culture supernatants. Shown are the means and SEM from individual mice from 2 experiments (immunised, $n=6-9$ ) or 1 experiment (unimmunised $\mathrm{n}=2-3$ ). $* \mathrm{P}<0.05$ by unpaired student's t-test; immunised untreated vs immunised treated. "Untreated" mice received vehicle treated water at a concentration equivalent to that which would be present at the highest drug concentration. 


\subsection{Discussion}

EAE is considered primarily a $\mathrm{T}$ cell-mediated disease; however, macrophages play a crucial role in the disease induction and progression (Martiney et al., 1998; Tran et al., 1998). Altering macrophage/monocyte activation within an inflammatory environment towards a more regulatory (type II) phenotype can inhibit disease induction or reduce disease severity (Tierney et al., 2009; Toker et al., 2011; Weber et al., 2007). As risperidone has previously been shown to induce IL-10 production and thus develop a phenotype similar to type II activation in microglia, the current study investigated whether risperidone would have similar effect on cultured macrophages.

Classically activated macrophages produce high levels of IL-12 with little IL-10 being produced (Mantovani et al., 2004; Mosser, 2003); while type II activation induces macrophages to increase IL-10 and decrease IL-12 production (Anderson et al., 2002b; Tierney et al., 2009). The current study found that supernatant from IC-treated, LPSstimulated macrophages had high levels of IL-10 and reduced levels of IL-12p40 relative to LPS-stimulated macrophages, which is consistent with previous studies (Anderson et al., 2002a; Gerber et al., 2001; Tierney et al., 2009). In contrast to previous studies; however, GA treatment had little effect on IL-10 or IL-12p40 production (Jasny et al., 2008; Pul et al., 2011). High but non-toxic concentrations of risperidone $(50 \mu \mathrm{g} / \mathrm{ml})$ resulted in a similar reduction of IL-12p40 production in LPS stimulated macrophages. Additionally IL10 production in risperidone ( 20 or $50 \mu \mathrm{g} / \mathrm{ml}$ ) treated LPS stimulated macrophages was significantly increased, relative to untreated macrophages. This indicates that like IC treatment, risperidone induces a switch from classical macrophage activation towards a more anti-inflammatory phenotype. Despite a similar reduction in IL-12 production by IC or risperidone-treated macrophages, IL-10 production was not as pronounced following the latter treatment. This suggests that risperidone is not a strong inducer of IL-10 production. Alternatively, it is possible that risperidone-treated macrophages expressed higher levels of IL-10 receptor and therefore more IL-10 was taken up from the supernatant.

The co-stimulatory molecule CD40 is expressed on macrophages and binds to CD40L (CD154) on $\mathrm{T}$ cells causing up-regulation of MHC-II and production of inflammatory 
cytokines (Suttles et al., 2009). Signalling through CD40/CD40L interactions is also assocated with the activation of antigen-specific T cell responses (Grewal et al., 1998). A reduction of CD40 expression was observed on LPS-stimulated, risperidone-treated macrophages. This reduction provides further evidence that risperidone is altering the activation state of macrophages away from an inflammatory phenotype. Within the context of EAE, CD40/CD40L interactions have been implicated as having a crucial role during disease development (Girvin et al., 2002; Grewal et al., 1996; Perona-Wright et al., 2009). Additionally in CD40 -/- mice, IL-17 production is impaired while IFN- $\gamma$ production is not, indicating CD40 may be involved with development a Th17 immune response (PeronaWright et al., 2009). Therefore, if risperidone treatment induced a down-regulation of CD40 expression on APCs in EAE immunised mice, this could result in the reduction of inflammatory cytokines, such as IL-6 and IL-12, as well as a deviation of T cells away from an encephalogenic Th17 response.

The strength of signaling though CD40 appears to alter the macrophage response, with low level signaling increasing IL-10 production while stronger signaling increases IL-12 production (Mathur et al., 2004). This suggests that the increased production of IL-10 in risperidone treated macrophages may be further enhanced following CD40/CD40L interactions. While no co-cultures of macrophages with $\mathrm{T}$ cells were conducted, it would be interesting to investigate if IL-10 production is further enhanced following CD40CD40L ligation. It has also been shown that IL-10 can inhibit up-regulation of CD40 on macrophages (Qin et al., 2006). Whether or not the reduction in CD40 expression on risperidone-treated macrophages was caused by increased IL-10 signaling in the current experiment is ambiguous.

Another important co-stimulatory molecule expressed by macrophages is CD80. CD80 provides either co-stimulatory $\mathrm{T}$ cell signaling that promotes $\mathrm{T}$ cell activation when it is bound to CD28 or an inhibitory signal when bound to CTLA-4 (Alegre et al., 2001). Upregulation of $\mathrm{CD} 80$ is associated with disease expression in EAE and blockade of CD80/CD28 interactions can prevent disease symptoms (Karandikar et al., 1998; Miller et al., 1995). A previous study found CD80 expression was modestly down-regulated on IC- 
induced type II-activated macrophages (Tierney et al., 2009). In contrast, no alterations in macrophage CD80 expression were observed following IC treatment. Additionally, neither risperidone nor GA altered CD80 expression. The low levels of CD80 expression in LPS stimulated untreated macrophages and variability in expression may have contributed to no change in CD80 being detected.

PD-L1 expression is usually associated with regulation of $\mathrm{T}$ cells though PD-1/PD-L1 interactions between T cells and APCs, respectively (Francisco et al., 2010).A lack of PDL1/PD-1 signaling has been found to exacerbate EAE (Latchman et al., 2004). Additionally, in vivo administration of GA up regulates PD-L1 on monocytes suggesting PD-L1 expression may be involved in regulation of disease processes (Weber et al., 2007). Despite the apparent regulatory role of PD-L1, expression on type II macrophages is usually down-regulated (Tierney et al., 2009). In the current experiment, no significant down-regulation was observed in IC, GA or risperidone treated macrophages; however, a trend towards reduction in PD-L1 by all three treatments is observed. What role a reduction in PD-L1 may have in type II activated macrophages is unknown, but it has been suggested that in combination with down-regulation of CD40, reduced PD-L1 could skew the T cells towards a Th2 type response (Tierney et al., 2009). Alternatively, it has been shown that in vitro blockade of PD-L1 results in increased IFN- $\gamma$ production, macrophage NO production and subsequent inhibition of $\mathrm{T}$ cell proliferation (Yamazaki et al., 2005). This may also be a mechanism whereby decreased PD-L1 could play have a regulatory role on Th1 type $\mathrm{T}$ cell responses.

Collectively these results demonstrate risperidone treatment of LPS-stimulated macrophages dampens down the inflammatory phenotype. This finding complements previous research that found similar effects of atypical antipsychotic's in microglial cultures (Kato et al., 2007; Zheng et al., 2008). Whether the effect of risperidone on macrophages constitutes a true type II activation state as observed with immune complex treatment requires more thorough investigation. However, within the limited parameters of this study, the phenotypic changes induced by risperidone are similar to those induced by IC treatment suggesting that type II activation may be occurring. 
The mechanisms by which these phenotypic changes are mediated are likely to be associated with risperidone's neurotransmitter antagonism; although it is possible that these effects are mediated though a novel mechanism of action. At the concentrations of risperidone used in the current study, both $\mathrm{D}_{1}$ and $\mathrm{D}_{2}$ type receptors would be antagonised, and it is also likely 5-HT $1,2,5,7$, histamine $\mathrm{H}_{1}$ and $\alpha$ adrenergic receptors will also be antagonised (Roth et al., 2003; Schotte et al., 1996). In contrast, it is unlikely risperidone would antagonise $\beta$ adrenergic and histamine $\mathrm{H}_{2,3,4}$ receptors, at the concentrations used.

Interestingly, a study found mouse peritoneal macrophages exposed to dopamine $(>1000$ $\mathrm{nM}$ ) had reduced IL-12 and increased IL-10 production following LPS stimulation (Haskó et al., 2002). Additionally, there was no alteration in this effect when either a $\mathrm{D}_{1}(\mathrm{SCH}-$ 23390) or $\mathrm{D}_{2}$ (raclopride) receptor antagonist was administered; however, antagonism of the $\beta_{2}$ adrenergic receptors by propranolol inhibited the effect of dopamine (Haskó et al., 2002). A similar effect of reduced IL-12 production has been observed in microglia when exposed to the $\beta_{2}$ agonist salbutamol (Prinz et al., 2001). One caveat of these studies was the possibility that dopamine was being converted to noradrenalin via dopamine-betahydroxylase. However another study in astroglial cultures using fusaric acid (a dopaminebeta-hydroxylase inhibitor) found a similar $\beta_{2}$ receptor mediated anti-inflammatory effect of dopamine, suggesting the effects observed were specifically dopamine mediated (Facchinetti et al., 2004). In the context of the current study it is conceivable that a similar mechanism could be occurring, whereby extensive receptor antagonism by risperidone results in preferential activation of $\beta_{2}$ adrenergic receptor by any catecholamine present in the culture supernatant. As macrophages or microglia can produce both dopamine and noradrenalin either of these neurotransmitters could be responsible for this effect (Brown et al., 2003; Flierl et al., 2007). However, unlike dopamine, the effects of noradrenalin on macrophage activation are more contentious, with both anti- and pro-inflammatory effects being described (Dello Russo et al., 2004; Ley et al., 2010).

Antagonism of the histamine $\mathrm{H}_{1}$ receptor is another potential mechanism by which risperidone could be eliciting its effects. On human monocytes histamine activation of the $\mathrm{H}_{2}$ (but not $\mathrm{H}_{1}$ or $\mathrm{H}_{3}$ ) receptor results in decreased TNF- $\alpha$ and IL-12 and increased IL-10 
production following LPS stimulation (Elenkov et al., 1998; Morichika et al., 2003). Additionally, histamine signaling though either the $\mathrm{H}_{2}$ or $\mathrm{H}_{4}$ receptors causes a reduction of IL-12 production in poly IC (a TLR3 agonist) -stimulated human monocyte-derived dendritic cells (Gutzmer et al., 2005). There is little work published on the $\mathrm{H}_{3}$ receptor; however, it has been noted that within human monocytes its expression is down-regulated upon differentiation (Gutzmer et al., 2005). Likewise differentiation from a monocyte to macrophage also has been noted to result in preferential expression changing from $\mathrm{H}_{2}$ to $\mathrm{H}_{1}$ receptors (Triggiani et al., 2007). While the majority of histamine is produce by mast cells, histamine is also produced by macrophages (Ghosh et al., 2002). Additionally, histidine decarboxylase the enzyme that converts histidine to histamine is up-regulated in LPSstimulated macrophages (Suzuki et al., 2009). As risperidone has strong affinity for the histamine $\mathrm{H}_{1}$ receptor but not $\mathrm{H}_{2}, \mathrm{H}_{3}$ or $\mathrm{H}_{4}$, histamine within the cultures will only be able to act via the latter receptors (Roth et al., 2003; Schotte et al., 1996). As $\mathrm{H}_{3}$ is likely to be down-regulated, histamine signaling though either $\mathrm{H}_{2}$ or $\mathrm{H}_{4}$ could contribute to a reduction in IL-12 expression and potentially increase IL-10 production.

Assuming risperidone's activity on cultured macrophages is due to receptor antagonism and subsequent activation of neurotansmiter receptors that are not antagonised by risperidone, it is possible the alterations in phenotype are associated with increases in cAMP as both $\mathrm{H}_{2}$ and $\beta_{2}$ receptors induce increases in cAMP, while the $\mathrm{H} 4$ rector, despite being considered a Gi coupled receptor, does not cause reductions in cAMP (Gutzmer et al., 2005). Thus in the current study signaling though histamine or catecholamines is likely to result in increased cAMP. cAMP has been shown in multiple studies to have a role in reducing IL-12 and increasing IL-10 production in macrophage/monocytes (Braun et al., 2001; Delgado et al., 1999; Feng et al., 2002). Future studies investigating if cAMP concentrations are increased in risperidone-treated macrophages may help to elucidate the mechanisms by which risperidone modulates macrophage activation.

The concentration of risperidone required to induce the observed changes in macrophage phenotype is higher than what is needed to induce responses following in vivo administration (Aravagiri et al., 1998). However, this difference does not preclude the 
possibility that within an in vivo context, macrophages will respond in a similar manner to lower concentrations of risperidone. This situation is possible since other factors such as CD40/CD40L interactions, risperidone metabolites or production of neurotransmitters by other cell type may influence risperidones effects on macrophages in vivo. It would be interesting in future experiments to isolate monocytes/macrophages from mice treated with risperidone to investigate if similar phenotypic alterations have occurred after in vivo treatment.

When EAE immunised mice were treated with $3 \mathrm{mg} / \mathrm{kg} / \mathrm{d}$ risperidone a reduction in disease severity and overall disease burden was observed. This concentration of risperidone is comparable to doses used clinically to treat psychophrenia (Kapur et al., 2003). Interestingly, higher doses of risperidone $(5 \mathrm{mg} / \mathrm{kg} / \mathrm{d}$ or $10 \mathrm{mg} / \mathrm{kg} / \mathrm{d})$ did not provide any protection. This finding suggests that risperidone has a dose dependent effect on the immune system. As risperidone can antagonise a number of neurotransmitter receptors, it is likely that as the concentration of risperidone increases neurotransmitter receptors which have a weaker affinity for risperidone begin to be antagonised (Aravagiri et al., 1998; Roth et al., 2003). It is therefore conceivable that high doses of risperidone may result in a different combination of receptor inhibition and thus a different alteration in immune response. An alternate explanation for the observed effects could be due to the fact that high doses of risperidone can cause extrapirimidal side effects such as 'drug-induced Parkinsonism' that is associated with postural instability and akinesia (Knable et al., 1997). As the disease severity rating scale used in the EAE model takes into account muscle tonicity, extra-pyramidal side effects may be falsely detected as EAE symptoms.

Another potential explanation for the alterations in disease expression could be related to production of prolactin, as risperidone dose dependently increases prolactin production (Bowden et al., 1992; Fitzgerald et al., 2008; Rourke et al., 2006). Exogenous prolactin has been found to promote oligodendrocyte proliferation and white matter regeneration in a lysolecithin induced demyinating mouse model (Gregg et al., 2007). Additionally, some psychotic patents have increased intracortical myelin volume following treatment with risperidone; however, it is unknown if this is due to hyperprolactinemia (Bartzokis et al., 
2007; Bartzokis et al., 2009). These observations raise the possibility that the reduction in EAE disease severity in risperidone $(3 \mathrm{mg} / \mathrm{kg} / \mathrm{d})$ treated mice is due to prolactin induced re-myelation. This however is very unlikely, as the concentrations of prolactin ( $20 \mu \mathrm{M} / \mathrm{day})$ used to induce white matter regeneration in the demyelinating mouse model, were far higher than the nanomolar levels induced by risperidone (Gregg et al., 2007; Kapur et al., 2002; Rourke et al., 2006). Likewise, if the reduction in EAE severity was associated with increased prolactin, it would be expected that the higher risperidone concentrations (5 or $10 \mathrm{mg} / \mathrm{kg} / \mathrm{d}$ ) would provide increased disease protection, as increased prolactin levels occur at higher risperidone concentrations (Rourke et al., 2006). In fact the inverse could occur whereby the lack of disease modification in the 5 and $10 \mathrm{mg} / \mathrm{kg}$ treatment groups could even be associated with increased prolactin levels since previous studies have found prolactin levels positively correlate with EAE disease severity (Esquifino et al., 2006; Riskind et al., 1991). However as these studies used either bromocriptine, ( $\mathrm{D}_{2}$ receptor agonist) or pituitary grafting to alter prolactin levels a clear causal link between prolactin and disease severity cannot be made (Esquifino et al., 2006; Riskind et al., 1991).

When the cellular composition of spleens were analysed 40 days p.i. a significant increase in the percentage of $\mathrm{CD}^{+}$cells was observed within spleens of risperidone treated mice. There was no significant difference between the ratio of $\mathrm{CD}^{+} \mathrm{CD} 25^{+}$to $\mathrm{CD} 4^{+} \mathrm{CD} 25^{-}$ indicting that this increase was not just due to expansion of $\mathrm{CD}^{+}$effector cells. Following Con A stimulation splenocyte proliferation was significantly less in the risperidone treatment group; whereas, no significant difference was observed following MOG stimulation. This result indicates that the increased percentage of $\mathrm{CD} 4^{+}$splenocytes detected within risperidone treated mice did not result in a proportional increase in $\mathrm{T}$ cell proliferative responses.

Despite the reduction in proliferation in response to Con A, no difference in IL-17 or IFN- $\gamma$ production between risperidone and untreated was observed. Additionally MOG-specific IFN- $\gamma$ production was significantly increased in splenocytes from risperidone treated mice. This finding is in partial accord with a previous study, that found that 45 days after EAE immunisation splenocytes from mice treated with a D1 receptor antagonist (SCH23390) 
produced more IFN- $\gamma$ and less IL-17 (Nakano et al., 2008). Although IFN- $\gamma$ producing Th1 cells have typically been associated with EAE disease pathology, IFN- $\gamma$ also has a protective role by limiting disease severity (Ferber et al., 1996a; Willenborg et al., 1996). Additionally Th1 cells have been shown to be less encephalitogenic than IL-17 producing Th17 cells (Murphy et al., 2010) (Berghmans et al., 2011). In the current study the increase in IFN- $\gamma$ but not IL-17 suggests a slight biasing of Th1 over Th17 type response in risperidone treated mice, and this may have a beneficial effect in limiting disease severity.

No significant differences in production of IL-6, or TNF- $\alpha$ were observed between risperidone treated and untreated mice. This contrasts with previous studies that have found reductions in plasma IL-6 and TNF- $\alpha$ levels following risperidone treatment within a necrotic pancreatitis mouse model and omega 3 deficient rats (McNamara et al., 2011; Yamaguchi et al., 2009). In the present study, cytokines were measured from supernatants of splenocyte cultures rather than plasma, and it is likely that the production of cytokines from stimulated splenocytes do not necessarly reflect the levels occurring in the plasma. This may also be true for IL-10 production, as although it appeared there was a trend towards increased IL-10 production in splenocytes from risperidone treated mice, particularly following Con A stimulation, this did not reach statistical significance. A previous study using an LPS based sepsis model showed that a single oral dose of risperidone significantly increased serum IL-10, indicating that risperidone can induce IL10 within an inflammatory environment (Sugino et al., 2009). In the current study, it is possible that IL-10 production would be more pronounced following in vitro stimulation, had risperidone been added to the cultures; or alternatively if serum cytokine production had been measured.

Collectively the results from these experiments indicate that risperidone can alter EAE disease severity, although this effect is diminished at high concentrations. Risperidone treatment did not delay disease onset or inhibit antigen-specific $\mathrm{T}$ cell responses, indicating that the reduction in disease severity is not due to inhibited induction of a disease response. It is possible, therefore, that the main disease modifying effects occur in situ within the CNS rather than in the periphery. Assuming risperidone's effects on in vitro microglial and 
macrophage cultures occurs in vivo it is likely the altered phenotype of microglial cells and macrophages would lead to a reduction in chronic CNS inflammation. Additionally the observed increase in IFN- $\gamma$ production in splenocyte may indicate stronger Th1 compared to Th17 $\mathrm{T}$ cell response, which could also assist in limiting disease severity. With future studies a more thorough investigation into potential immunological changes within the CNS may be helpful in determining potential mechanisms though which risperidone alters disease. As well as investigating if risperidone induces changes in macrophage and microglia, cytokine and surface marker expression needs to be investigated in vivo to identify if changes occur in a similar manner to those seen within in vitro cultures.

\subsection{Summary}

This is the first study to explore the effects of risperidone in EAE. The findings from this study raise the possibility that risperidone could provide therapeutic benefit in this inflammatory disease. As risperidone has been clinically used for treatment of schizophrenia for a number of years, dosing, safety and side effect profiles are well understood. It can be administered to patients orally or in slow release depots and has relatively mild side effects. All these factors suggest further investigation into the therapeutic potential for the treatment of MS is warranted. 


\section{Chapter 7:}

\section{Using MIS416 to treat experimental autoimmune encephalomyelitis}




\subsection{Introduction}

MIS416 is a novel microparticle derived from Propionibacterium acnes that is comprised of a muramyl dipeptide cell wall skeleton and bacterial DNA (Girvan et al., 2010). While originally developed as a vaccine adjuvant, interest has grown around the potential use of MIS416 as immunomodulatory agent for treatment of inflammatory disorders. MIS416 was approved for compassionate use ${ }^{3}$ and administered (i.v. once a week) to 8 patients suffering from progressive forms of MS. Anecdotal reports from these patients indicate that MIS416 is well tolerated and self-reported improvements in disease symptoms have been noted (personal communication; Gill Webster, Innate Immunotherapeutics). Additionally, given the positive results from the compassionate use, a phase $2 \mathrm{~A}$ trial is currently underway to evaluate the safety, tolerability and dosing of MIS416 within progressive MS patients. However, while the immune targets of this microparticle are known (ie. TLR9 and NOD2), the mechanism by which activating these innate receptors leads to an improvement in MS patients is not clear. The current chapter explores the potential of MIS416 to prevent or reduce disease in the EAE model of MS and investigates what mechanisms could mediate disease modification.

The muramyl dipeptide and bacterial DNA present in MIS416 principally activates the pattern recognition receptors (PRR) NOD2 and TLR9 respectively (Girvan et al., 2010). Signalling though these PRRs can result in activation of transcription factors including NF$\kappa \mathrm{B}$ and MAP kinases resulting in the production of cytokines including type I (e.g. IFN- $\alpha$ and IFN- $\beta$ ) and II interferons (i.e. IFN- $\gamma$ ), IL-6 and TNF- $\alpha$ (Akira et al., 2006; Girvan et al., 2010; Inohara et al., 2000; Magalhaes et al., 2011). Typically, acute activation of PRR causes a strong inflammatory response that is associated with the clearance of bacterial and viral pathogens (Meylan et al., 2006; Siamon, 2002). However, under certain circumstances such as robust or chronic activation PRR responses can act in an immunomodulatory manner (Marta et al., 2009; Vaknin et al., 2008).

\footnotetext{
${ }^{3}$ Compassionate use refers to the permitted use of an unapproved experimental medicine when prescribed by a medical practitioner under sections 25 and 29 of the NZ medicines Act 1989.

(for more details: http://www.medsafe.govt.nz/profs/RIss/unapp.asp)
} 
Activation of PRR has been both implicated in the development and pathogenesis, as well as, the regulation of EAE (Carty et al., 2011; Fernandez et al., 2010; Miranda-Hernandez et al., 2011; Shaw et al., 2011; Waldner, 2009). Signalling through PRRs such as the TLRs is necessary for the active induction of EAE, but what role TLR receptors have is modifying disease responses is less clear (Marta et al., 2008). Depending on the EAE induction protocols TLR9 deficiency can exacerbate or reduce disease severity, and a recent study has found NOD2 deficient mice are resistant to EAE (Marta et al., 2008; Prinz et al., 2006; Shaw et al., 2011). However, multiple studies have demonstrated that administration of TLR ligands (such as TLR7 or TLR3) have a protective effect in EAE (Hayashi et al., 2009; O'Brien et al., 2010; Touil et al., 2006). Collectively this research suggests that PRR signalling, when used in the right context, could provide a potential method for modifying disease response.

MIS416 microparticles have a unique structure that incorprates TLR9 and NOD2 agonists while being devoid of other bacterial factors (Girvan et al., 2010). MIS416 was specifically designed to induce innate immune responses while limiting local and systemic toxicity (Girvan et al., 2010). Therefore, activation of PRR by MIS416 could provide a safe and effective way to modulate inflammatory immune responses. 


\subsection{Aims and Objectives}

The current chapter explores the potential of MIS416 as a treatment for EAE and identifies effective dosing regimes with which to administer this compound. Cytokine levels within the blood were measured to determine if MIS416 causes systemic alterations in inflammatory mediators. Preliminary studies were also conducted to identify if changes to the cytokine environment contributed to the MIS416 mechanism of action.

\subsubsection{Specific Aims:}

1. To identify if MIS416 is effective at modifying EAE

2. To determine if MIS416 modifies cytokine production and how any modification contributes to its mechanism of action 


\subsection{Results}

\subsubsection{Administration of MIS416 on the day of immunisation reduces EAE disease severity and alters cytokine production}

To determine if MIS416 has any effect on the development of EAE, $250 \mu \mathrm{g}$ of MIS416 was administered i.v. to mice either 3 days prior to or immediately before immunisation. This dose and administration route was chosen for the initial experiments as $250 \mu \mathrm{g}$ i.v. had been found to be well tolerated in unimmunised mice (personal communication; Gill Webster, Innate Immunotherapeutics). Administration of MIS416 on the day of immunisation (d0 p.i.) resulted in a delay in disease onset and a reduction in disease severity (Figure 7.1a). Treatment on d0 p.i. with MIS416 also inhibited the weight loss observed in the untreated mice (Figure 7.1b). In contrast to d0 MIS416 treatment, mice injected with MIS416 3 days prior to immunisation (d-3 p.i.) exhibited no difference in disease expression or weight change compared to untreated mice (Figure 7.1 a and b). These results suggest that a single dose of MIS416 administered on the day of immunisation is effective at reducing EAE.

To investigate if MIS416 treatment altered the levels of serum cytokines, blood samples were taken from the mice at multiple time points throughout the duration of the experiment and analysed for IL-1 $\beta$, IL-2, IL-4, IL-5, IL-6, GM-CSF, TNF $\alpha$, IL-10, IL-17, IFN- $\gamma$ and TNF- $\alpha$. Of the 10 cytokines measured, only those showing differences (IFN- $\gamma$ and IL-6) have been included in the analysis (Figure 7.2). It can clearly be seen that MIS416 induces production of IFN- $\gamma$ when administered either on d-3 or on d0 p.i. (Figure 7.2a). However the kinetics of expression is different between the treatment groups with mice that were administered MIS416 on d0 p.i. having delayed peak expression of IFN- $\gamma$ compared to MIS416 d-3 p.i. treated mice. A similar effect was observed for IL-6 production with peak concentrations occurring at day 0 for d-3 p.i. MIS416 treated mice compared to day 8 for d0 p.i. treated mice (Figure 7.2b). Overall the cytokine analysis indicates that MIS416 treatment induces a select but systemic cytokine response that peaks within 4-8 days post injection of MIS416. 

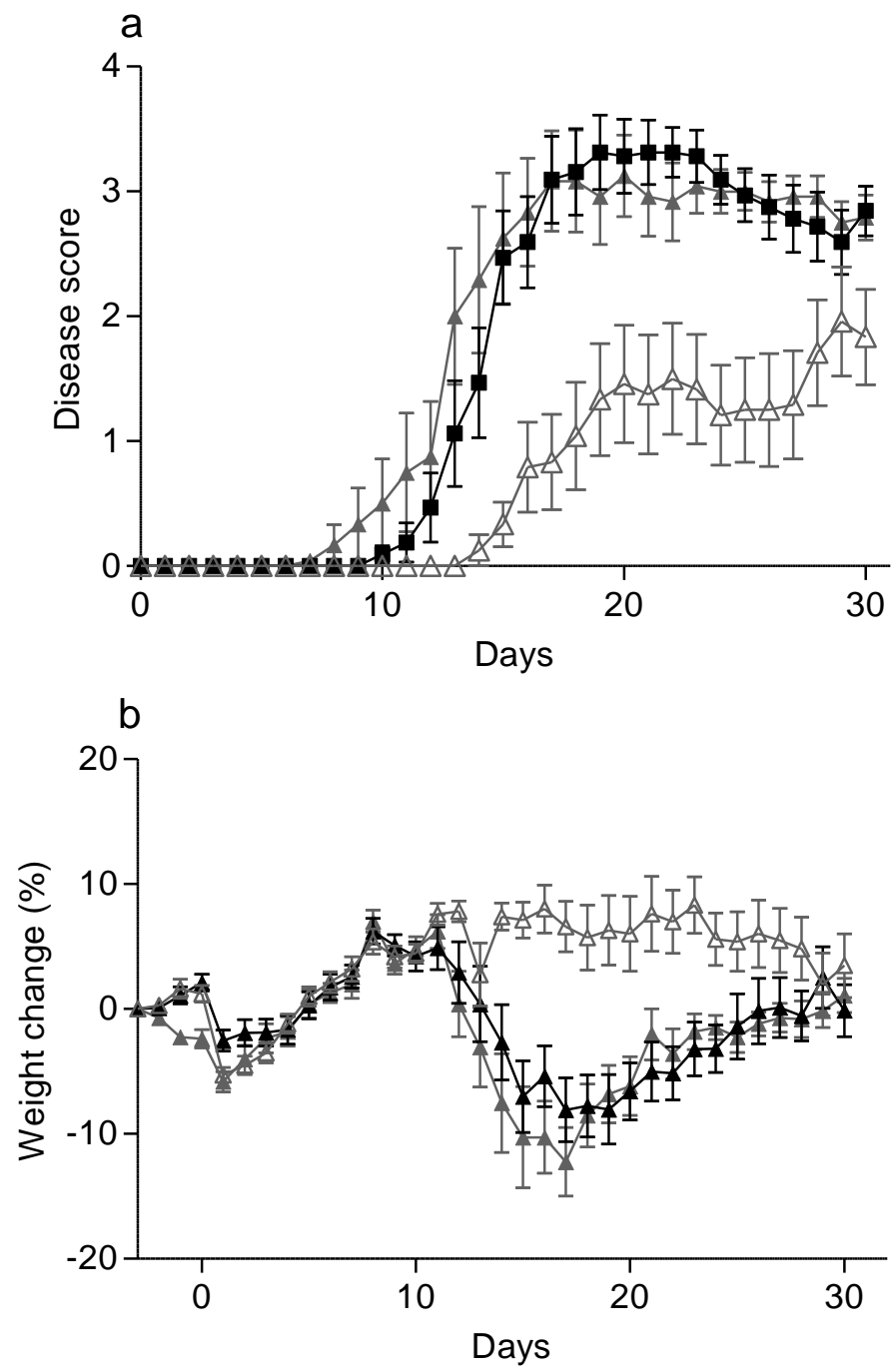

- Untreated

$\rightarrow$ MIS416 (d-3)

$\triangle$ MIS $416(\mathrm{~d} 0)$

Figure 7.1 MIS416 reduces EAE severity when administered on d0 p.i.

C57BL/6 mice were immunised for EAE and injected i.v. with $250 \mu \mathrm{l}$ saline vehicle (untreated) or with $250 \mu \mathrm{g}$ of MIS416 either 3 days before immunisation (d-3), or on the day of immunisation (d0). (a) Disease score of all mice. (b) \% weight change; calculated as \% change of individual mice from d0. Data represents two experiments $(n=12-16$ mice/group). Any mice that died were excluded from the analysis. 


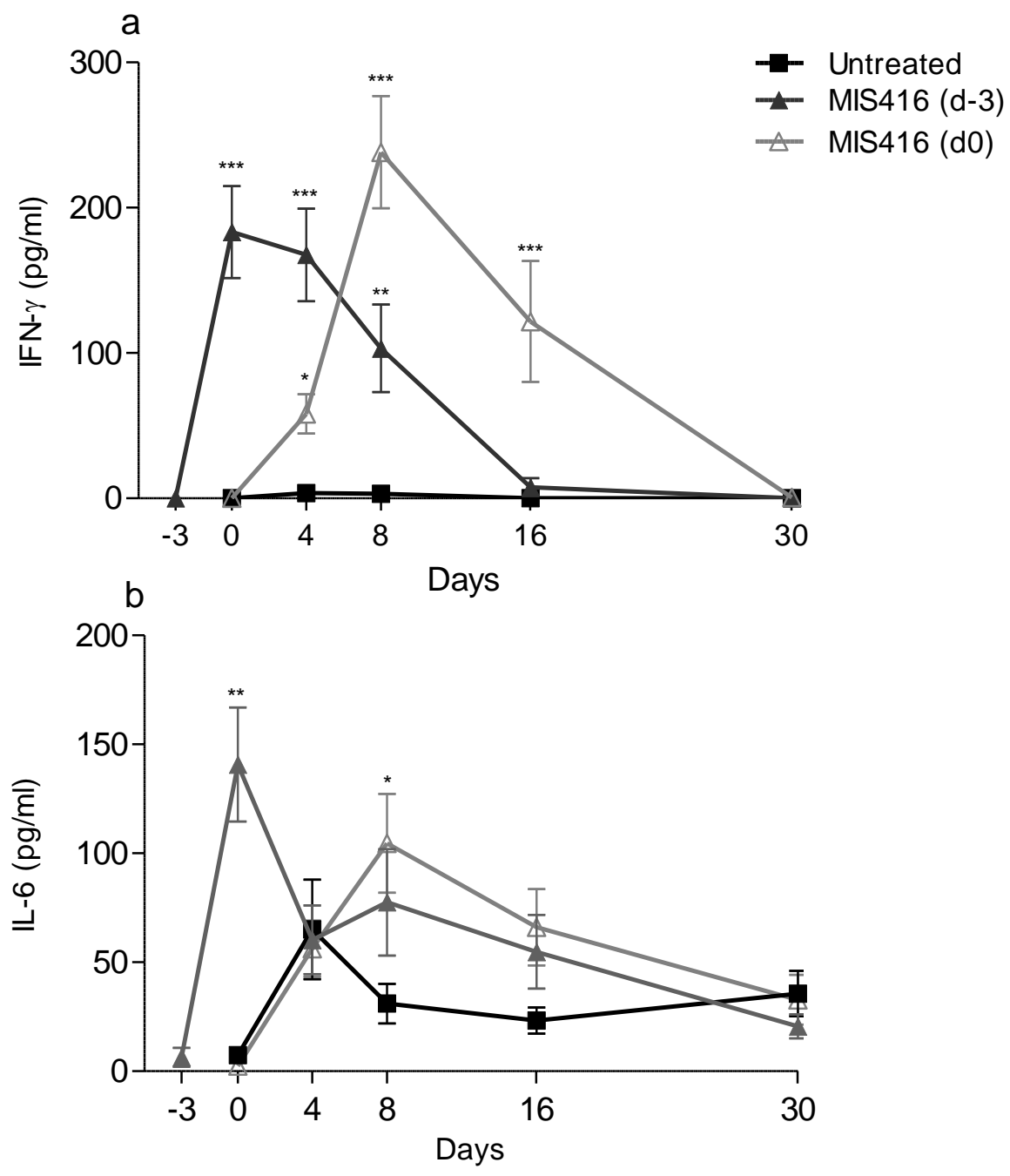

Figure 7.2 MIS416 increases serum IFN- $\gamma$ and IL-6 levels

C57BL/6 mice were immunised for EAE and left untreated or were treated with $250 \mu \mathrm{g}$ MIS416 either 3 days before immunisation (d-3), or on the day of immunisation (d0). Blood samples were taken on indicated days and cytokines within the serum of individual mice were analysed by CBA. (a) serum INF- $\gamma$ and (b) serum IL-6 concentrations. Data represents two experiments ( $\mathrm{n}=$ 12-16 mice/group). $* \mathrm{p}<0.05, * * \mathrm{p}<0.01$ or $* * * \mathrm{p}<0.001$ compared to immunised untreated mice, by Kruskal-Wallis test with Dunn's post hoc analysis. 


\subsubsection{Administration of MIS416 at disease onset reduces EAE disease severity}

To identify if MIS416 treatment would be effective if administered during or after disease onset a single $250 \mu \mathrm{g}$ dose of MIS416 was injected on either $\mathrm{d} 12$ or d20 p.i., respectively. Two days after administration of MIS416 at d12 p.i. disease symptom within these mice began to plateau and continued to exhibit a milder disease expression throughout the experiment, when compared to the untreated mice (Figure 7.3). However within 24 hours of the $\mathrm{d} 12$ p.i. MIS416 injection, $40 \%$ of the mice died (data not shown). A similar toxicity was observed in mice injected on d20 p.i., with $20 \%$ mortality occuring (data not shown). At either of the treatment time points the mice that survived exhibited no obvious toxicity, distress or weight loss (data not shown). No alteration in disease expression occurred in mice treated at d20 p.i., indicating that a single dose of MIS416 is effective during onset but not during the chronic phase of disease expression.

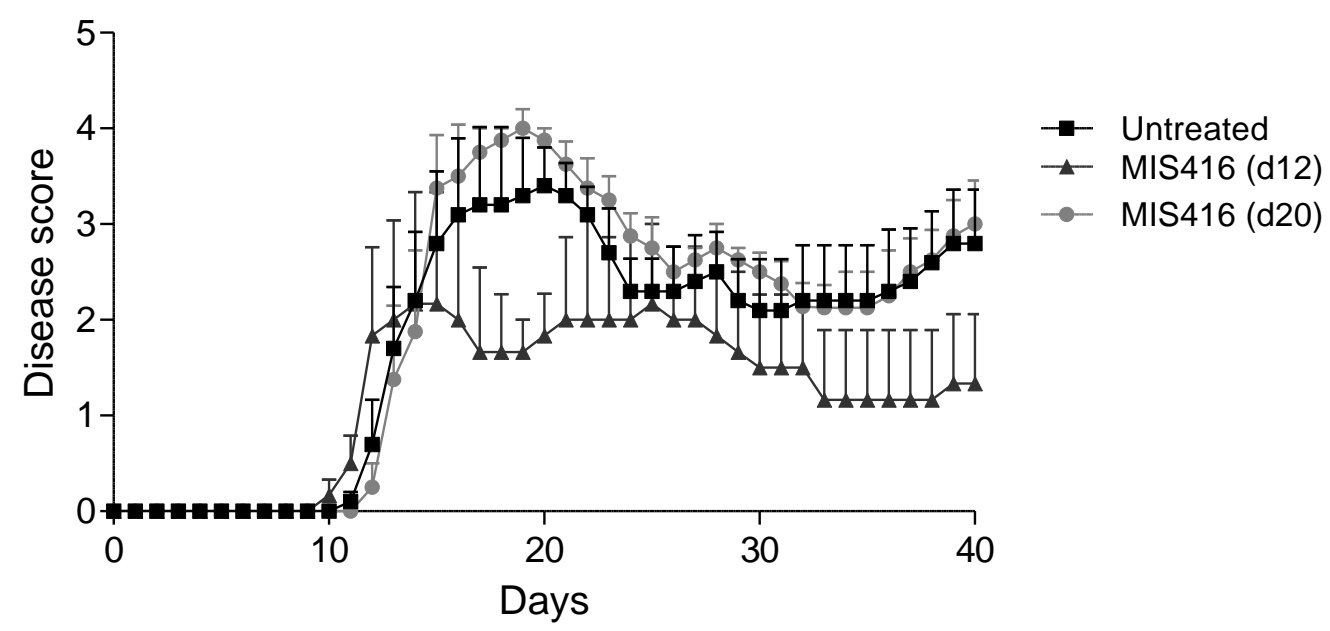

Figure 7.3 MIS416 reduces disease severity when administered on d12 p.i. but not d20 p.i. C57BL/6 mice were immunised for EAE and left untreated or were treated with $250 \mu \mathrm{g}$ MIS416 either 12 days after immunisation (d12) or 20 days after immunisation (d20). Data represents disease score of all live mice from one experiment $(n=3-5$ mice/group). Any mice that died were excluded from the analysis. 


\subsubsection{Weekly administration of MIS416 reduces EAE disease severity and alters cytokine production}

As it appeared that $250 \mu \mathrm{g}$ could be toxic if given during disease onset an alternate dosing strategy was employed. Mice were injected weekly with a lower dose of $100 \mu \mathrm{g}$ beginning at d0 p.i.. This approach proved to be an effective dosing strategy that resulted in long term reduction in disease severity (Figure 7.4a). Although one mouse died on d15 this appeared to be an isolated incidence as toxicity was not observed in any other mice (data not shown). Additionally subsequent studies using this treatment regime, to chronically treat over 30 mice, has not caused any mortality (personal communication; Anne La Flamme). Interestingly it appears that following MIS416 injection mice responded rapidly to the effects of MIS416 resulting in reduced disease scores. However this effect was transient as within 4-5 days post treatment, disease scores would often begin to increase. This trend can also be observed in the weight change of MIS416 treated mice (Figure 7.4b). These results suggest that MIS416, when administered at $100 \mu \mathrm{g}$, causes only a temporary alteration in diseases response and requires regular administration to effectively reduce disease burden.

To determine if repeated administration of lower doses of MIS416 altered serum cytokines, blood was collected 1 day post MIS416 treatment at various points over the duration of the experiment. Analysis of serum cytokines indicated that IFN- $\gamma$ production was significantly higher in MIS416 treated mice on d15 and d22 p.i.; however, by d65 p.i. no significant difference IFN- $\gamma$ production was detected (Figure 7.5a). Within MIS416 treated mice, IL6 production appeared higher at all 4 time points measured, however only d15 reached statistical significance (Figure 7.5b). 

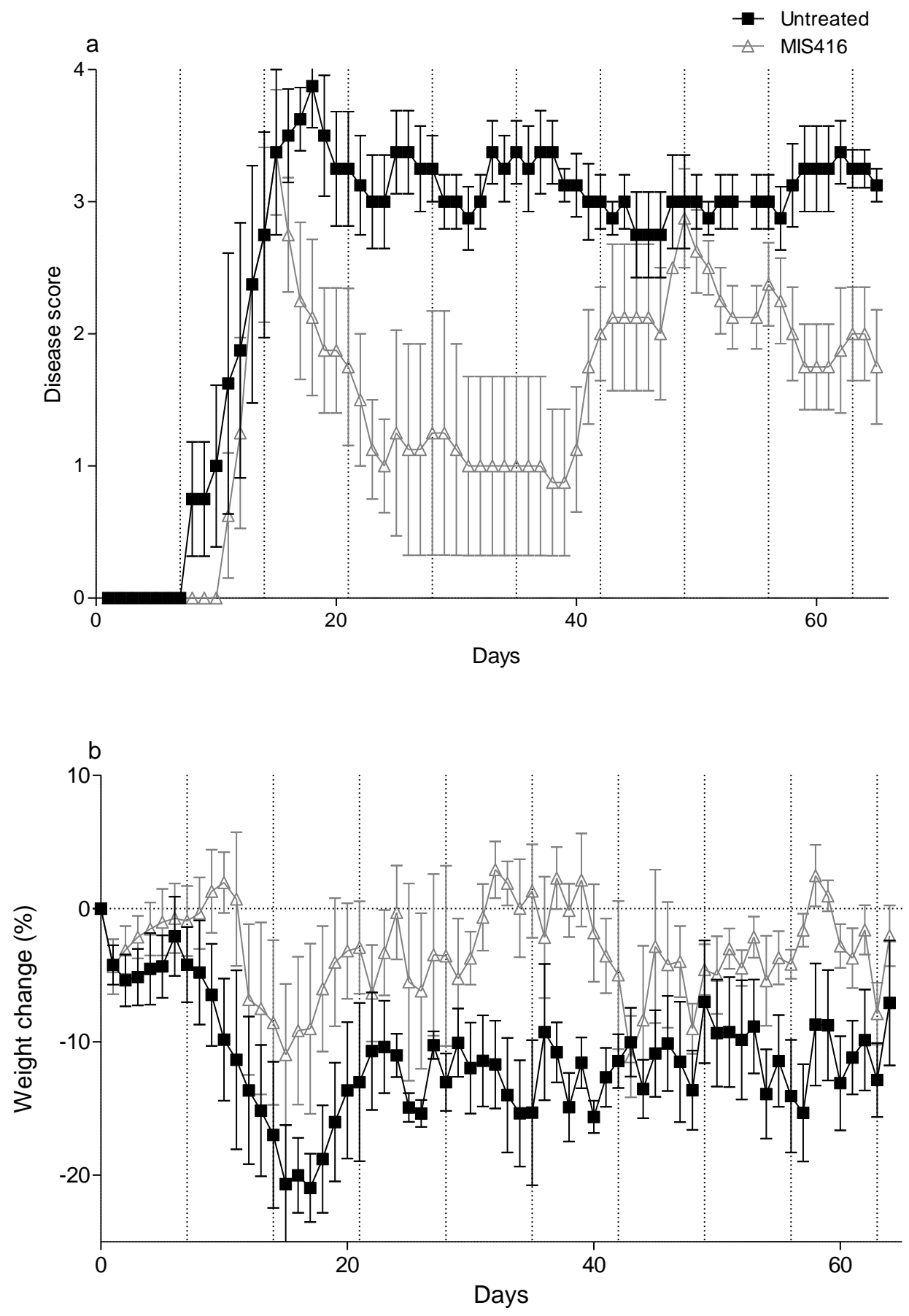

Figure 7.4 MIS416 Reduces disease severity when administered weekly at a low dose C57BL/6 mice were immunised for EAE and left untreated or were treated with one dose of $100 \mu \mathrm{g}$ MIS416 every 7 days for the duration of the experiment (dashed lines). (a) Disease score of sick mice and (b) \% weight change; calculated as \% change of individual mice from d0. Data represents one of two identical experiments $(n=4$ mice/group). Any mice that died were excluded from the analysis. 

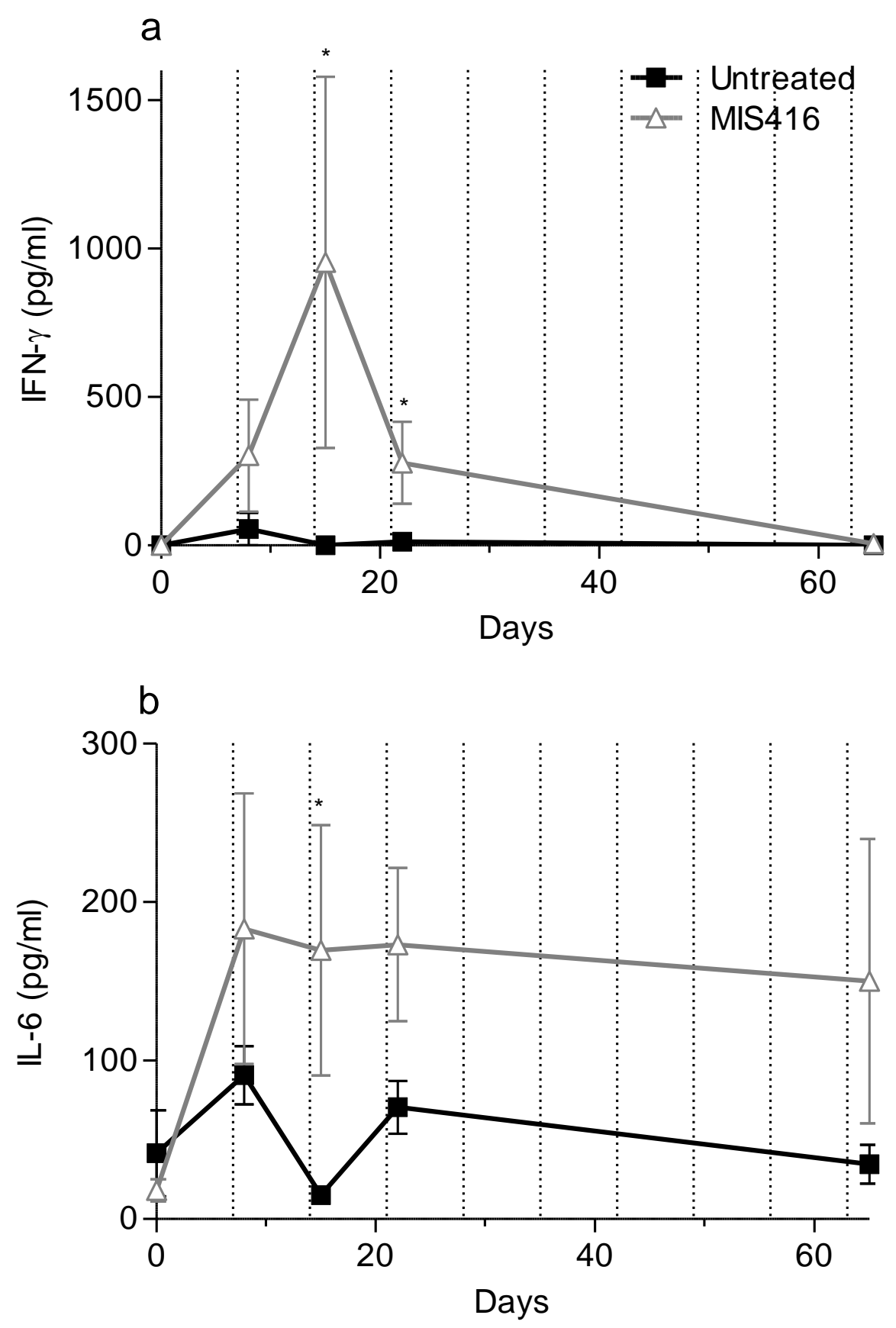

Figure 7.5 Weekly low dose MIS416 increases serum IFN- $\gamma$ during disease onset

C57BL/6 mice were immunised for EAE and left untreated or were treated with one dose of $100 \mu \mathrm{g}$ MIS416 every 7 days for the duration of the experiment (dashed lines). Blood samples were taken on days $0,8,15,22$ and 65 p.i. and cytokines within the serum of individual mice were analysed by CBA. (a) serum IFN- $\gamma$ and (b) serum IL-6 concentrations. Data represents one of two identical experiments (n $=4$ mice/group). $* \mathrm{p}<0.05$ compared to immunised untreated mice, by a Mann Whitney test. 


\subsubsection{IFN- $\gamma$ signalling is necessary for MIS416 induced disease modification}

As large increases in IFN- $\gamma$ were detected within the serum of MIS416 treated mice during disease onset, it was speculated that this increase could be associated with the disease modifying effects of MIS416. To investigate if IFN- $\gamma$ had a role in MIS416 disease protection, IFN- $\gamma$ was depleted during the induction phase of EAE. To do this, groups of mice were treated with MIS416 on d0 p.i. or left untreated, then on day 3 and 7 p.i. mice were either injected with an anti-IFN- $\gamma$ antibody (clone: AN18) or an equal concentration of non-specific rat-IgG.

Disease symptoms and peak disease severity within untreated AN18-injected mice developed earlier than other treatment groups, indicating that AN18 treatment was altering disease expression (Figure 7.6). In comparison MIS416-treated, AN18-injected mice had a reduced disease course. Unfortunately within this experiment, administration of MIS416 on d0 p.i. did not result in a reduction in disease expression in mice not receiving AN18 making it difficult to ascertain if administration of AN18 had any effect on MIS416 disease modifying activity. Interestingly when IFN- $\gamma$ production in the serum was analysed very high levels of IFN- $\gamma$ were detected in both AN18 treated groups (Figure 7.7). Whether these high levels of IFN- $\gamma$ were the result of increased cytokine production (due to a lack of negative feedback) or the result of AN18 inhibiting IFN- $\gamma$ receptor binding (thus inhibiting cellular uptake) is not known.

To overcome the potential confounding factors that occurred with antibody depletion of IFN- $\gamma$, an alternative strategy using IFN- $\gamma$-deficient mice was used to explore the potential role of IFN- $\gamma$ in MIS416-induced disease modification. Additionally, as the d0 p.i. treatment had been unsuccessful in the previous experiment, the more effective weekly dosing schedule of $100 \mu \mathrm{g}$ MIS416 was used to ensure effective disease modification. This treatment resulted in a typical reduction in disease severity within WT mice compared to untreated mice (Figure 7.8). In contrast, IFN- $\gamma$-deficient mice treated with MIS416 developed severe disease similar to that of untreated IFN- $\gamma$-deficient mice (Figure 7.8). This result indicates that IFN- $\gamma$ is necessary for effective disease modification. 

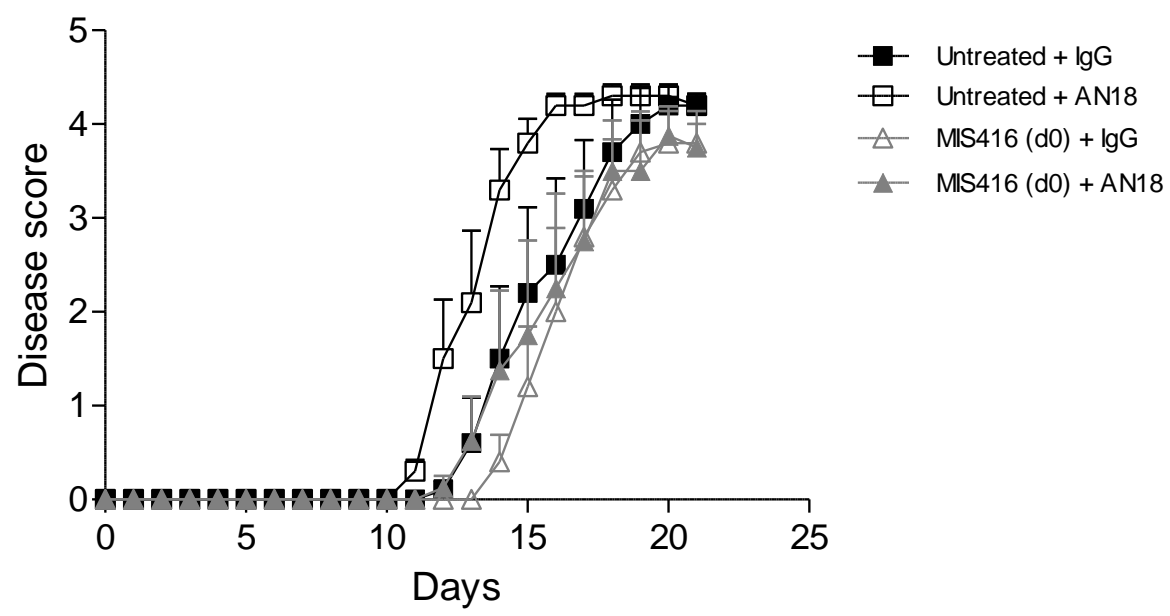

Figure 7.6 Administration of anti-IFN- $\gamma$ antibody does not alter the disease course in MIS416 treated mice

C57BL/6 mice were immunised for EAE and left untreated or were treated with $250 \mu \mathrm{g}$ MIS416 on

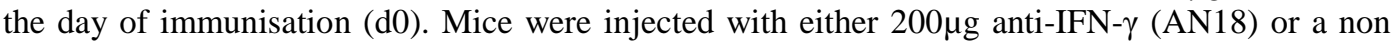
specific rat $\operatorname{IgG}(\mathrm{IgG})$ on days 4 and 7. (a) Disease score of sick mice. Data represents one experiment ( $\mathrm{n}=5$ mice/group).

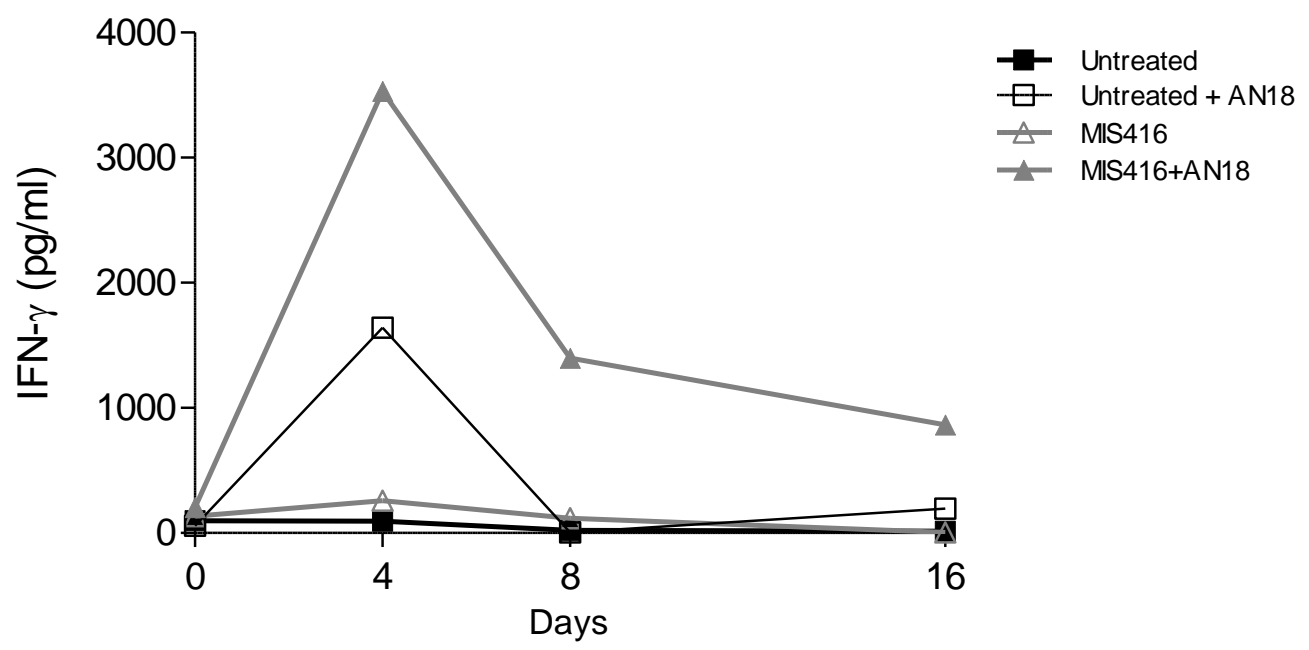

Figure 7.7 Administration of anti-IFN- $\gamma$ antibodies causes increased detection of IFN $\gamma$ C57BL/6 mice were immunised for EAE and left untreated or were treated with $250 \mu \mathrm{g}$ MIS416 on the day of immunisation (d0). Mice were injected with either $200 \mu \mathrm{g}$ anti-IFN- $\gamma$ (AN18) or a nonspecific rat $\operatorname{IgG}(\operatorname{IgG})$ on days 3 and 7. Blood samples were taken and on indicated days and IFN- $\gamma$ concentrations within pooled serum of mice from each group was determined by CBA. Data represents one experiment ( $\mathrm{n}=5$ mice/group). 


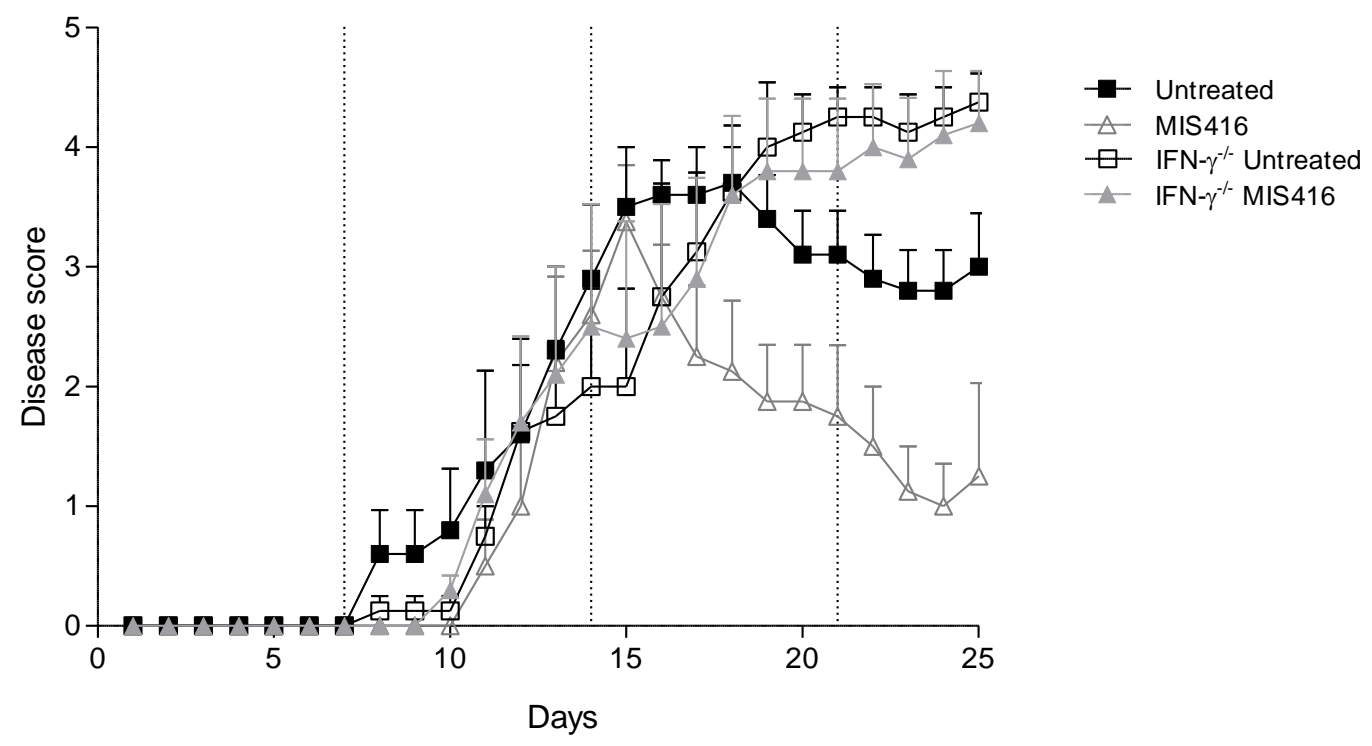

Figure 7.8 MIS416 does not reduce disease severity in IFN- $\gamma$ deficient mice

C57BL/6 or C57BL/6-ifngtm1Ts (IFN- $\gamma$ deficient) mice were immunised for EAE and left untreated or were treated with one dose of $100 \mu \mathrm{g}$ MIS416 every 7 days for the duration of the experiment (dashed lines). Data represents one experiment $(n=4-5$ mice/group). 


\subsection{Discussion}

This pilot study was conducted to determine if MIS416, a promising new MS therapeutic, was able to modify disease in the EAE model of MS and accordingly identify if the EAE model could be used to understand the mechanism by which MIS416 is protective. The results from the current study indicate that MIS416 effectively reduces disease severity when administered immediately before EAE immunisation or during disease onset. Furthermore, the disease modifying effects of MIS416 appear to be related to IFN- $\gamma$ as MIS416 failed to protect IFN- $\gamma$ deficient mice from normal disease development. Thus, EAE proved to be a valuable tool to further study MIS416 and to investigate potential mechanisms of action.

Analysis of cytokines from d-3 and d0 p.i. treated mice revealed that MIS416 induced increased secretion of IL-6 and IFN- $\gamma$. Elevations in these cytokines indicate that MIS416 was inducing a characteristic inflammatory response, as is observed after TLR9 or NOD2 signalling (Girvan et al., 2010; Magalhaes et al., 2011). A difference in the kinetics of cytokine production occurred between the d-3 and d0 p.i. treatment groups, with peak levels of IL- 6 and IFN- $\gamma$ occurring at $\mathrm{d} 0$ and $\mathrm{d} 8$ respectively. Given disease protection was observed in the d0 but not d-3 p.i. treated mice, it was speculated that the peak in cytokine production at $\mathrm{d} 8$ and the sustained IFN- $\gamma$ at $\mathrm{d} 16$ p.i. might be associated with disease protection in d0 MIS416 treated mice, as these time points coincided with the induction phase of the disease process.

Most of the literature investigating the role of IL-6 in the context of EAE has implicated this cytokine in the disease process (Mendel et al., 1998; Okuda et al., 1999; Serada et al., 2008). In contrast, IFN- $\gamma$ has been demonstrated to have a paradoxical role in immune regulation, despite its classical pro-inflammatory actions (Zhang, 2007). Therefore, it was considered more likely that IFN- $\gamma$ rather than IL-6 would be associated with the protective effects of MIS416. Indeed when IFN- $\gamma$ deficient mice were injected once a week with 100 $\mu \mathrm{g}$ of MIS416 no protection in disease expression was observed despite this treatment regime inducing a substantial reduction in disease severity in IFN- $\gamma$ sufficient WT mice. 
This result clearly indicates that IFN- $\gamma$ is necessary factor in MIS416-induced disease protection.

Although IFN- $\gamma$-producing Th1 cell have typically been associated with EAE disease process, a regulatory role for IFN- $\gamma$ in EAE is also evident. It is well documented EAE is exacerbated in either IFN- $\gamma$-deficient and IFN- $\gamma$ receptor-deficient mice (Billiau et al., 1988; Ferber et al., 1996b; Sabatino et al., 2008). IFN- $\gamma$ is also protective in EAE when exogenous IFN- $\gamma$ is administered or if endogenous IFN- $\gamma$ production is enhanced (Furlan $e t$ al., 2001; Gran et al., 2004; Voorthuis et al., 1990). Additionally, it has been demonstrated that certain myelin antigens, which are poorly encphalogenic, inhibit disease expression due to the induction of high levels of IFN- $\gamma$ (Minguela et al., 2007; Sabatino et al., 2008). Collectively, these studies highlight the potential regulatory role IFN- $\gamma$ can have on EAE disease expression.

There are many potential ways IFN $-\gamma$ could induce regulatory processes or protective effects in EAE. IFN- $\gamma$ can induce macrophages and microglia to secrete $\mathrm{T}$ cell antiproliferative or pro-apoptotic factors, for example through up-regulation of iNOS or IDO production (Kwidzinski et al., 2005; Willenborg et al., 1999). Sustained levels of IFN- $\gamma$ can also directly reduce $\mathrm{T}$ cell reactivity though alteration of receptor expression (Bronstein-Sitton et al., 2003) IFN- $\gamma$ production may also counter regulate Th17 response resulting in a biasing of encephalogenic responses towards Th1 (Berghmans et al., 2011; Murphy et al., 2010). Attenuation of tissue destruction can also be induced though IFN- $\gamma$ mediated down-regulation of MMP production (Ho et al., 2008; Hu et al., 2009). It is conceivable that one or multiple IFN- $\gamma$-induced regulatory mechanisms are associated with MIS416 disease modifying effects.

In contrast to the conclusive results observed with IFN- $\gamma$-deficient mice, antibody depletion of IFN- $\gamma$ failed to provide any definitive results. This failure is likely due to two confounding factors that are discussed below; 1) the lack of disease protection normally induced by MIS416 treatment and 2) the possibility that administration of AN18 (anti-IFN$\gamma$ antibody) did not effectively deplete IFN- $\gamma$. 
In the experiment using AN18, MIS416 was administered as a single $250 \mu \mathrm{g}$ dose at d0 p.i. and uncharacteristically, no protection from disease was observed. Interestingly, however, the levels of IFN- $\gamma$ in MS416 treated mice peaked early (at d4 p.i.) and returned to basal levels by d8 p.i. (Figure 7.7), which is in contrast to the previous experiments, that demonstrated MIS416 administered on d0 p.i. caused a sustained elevations in IFN- $\gamma$ until d16 p.i. (Figure 7.2). It is possible that the lack of disease protection could be related to the alteration in IFN- $\gamma$ concentration kinetics observed in this particular experiment.

A slight delay in disease onset did however occur in MIS416 treated AN18 injected mice when compared to AN18 injected-untreated mice. A substantial increase in IFN- $\gamma$ was detected in AN18 treatment groups particularly in MIS416 treated mice. It was not determined if these increases were due exclusively to AN18 bound IFN- $\gamma$ or if unbound IFN- $\gamma$ also contributed to the measured increase. Given the high levels of IFN- $\gamma$ detected in AN18-injected mice, there is the potential that the binding capacity of AN18 to IFN- $\gamma$ became saturated and thus unbound IFN- $\gamma$ was present. Previous studies that have used anti-IFN- $\gamma$ antibody in the context of EAE have used higher concentration of antibody (i.e. $500 \mu \mathrm{g}-2 \mathrm{mg}$ per mouse) to achieve IFN- $\gamma$ depletion (Duong et al., 1994b; Espejo et al., 2001; Voorthuis et al., 1990). Therefore, it is possible that the $250 \mu \mathrm{g}$ dose used in the current study was not large enough to fully deplete IFN- $\gamma$. If unbound IFN- $\gamma$ was present, it is possible that the slight delay in disease expression of MIS416 treated AN18 injected mice could be due to the increased levels of IFN- $\gamma$. Collectively, these results indicate that antibody depletion of IFN- $\gamma$, using this protocol, cannot provide an accurate indication of the role IFN- $\gamma$ has in MIS416 disease modifying effects. Despite this, given the results within IFN- $\gamma$ deficient mice, it can be concluded that IFN- $\gamma$ signalling is necessary for the disease suppression induced by MIS416.

When $250 \mu \mathrm{g}$ MIS416 was administered at d12 p.i. $40 \%$ of the mice died while the same dose, administered at d20 p.i. $20 \%$ of mice died. In contrast, mice administration of $250 \mu \mathrm{g}$ MIS416 on d0 p.i. was well tolerated. This may suggest that the toxicity observed at d12p.i. and $\mathrm{d} 20$ p.i. was related to EAE disease kinetics and is supported by a previous study demonstrating that MIS416 is well tolerated in mice with no clinical abnormalities or 
mortality being observed in doses as high as $900 \mu \mathrm{g}$ (Girvan et al., 2010). It is likely that the toxicity observed following EAE immunisation was due to the combination of EAE induced inflammatory response and the induction of cytokine production by MIS416, which may have resulted in a 'cytokine storm' and systemic shock. Such an amplified response could be due to cytokine priming which results in enhanced TLR signal transduction increased TLR expression as well as inactivation of feedback inhibition pathways (Hu et al., 2009). The observed toxicity indicates that high doses (i.e. $250 \mu \mathrm{g}$ ) of MIS416 should not be administered during EAE onset.

Administration of $100 \mu \mathrm{g}$ MIS416 every 7 days proved an effective and well tolerated treatment regime, which resulted in reduced disease scores sustained over the duration of the experiment. When looking at the disease score of MIS416-treated mice in relation to the timing of the weekly dosing an interesting trend appears that is particularly apparent in the chronic phase of disease (d40-d65 p.i.). Within a day or two after MIS416 administration average disease scores of treated mice reduced or plateaued, while in the days proceeding the subsequent treatment disease scores began to increase. This pattern indicates a number of things: 1) MIS416 is having an active role in disease suppression even during the chronic phase of disease, 2) the disease modifying effects of MIS416 are

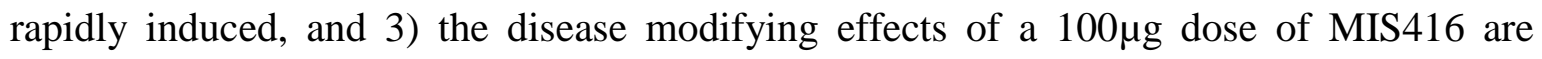
transient and begin to diminish within 4-5 days of administration.

It also appears that the effects of MIS416 are most pronounced during the earlier stages of disease. This time in the disease process is when increased IFN- $\gamma$ levels were detected. As IFN- $\gamma$ was demonstrated to have a role in MIS416 mechanism of action, it is conceivable that the dramatic suppression of disease following the administration of MIS416 on d14 p.i. is correlated to the large increase of IFN- $\gamma$ detected within the serum of MIS416 treated mice on $\mathrm{d} 15$ p.i. If the large increase in IFN- $\gamma$ induced by MIS416 is correlated to the disease suppression during the early stages of disease, it is interesting to note that no increase in IFN- $\gamma$ was detected in the serum of MIS416 treated mice by d65 p.i. This may indicate that during the chronic phases of disease, the effects of MIS416 on disease are not dependent on IFN- $\gamma$ but some other factor. Alternatively, it is possible that only low levels 
of IFN- $\gamma$ are induced during the chronic phase of disease, thus no systemic increase (i.e. within the blood) would be detectable.

A potential disease modifying factor that was not explored in this pilot study is the production of type I interferons. Previous studies have shown TLRs and specifically MIS416 can induce type I interferon production (Girvan et al., 2010; O'Brien et al., 2010; Touil et al., 2006). Administration or production of IFN- $\beta$ (a type I interferon) can reduce EAE severity (Floris et al., 2002; Galligan et al., 2010; Yu et al., 1996). Additionally there can be considerable cross talk between type I and type II interferon signalling pathways as IFN- $\gamma$ can induce type I secretion and vice versa (Schroder et al., 2004). Interestingly a recent study found that the disease modifying effects of IFN- $\beta$ were dependent on IFN- $\gamma$ signalling (Axtell et al., 2010). Collectively these results suggest that some of the disease modifying effects induced by MIS416 could be mediated through IFN- $\beta$ production, and any future studies into the effect of MIS416 in EAE should explore this possibility.

\subsection{Summary}

The current study has demonstrated that MIS416 is effective for the treatment of EAE and has provided evidence for the function of IFN- $\gamma$ in MIS416 disease modification. How IFN- $\gamma$ might mediate these effects and if other soluble factors are involved in this process remains to be determined. Additionally the effects of MIS416 on cellularity and CNS pathology needs to be explored in future experiments. Overall, the finding from the current study provides a useful foundation from which further in-depth studies can be conducted. 


\section{Chapter 8:}

\section{General Discussion}




\subsection{Overview}

Based on the work conducted in this thesis a set of hypothesised mechanisms with which MSDs, risperidone and MIS416 could alter EAE disease expression have been summarised below (Figure 8.1). A more in-depth discussion of the main findings from this thesis, the potential clinical implications, and possible future directions are addressed individually for each drug in the rest of this chapter. 


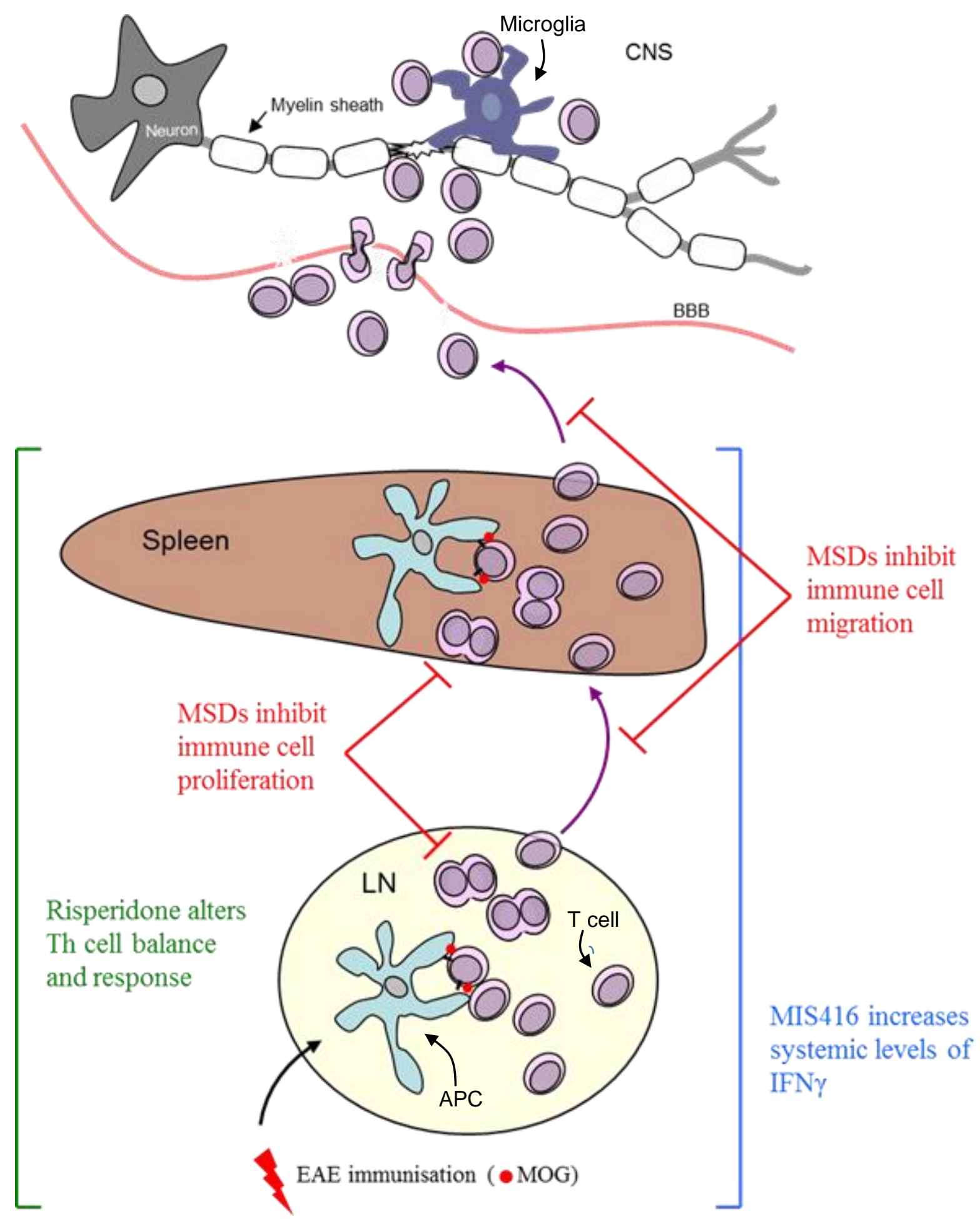

\section{Figure 8.1 How MSDs risperidone or MIS416 could potentially alter the EAE disease pathogenesis}

Following immunisation for EAE antigen presenting cells (APCs) within the draining LN present the myelin antigen (eg. MOG) along with co-stimulatory molecules to naïve T cells. Once activated these autoreactive T cells release inflammatory cytokines, proliferate, and migrate to the spleen or to the CNS. Following further encounters with APCs, autoreactive T cells within the spleen release inflammatory cytokines and proliferate, further amplifying the inflammatory process. Autoreactive T cells from the spleen and LN migrate across the blood brain barrier (BBB), and encounter myelin antigens presented within the CNS by resident microglia or infiltrating APCs. This process leads to the induction of a CNS based inflammatory response and subsequent damage to myelin. Shown are hypothesised mechanisms with which MSDs, risperidone or MIS416, could alter disease pathogenesis, based on the research within this thesis. 


\subsection{Microtubule-stabilising drugs}

\subsubsection{Summary}

The current study demonstrated that MSDs are capable of delaying EAE onset and altering disease progression although high concentrations and prolonged exposure were necessary for MSDs to be effective. All three MSD compounds tested (PTX, DTX and IXB) exhibited disease modifying activity, suggesting that a common underlying mechanism of action may be responsible for these effects. Explorations into possible mechanisms of action found that PTX both inhibited immune cell proliferation as well as CNS infiltration. The relative contribution of these two factors in the overall disease modifying effect may be dependent on the timing of administration, with the anti-proliferative actions of PTX having a more significant role in early treatment; while the effects of PTX treatment at later time points may be more dependent on inhibition of immune cell migration. Finally, studies into potential synergy revealed that a combination of the MSDs, Pel A and IXB can act synergistically to inhibit EAE; as can PTX when combined with the commonly used MS drug, GA.

\subsubsection{Clinical implications}

In 2002, a Phase II clinical trial tested the efficacy of PTX for the treatment of secondary progressive MS. Patients in this trial were administered either a placebo or $50 \mathrm{mg} / \mathrm{m}^{2}$ PTX (1 dose/month for 6 months) (Angiotech-Pharmaceuticals, 2002). No significant reduction in disease parameters was observed between the treatment and placebo groups. Given the results from the current study, it is not surprising that PTX when administered 1 dose/month had little effect on disease. In the current study efficacy of MSD for the treatment of EAE was only exhibited when MSDs were administered at high doses over a number of days. To translate these disease modifying effects into a clinical setting, administration of PTX, or any other MSD, would have to be prolonged either by using a multi-day infusion or administering multiple doses in close temporal proximity. A high dose prolonged exposure treatment is a feasible option, as 96 hour infusions of $140 \mathrm{mg} / \mathrm{m}^{2}$ PTX are already used for treatment of metastatic cancers (Moulder et al., 2010; West et al., 2005). Likewise a phase I clinical trial has demonstrated that a continuous 10 day infusion of $21 \mathrm{mg} / \mathrm{m}^{2} /$ day can be administered safely (Shade et al., 1998). As a $20 \mathrm{mg} / \mathrm{kg}$ dose in 
mice roughly equates to a $60 \mathrm{mg} / \mathrm{m}^{2}$ within humans, the dose and treatment regimes used within the current study are applicable to a clinical setting (Denardo et al., 1997).

In the current study, IXB was found to exhibit a small therapeutic advantage in modifying EAE expression over PTX or DTX. Therefore, if future clinical trials were to explore the use of MSDs to treat MS, IXB may prove most beneficial. Additionally, the finding that combined administration of Pel A and IXB results in a synergistic inhibition of EAE highlights the potential for enhancing therapeutic effects of MSDs though synergistic combinations. Although toxicity was observed in some mice treated with synergistic doses of Pel A and IXB, this toxicity is likely to be related to an interaction with EAE specific disease processes. Previous research has demonstrated that EAE immunised mice are acutely more sensitive to MSD induced toxicity than unimmunised mice (Crume et al., 2009). Therefore, it is conceivable that the toxicity observed in some mice treated with synergistic combinations of Pel A and IXB may only occur in the context of EAE. However, the possibility that this toxicity could also occur if used clinically to treat MS cannot be discounted. Therefore, further experimentation into the exact cause of toxicity or identification of less toxic synergistic combinations of MSDs is necessary, before considering using such a treatment in a clinical setting.

Assuming further research does indicate that the toxicity observed in the current study was is specific to the context of EAE, synergistic combinations of Pel A and IXB in a clinical setting may prove effective for either treating MS or for use as a cancer chemotherapeutic. Although the current study has demonstrated the synergistic effects of Pel A and IXB only within EAE, the benefits of this treatment regime are also relevant to cancer research. Most MSD based cancer chemotherapies have been developed based on their anti-proliferative actions. The current study has demonstrated that the disease modifying actions of MSDs in EAE are likely to be caused by inhibition of migration and proliferation. Inhibition of tumour growth and metastasis presumably also occurs though these same mechanisms.

A limiting factor in the development of Pel A as a clinical chemotherapeutic is the supply of Pel A at the quantities needed (personal communication, John H Miller). The impact of 
this limitation could be partially resolved if Pel A were used in a synergistic combination with IXB; as the total amount of Pel A required would be reduced. Additionally if Pel A has the potential to provide benefits in two diseases, there is a greater likelihood that the necessary funding could be obtained to synthesise clinically relevant quantities of Pel A.

The most practical and effective way to use MSDs as a treatment for MS may be to administer a MSD as a brief pulse (eg a high dose over 3 days), then follow this treatment with a chronically administered immune modulating drug such as GA. The current study demonstrated that treating mice with PTX followed by a week of GA treatment proved to be effective at limiting long-term disease severity even though both PTX and GA were only administered for a short period of time. A number of preliminary clinical studies on relapsing-remitting MS patents have shown that mitoxantrone followed by GA treatment is more effective than either treatment alone (Arnold et al., 2008; Ramtahal et al., 2006; Vollmer et al., 2008). In fact this combined treatment has recently been patented by the makers of GA, indicating a perceived potential of this treatment ${ }^{4}$. However, as mitoxantrone has a maximal lifetime dose and causes considerable and irreversible side effects, the use of this combination is likely to be limited (Neuhaus et al., 2006). Given the combined effects of PTX and GA in altering EAE disease expression observed in the current study, further experiments into the efficacy of these compounds in combination may provide an effective treatment alternative.

\subsubsection{Future directions}

The current study identified that inhibition of immune cell migration and proliferation occurs following in vivo administration of PTX. To what extent each of these two mechanisms of actions contributes to alterations in the EAE disease response remains to be determined. Treatment with PTX immediately after immunisation inhibited immune cell proliferation and subsequently also inhibited encephalogenic $\mathrm{T}$ cell responses, indicating that the anti-mitotic actions of a MSD has a crucial role inhibiting the disease process when administered immediately after immunisation. The importance of these anti-mitotic

\footnotetext{
${ }^{4}$ http://www.freepatentsonline.com/7968511.html
} 
actions, when drug is administered at a later time point, is not so clear. To identify specifically if MSDs are still inhibiting immune cell proliferation when administered at later time points in the disease process an experiment using BrdU incorporation could be conducted. BrdU incorporates into the DNA of dividing cells and can be detected by flow cytometry. Therefore, pulsing mice with BrdU at times points of interest (eg. d6-12 p.i.) would provide a snapshot of any cell proliferation occurring. MSD treated and untreated mice could then be compared to specifically identify differences in proliferation.

Immune cell proliferation was found to be completely inhibited in the spleens and blood but only attenuated in the dLN of PTX-treated mice. This result is likely to indicate that higher concentrations of PTX are achieved in the spleen and blood compared to the dLN. To test this hypothesis, HPLC could be used to measure specific concentrations of PTX within the different tissues. Alternatively, fluorescently labelled PTX (eg. Flutax) could be administered to mice and the florescent intensity within the different tissue could be used as a relative indicator of of PTX distribution.

Adoptive transfer experiments may prove valuable in teasing out the specific mechanisms by which MSDs inhibit disease expression. Induction of passive EAE could be compared between mice that received adoptively transferred $\mathrm{T}$ cells isolated from MSD-treated immunised mice and those that received $\mathrm{T}$ cells from immunised, untreated mice. If $\mathrm{T}$ cells from MSD-treated, immunised mice induced the same EAE disease response as $\mathrm{T}$ cells from immunised, untreated mice, it could be concluded that alterations in $\mathrm{T}$ cell encephalitogenic responses were not the cause of the disease modification by MSDs. Additionally this result would support the hypothesis that inhibition of migration is a more important factor in MSD disease modification than reduced proliferation of T cells.

Although the current study demonstrated that in vivo administration of PTX inhibited immune cell migration, it still remains to be determined how this inhibition is occurring specifically. This question could be investigated directly in vivo using two photon microscopy (Kreisel et al., 2010; Matheu et al., 2011). Alternately, changes in migratory 
ability could be indirectly analysed by isolation of macrophages or $\mathrm{T}$ cells following in vivo MSD administration and exploring migratory capacity using ex vivo migration assays.

The current study is the first to demonstrate the potential for using MSDs in synergistic combinations to treatment an inflammatory disease. Further research should be conducted to investigate potential synergy between combinations of Pel A and IXB to identify if lower doses can be as effective without inducing toxicity. Additionally it would be interesting to explore the potential of these synergistic combinations as a treatment for other MSD-responsive diseases, such as cancer and vascular restinosis (Scheller et al., 2006; Tepe et al., 2008). The current study also demonstrated the exciting potential of using MSDs to enhance the disease modifying effects of GA. The use of MSDs in combination with immune modulating drugs, such as GA, should be explored further. Optimisation of a combined MSD and GA treatment could be conducted, as well as exploring the potential of MSDs to enhance other currently used MS therapeutics.

\subsection{Risperidone}

\subsubsection{Summary}

The current study demonstrated that risperidone could reduce EAE disease burden and severity when administered daily at a clinically relevant dose. The reduction in disease severity was accompanied by increased proportion of splenic $\mathrm{CD}^{+} \mathrm{T}$ cells and increased splenocyte antigen-specific IFN- $\gamma$ production. Additionally, risperidone treatment of stimulated bone marrow-derived macrophages resulted in a reduction in IL-12, an increase in IL-10 cytokine production, as well as reduced expression of the co-stimulatory molecule CD40. These findings indicate that risperidone can induce an anti-inflammatory phenotype within in vitro cultured macrophages. Collectively the results from these experiments indicate that risperidone can alter inflammatory immune responses and highlight the potential value of developing risperidone as a treatment for MS. 


\subsubsection{Clinical implications}

The current study demonstrated that risperidone reduces EAE disease severity and raised the interesting possibility that this antipsychotic could be used to treat an inflammatory disease such as MS. Accounting for differences in pharmacokinetics between mice and humans the effective concentrations with which risperidone attenuated EAE in the current study is comparable to doses currently used clinically for the treatment of schizophrenia (Kapur et al., 2003; Karl et al., 2006; Naiker et al., 2006). At these concentrations, risperidone is well tolerated and side effects relatively minor.

Assuming the immunomodulating effects of risperidone in EAE translate into similar effects within MS, there is potential that risperidone could be beneficial if co-administered with the current MS treatment, natalizumab. One of the limitations of natalizumab therapy as an MS treatment is the increased risk of lethal progressive multifocal leukoencephalopathy (PML) (Chaudhuri, 2005; Tan et al., 2010). PML is caused by the JC virus, and there is evidence suggesting that this virus infects neural cells via the $5-\mathrm{HT}_{2 \mathrm{a}}$ receptor and inhibiting these receptors may reduce JCV infection (Elphick et al., 2004; Maginnis et al., 2010). There is, however, still some debate as to the specificity of this infection route, as non-5- $\mathrm{HT}_{2 \mathrm{a}}$ expressing cells also can be infected with the virus in vitro (Chapagain et al., 2007). Despite this caveat, studies have shown that 5-HT2a receptor antagonists, including risperidone, can reduce or inhibit JCV infection although the effect may be strain dependent (Chapagain et al., 2008; Elphick et al., 2004; Nukuzuma et al., 2009; O'Hara et al., 2008). There are also case reports of patients recovering from PML following risperidone treatment (Focosi et al., 2007; Kast et al., 2007). While case reports cannot provide a causal link between risperidone treatment and recovery from PML, combined with cell-based studies, these reports raise an interesting possibility that risperidone may confer some benefit in reducing JCV infection. If risperidone can reduce JVC infection, co-administration of natalizumab with risperidone may have an additive or synergistic effect at reducing MS disease symptoms with the additional benefit of reducing chances of contracting PML. 
Risperidone can be administered orally or though slow release depot injections and has far less severe side effects than all currently available MS treatments. Indeed studies in healthy individuals, that do not suffer from any mental illness, have shown the most common side effects from acute administration of risperidone (drowsiness or headaches) are relatively minor (Artaloytia et al., 2006; Van Schaick et al., 2003). Collectively, it appears that risperidone is relatively well tolerated both within healthy individuals and when used as an antipsychotic (Artaloytia et al., 2006; Belotto et al., 2010; Corbeil et al., 2011; Leucht et al., 1999; Van Schaick et al., 2003). Given risperidone has been used clinically as an antipsychotic for a number of years, the dosing, safety, and side effects are well understood. These factors could allow a relatively quick approval process for the use of risperidone as a treatment of MS if clinical trials were successful. This combination of factors suggests further investigation into the therapeutic potential of risperidone as a treatment for MS is merited.

\subsubsection{Future directions}

To identify the specific mechanisms by which risperidone alters macrophage activation, the effect of neurotransmitter receptor antagonism by risperidone should be explore; as this is the most likely explanation for risperidone's effects. These studies could be conducted though the use of receptor specific agonists (ie. to competitively alter risperidone's antagonistic effects) or though receptor specific antagonists to identify specific receptor activity. Alternately identification of alterations in intracellular signalling, such as cAMP, which could be initiated or inhibited when particular receptors are antagonised may provide insight into risperidone's mechanism of action.

It was not determined if the alteration in macrophage phenotype induced by risperidone is stable in the absence of risperidone. This effect could be easy clarified by identifying if phenotypic changes persist after risperidone is washed out from the cultures. Additionally co-culture of macrophages with stimulated $\mathrm{T}$ cells could provide insight into whether risperidone-treated macrophages had a regulatory effect on $\mathrm{T}$ cells. To ascertain if in vitro risperidone-treated macrophages had regulatory effects in vivo, cultured risperidone-treated macrophages could be injected into EAE immunised mice to identify if they alter disease 
expression. Any further research should also explore whether similar effects occur in human-derived macrophage cultures.

Assuming risperidone's effects on in vitro microglial and macrophages cultures occurs in vivo, it is likely the altered phenotype of microglia and macrophages would lead to a reduction in chronic CNS inflammation. Therefore, further studies should explore potential phenotypic changes of macrophages and microglia that occur following in vivo risperidone administration, and whether these changes result in altered cellular composition of CNS lesions or inhibition of myelin destruction. These studies could be conducted using histology and flow cytometry based techniques.

Although oral administration via the drinking water allows for relatively stable concentrations of risperidone, the short half life in rodents means that there are still likely to be peaks and troughs of drug concentration as dictated by the amount of water consumed at a particular time of day. It is therefore conceivable that risperidone would be even more effective a reducing disease severity were consistent concentrations maintained. To investigate this risperidone could be administered via controlled release implants or osmotic minipumps (Aravagiri et al., 1998; Kapur et al., 2003). As risperidone is only one of a number of atypical antipsychotics that have similar receptor antagonistic activity, the immunomodulatory potential of other compounds within this class of drugs could also be explored.

\subsection{MIS416}

\subsubsection{Summary}

The current study has demonstrated that EAE is an appropriate model for which to elucidate the immunomodulatory effects of MIS416. MIS416 was demonstrated to be effective in the treatment of EAE when administered immediately before immunisation, during disease onset or when a low dose was administered weekly. Additionally this study provided evidence for the involvement of IFN- $\gamma$ in MIS416 disease modification. 


\subsubsection{Clinical implications}

Anecdotal reports from pre-clinical, compassionate use of MIS416 indicates that MIS416 has minimal side effects and provides benefits to the progressive MS patients (personal communication; Gill Webster, Innate Immunotherapeutics). A phase 2A trial is currently underway to evaluate the safety and tolerability of MIS416. If this trial proves to be effective further in-depth trials will be conducted.

For drugs to be approved for clinical use by regulatory agencies, such as the FDA, a large body of evidence needs to be provided indicating the safety and effectiveness of the new therapy (Lipsky et al., 2001). Animal studies have a fundamental role in the approval process. Additionally, having a comprehensive understanding of the drug's mechanism of action facilitates the process of gaining regulatory approval. Identification of mechanisms of action usually occurs through intensive investigations in animal models, and the current study has demonstrated that EAE is an appropriate model for exploring how MIS416 alters an MS-relevant disease process. The findings from this study provide a foundation for future research and assist in the process of developing MIS416 as clinical therapeutic for MS.

\subsubsection{Future directions}

It was demonstrated that IFN- $\gamma$ is a necessary factor for effective disease modification by MIS416. What specific role IFN- $\gamma$ has, the cells that are producing it, and the location from which it is acting, remain to be determined. Additionally, whether or not IFN- $\gamma$ is necessary only during disease induction or if it also has a regulatory role in the chronic phase of disease would be interesting to investigate. As MIS416 can induce the production of type I interferons in vitro, the potential of MIS416 to induce production of these cytokines and the effect they have on EAE should be explored (Girvan et al., 2010).

Changes in cellularity induced by MIS416 were not investigated in the current study. Insight into the mechanisms by with which MIS416 suppresses disease may be gained through analysing changes in specific immune cell populations. Populations of particular interest would include regulatory subsets such a Tregs, MDSC or certain macrophages 
populations. Additionally NK cells, which can be activated by NOD2 and TLR9 ligands, could also have a regulatory role (Fu et al., 2011; Xu et al., 2011). The increase in IFN- $\gamma$ after MIS416 treatment may indicate, or result in, altered ratios of Th1/Th17 populations; therefore identifying changes in these populations may also contribute to understanding how MIS416 alters EAE expression.

It is also unknown if the suppression of disease in MIS416-treated mice is due to alterations in peripheral immune responses or due to processes occurring within the CNS. Analysis of CNS lesions and oligodendrocyte pathology could indicate whether MIS416 alters lesion development (eg. via alterations in immune events occurring in the CNS) or if MIS416 has a direct protective effect on oligodendrocytes (eg. by inhibiting cell death). Additionally, direct effects of MIS416 on the process of demyelination and remyelination could further be studied using other animal models, such the cuprizone-induced demyelination model (Matsushima et al., 2001).

As it appeared that the effects of lower doses (i.e. $100 \mu \mathrm{g}$ ) of MIS416 began to diminish within 7 days of administration, it would be interesting to explore if more frequent dosing, for example once every 4 days further enhanced the efficacy of MIS416. Currently MIS416 is administered by intravenous injection; however, the ideal administration route would be oral as it is much less invasive for patients who would be regularly taking MIS416. It therefore would be interesting to explore whether oral administration could also induce similar regulatory effects in EAE. 
References 
Aapro M, Finek J (2011). Oral vinorelbine in metastatic breast cancer: A review of current clinical trial results. Cancer Treatment Reviews In Press, Corrected Proof.

Abal M, Andreu JM, Barasoain I (2003). Taxanes: Microtubule and Centrosome Targets, and Cell Cycle Dependent Mechanisms of Action. Current Cancer Drug Targets 3(3): 193-203.

Abbas AK, Kenneth MM, Sher A (1996). Functional Diversity of Helper T Lymphocytes. Nature 383: 787-793.

Abraham SN, St. John AL (2010). Mast cell-orchestrated immunity to pathogens. Nature Reviews Immunology 10(6): 440-452.

Aharoni R, Eilam R, Stock A, Vainshtein A, Shezen E, Gal H, et al. (2010). Glatiramer acetate reduces Th-17 inflammation and induces regulatory T-cells in the CNS of mice with relapsing-remitting or chronic EAE. Journal of Neuroimmunology 225(1-2): 100-111.

Aharoni R, Teitelbaum D, Sela M, Arnon R (1997). Copolymer 1 induces T cells of the T helper type 2 that crossreact with myelin basic protein and suppress experimental autoimmune encephalomyelitis. Proceedings of the National Academy of Sciences of the United States of America 94(20): 10821-10826.

Ajami B, Bennett JL, Krieger C, McNagny KM, Rossi FMV (2011). Infiltrating monocytes trigger EAE progression, but do not contribute to the resident microglia pool. Nature Neuroscience 14(9): 1142-1149.

Akira S, Hemmi H (2003). Recognition of pathogen-associated molecular patterns by TLR family. Immunology Letters 85(2): 85-95.

Akira S, Uematsu S, Takeuchi O (2006). Pathogen Recognition and Innate Immunity. Cell 124(4): 783-801.

Albina JE, Abate JA, Henry WL (1991). Nitric oxide production is required for murine resident peritoneal macrophages to suppress mitogen-stimulated $\mathrm{T}$ cell proliferation. Role of IFN-gamma in the induction of the nitric oxide-synthesizing pathway. The Journal of Immunology 147(1): 144-148.

Alegre M-L, Frauwirth KA, Thompson CB (2001). T-cell regulation by CD28 and CTLA4. Nature Reviews Immunology 1(3): 220-228.

Altmann K-H (2001). Microtubule-stabilizing agents: a growing class of important anticancer drugs. Current Opinion in Chemical Biology 5(4): 424-431.

Amor S, Groome N, Linington C, Morris MM, Dornmair K, Gardinier MV, et al. (1994). Identification of Epitopes of Myelin Oligodendrocyte Glycoprotien for the Induction of 
Experimental Allergic Encephalomyelitis in SJL and Biozzi AB/H Mice. The Journal of Immunology 153(10): 4349.

Anderson CF, Gerber JS, Mosser DM (2002a). Modulating macrophage function with IgG immune complexes. Journal of Endotoxin Research 8(6): 477-481.

Anderson CF, Mosser DM (2002b). A novel phenotype for an activated macrophage: The type 2 activated macrophage. Journal of Leukocyte Biology 72(1): 101-106.

Angiotech-Pharmaceuticals (2002). Micellar paclitaxel does not appear to be effective in patients with multiple sclerosis. Inpharma Weekly 1328(11).

Aravagiri M, Yuwiler A, Marder SR (1998). Distribution after repeated oral administration of different dose levels of risperidone and 9-hydroxy-risperidone in the brain and other tissues of rat. Psychopharmacology 139(4): 356-363.

Arnold D, Campagnolo D, Panitch H, Bar-Or A, Dunn J, Freedman M, et al. (2008). Glatiramer acetate after mitoxantrone induction improves MRI markers of lesion volume and permanent tissue injury in MS. Journal of Neurology 255(10): 1473-1478.

Arnon R, Aharoni R (2004). Mechanism of action of glatiramer acetate in multiple sclerosis and its potential for the development of new applications. PNAS 101(SUPPL. 2): 14593-14598.

Artaloytia JF, Arango C, Lahti A, Sanz J, Pascual A, Cubero P, et al. (2006). Negative signs and symptoms secondary to antipsychotics: A double-blind, randomized trial of a single dose of placebo, haloperidol, and risperidone in healthy volunteers. American Journal of Psychiatry 163(3): 488-493.

Ascherio A, Munger K (2011). Epstein-Barr Virus Infection and Multiple Sclerosis: A Review. Journal of Neuroimmune Pharmacology 5(3): 271-277.

Ascherio A, Munger KL, Simon KC (2010). Vitamin D and multiple sclerosis. The Lancet Neurology 9(6): 599-612.

Axtell RC, de Jong BA, Boniface K, van der Voort LF, Bhat R, De Sarno P, et al. (2010). T helper type 1 and 17 cells determine efficacy of interferon-[beta] in multiple sclerosis and experimental encephalomyelitis. Nat Med 16(4): 406-412.

Bach J (2002). The Effect of Infections on Susceptibility to Autoimmune and Allergic Diseases. New England Journal of Medicine 347(12): 911-920.

Baker D, Gerritsen W, Rundle J, Amor S (2011). Critical appraisal of animal models of multiple sclerosis. Multiple Sclerosis 17(6): 647-657. 
Banwell B, Krupp L, Kennedy J, Tellier R, Tenembaum S, Ness J, et al. (2007). Clinical features and viral serologies in children with multiple sclerosis: a multinational observational study. The Lancet Neurology 6(9): 773-781.

Bar-Or A, Oliveira EML, Anderson DE, Hafler DA (1999). Molecular pathogenesis of multiple sclerosis. Journal of Neuroimmunology 100(1-2): 252-259.

Barnett MH, Prineas JW (2004). Relapsing and Remitting Multiple Sclerosis: Pathology of the Newly Forming Lesion. Annals of Neurology 55(4): 458-468.

Bartzokis G, Lu PH, Nuechterlein KH, Gitlin M, Doi C, Edwards N, et al. (2007). Differential effects of typical and atypical antipsychotics on brain myelination in schizophrenia. Schizophrenia Research 93(1-3): 13-22.

Bartzokis G, Lu PH, Stewart SB, Oluwadara B, Lucas AJ, Pantages J, et al. (2009). In vivo evidence of differential impact of typical and atypical antipsychotics on intracortical myelin in adults with schizophrenia. Schizophrenia Research 113(2-3): 322-331.

Basta-kaim A, Budziszewska G, Jagla G, Nowak W, Kubera M, Lason W (2006). Inhibitory effect of antipsychotic drugs on the Con A and LPS- induced proliferative activity of mouse splenocytes: A possble mechanism of action. Journal of Physiology and Pharmacology 57(2): 247-264.

Batista FD, Harwood NE (2009). The who, how and where of antigen presentation to B cells. Nature Review Immunology 9(1): 15-27.

Behi ME, Dubucquoi S, Lefranc D, ZÃ@phir Hln, Seze JrD, Vermersch P, et al. (2005). New insights into cell responses involved in experimental autoimmune encephalomyelitis and multiple sclerosis. Immunology Letters 96(1): 11-26.

Belotti D, Vergani V, Drudis T, Borsotti P, Pitelli MR, Viale G, et al. (1996). The microtubule-affecting drug paclitaxel has antiangiogenic activity. Clinical Cancer Research 2(11): 1843-1849.

Belotto KCR, Raposo NdRB, Ferreira AS, Gattaz WF (2010). Relative bioavailability of two oral formulations of risperidone $2 \mathrm{mg}$ : A single-dose, randomized-sequence, openlabel, two-period crossover comparison in healthy Brazilian volunteers. Clinical Therapeutics 32(12): 2106-2115.

Berenbaum MC (1985). The expected effect of a combination of agents: the general solution. Journal of Theoretical Biology 114(3): 413-431.

Berghmans N, Nuyts A, Uyttenhove C, Van Snick J, Opdenakker G, Heremans H (2011). Interferon- $\gamma$ orchestrates the number and function of Th17 cells in experimental autoimmune encephalomyelitis. Journal of Interferon and Cytokine Research 31(7): 575587. 
Bernard CCA, Johns TG, A. S, Ichikawa M, Ewing C, Liu J, et al. (1997). Myelin Oligodendrocyte Glycoprotein: A novel Candidate Autoantigen in Multiple Sclerosis. Journal of Molecular Medicine 75: 77-88.

Berridge MV, Herst PM, Tan AS, El-Gewely MR (2005). Tetrazolium dyes as tools in cell biology: New insights into their cellular reduction. Biotechnology Annual Review Volume 11: $127-152$.

Berridge MV, Tan AS (1993). Characterization of the Cellular Reduction of 3-(4,5dimethylthiazol-2-yl)-2,5-diphenyltetrazolium bromide (MTT): Subcellular Localization, Substrate Dependence, and Involvement of Mitochondrial Electron Transport in MTT Reduction. Archives of Biochemistry and Biophysics 303(2): 474-482.

Bettelli E, Pagany M, Weiner HL, Linington C, Sobel RA, Kuchroo VK (2003). Myelin oligodendrocyte glycoprotein-specific $\mathrm{T}$ cell receptor transgenic mice develop spontaneous autoimmune optic neuritis. Journal of Experimental Medicine 197(9): 1073-1081.

Bian Q, Kato T, Monji A, Hashioka S, Mizoguchi Y, Horikawa H, et al. (2008). The effect of atypical antipsychotics, perospirone, ziprasidone and quetiapine on microglial activation induced by interferon-[gamma]. Progress in Neuro-Psychopharmacology and Biological Psychiatry 32(1): 42-48.

Billiau A, Heremans H, Vandekerckhove F, Dijkmans R, Sobis H, Meulepas E, et al. (1988). Enhancement of experimental allergic encephalomyelitis in mice by antibodies against IFN-gamma. The Journal of Immunology 140(5): 1506-1510.

Bonneville M, O'Brien RL, Born WK (2010). $\gamma \alpha$ T cell effector functions: a blend of innate programming and acquired plasticity. Nat Rev Immunol 10(7): 467-478.

Borge B, Iversen V, Reed R (2006). Changes in plasma protein extravasation in rat skin during inflammatory challenges evaluated by microdialysis. Heart and Circulatory Physiology 290(5): H2108-H2115.

Boster A, Bartoszek MP, O'Connell C, Pitt D, Racke M (2011a). Efficacy, safety, and costeffectiveness of glatiramer acetate in the treatment of relapsingâ€"remitting multiple sclerosis. Therapeutic Advantages in Neurological Disorders 4(5): 319-332.

Boster A, Bartoszek MP, Oâ€TMConnell C, Pitt D, Racke M (2011b). Efficacy, safety, and cost-effectiveness of glatiramer acetate in the treatment of relapsingâ€"remitting multiple sclerosis. Therapeutic Advantages in Neurological Disorders 4(5): 319-332.

Bowden CR, Voina SJ, Woestenborghs R, De Coster R, Heykants J (1992). Stimulation by risperidone of rat prolactin secretion in vivo and in cultured pituitary cells in vitro. The journal of Pharmacology 262(2): 699-706. 
Brahn E, Tang C, Banquerigo ML (1994). Regression of collagen-induced arthritis with taxol, a microtubule stabilizer. Arthritis and Rheumatism 37(6): 839-845.

Braun MC, Kelsall BL (2001). Regulation of interleukin-12 production byG-proteincoupled receptors. Microbes and Infection 3(2): 99-107.

Brinkmann V (2009). FTY720 (fingolimod) in Multiple Sclerosis: therapeutic effects in the immune and the central nervous system. British Journal of Pharmacology 158(5): 1173-1182.

Bronstad A, Berg A, Reed RK (2004). Effects of the taxanes paclitaxel and docetaxel on edema formation and interstitial fluid pressure. Heart and Circulatory Physiology 287(2): H963-H968.

Bronstein-Sitton N, Cohen-Daniel L, Vaknin I, Ezernitchi AV, Leshem B, Halabi A, et al. (2003). Sustained exposure to bacterial antigen induces interferon-y-dependent $\mathrm{T}$ cell receptor down-regulation and impaired T cell function. Nature Immunology 4(10): 957964.

Brown DL, Little JE, Chaly N (1985). Effects of taxol on microtubule organization in mouse splenic lymphocytes and on response to mitogenic stimulation. European Journal of Cell Biology VOL. 37: 130-139.

Brown SW, Meyers RT, Brennan KM, Rumble JM, Narasimhachari N, Perozzi EF, et al. (2003). Catecholamines in a macrophage cell line. Journal of Neuroimmunology 135(1-2): 47-55.

Brunden KR, Yao Y, Potuzak JS, Ferrer NI, Ballatore C, James MJ, et al. (2011). The characterization of microtubule-stabilizing drugs as possible therapeutic agents for Alzheimer's disease and related tauopathies. Pharmacological Research 63(4): 341-351.

Bryceson YT, March ME, Ljunggren H-G, Long EO (2006). Activation, coactivation, and costimulation of resting human natural killer cells. Immunological Reviews 214(1): 73-91.

Byrd-Leifer CA, Block EF, Takeda K, Akira S, Ding A (2001). The role of MyD88 and TLR4 in the LPS-mimetic activity of Taxol. European Journal of Immunology 31(8): 2448-2457.

Cabre P, Signate A, Olindo S, Merle H, Caparros-Lefebvre D, Bera O, et al. (2005). Role of return migration in the emergence of multiple sclerosis in the French West Indies. Brain 128(12): 2899-2910.

Calle Y, Burns S, Thrasher AJ, Jones GE (2006). The leukocyte podosome. European Journal of Cell Biology 85(3-4): 151-157. 
Campbell DJ, Koch MA (2011). Phenotypical and functional specialization of FOXP3+ regulatory T cells. Nature Reviews Immunology 11(2): 119-130.

Cao L, Sun D, Cruz T, Moscarello MA, Ludwin SK, Whitaker JN (2000). Inhibition of experimental allergic encephalomyelitis in the Lewis rat by paclitaxel. Journal of Neuroimmunology 108(1-2): 103-111.

Carlson T, Kroenke M, Rao P, Lane TE, Segal B (2008). The Th17-ELR+ CXC chemokine pathway is essential for the development of central nervous system autoimmune disease. Journal of Experimental Medicine 205(4): 811-823.

Carrithers MD, Visintin I, Viret C, Janeway Jr CA (2002). Role of genetic background in P selectin-dependent immune surveillance of the central nervous system. Journal of Neuroimmunology 129(1-2): 51-57.

Carson MJ, Doose JM, Melchior B, Schmid CD, Ploix CC (2006). CNS immune privilege: Hiding in plain sight. Immunological Reviews 213(1): 48-65.

Carty M, Bowie AG (2011). Evaluating the role of Toll-like receptors in diseases of the central nervous system. Biochemical Pharmacology 81(7): 825-837.

Casadevall A, Pirofski L-a (2004). New Concepts in Antibody-Mediated Immunity. Infection and Immunity 72(11): 6191-6196.

Chan A, Andreae P, Northcote P, Miller J (2011). Peloruside A inhibits microtubule dynamics in a breast cancer cell line MCF7. Investigational New Drugs 29(4): 615-626.

Chapagain ML, Sumibcay L, Gurjav U, Kaufusi PH, Kast RE, Nerurkar VR (2008). Serotonin receptor $2 \mathrm{~A}$ blocker (risperidone) has no effect on human polyomavirus JC infection of primary human fetal glial cells. Journal of Neurovirology 14(5): 448-454.

Chapagain ML, Verma S, Mercier F, Yanagihara R, Nerurkar VR (2007). Polyomavirus JC infects human brain microvascular endothelial cells independent of serotonin receptor $2 \mathrm{~A}$. Virology 364(1): 55-63.

Chaturvedi V, Collison LW, Guy CS, Workman CJ, Vignali DAA (2011). Cutting Edge: Human Regulatory T Cells Require IL-35 To Mediate Suppression and Infectious Tolerance. The Journal of Immunology 186(12): 6661-6666.

Chaudhuri A (2005). Lessions for clinical trails from natalizumab in multiple sclerosis British Medical Journal 332: 416-419.

Chemel B, Roth B, Armbruster B, Watts V, Nichols D (2006). WAY-100635 is a potent dopamine D\&lt;sub\&gt;4\&lt;/sub\&gt; receptor agonist. Psychopharmacology 188(2): 244251. 
Chou T-C, Talalay P (1984). Quantitative analysis of dose-effect relationships: the combined effects of multiple drugs or enzyme inhibitors. Advances in Enzyme Regulation 22(0): 27-55.

Choy JC (2010). Granzymes and perforin in solid organ transplant rejection. Cell Death Differerentation 17(4): 567-576.

Chuang LT, LotzovÃ $;$ E, Heath J, Cook KR, Munkarah A, Morris M, et al. (1994). Alteration of lymphocyte microtubule assembly, cytotoxicity, and activation by the anticancer drug taxol. Cancer Research 54(5): 1286-1291.

Ciofani M, Zuniga-Pflucker JC (2010). Determining $\gamma \delta$ versus $\alpha \beta$ T cell development. Nat Rev Immunol 10(9): 657-663.

Clark EA, Hills PM, Davidson BS, Wender PA, Mooberry SL (2006). Laulimalide and Synthetic Laulimalide Analogues Are Synergistic with Paclitaxel and 2-Methoxyestradiol. Molecular Pharmaceutics 3(4): 457-467.

Cobham M, Donovan D (2009). Ixabepilone: a new treatment option for the management of taxane-resistant metastatic breast cancer. Cancer Management and Research 1: 69-77.

Cohen JA, Barkhof F, Comi G, Hartung H-P, Khatri BO, Montalban X, et al. (2010). Oral Fingolimod or Intramuscular Interferon for Relapsing Multiple Sclerosis. The New England Journal of Medicine 362(5): 402-415.

Collison LW, Workman CJ, Kuo TT, Boyd K, Wang Y, Vignali KM, et al. (2007). The inhibitory cytokine IL-35 contributes to regulatory T-cell function. Nature 450(7169): 566569.

Colmenarez HJ, Escaned J, Fernández C, Lobo L, Cano S, del Angel JG, et al. (2010). Efficacy and Safety of Drug-Eluting Stents in Chronic Total Coronary Occlusion Recanalization: A Systematic Review and Meta-Analysis. Journal of the American College of Cardiology 55(17): 1854-1866.

Condamine T, Gabrilovich DI (2011). Molecular mechanisms regulating myeloid-derived suppressor cell differentiation and function. Trends in Immunology 32(1): 19-25.

Confavreux C, Vukusic S (2006). Accumulation of irreversible disability in multiple sclerosis: From epidemiology to treatment. Clinical Neurology and Neurosurgery 108(3): 327-332.

Consortium R (2011). Genetic risk and a primary role for cell-mediated immune mechanisms in multiple sclerosis. Nature 476(7359): 214-219.

Conway D, Cohen JA (2008). Combination therapy in multiple sclerosis. The Lancet Neurology 9(3): 299-308. 
Corbeil P, Rodrigue J, Simoneau M, Cohen H, Pourcher E (2011). Influence of risperidone on balance control in young healthy individuals. Psychopharmacology 11: 1-11.

Correale J, Farez M (2007). Association between parasite infection and immune responses in multiple sclerosis. Annals of Neurology 61(2): 97-108.

Correale J, Farez M, Razzitte G (2008). Helminth infections associated with multiple sclerosis induce regulatory B cells. Annals of Neurology 64(2): 187-199.

Correale J, Farez MF (2011). The impact of environmental infections (parasites) on MS activity. Multiple Sclerosis 17(10): 1162-1169.

Cosentino M, Fietta AM, Ferrari M, Rasini E, Bombelli R, Carcano E, et al. (2007). Human CD4+CD25+ regulatory $\mathrm{T}$ cells selectively express tyrosine hydroxylase and contain endogenous catecholamines subserving an autocrine/paracrine inhibitory functional loop. Blood 109(2): 632-642.

Cosentino M, Zaffaroni M, Marino F, Bombelli R, Ferrari M, Rasini E, et al. (2002). Catecholamine production and tyrosine hydroxylase expression in peripheral blood mononuclear cells from multiple sclerosis patients: effect of cell stimulation and possible relevance for activation-induced apoptosis. Journal of Neuroimmunology 133(1-2): 233240.

Costantino C, Baecher-Allan C, Hafler D (2008). Multiple Sclerosis and Regulatory T Cells. Journal of Clinical Immunology 28(6): 697-706.

Crown J, O'Leary M, Ooi W-S (2004). Docetaxel and Paclitaxel in the Treatment of Breast Cancer: A Review of Clinical Experience. The Oncologist 9(suppl 2): 24-32.

Crume KP Modification of the Disease Progression in Mice with Experimental Autoimmune Encephalomyelitis Using Anti-Mitotic Compounds. PhD, Victoria University of Wellington 2007.

Crume KP, Miller JH, La Flamme AC (2007). Peloruside A, an Antimitotic Agent, Specifically Decreases Tumor Necrosis Factor-\{alpha\} Production by LipopolysaccharideStimulated Murine Macrophages. Experimental Biology and Medicine 232(5): 607-613.

Crume KP, O'Sullivan D, Miller JH, Northcote PT, La Flamme AC (2009). Delaying the onset of experimental autoimmune encephalomyelitis with the microtubule-stabilizing compounds, paclitaxel and Peloruside A. Journal of Leukocyte Biology 86: jlb.0908541.

Cua DJ, Sherlock J, Chen Y, Murphy CA, Joyce B, Seymour B, et al. (2003). Interleukin23 rather than interleukin-12 is the critical cytokine for autoimmune inflammation of the brain. Nature 421(6924): 744-748. 
Dalton DK, Pitts-Meek S, Keshav S, Figari IS, Bradley A, Stewart TA (1993). Multiple defects of immune cell function in mice with disrupted interferon-gamma genes. Science 259(5102): 1739-1742.

Dardalhon V, Awasthi A, Kwon H, Galileos G, Gao W, Sobel RA, et al. (2008). IL-4 inhibits TGF-b-induced Foxp3+ T cells and, together with TGF-b, generates IL-9+ IL-10+ Foxp3- effector T cells. Nature Immunology 9(12): 1347-1355.

DeAngelis T, Lublin F (2008). Multiple sclerosis: New treatment trials and emerging therapeutic targets. Current Opinion in Neurology 21(3): 261-271.

Delgado M, Martinez C, Pozo D, Calvo JR, Leceta J, Ganea D, et al. (1999). Vasoactive Intestinal Peptide (VIP) and Pituitary Adenylate Cyclase-Activation Polypeptide (PACAP) Protect Mice from Lethal Endotoxemia Through the Inhibition of TNF- $\mathrm{I} \pm$ and IL-6. The Journal of Immunology 162(2): 1200-1205.

Dello Russo C, Boullerne AI, Gavrilyuk V, Feinstein DL (2004). Inhibition of microglial inflammatory responses by norepinephrine: Effects on nitric oxide and interleukin-I $\hat{~}^{2}$ production. Journal of Neuroinflammation 1.

Denardo SJ, Kukis DL, Kroger LA, O'Donnell RT, Lamborn KR, Miers LA, et al. (1997). Synergy of Taxol and radioimmunotherapy with yttrium-90-labeled chimeric L6 antibody: Efficacy and toxicity in breast cancer xenografts. Proceedings of the National Academy of Sciences of the United States of America 94(8): 4000-4004.

Deng X, Sriram S (2005). Role of microglia in multiple sclerosis. Current Neurology and Neuroscience Reports 5(3): 239-244.

Dhib-Jalbut S, Marks S (2010). Interferon-b mechanisms of action in multiple sclerosis. Neurology 74(1 Supplement 1): S17-S24.

Dimitriadou V, Pang X, Theoharides TC (2000). Hydroxyzine inhibits experimental allergic encephalomyelitis (EAE) and associated brain mast cell activation. International Journal of Immunopharmacology 22(9): 673-684.

Dimitrijevic M, PilipoviÄc I, StanojeviÄc S, MitiÄc K, Radojevic K, PeÅjï̈c V, et al. (2009). Chronic propranolol treatment affects expression of adrenoceptors on peritoneal macrophages and their ability to produce hydrogen peroxide and nitric oxide. Journal of Neuroimmunology 211(1-2): 56-65.

Dimopoulou I, Bamias A, Lyberopoulos P, Dimopoulos MA (2006). Pulmonary toxicity from novel antineoplastic agents. Annals of Oncology 17(3): 372-379.

Disanto G, Berlanga A, Handel A, Para A, Burrell A, Fries A, et al. (2011). Heterogeneity in Multiple Sclerosis: Scratching the Surface of a Complex Disease. Autoimmune Disease 2011: 12 . 
Donald H G (2005). Infectious causes of multiple sclerosis. The Lancet Neurology 4(3): 195-202.

Drzyzga L, Obuchowicz E, Marcinowska A, Herman ZS (2006). Cytokines in schizophrenia and the effects of antipsychotic drugs. Brain, Behaviour, and Immunity 20(6): 532-545.

Duong TT, Finkelman FD, Singh B, Strejan GH (1994a). Effect of Anti-Interferon- $\gamma$ Monoclonal Antibody Treatment on the Development of Experimental Allergic Encephalomyelitis in Resistant Mouse Strains. Journal of Neuroimmunology 53: 101-107.

Duong TT, Finkelman FD, Singh B, Strejan GH (1994b). Effect of anti-interferon-y monoclonal antibody treatment on the development of experimental allergic encephalomyelitis in resistant mouse strains. Journal of Neuroimmunology 53(1): 101-107.

Dyment DA, Herrera BM, Cader MZ, Willer CJ, Lincoln MR, Sadovnick AD, et al. (2005). Complex interactions among MHC haplotypes in multiple sclerosis: susceptibility and resistance. Human Molecular Genetics 14(14): 2019-2026.

Dyment DA, Sadnovich AD, Ebers GC (1997). Genetics of Multiple Sclerosis. Human Molecular Genetics 6(10): 1693-1698.

Edwards JF, Slauson DO (1983). Complete Freund's adjuvant-induced pneumonia in swine: A model of interstitial lung disease. Journal of Comparative Pathology 93(3): 353-361.

Edwards JP, Zhang X, Frauwirth KA, Mosser DM (2006). Biochemical and functional characterization of three activated macrophage populations. Journal of Leukocyte Biology 80(6): 1298-1307.

Ehrlich A, Booher S, Becerra Y, Borris DL, Figg WD, Turner ML, et al. (2004). Micellar paclitaxel improves severe psoriasis in a prospective phase II pilot study. Journal of the American Academy of Dermatology 50(4): 533-540.

Elenkov IJ, Webster E, Papanicolaou DA, Fleisher TA, Chrousos GP, Wilder RL (1998). Histamine Potently Suppresses Human IL-12 and Stimulates IL-10 Production via H2 Receptors. The Journal of Immunology 161(5): 2586-2593.

Elphick GF, Querbes W, Jordan JA, Gee GV, Eash S, Manley K, et al. (2004). The Human Polyomavirus, JCV, Uses Serotonin Receptors to Infect Cells. Science 306(5700): 13801383.

Engelhardt B (2010). T cell migration into the central nervous system during health and disease: Different molecular keys allow access to different central nervous system compartments. Clinical and Experimental Neuroimmunology 1(2): 79-93. 
Engelhardt B, Laschinger M, Schulz M, Samulowitz U, Vestweber D, Hoch G (1998a). The development of experimental autoimmune encephalomyelitis in the mouse requires alpha4-integrin but not alpha4beta7-integrin. The Journal of Clinical Investigation 102(12): 2096-2105.

Engelhardt B, Martin-Simonet M-t, Rott LS, Butcher EC, Michie SA (1998b). Adhesion molecule phenotype of T lymphocytes in inflamed CNS. Journal of Neuroimmunology 84(1): 92-104.

Erickson HP, O'Brien ET (1992). Microtubule Dynamic Instability and GTP Hydrolysis Vol. 21, pp 145-166.

Espejo C, Penkowa M, Saez-Torres I, Xaus J, Celada A, Montalban X, et al. (2001). Treatment with Anti-interferon-y Monoclonal Antibodies Modifies Experimental Autoimmune Encephalomyelitis in Interferon-y Receptor Knockout Mice. Experimental Neurology 172(2): 460-468.

Esposito F, Radaelli M, Martinelli V, Sormani MP, Martinelli Boneschi F, Moiola L, et al. (2010). Comparative study of mitoxantrone efficacy profile in patients with relapsing"remitting and secondary progressive multiple sclerosis. Multiple Sclerosis 16(12): 14901499.

Esquifino AI, Cano P, Zapata A, Cardinali DP (2006). Experimental allergic encephalomyelitis in pituitary-grafted Lewis rats. Journal of Neuroinflammation 3.

Eyerich S, Eyerich K, Pennino D, Carbone T, Nasorri F, Pallotta S, et al. (2009). Th22 cells represent a distinct human $\mathrm{T}$ cell subset involved in epidermal immunity and remodeling. The Journal of Clinical Investigation 119(12): 3573-3585.

Facchinetti F, Del Giudice E, Furegato S, Passarotto M, Arcidiacono D, Leon A (2004). Dopamine inhibits responses of astroglia-enriched cultures to lipopolysaccharide via a beta-adrenoreceptor-mediated mechanism. Journal of Neuroimmunology 150(1-2): 29-36.

Fathman CG, Lineberry NB (2007). Molecular mechanisms of CD4+ T-cell anergy. Nature Reviews Immunology 7(8): 599-609.

Fellner S, Bauer B, Miller DS, Schaffrik M, FankhÃơnel M, SpruÃŸ T, et al. (2002). Transport of paclitaxel (taxol) across the blood-brain barrier in vitro and in vivo. Journal of Clinical Investigation 110(9): 1309-1318.

Feng WG, Wang YB, Zhang JS, Wang XY, Li CL, Chang ZL (2002). cAMP elevators inhibit LPS-induced IL-12 p40 expression by interfering with phosphorylation of p38 MAPK in Murine Peritoneal Macrophages. Cell Research 12(5-6): 331-337. 
Ferber IA, Brocke S, Taylor-Edwards C, Ridgway W, Dinisco C, Steinman L, et al. (1996a). Mice with a disrupted IFN- $\gamma$ gene are susceptible to the induction of experimental autoimmune encephalomyelitis (EAE). Journal of Immunology 156(1): 5-7.

Ferber IA, Brocke S, Taylor-Edwards C, Ridgway W, Dinisco C, Steinman L, et al. (1996b). Mice with a disrupted IFN-gamma gene are susceptible to the induction of experimental autoimmune encephalomyelitis (EAE). The Journal of Immunology 156(1): $5-7$.

Fernandez M, Montalban X, Comabella M (2010). Orchestrating innate immune responses in multiple sclerosis: Molecular players. Journal of Neuroimmunology 225(1-2): 5-12.

Fisher GA, Lum BL, Hausdorff J, Sikic BI (1996). Pharmacological considerations in the modulation of multidrug resistance. European Journal of Cancer 32(6): 1082-1088.

Fitzgerald P, Dinan TG (2008). Prolactin and dopamine: What is the connection? A Review Article. Psychopharmacology 22(2 suppl): 12-19.

Fitzpatrick FA, Wheeler R (2003). The immunopharmacology of paclitaxel (Taxol®), docetaxel (Taxotere $\left.{ }^{\circledR}\right)$, and related agents. International Immunopharmacology 3(13-14): 1699-1714.

Fleming JO, Isaak A, Lee JE, Luzzio CC, Carrithers MD, Cook TD, et al. (2011). Probiotic helminth administration in relapsingâ€"remitting multiple sclerosis: a phase 1 study. Multiple Sclerosis 17(6): 743-754.

Fleming JOMD, Cook TDP (2006). Multiple sclerosis and the hygiene hypothesis. Neurology 67(11): 2085-2086.

Fletcher JM, Lalor SJ, Sweeney CM, Tubridy N, Mills KHG (2010). T cells in multiple sclerosis and experimental autoimmune encephalomyelitis. Clinical \& Experimental Immunology 162(1): 1-11.

Flierl MA, Rittirsch D, Nadeau BA, Chen AJ, Sarma JV, Zetoune FS, et al. (2007). Phagocyte-derived catecholamines enhance acute inflammatory injury. Nature 449(7163): 721-725.

Floris S, Ruuls SR, Wierinckx A, van der Pol SMA, Dopp E, van der Meide PH, et al. (2002). Interferon-b directly influences monocyte infiltration into the central nervous system. Journal of Neuroimmunology 127(1-2): 69-79.

Flugel A, Berkowicz T, Ritter T, Labeur M, Jenne DE, Li Z, et al. (2001). Migratory activity and functional changes of green fluorescent effector cells before and during experimental autoimmune encephalomyelitis. Immunity 14(5): 547-560. 
Focosi D, Fazzi R, Montanaro D, Emdin M, Petrini M (2007). Progressive multifocal leukoencephalopathy in a haploidentical stem cell transplant recipient: A clinical, neuroradiological and virological response after treatment with risperidone. Antiviral Research 74(2): 156-158.

Fojo T, Menefee M (2007). Mechanisms of multidrug resistance: the potential role of microtubule-stabilizing agents. Annals of Oncology 18(suppl 5): v3-v8.

Francisco LM, Sage PT, Sharpe AH (2010). The PD-1 pathway in tolerance and autoimmunity. Immunological Reviews 236(1): 219-242.

Franco R, Pacheco R, Lluis C, Ahern GP, O'Connell PJ (2007). The emergence of neurotransmitters as immune modulators. Trends in Immunology 28(9): 400-407.

Freire-Garabal M, Nunez MJ, Balboa J, Garcia-Vallejo LA, Argibay S, Rodrigo E, et al. (2003). Administration of the 5-hydroxytryptamine(1A) receptor antagonist WAY100635 suppresses acute experimental allergic encephalomyelitis in Lewis rats. Neuroscience Letters 342(1-2): 33-36.

Friedl P, Weigelin B (2008). Interstitial leukocyte migration and immune function. Nature Immunology 9(9): 960-969.

Friese MA, Fugger L (2009). Pathogenic CD8+ T cells in multiple sclerosis. Annals of Neurology 66(2): 132-141.

Frohman EM, Racke MK, Raine CS (2006). Multiple Sclerosis - The Plaque and Its Pathogenesis. The New England Journal of Medicine 354(9): 942-955.

Fu Q, Maniar A, Quevedo Diaz M, Chapoval AI, Medvedev AE (2011). Activation of cytokine-producing and antitumor activities of natural killer cells and macrophages by engagement of Toll-like and NOD-like receptors. Innate Immunity 17(4): 375-387.

Fujimori K, Yokoyama A, Kurita Y, Uno K, Saijo N (1998). Paclitaxel-induced cellmediated hypersensitivity pneumonitis. Diagnosis using leukocyte migration test, bronchoalveolar lavage and transbronchial lung biopsy. Oncology 55: 340-344.

Furlan R, Brambilla E, Ruffini F, Poliani PL, Bergami A, Marconi PC, et al. (2001). Intrathecal Delivery of IFN- $\hat{I}^{3}$ Protects C57BL/6 Mice from Chronic-Progressive Experimental Autoimmune Encephalomyelitis by Increasing Apoptosis of Central Nervous System-Infiltrating Lymphocytes. The Journal of Immunology 167(3): 1821-1829.

Furtado GuC, Marcondes MCG, Latkowski J-A, Tsai J, Wensky A, Lafaille JJ (2008). Swift Entry of Myelin-Specific T Lymphocytes into the Central Nervous System in Spontaneous Autoimmune Encephalomyelitis. Journal of Immunology 181(7): 4648-4655. 
Gabrilovich DI, Nagaraj S (2009). Myeloid-derived suppressor cells as regulators of the immune system. Nature Reviews Immunology 9(3): 162-174.

Gaitanos TN, Buey RM, Diaz JF, Northcote PT, Teesdale-Spittle P, Andreu JM, et al. (2004). Peloruside A Does Not Bind to the Taxoid Site on $\hat{\mathrm{I}}^{2}$-Tubulin and Retains Its Activity in Multidrug-Resistant Cell Lines Vol. 64, pp 5063-5067.

Galligan CL, Pennell LM, Murooka TT, Baig E, Majchrzak-Kita B, Rahbar R, et al. (2010). Interferon- $b$ is a key regulator of proinflammatory events in experimental autoimmune encephalomyelitis Vol. 16, pp 1458-1473.

Gapud EJ, Bai R, Ghosh AK, Hamel E (2004). Laulimalide and Paclitaxel: A Comparison of Their Effects on Tubulin Assembly and Their Synergistic Action When Present Simultaneously. Molecular Pharmacology 66(1): 113-121.

Gay FW, Drye TJ, Dick GW, Esiri MM (1997). The application of multifactorial cluster analysis in the staging of plaques in early multiple sclerosis. Identification and characterization of the primary demyelinating lesion. Brain 120(8): 1461-1483.

Gelderblom H, Verweij J, Nooter K, Sparreboom A (2001). Cremophor EL: the drawbacks and advantages of vehicle selection for drug formulation. European Journal of Cancer 37(13): 1590-1598.

Gelderblom H, Verweij J, van Zomeren DM, Buijs D, Ouwens L, Nooter K, et al. (2002). Influence of Cremophor EL on the Bioavailability of Intraperitoneal Paclitaxel. Clinical Cancer Research 8(4): 1237-1241.

Genain CP, Cannella B, Hauser SL, Raine CS (1999). Identification of autoantibodies associated with myelin damage in multiple sclerosis. Nature Medicine 5(2): 170.

George C E (2008). Environmental factors and multiple sclerosis. The Lancet Neurology 7(3): 268-277.

Gerber JS, Mosser DM (2001). Reversing lipopolysaccharide toxicity by ligating the macrophage $\mathrm{Fc}^{3}$ receptors. Journal of Immunology 166(11): 6861-6868.

Ghosh AK, Hirasawa N, Ohtsu H, Watanabe T, Ohuchi K (2002). Defective Angiogenesis in the Inflammatory Granulation Tissue in Histidine Decarboxylase-deficient Mice but not in Mast Cell-deficient Mice. Journal of Experimental Medicine 195(8): 973-982.

Giovannoni G, Munschauer FE, Deisenhammer F (2002). Neutralising antibodies to interferon beta during the treatment of multiple sclerosis. Journal of Neurology, Neurosurgery and Psychiatry 73(5): 465-469.

Girvan RC, Knight DA, O'loughlin CJ, Hayman CM, Hermans IF, Webster GA (2010). MIS416, a non-toxic microparticle adjuvant derived from Propionibacterium acnes 
comprising immunostimulatory muramyl dipeptide and bacterial DNA promotes crosspriming and Th1 immunity. Vaccine 29(3): 545-557.

Girvin AM, Dal Canto MC, Miller SD (2002). CD40/CD40L Interaction is Essential for the Induction of EAE in the Absence of CD28-Mediated Co-stimulation. Journal of Autoimmunity 18(2): 83-94.

Gold R (2008). Combination therapies in multiple sclerosis. Journal of Neurology 255(0): 51-60.

Gold R, Hartung H-P, Toyka KV (2000). Animal Models for Autoimmune Demyelinating Disorders of the Nervous System Molecular Medicine Today 6: 88-91.

Gold R, Linington C, Lassmann H (2006). Understanding Pathogenesis and Therapy of Multiple Sclerosis via Animal Models: 70 years of Merits and Culprits in Experimental Autoimmune Encephalomyelitis Research Brain 129: 1953-1971.

Goverman J (2009). Autoimmune T cell responses in the central nervous system. Nature Reviews Immunology 9(6): 393-407.

Gran B, Chu N, Zhang G-X, Yu S, Li Y, Chen X-H, et al. (2004). Early administration of IL-12 suppresses EAE through induction of interferon-y. Journal of Neuroimmunology 156(1-2): 123-131.

Greer J, M., Sobel RA, Stette A, Southwood S, Lees MB, Kuchroo V (1996). Immunogenic and Encephalitogenic Epitope Clusters of Myelin Proteolipid Protien. The Journal of Immunology 156: 371-379.

Gregg C, Shikar V, Larsen P, Mak G, Chojnacki A, Yong VW, et al. (2007). White Matter Plasticity and Enhanced Remyelination in the Maternal CNS. The Journal of Neuroscience 27(8): 1812-1823.

Grewal IS, Flavell RA (1998). CD40 AND CD154 IN CELL-MEDIATED IMMUNITY. Annual review of Immunology 16(1): 111-135.

Grewal IS, Foellmer HG, Grewal KD, Xu J, Hardardottir F, Baron JL, et al. (1996). Requirement for CD40 Ligand in Costimulation Induction, T Cell Activation, and Experimental Allergic Encephalomyelitis Vol. 273, pp 1864-1867.

Guillin O, Abi-Dargham A, Laruelle M (2007). Neurobiology of Dopamine in Schizophrenia. In: (ed)^(eds). International Review of Neurobiology, edn, Vol. Volume 78: Academic Press. p^pp 1-39.

Gutzmer R, Diestel C, Mommert S, KÃ Ither B, Stark H, Wittmann M, et al. (2005). Histamine H4 Receptor Stimulation Suppresses IL-12p70 Production and Mediates 
Chemotaxis in Human Monocyte-Derived Dendritic Cells. The Journal of Immunology 174(9): 5224-5232.

Haas J, Hug A, Viehöver A, Fritzsching B, Falk CS, Filser A, et al. (2005). Reduced suppressive effect of $\mathrm{CD} 4+\mathrm{CD} 25$ high regulatory $\mathrm{T}$ cells on the $\mathrm{T}$ cell immune response against myelin oligodendrocyte glycoprotein in patients with multiple sclerosis. European Journal of Immunology 35(11): 3343-3352.

Hamel E, Day BW, Miller JH, Jung MK, Northcote PT, Ghosh AK, et al. (2006). Synergistic Effects of Peloruside A and Laulimalide with Taxoid Site Drugs, but Not with Each Other, on Tubulin Assembly. Molecular Pharmacology 70(5): 1555-1564.

Handel AE, Giovannoni G, Ebers GC, Ramagopalan SV (2010). Environmental factors and their timing in adult-onset multiple sclerosis.(Report). Nature Reviews Clinical Neurology 6(3): 156(111).

Handel AE, Williamson AJ, Disanto G, Dobson R, Giovannoni G, Ramagopalan SV (2011). Smoking and Multiple Sclerosis: An Updated Meta-Analysis. PLoS ONE 6(1): e16149.

Hartung H-P, Gonsette R, Konig N, Kwiecinski H, Guseo A, Morrissey SP, et al. (2002). Mitoxantrone in progressive multiple sclerosis: a placebo-controlled, double-blind, randomised, multicentre trial. The Lancet 360(9350): 2018-2025.

Harty JT, Tvinnereim AR, White DW (2000). CD8+ T Cell Effector Mechanisms in Resistance to Infection. Annual review of Immunology 18(1): 275-308.

Haskó G, Szabó C, Németh ZH, Deitch EA (2002). Dopamine suppresses IL-12 p40 production by lipopolysaccharide-stimulated macrophages via a [beta]-adrenoceptormediated mechanism. Journal of Neuroimmunology 122(1-2): 34-39.

Hawiger D, Flavell RA (2009). Regulatory T cells that become autoaggressive. Nature Immunology 10(9): 938-939.

Hayashi T, Gray C, Chan M, Tawatao R, Ronacher L, McGargill M, et al. (2009). Prevention of autoimmune disease by induction of tolerance to Toll-like receptor 7. PNAS 106(8): 2764-2769.

Heesen C, Schulz H, Schmidt M, Gold S, Tessmer W, Schulz KH (2002). Endocrine and cytokine responses to acute psychological stress in multiple sclerosis. Brain, Behavior, and Immunity 16(3): 282-287.

Henderson APD, Barnett MH, Parratt JDE, Prineas JW (2009). Multiple sclerosis: Distribution of inflammatory cells in newly forming lesions. Annals of Neurology 66(6): 739-753. 
Herz J, Zipp F, Siffrin V (2009). Neurodegeneration in autoimmune CNS inflammation. Experimental Neurology 225(1): 9-17.

Hirokawa N (1998). Kinesin and Dynein Superfamily Proteins and the Mechanism of Organelle Transport. Science 279(5350): 519-526.

Ho HH, Antoniv TT, Ji J-D, Ivashkiv LB (2008). Lipopolysaccharide-Induced Expression of Matrix Metalloproteinases in Human Monocytes Is Suppressed by IFN-y via Superinduction of ATF-3 and Suppression of AP-1. The Journal of Immunology 181(7): 5089-5097.

Hofstetter HH, Forsthuber TG (2010). Kinetics of IL-17- and interferon-[gamma]producing PLPp-specific CD4 T cells in EAE induced by coinjection of PLPp/IFA with pertussis toxin in SJL mice. Neuroscience Letters 476(3): 150-155.

Hofstetter HH, Ibrahim SM, Koczan D, Kruse N, Weishaupt A, Toyka KV, et al. (2005). Therapeutic efficacy of IL-17 neutralization in murine experimental autoimmune encephalomyelitis. Cellular Immunology 237(2): 123-130.

Hogan SP, Rosenberg HF, Moqbel R, Phipps S, Foster PS, Lacy P, et al. (2008). Eosinophils: Biological Properties and Role in Health and Disease. Clinical \& Experimental Allergy 38(5): 709-750.

Hong J, Li N, Zhang X, Zheng B, Zhang JZ (2005). Induction of CD4+CD25+ regulatory T cells by copolymer-I through activation of transcription factor Foxp3. PNAS 102(18): 6449-6454.

Hong S, Van Kaer L (1999). Immune Privilege. Journal of Experimental Medicine 190(9): 1197-1200.

Horacek J, Bubenikova-Valesova V, Kopecek M, Palenicek T, Dockery C, Mohr P, et al. (2006). Mechanism of Action of Atypical Antipsychotic Drugs and the Neurobiology of Schizophrenia. CNS Drugs 20(5): 389.

Hu X, Ivashkiv LB (2009). Cross-regulation of Signaling Pathways by Interferon-y: Implications for Immune Responses and Autoimmune Diseases. Immunity 31(4): 539-550.

Huang GS, Lopez-Barcons L, Freeze BS, Smith AB, Goldberg GL, Horwitz SB, et al. (2006). Potentiation of Taxol Efficacy by Discodermolide in Ovarian Carcinoma Xenograft-Bearing Mice. Clinical Cancer Research 12(1): 298-304.

Hutchinson M (2007). Natalizumab: A new treatment for relapsing remitting multiple sclerosis Theraputics and Clinical Risk Management 2(2): 259-268. 
Inohara N, Koseki T, Lin J, del Peso L, Lucas PC, Chen FF, et al. (2000). An Induced Proximity Model for NF-1 ${ }^{\circ} \mathrm{B}$ Activation in the Nod1/RICK and RIP Signaling Pathways. Journal of Biological Chemistry 275(36): 27823-27831.

Itoh Y, Sendo T, Hirakawa T, Goromaru T, Takasaki S, Yahata H, et al. (2004). Role of Sensory Nerve Peptides Rather than Mast Cell Histamine in Paclitaxel Hypersensitivity. American Journal of Respiratory and Critical Care Medicine 169(1): 113-119.

Ivanov II, McKenzie BS, Zhou L, Tadokoro CE, Lepelley A, Lafaille JJ, et al. (2006). The Orphan Nuclear Receptor ROR $\hat{I}^{3} \mathrm{t}$ Directs the Differentiation Program of Proinflammatory IL-17+ T Helper Cells. Cell 126(6): 1121-1133.

Jack C, Ruffini F, Bar-Or A, Antel JP (2005). Microglia and multiple sclerosis. Journal of Neuroscience Research 81(3): 363-373.

Jadidi-Niaragh F, Mirshafiey A (2010). Histamine and histamine receptors in pathogenesis and treatment of multiple sclerosis. Neuropharmacology 59(3): 180-189.

Jain N, Nguyen H, Chambers C, Kang J (2010). Dual function of CTLA-4 in regulatory T cells and conventional T cells to prevent multiorgan autoimmunity. PNAS 107(4): 15241528.

Janeway CA, Medzhitov R (2002). Innate Immune Recognition. Annual review of Immunology 20(1): 197-216.

Jasny E, Eisenblãătter M, MÃătz-Rensing K, Tenner-Racz K, Tenbusch M, Schrod A, et al. (2008). IL-12-Impaired and IL-12-Secreting Dendritic Cells Produce IL-23 upon CD154 Restimulation. The Journal of Immunology 180(10): 6629-6639.

Jee Y, Liu R, Bai X-F, Campagnolo DI, Shi F-D, Vollmer TL (2006). Do Th2 cells mediate the effects of glatiramer acetate in experimental autoimmune encephalomyelitis? International Immunology 18(4): 537-544.

Jee Y, Piao WH, Liu R, Bai XF, Rhodes S, Rodebaugh R, et al. (2007). CD4+CD25+ regulatory $\mathrm{T}$ cells contribute to the therapeutic effects of glatiramer acetate in experimental autoimmune encephalomyelitis. Clinical Immunology 125(1): 34-42.

Jenkins SJ, Ruckerl D, Cook PC, Jones LH, Finkelman FD, van Rooijen N, et al. (2011). Local Macrophage Proliferation, Rather than Recruitment from the Blood, Is a Signature of TH2 Inflammation. Science 332(6035): 1284-1288.

Jenner RG, Townsend MJ, Jackson I, Sun K, Bouwman RD, Young RA, et al. (2009). The transcription factors T-bet and GATA-3 control alternative pathways of T-cell differentiation through a shared set of target genes. PNAS 106(42): 17876-17881. 
John J, Ismail M, Riley C, Askham J, Morgan R, Melcher A, et al. (2010). Differential effects of Paclitaxel on dendritic cell function. BMC Immunology 11(1): 14.

Johnson T, Jirik F, Fournier S (2010). Exploring the roles of CD8\&lt;sup\&gt;+\&lt;/sup\&gt; $\mathrm{T}$ lymphocytes in the pathogenesis of autoimmune demyelination. Seminars in Immunopathology 32(2): 197-209.

Jonnakuty C, Gragnoli C (2008). What do we know about serotonin? Journal of Cellular Physiology 217(2): 301-306.

Jordan MA, Kamath K (2007). How do microtubule-targeted drugs work? An overview. Current Cancer Drug Targets 7(8): 730-742.

Jordan MA, Wilson L (2004). Microtubules as a target for anticancer drugs. Nature Reviews Cancer 4(4): 253-265.

Juedes AE, HjelmstrÃ̃Im P, Bergman CM, Neild AL, Ruddle NH (2000). Kinetics and Cellular Origin of Cytokines in the Central Nervous System: Insight into Mechanisms of Myelin Oligodendrocyte Glycoprotein-Induced Experimental Autoimmune Encephalomyelitis. Journal of Immunology 164(1): 419-426.

Kala M, Miravalle A, Vollmer T (2011). Recent insights into the mechanism of action of glatiramer acetate. Journal of Neuroimmunology 235(1-2): 9-17.

Kamath K, Jordan MA (2003). Suppression of Microtubule Dynamics by Epothilone B Is Associated with Mitotic Arrest. Cancer Research 63(18): 6026-6031.

Kappos L, Antel J, Comi G, Montalban X, O'Connor P, Polman CH, et al. (2006). Oral Fingolimod (FTY720) for Relapsing Multiple Sclerosis. New England Journal of Medicine 355(11): 1124-1140.

Kappos L, Radue E-W, O'Connor P, Polman C, Hohlfeld R, Calabresi P, et al. (2010). A Placebo-Controlled Trial of Oral Fingolimod in Relapsing Multiple Sclerosis Vol. 362, pp 387-401.

Kapur S, Langlois X, Vinken P, Megens AAHP, De Coster R, Andrews JS (2002). The Differential Effects of Atypical Antipsychotics on Prolactin Elevation Are Explained by Their Differential Blood-Brain Disposition: A Pharmacological Analysis in Rats. The Journal of Pharmacology and Experimental Theraputics 302(3): 1129-1134.

Kapur S, Remington G (2001). Dopamine D2 receptors and their role in atypical antipsychotic action: still necessary and may even be sufficient. Biological Psychiatry 50(11): 873-883.

Kapur S, Vanderspek SC, Brownlee BA, Nobrega JN (2003). Antipsychotic dosing in preclinical models is often unrepresentative of the clinical condition: A suggested solution 
based on in vivo occupancy. Journal of Pharmacology and Experimental Therapeutics 305(2): 625-631.

Karandikar NJ, Vanderlugt CL, Eagar T, Tan L, Bluestone JA, Miller SD (1998). TissueSpecific Up-Regulation of B7-1 Expression and Function During the Course of Murine Relapsing Experimental Autoimmune Encephalomyelitis. The Journal of Immunology 161(1): 192-199.

Karasuyama H, Mukai K, Obata K, Tsujimura Y, Wada T (2011). Nonredundant Roles of Basophils in Immunity. Annual Review of Immunology 29(1): 45-69.

Karl T, Duffy L, O'Brien E, Matsumoto I, Dedova I (2006). Behavioural effects of chronic haloperidol and risperidone treatment in rats. Behavioural Brain Research 171(2): 286-294.

Karpus WJ, Ransohoff RM (1998). Cutting Edge Commentary: Chemokine Regulation of Experimental Autoimmune Encephalomyelitis: Temporal and Spatial Expression Patterns Govern Disease Pathogenesis. The Journal of Immunology 161(6): 2667-2671.

Karussis D, Teitelbaum D, Sicsic C, Brenner T (2010). Long-term treatment of multiple sclerosis with glatiramer acetate: Natural history of the subtypes of anti-glatiramer acetate antibodies and their correlation with clinical efficacy. Journal of Neuroimmunology 220(1â€"'2): 125-130.

Kast RE, Focosi D, Petrini M, Altschuler EL (2007). Treatment schedules for 5-HT2A blocking in progressive multifocal leukoencephalopathy using risperidone or ziprasidone. Bone Marrow Transplant 39(12): 811-812.

Kataoka H, Sugahara K, Shimano K, Teshima K, Koyama M, Fukunari A, et al. (2005). FTY720, sphingosine 1-phosphate receptor modulator, ameliorates experimental autoimmune encephalomyelitis by inhibition of T cell infiltration. Cellular \& molecular immunology. 2(6): 439-448.

Kato T, Mizoguchi Y, Monji A, Horikawa H, Suzuki SO, Seki Y, et al. (2008). Inhibitory effects of aripiprazole on interferon- $\gamma$-induced microglial activation via intracellular $\mathrm{Ca} 2+$ regulation in vitro. Journal of Neurochemistry 106(2): 815-825.

Kato T, Monji A, Hashioka S, Kanba S (2007). Risperidone significantly inhibits interferon-[gamma]-induced microglial activation in vitro. Schizophrenia Research 92(1-3): 108-115.

Kawai T, Akira S (2010). The role of pattern-recognition receptors in innate immunity: update on Toll-like receptors. Nature Immunology 11(5): 373-384.

Keating P, O'Sullivan D, Tierney JB, Kenwright D, Miromoeini S, Mawasse L, et al. (2009). Protection from EAE by IL-4Ra-/- macrophages depends upon T regulatory cell involvement. Immunology and Cell Biology. 
Keegan BM, Noseworthy JH (2002). Multiple Sclerosis Annual Review of Medicine 53(1): 285-302.

Kemper EM, Verheij M, Boogerd W, Beijnen JH, van Tellingen O (2004). Improved penetration of docetaxel into the brain by co-administration of inhibitors of P-glycoprotein. European Journal of Cancer 40(8): 1269-1274.

Khrapunovich-Baine M, Menon V, Yang C-PH, Northcote PT, Miller JH, Angeletti RH, et al. (2011). Hallmarks of Molecular Action of Microtubule Stabilizing Agents: EFFECTS OF EPOTHILONE B, IXABEPILONE, PELORUSIDE A, AND LAULIMALIDE ON MICROTUBULE CONFORMATION. Journal of Biological Chemistry 286(13): 1176511778.

Kim HJ, Ifergan I, Antel JP, Seguin R, Duddy M, Lapierre Y, et al. (2004). Type 2 Monocyte and Microglia Differentiation Mediated by Glatiramer Acetate Therapy in Patients with Multiple Sclerosis. The Journal of Immunology 172(11): 7144-7153.

Kivisäkk P, Imitola J, Rasmussen S, Elyaman W, Zhu B, Ransohoff RM, et al. (2009). Localizing central nervous system immune surveillance: Meningeal antigen-presenting cells activate T cells during experimental autoimmune encephalomyelitis. Annals of Neurology 65(4): 457-469.

Kleinschek MA, Owyang AM, Joyce-Shaikh B, Langrish CL, Chen Y, Gorman DM, et al. (2007). IL-25 regulates Th17 function in autoimmune inflammation. Journal of Experimental Medicine 204(1): 161-170.

Knable MB, Heinz A, Raedler T, Weinberger DR (1997). Extrapyramidal side effects with risperidone and haloperidol at comparable D2 receptor occupancy levels. Psychiatry Research: Neuroimaging 75(2): 91-101.

Komiyama Y, Nakae S, Matsuki T, Nambu A, Ishigame H, Kakuta S, et al. (2006a). IL-17 Plays an Important Role in the Development of Experimental Autoimmune Encephalomyelitis. The Journal of Immunology 177(1): 566-573.

Komiyama Y, Nakae S, Matsuki T, Nambu A, Ishigame H, Kakuta S, et al. (2006b). IL-17 plays an important role in the development of experimental autoimmune encephalomyelitis. Journal of Immunology 177(1): 566-573.

Korn T, Bettelli E, Oukka M, Kuchroo VK (2009). IL-17 and Th17 Cells. Annual Review of Immunology 27: 485-517.

Korn T, Reddy J, Gao W, Bettelli E, Awasthi A, Petersen TR, et al. (2007). Myelinspecific regulatory $\mathrm{T}$ cells accumulate in the CNS but fail to control autoimmune inflammation. Nature Medicine 13(4): 423-431. 
Kovarik JM, Hartmann S, Bartlett M, Riviere G-J, Neddermann D, Wang Y, et al. (2007). Oral-intravenous crossover study of fingolimod pharmacokinetics, lymphocyte responses and cardiac effects. Biopharmaceutics \& Drug Disposition 28(2): 97-104.

Krammer PH (2000). CD95's deadly mission in the immune system. Nature 407(6805): 789-795.

Kreisel D, Nava RG, Li W, Zinselmeyer BH, Wang B, Lai J, et al. (2010). In vivo twophoton imaging reveals monocyte-dependent neutrophil extravasation during pulmonary inflammation. PNAS 107(42): 18073-18078.

Krenger W, Falzarano G, Delmonte J, Jr., Snyder KM, Byon JC, Ferrara JL (1996). Interferon-gamma suppresses T-cell proliferation to mitogen via the nitric oxide pathway during experimental acute graft-versus-host disease. Blood 88(3): 1113-1121.

Kuerten S, Rottlaender A, Rodi M, Velasco Jr VB, Schroeter M, Kaiser C, et al. (2010). The clinical course of EAE is reflected by the dynamics of the neuroantigen-specific $\mathrm{T}$ cell compartment in the blood. Clinical Immunology 137(3): 422-432.

Kwidzinski E, Bunse Jr, Aktas O, Richter D, Mutlu L, Zipp F, et al. (2005). Indolamine 2,3-dioxygenase is expressed in the CNS and down-regulates autoimmune inflammation. FASEB 6.

Larochelle C, Alvarez JI, Prat A (2011). How do immune cells overcome the blood-brain barrier in multiple sclerosis? FEBS Letters(0).

Latchman YE, Liang SC, Wu Y, Chernova T, Sobel RA, Klemm M, et al. (2004). PD-L1deficient mice show that PD-L1 on T cells, antigen-presenting cells, and host tissues negatively regulates T cells. PNAS 101(29): 10691-10696.

Lautenschlager F, Paschke S, Schinkinger S, Bruel A, Beil M, Guck J (2009). The regulatory role of cell mechanics for migration of differentiating myeloid cells.

Proceedings of the National Academy of Sciences of the United States of America 106(37): 15696-15701.

Leary S, Thompson A (2005). Primary Progressive Multiple Sclerosis : Current and Future Treatment Options. CNS Drugs 19(5): 369-376.

Lee C, Choi J, Chun J (2010). Neurological S1P signaling as an emerging mechanism of action of oral FTY720 (Fingolimod) in multiple sclerosis. Archives of Pharmacal Research 33(10): 1567-1574.

Leucht S, Pitschel-Walz G, Abraham D, Kissling W (1999). Efficacy and extrapyramidal side-effects of the new antipsychotics olanzapine, quetiapine, risperidone, and sertindole compared to conventional antipsychotics and placebo. A meta-analysis of randomized controlled trials. Schizophrenia Research 35(1): 51-68. 
Ley S, Weigert A, Brüne B (2010). Neuromediators in inflammation--a macrophage/nerve connection. Immunobiology 215(9-10): 674-684.

Li B, Greene MI (2008). Special regulatory T-cell review: FOXP3 biochemistry in regulatory $\mathrm{T}$ cells - how diverse signals regulate suppression. Immunology 123(1): 17-19.

Li Z, Woo CJ, Iglesias-Ussel MD, Ronai D, Scharff MD (2004). The generation of antibody diversity through somatic hypermutation and class switch recombination. Genes and Development 18(1): 1-11.

Libbey JE, McCoy LL, Fujinami RS, Alireza M (2007). Molecular Mimicry in Multiple Sclerosis. International Review of Neurobiology Volume 79: 127-147.

Limmroth V, Putzki N, Kachuck NJ (2011). The interferon beta therapies for treatment of relapsingâ€" "remitting multiple sclerosis: are they equally efficacious? A comparative review of open-label studies evaluating the efficacy, safety, or dosing of different interferon beta formulations alone or in combination. Threaputic Advances in Neurological Disorders 4(5): 281-296.

Linder S, Hufner K, Wintergerst U, Aefelbacher M (2000). Microtubule-dependent formation of podosomal adhesion structures in primary human macrophages. Journal of Cell Science 113(23): 4165-4176.

Lipsky MS, Sharp LK (2001). From idea to market: The drug approval process. Journal of the American Board of Family Practice 14(5): 362-367.

Loke Pn, Allison JP (2003). PD-L1 and PD-L2 are differentially regulated by Th1 and Th2 cells. PNAS 100(9): 5336-5341.

Loscher W, Potschka H (2005). Blood-brain barrier active efflux transporters: ATPbinding cassette gene family. NeuroRx 2(1): 86-98.

Louten J, Boniface K, de Waal Malefyt R (2009). Development and function of TH17 cells in health and disease. Journal of Allergy and Clinical Immunology 123(5): 1004-1011.

Lu H, Murtagh J, Schwartz EL (2006). The Microtubule Binding Drug Laulimalide Inhibits Vascular Endothelial Growth Factor-Induced Human Endothelial Cell Migration and Is Synergistic when Combined with Docetaxel (Taxotere). Molecular pharmacology 69(4): 1207-1215.

Lubin FD, Reingold SC (1996). Defining the Clinical Course of Multiple Sclerosis. Neurology 46: 907-911. 
Lucas RM, Ponsonby AL, Dear K, Valery P, Pender MP, Burrows JM, et al. (2011). Current and past Epstein-Barr virus infection in risk of initial CNS demyelination. Neurology 77(4): 371-379.

Lucchinetti C, Brück W, Parisi J, Scheithauer B, Rodriguez M, Lassmann H (2000). Heterogeneity of multiple sclerosis lesions: Implications for the pathogenesis of demyelination. Annals of Neurology 47(6): 707-717.

Lund FE, Randall TD (2010). Effector and regulatory B cells: modulators of CD4+ T cell immunity. Nature Review Immunology 10(4): 236-247.

Lutton JD, Winston R, Rodman TC (2004). Multiple Sclerosis: Etiological Mechanisms and Future Directions. Experimental Biology and Medicine 229(1): 12-20.

Lyons J-A, Ramsbottom MJ, Trotter J, L., Cross AH (2002). Identification of the Encephalitogenic Epitopes of CNS Proteolipid Protein in Balb/c Mice. Journal of Autoimmunity 19: 195-201.

Macsween KF, Crawford DH (2003). Epstein-Barr virus-recent advances. The Lancet Infectious Diseases 3(3): 131-140.

Maes M, Bocchio Chiavetto L, Bignotti S, Battisa Tura G-J, Pioli R, Boin F, et al. (2002). Increased serum interleukin- 8 and interleukin-10 in schizophrenic patients resistant to treatment with neuroleptics and the stimulatory effects of clozapine on serum leukemia inhibitory factor receptor. Schizophrenia Research 54(3): 281-291.

Magalhaes JG, Lee J, Geddes K, Rubino S, Philpott DJ, Girardin SE (2011). Essential role of Rip2 in the modulation of innate and adaptive immunity triggered by Nod1 and Nod2 ligands. European Journal of Immunology 41(5): 1445-1455.

Maginnis MS, Haley SA, Gee GV, Atwood WJ (2010). Role of N-linked glycosylation of the 5-HT2A receptor in JC virus infection. Journal of Virology 84(19): 9677-9684.

Mamdouh Z, Kreitzer GE, Muller WA (2008). Leukocyte transmigration requires kinesinmediated microtubule-dependent membrane trafficking from the lateral border recycling compartment. Journal of Experimental Medicine 205(4): 951-966.

Mamdouh Z, Mikhailov A, Muller WA (2009). Transcellular migration of leukocytes is mediated by the endothelial lateral border recycling compartment. Journal of Experimental Medicine 206(12): 2795-2808.

Manis JP, Tian M, Alt FW (2002). Mechanism and control of class-switch recombination. Trends in Immunology 23(1): 31-39. 
Mantovani A, Sica A, Sozzani S, Allavena P, Vecchi A, Locati M (2004). The chemokine system in diverse forms of macrophage activation and polarization. Trends in immunology 25(12): 677-686.

Marriott JJMD, Miyasaki JMMDMF, Gronseth GMDF, O'Connor PWMDM (2010). Evidence Report: The efficacy and safety of mitoxantrone (Novantrone) in the treatment of multiple sclerosis: Report of the Therapeutics and Technology Assessment Subcommittee of the American Academy of Neurology (CME). Neurology 74(18): 1463-1470.

Marshall JS (2004). Mast-cell responses to pathogens. Nature Reviews Immunology 4(10): 787-799.

Marta M, Andersson Å, Isaksson M, Kämpe O, Lobell A (2008). Unexpected regulatory roles of TLR4 and TLR9 in experimental autoimmune encephalomyelitis. European Journal of Immunology 38(2): 565-575.

Marta M, Meier UC, Lobell A (2009). Regulation of autoimmune encephalomyelitis by toll-like receptors. Autoimmunity Reviews 8(6): 506-509.

Martello LA, McDaid HM, Regl DL, Yang C-PH, Meng D, Pettus TRR, et al. (2000). Taxol and Discodermolide Represent a Synergistic Drug Combination in Human Carcinoma Cell Lines. Clinical Cancer Research 6(5): 1978-1987.

Martiney JA, Rajan AJ, Charles PC, Cerami A, Ulrich PC, Macphail S, et al. (1998). Prevention and Treatment of Experimental Autoimmune Encephalomyelitis by CNI-1493, a Macrophage-Deactivating Agent. The Journal of Immunology 160(11): 5588-5595.

Matheu MP, Cahalan MD, Parker I (2011). Immunoimaging: Studying Immune System Dynamics Using Two-Photon Microscopy. Cold Spring Harbor Protocols 2011(2): pdb.top99.

Mathur RK, Awasthi A, Wadhone P, Ramanamurthy B, Saha B (2004). Reciprocal CD40 signals through p38MAPK and ERK-1/2 induce counteracting immune responses. Nature Medicine 10(5): 540-544.

Matsushima GK, Morell P (2001). The Neurotoxicant, Cuprizone, as a Model to Study Demyelination and Remyelination in the Central Nervous System. Brain Pathology 11(1): 107-116.

McColl SR, Staykova MA, Wozniak A, Fordham S, Bruce J, Willenborg DO (1998). Treatment with Anti-Granulocyte Antibodies Inhibits the Effector Phase of Experimental Autoimmune Encephalomyelitis. The Journal of Immunology 161(11): 6421-6426.

McFarland HF, Martin R (2007). Multiple sclerosis: a complicated picture of autoimmunity. Nature Immunology 8(9): 913-919. 
McGeachy MJ, Stephens LA, Anderton SM (2005). Natural Recovery and Protection from Autoimmune Encephalomyelitis: Contribution of CD4+CD25+ Regulatory Cells within the Central Nervous System. The Journal of Immunology 175(5): 3025-3032.

McHeyzer-Williams LJ, McHeyzer-Williams MG (2005). Antigen-specific memory B cell development. Annual Review of Immunology 23: 487-513.

McNamara R, Jandacek R, Rider T, Tso P (2011). Chronic risperidone normalizes elevated pro-inflammatory cytokine and C-reactive protein production in omega-3 fatty acid deficient rats. European Journal of Pharmacology 652(1): 152-156.

McNeill A, Spittle E, Bäckström BT (2007). Partial Depletion of CD69low-expressing Natural Regulatory T Cells with the Anti-CD25 Monoclonal Antibody PC61.

Scandinavian Journal of Immunology 65(1): 63-69.

McQualter JL, Bernard CCA (2007). Multiple sclerosis: a battle between destruction and repair. Journal of Neurochemistry 100(2): 295-306.

Medzhitov R, Janeway Jr CA (1997). Innate immunity: Impact on the adaptive immune response. Current Opinion in Immunology 9(1): 4-9.

Meltzer HY, Massey BW (2011). The role of serotonin receptors in the action of atypical antipsychotic drugs. Current Opinion in Pharmacology 11(1): 59-67.

Mendel I, Katz A, Kozak N, Ben-Nun A, Revel M (1998). Interleukin-6 functions in autoimmune encephalomyelitis: A study in gene-targeted mice. European Journal of Immunology 28(5): 1727-1737.

Merwe PAvd, Davis SJ (2003). MOLECULAR INTERACTIONS MEDIATING T CELL ANTIGEN RECOGNITION. Annual Review of Immunology 21(1): 659-684.

Meyer U, Schwarz MJ, Müller N (2011). Inflammatory processes in schizophrenia: A promising neuroimmunological target for the treatment of negative/cognitive symptoms and beyond. Pharmacology \& Therapeutics 132(1): 96-110.

Meylan E, Tschopp J, Karin M (2006). Intracellular pattern recognition receptors in the host response. Nature 442(7098): 39-44.

Miller SD, Vanderlugt CL, Lenschow DJ, Pope JG, Karandikar NJ, Dal Canto MC, et al. (1995). Blockade of CD28/B7-1 interaction prevents epitope spreading and clinical relapses of murine EAE. Immunity 3(6): 739-745.

Min B (2008). Basophils: what they 'can do' versus what they 'actually do'. Nature Immunology 9(12): 1333-1339. 
Minagar A, Alexander JS (2003). Blood-brain barrier disruption in multiple sclerosis. Multiple Sclerosis 9(6): 540-549.

Minguela A, Pastor S, Mi W, Richardson JA, Ward ES (2007). Feedback Regulation of Murine Autoimmunity via Dominant Anti-Inflammatory Effects of Interferon y. The Journal of Immunology 178(1): 134-144.

Miranda-Hernandez S, Gerlach N, Fletcher JM, Biros E, Mack M, KÃ Irner H, et al. (2011). Role for MyD88, TLR2 and TLR9 but Not TLR1, TLR4 or TLR6 in Experimental Autoimmune Encephalomyelitis. The Journal of Immunology 187(2): 791-804.

Missale C, Nash SR, Robinson SW, Jaber M, Caron MG (1998). Dopamine Receptors: From Structure to Function. Physiological Reviews 78(1): 189-225.

Moretta A, Marcenaro E, Parolini S, Ferlazzo G, Moretta L (2008). NK cells at the interface between innate and adaptive immunity. Cell Death and Differentiation 15(2): 226-233.

Morichika T, Takahashi HK, Iwagaki H, Yoshino T, Tamura R, Yokoyama M, et al. (2003). Histamine inhibits lipopolysaccharide-induced tumor necrosis factor- $\alpha$ production in an intercellular adhesion molecule-1- and B7.1-dependent manner. Journal of Pharmacology and Experimental Therapeutics 304(2): 624-633.

Mosmann T, Cherwinski H, Bond M, Giedlin M, Coffman R (1986). Two Types of Murine Helper T Cell Clone I. Definition According to Profiles of Lymphokine Activities and Secreted Proteins. The Journal of Immunology 136(7): 2348-2357.

Mosser DM (2003). The many faces of macrophage activation. Journal of Leukocyte Biology 73(2): 209-212.

Moulder SL, Holmes FA, Tolcher AW, Thall P, Broglio K, Valero V, et al. (2010). A randomized phase 2 trial comparing 3-hour versus 96-hour infusion schedules of paclitaxel for the treatment of metastatic breast cancer. Cancer 116(4): 814-821.

Mueller DL (2010). Mechanisms maintaining peripheral tolerance. Nature Immunology 11(1): 21-27.

Muller N, Hofschuster E, Ackenheil M, Eckstein R (1993). T-cells and psychopathology in schizophrenia: Relationship to the outcome of neuroleptic therapy. Acta Psychiatrica Scandinavica 87(1): 66-71.

Mumford CJ, Wood NW, Kellar-Wood H, Thorpe JW, Miller DH, Compston DAS (1994). The British Isles survey of multiple sclerosis in twins. Neurology 44(1): 11.

Munger KL, Zhang SM, O'Reilly E, HernÃ $; n$ MA, Olek MJ, Willett WC, et al. (2004). Vitamin D intake and incidence of multiple sclerosis. Neurology 62(1): 60-65. 
Munitz A, Levi-Schaffer F (2004). Eosinophils: 'new' roles for 'old' cells. Allergy 59(3): 268-275.

Murphy ÃiC, Lalor SJ, Lynch MA, Mills KHG (2010). Infiltration of Th1 and Th17 cells and activation of microglia in the CNS during the course of experimental autoimmune encephalomyelitis. Brain, Behavior, and Immunity 24(4): 641-651.

Nagata S, Ueda N, Yoshida Y, Matsuda H, Maehara Y (2010). Severe interstitial pneumonitis associated with the administration of taxanes. Journal of Infection and Chemotherapy 16(5): 340-344.

Naiker DV, Catts SV, Catts VS, Bedi KS, Bryan-Lluka LJ (2006). Dose determination of haloperidol, risperidone and olanzapine using an in vivo dopamine D2-receptor occupancy method in the rat. European Journal of Pharmacology 540(1-3): 87-90.

Nakano K, Higashi T, Hashimoto K, Takagi R, Tanaka Y, Matsushita S (2008). Antagonizing dopamine D1-like receptor inhibits Th17 cell differentiation: Preventive and therapeutic effects on experimental autoimmune encephalomyelitis. Biochemical and Biophysical Research Communications 373(2): 286-291.

Nakano K, Yamaoka K, Hanami K, Saito K, Sasaguri Y, Yanagihara N, et al. (2011). Dopamine Induces IL-6 Dependent IL-17 Production via D1-Like Receptor on CD4 Naive T Cells and D1-Like Receptor Antagonist SCH-23390 Inhibits Cartilage Destruction in a Human Rheumatoid Arthritis/SCID Mouse Chimera Model. The Journal of Immunology 186(6): 3745-3752.

Nakatsuji Y, Moriya M, Sakoda S (2007). Targeting interferons in multiple sclerosis. Drug Discovery Today: Therapeutic Strategies 4(1): 49-55.

Nathan C (2006). Neutrophils and immunity: challenges and opportunities. Nature Reviews Immunology 6(3): 173-182.

Neuhaus O, Kieseier BC, Hartung H-P (2006). Therapeutic role of mitoxantrone in multiple sclerosis. Pharmacology \& Therapeutics 109(1-2): 198-209.

Nielsen TR, Rostgaard K, Nielsen NM, Koch-Henriksen N, Haahr S, Sorensen PS, et al. (2007). Multiple Sclerosis After Infectious Mononucleosis. Archives of Neurology 64(1): $72-75$.

Nogales E (2001). STRUCTURAL INSIGHTS INTO MICROTUBULE FUNCTION. Annual Review of Biophysics and Biomolecular Structure 30(1): 397-420.

Noseworthy JH (1999). Progress in determining the causes and treatment of multiple sclerosis. Nature 399(Supplementary): A40-A47. 
Noyes K, Bajorska A, Chappel A, Schwid SR, Mehta LR, Weinstock-Guttman B, et al. (2011). Cost-effectiveness of disease-modifying therapy for multiple sclerosis. Neurology 77.

Nukuzuma S, Nakamichi K, Nukuzuma C, Takegami T (2009). Inhibitory effect of serotonin antagonists on JC virus propagation in a carrier culture of human neuroblastoma cells. Microbiology and Immunology 53(9): 496-501.

Nurieva RI, Liu X, Dong C (2009). Yin-Yang of costimulation: crucial controls of immune tolerance and function. Immunological Reviews 229(1): 88-100.

O'Brien K, Fitzgerald D, Rostami A, Gran B (2010). The TLR7 agonist, imiquimod, increases IFN-b production and reduces the severity of experimental autoimmune encephalomyelitis. Journal of Neuroimmunology 221(1-2): 107-111.

O'Connor RA, Leech MD, Suffner J, Hammerling GJ, Anderton SM (2010). Myelinreactive, TGF- $\hat{I}^{2}$-induced regulatory $\mathrm{T}$ cells can be programmed to develop Th1-like effector function but remain less proinflammatory than myelin-reactive Th1 effectors and can suppress pathogenic T cell clonal expansion in vivo. Journal of Immunology 185(12): 7235-7243.

O'Connor RA, Prendergast CT, Sabatos CA, Lau CWZ, Leech MD, Wraith DC, et al. (2008). Cutting Edge: Th1 Cells Facilitate the Entry of Th17 Cells to the Central Nervous System during Experimental Autoimmune Encephalomyelitis. The Journal of Immunology 181(6): $3750-3754$.

O'Connor TM, O'Connell J, O'Brien DI, Goode T, Bredin CP, Shanahan F (2004). The role of substance $\mathrm{P}$ in inflammatory disease. Journal of Cellular Physiology 201(2): 167-180.

O'Hara BA, Atwood WJ (2008). Interferon $\beta 1$-a and selective anti-5HT2a receptor antagonists inhibit infection of human glial cells by JC virus. Virus Research 132(1-2): 97103.

O'Shea JJ, Paul WE (2010). Mechanisms Underlying Lineage Commitment and Plasticity of Helper CD4+ T Cells. Science 327(5969): 1098-1102.

Okuda Y, Sakoda S, Fujimura H, Saeki Y, Kishimoto T, Yanagihara T (1999). IL-6 plays a crucial role in the induction phase of myelin oligodendrocyte glycoprotein 35-55 induced experimental autoimmune encephalomyelitis. Journal of Neuroimmunology 101(2): 188196.

Oliver SJ, Banquerigo ML, Brahn E (1994). Suppression of Collagen-Induced Arthritis Using an Angiogenesis Inhibitor, AGM-1470, and a Microtubule Stabilizer, Taxol. Cellular Immunology 157(1): 291-299. 
Ota K, Matsui M, Milford EL, Mackin GA, Weiner HL, Hafler DA (1990). T-cell recognition of an immuno-dominant myelin basic protein epitope in multiple sclerosis. Nature 346(6280): 183-187.

Pacheco R, Prado CE, Barrientos MJ, Bernales S (2009). Role of dopamine in the physiology of T-cells and dendritic cells. Journal of Neuroimmunology 216(1-2): 8-19.

Palmer E (2003). Negative selection - clearing out the bad apples from the T-cell repertoire. Nature Review Immunology 3(5): 383-391.

Parihar A, Eubank TD, Doseff AI (2010). Monocytes and macrophages regulate immunity through dynamic networks of survival and cell death. Journal of Innate Immunity 2(3): 204-215.

Parish CR, Glidden MH, Quah BJC, Warren HS (2009). Use of the intracellular fluorescent dye CFSE to monitor lymphocyte migration and proliferation. Current Protocols in Immunology(SUPPL. 84): 4.9.1-4.9.13.

Peachman KK, Rao M, Palmer DR, Zidanic M, Sun W, Alving CR, et al. (2004). Functional microtubules are required for antigen processing by macrophages and dendritic cells. Immunology Letters 95(1): 13-24.

Pender MP, Csurhes PA, Houghten RA, McCombe PA, Good MF (1996). A study of human T-cell lines generated from multiple sclerosis patients and controls by stimulation with peptides of myelin basic protein. Journal of Neuroimmunology 70(1): 65-74.

Perona-Wright G, Jenkins SJ, O'Connor RA, Zienkiewicz D, McSorley HJ, Maizels RM, et al. (2009). A Pivotal Role for CD40-Mediated IL-6 Production by Dendritic Cells during IL-17 Induction In Vivo. The Journal of Immunology 182(5): 2808-2815.

Pettinelli CB, Fritz R, Chou JC-H, Mcfarlin DE (1982). Encephalitogenic Activity of Guinea Pig Myelin Basic Protein in the SJL Mouse. The Journal of Immunology 129(3): 1209-1211.

Philippe C (2009). Environmental changes and epidemiology of multiple sclerosis in the French West Indies. Journal of the Neurological Sciences 286(1-2): 58-61.

Piascik MT, Perez DM (2001). $\alpha$-Adrenergic Receptors: New Insights and Directions. The journal of Pharmacology 298(2): 403-410.

Picca CC, Larkin J, Boesteanu A, Lerman MA, Rankin AL, Caton AJ (2006). Role of TCR specificity in CD4+CD25+ regulatory T-cell selection. Immunological Reviews 212(1): 7485 .

Pierrot-Deseilligny C, Souberbielle JC (2010). Is hypovitaminosis D one of the environmental risk factors for multiple sclerosis? Brain 133(7): 1869-1888. 
Piraino PS, Yednock TA, Freedman SB, Messersmith EK, Pleiss MA, Vandevert C, et al. (2002). Prolonged reversal of chronic experimental allergic encephalomyelitis using a small molecule inhibitor of a4 integrin. Journal of Neuroimmunology 131(1-2): 147-159.

Potvin S, Stip E, Sepehry AA, Gendron A, Bah R, Kouassi E (2008). Inflammatory Cytokine Alterations in Schizophrenia: A Systematic Quantitative Review. Biological Psychiatry 63(8): 801-808.

Prat M, Gribaudo G, Comoglio PM, Cavallo G, Landolfo S (1984). Monoclonal antibodies against murine gamma interferon. PNAS 81(14): 4515-4519.

Prinz M, Garbe F, Schmidt H, Mildner A, Gutcher I, Wolter K, et al. (2006). Innate immunity mediated by TLR9 modulates pathogenicity in an animal model of multiple sclerosis. The Journal of Clinical Investigation 116(2): 456-464.

Prinz M, Häusler KG, Kettenmann H, Hanisch U-K (2001). Beta-adrenergic receptor stimulation selectively inhibits IL-12p40 release in microglia. Brain Research 899(1-2): 264-270.

Pryor DE, O'Brate A, Bilcer G, Diaz JF, Wang Y, Wang Y, et al. (2002). The Microtubule Stabilizing Agent Laulimalide Does Not Bind in the Taxoid Site, Kills Cells Resistant to Paclitaxel and Epothilones, and May Not Require Its Epoxide Moiety for Activityâ€ Biochemistry 41(29): 9109-9115.

Pucci E, Giuliani G, Solari A, Simi S, Minozzi S, Di Pietrantonj C, et al. (2011). Natalizumab for relapsing remitting multiple sclerosis. Cochrane database of systematic reviews (Online)(10).

Pul R, Moharregh-Khiabani D, Å kuljec J, Skripuletz T, Garde N, VoÃ ̈̈ E, et al. (2011). Glatiramer Acetate Modulates TNF- $\alpha$ and IL-10 Secretion in Microglia and Promotes Their Phagocytic Activity. Journal of Neuroimmune Pharmacology 6(3): 381-388.

Qin H, Wilson CA, Lee SJ, Zhao X, Benveniste EN (2005). LPS induces CD40 gene expression through the activation of NF- $\{$ kappa $\}$ B and STAT-1 $\{$ alpha $\}$ in macrophages and microglia. Blood 106(9): 3114-3122.

Qin H, Wilson CA, Roberts KL, Baker BJ, Zhao X, Benveniste EN (2006). IL-10 Inhibits Lipopolysaccharide-Induced CD40 Gene Expression through Induction of Suppressor of Cytokine Signaling-3. The Journal of Immunology 177(11): 7761-7771.

Quah BJC, Warren HS, Parish CR (2007). Monitoring lymphocyte proliferation in vitro and in vivo with the intracellular fluorescent dye carboxyfluorescein diacetate succinimidyl ester. Nature Protocols 2(9): 2049-2056. 
Ragonese P, Aridon P, Salemi G, D'Amelio M, Savettieri G (2008). Mortality in multiple sclerosis: a review. European Journal of Neurology 15(2): 123-127.

Rajda C, Bencsik K, VÃ@csei L L, Bergquist J (2002). Catecholamine levels in peripheral blood lymphocytes from multiple sclerosis patients. Journal of Neuroimmunology 124(1-2): 93-100.

Ramanathan RK, Reddy VV, Holbert JM, Belani CP (1996). Pulmonary Infiltrates Following Administration of Paclitaxel. Chest 110(1): 289-292.

Ramtahal J, Jacob A, Das K, Boggild M (2006). Sequential maintenance treatment with glatiramer acetate after mitoxantrone is safe and can limit exposure to immunosuppression in very active, relapsing remitting multiple sclerosis. Journal of Neurology 253(9): 11601164.

Ransohoff RM, Kivisakk P, Kidd G (2003). Three or more routes for leukocyte migration into the central nervous system. Nature Reviews Immunology 3(7): 569-581.

Reyes-García MG, García-Tamayo F (2009). A neurotransmitter system that regulates macrophage pro-inflammatory functions. Journal of Neuroimmunology 216(1-2): 20-31.

Rinner I, Kawashima K, Schauenstein K (1998). Rat lymphocytes produce and secrete acetylcholine in dependence of differentiation and activation. Journal of Neuroimmunology 81(1-2): 31-37.

Riskind PN, Massacesi L, Doolittle TH, Hauser SL (1991). The role of prolactin in autoimmune demyelination: Suppression of experimental allergic encephalomyelitis by bromocriptine. Annals of Neurology 29(5): 542-547.

Rivera E, Gomez H (2011). Chemotherapy resistance in metastatic breast cancer: the evolving role of ixabepilone. Breast Cancer Reseach 12(Suppl 2): S2.

Roberts RL, Nath J, Friedman MM, Gallin JI (1982). Effects of taxol on human neutrophils. The Journal of Immunology 129(5): 2134-2141.

Robinson MJ, Ronchese F, Miller JH, La Flamme AC (2008). Paclitaxel inhibits killing by murine cytotoxic T lymphocytes in vivo but not in vitro. Immunology and Cell Biology 88(3): 291-296.

Rosenzweig HL, Planck SR, Rosenbaum JT (2011). NLRs in immune privileged sites. Current Opinion in Pharmacology 11(4): 423-428.

Roth BL, Sheffler D, Potkin SG (2003). Atypical antipsychotic drug actions: unitary or multiple mechanisms for atypicality'? Clinical Neuroscience Research 3(1-2): 108-117. 
Rourke C, Starr K, Reavill C, Fenwick S, Deadman K, Jones D (2006). Effects of the atypical antipsychotics olanzapine and risperidone on plasma prolactin levels in male rats: a comparison with clinical data. Psychopharmacology 184(1): 107-114.

Ruggieri M, Avolio C, Livrea P, Trojano M (2007). Glatiramer Acetate in Multiple Sclerosis: A Review. CNS Drug Reviews 13(2): 178-191.

Rusan NM, Fagerstrom CJ, Yvon A-MC, Wadsworth P (2001). Cell Cycle-Dependent Changes in Microtubule Dynamics in Living Cells Expressing Green Fluorescent Protein\{alpha\} Tubulin. Molecular Biology of the Cell 12(4): 971-980.

Sabatino JJ, Shires J, Altman JD, Ford ML, Evavold BD (2008). Loss of IFN-y Enables the Expansion of Autoreactive CD4+ T Cells to Induce Experimental Autoimmune Encephalomyelitis by a Nonencephalitogenic Myelin Variant Antigen. The Journal of Immunology 180(7): 4451-4457.

Sadovnick AD, Dyment DA, Ebers GC, Risch NJ, the Canadian Collaborative Study G (1996). Evidence for genetic basis of multiple sclerosis. The Lancet 347(9017): 1728-1730.

Sakaguchi S (2001). Immunologic tolerance maintained by CD25+CD4+ regulatory T cells: their common role in controlling autoimmunity, tumor immunity, and transplantation tolerance. Immunol. Rev. 182: 18-32.

Sakaguchi S, Ono M, Setoguchi R, Yagi H, Hori S, Fehervari Z, et al. (2006).

Foxp3+CD25+CD4+ natural regulatory $\mathrm{T}$ cells in dominant self-tolerance and autoimmune disease. Immunological Reviews 212(1): 8-27.

Sakaguchi S, Wing K, Onishi Y, Prieto-Martin P, Yamaguchi T (2009). Regulatory T cells: how do they suppress immune responses? International Immunology 21(10): 1105-1111.

Sakaguchi S, Yamaguchi T, Nomura T, Ono M (2008). Regulatory T Cells and Immune Tolerance. Cell 133(5): 775-787.

Sako T, Burioka N, Yasuda K, Tomita K, Miyata M, Kurai J, et al. (2004). Cellular Immune Profile in Patients with Non-small Cell Lung Cancer after Weekly Paclitaxel Therapy. Acta Oncologica 43(1): 15-19.

Sanchez-Madrid F, Angel del Pozo M (1999). Leukocyte polarization in cell migration and immune interactions. EMBO Journal 18(3): 501-511.

Sansom DM, Manzotti CN, Zheng Y (2003). What's the difference between CD80 and CD86? Trends in Immunology 24(6): 313-318.

Schall TJ, Bacon K, Toy KJ, Goeddel DV (1990). Selective attraction of monocytes and T lymphocytes of the memory phenotype by cytokine RANTES. Nature 347(6294): 669-671. 
Scheller B, Hehrlein C, Bocksch W, Rutsch W, Haghi D, Dietz U, et al. (2006). Treatment of Coronary In-Stent Restenosis with a Paclitaxel-Coated Balloon Catheter. The New England Journal of Medicine 355(20): 2113-2124.

Schiff PB, Fant J, Horwitz SB (1979). Promotion of microtubule assembly in vitro by taxol. Nature 277(5698): 665-667.

Schotte A, Janssen P, Gommeren W, Luyten W, Gompel P, Lesage A, et al. (1996). Risperidone compared with new and reference antipsychotic drugs: in vitro and in vivo receptor binding. Psychopharmacology 124(1): 57-73.

Schrempf W, Ziemssen T (2007). Glatiramer acetate: Mechanisms of action in multiple sclerosis. Autoimmunity Reviews 6(7): 469-475.

Schroder K, Hertzog PJ, Ravasi T, Hume DA (2004). Interferon-y: an overview of signals, mechanisms and functions. Journal of Leukocyte Biology 75(2): 163-189.

Sedgwick JD, Schwender S, Imrich H, DÃ Irries R, Butcher GW, ter Meulen V (1991). Isolation and direct characterization of resident microglial cells from the normal and inflamed central nervous system. PNAS 88(16): 7438-7442.

Segal AW (2005). How Neutrophils Kill Microbes. Annual review of Immunology 23(1): 197-223.

Segal BM, Dwyer BK, Shevach EM (1998). An Interleukin (IL)-10/IL-12 Immunoregulatory Circuit Controls Susceptibility to Autoimmune Disease. Journal of Experimental Medicine 187(4): 537-546.

Semb KA, Aamdal S, Oian P (1998). Capillary protein leak syndrome appears to explain fluid retention in cancer patients who receive docetaxel treatment. Journal of Clinical Oncology 16(10): 3426-3432.

Sendo T, Itoh Y, Goromaru T, Hirakawa T, Ishida M, Nakano H, et al. (2004). Role of substance $\mathrm{P}$ in hypersensitivity reactions induced by paclitaxel, an anticancer agent. Peptides 25(7): 1205-1208.

Serada S, Fujimoto M, Mihara M, Koike N, Ohsugi Y, Nomura S, et al. (2008). IL-6 blockade inhibits the induction of myelin antigen-specific Th17 cells and Th1 cells in experimental autoimmune encephalomyelitis. PNAS 105(26): 9041-9046.

Shade RJ, Pisters KMW, Huber MH, Fossella F, Perez-Soler R, Shin DM, et al. (1998). Phase I study of paclitaxel administered by ten-day continuous infusion. Investigational New Drugs 16(3): 237-243.

Shamri R, Xenakis JJ, Spencer LA (2011). Eosinophils in innate immunity: An evolving story. Cell and Tissue Research 343(1): 57-83. 
Sharpe AH (2009). Mechanisms of costimulation. Immunological Reviews 229(1): 5-11.

Shaw PJ, Barr MJ, Lukens JR, McGargill MA, Chi H, Mak TW, et al. (2011). Signaling via the RIP2 Adaptor Protein in Central Nervous System-Infiltrating Dendritic Cells Promotes Inflammation and Autoimmunity. Immunity 34(1): 75-84.

Siamon G (2002). Pattern Recognition Receptors: Doubling Up for the Innate Immune Response. Cell 111(7): 927-930.

Simpson S, Blizzard L, Otahal P, Van der Mei I, Taylor B (2011). Latitude is significantly associated with the prevalence of multiple sclerosis: a meta-analysis. Journal of Neurology, Neurosurgery and Psychiatry 82(10): 1132-1141.

Singer B, Ross AP, Tobias K (2011). Oral fingolimod for the treatment of patients with relapsing forms of multiple sclerosis. International Journal of Clinical Practice 65(8): 887-895.

Smith-Garvin JE, Koretzky GA, Jordan MS (2009). T Cell Activation. Annual Review of Immunology 27(1): 591-619.

Soler P, Bernaudin JF, Basset F (1975). Ultrastructure of pulmonary granulomatosis induced in rats by intravenous complete Freund's adjuvant. Virchows Archiv 368(1): 35-50.

Soma D, Kitayama J, Ishigami H, Kaisaki S, Nagawa H (2009). Different Tissue Distribution of Paclitaxel With Intravenous and Intraperitoneal Administration. Journal of Surgical Research 155(1): 142-146.

Song YW, Kim HA, Baek HJ, Lee EB, Chung ES, Hong KM (1998). Paclitaxel reduces anti-dsDNA antibody titer and BUN, prolonging survival in murine lupus. International Journal of Immunopharmacology 20(11): 669-677.

Sorensen PS (2007). The gap between effect of drugs and effectiveness of treatments. Journal of the Neurological Sciences 259(1-2): 128-132.

Sospedra M, Martin R (2005a). Immunology of Multiple sclerosis. Annual Review of Immunology 23: 683-747.

Sospedra M, Martin R (2005b). Immunology of Multiple Sclerosis. Annual Review of Immunology 23(1): 683-747.

Sriram S, Steiner I (2005). Experimental allergic encephalomyelitis: A misleading model of multiple sclerosis. Annals of Neurology 58(6): 939-945.

Starr TK, Jameson SC, Hogquist KA (2003). Positive and negative selection of T cells Annual Review of Immunology 21(1): 139-176. 
Steffen BJ, Butcher EC, Engelhardt B (1994). Evidence for involvement of ICAM-1 and VCAM-1 in lymphocyte interaction with endothelium in experimental autoimmune encephalomyelitis in the central nervous system in the SJL/J mouse. American Journal of Pathology 145(1): 189-201.

Steinman L (2005). Blocking adhesion molecules as therapy for multiple sclerosis: natalizumab. Nature Review Drug Discovery 4(6): 510-518.

Steinman L (2007). A brief history of TH17, the first major revision in the TH1/TH2 hypothesis of T cell-mediated tissue damage. Nature Medicine 13(2): 139-145.

Steinman L (1996). Multiple Sclerosis: A Coordinated Immunological Attack Against Myelin in the Central Nervous System Cell 85: 299-302.

Steinman L (2001). Multiple Sclerosis: A Two-stage Disease Nature Immunology 2(9): 762-764.

Steinman L, Zamvil SS (2005). Virtues and pitfalls of EAE for the development of therapies for multiple sclerosis. Trends in immunology 26(11): 565-571.

Steinman R, Hemmi H, Pulendran B, Ahmed R (2006). Dendritic Cells: Translating Innate to Adaptive Immunity

From Innate Immunity to Immunological Memory. Current Topics in Microbiology and Immunology 311: 17-58.

Stone LAM, Smith MEM, Albert PSP, Bash CNM, Maloni HR, Frank JAM, et al. (1995). Blood-brain barrier disruption on contrast-enhanced MRI in patients with mild relapsingremitting multiple sclerosis: Relationship to course, gender, and age. Neurology 45(6): 1122-1126.

Streilein JW, Stein-Streilein J (2000). Does innate immune privilege exist? Journal of Leukocyte Biology 67(4): 479-487.

Strous RD, Shoenfeld Y (2006). Schizophrenia, autoimmunity and immune system dysregulation: A comprehensive model updated and revisited. Journal of Autoimmunity 27(2): 71-80.

Stuehr DJ, Marletta MA (1985). Mammalian nitrate biosynthesis: mouse macrophages produce nitrite and nitrate in response to Escherichia coli lipopolysaccharide. PNAS 82(22): 7738-7742.

Sugino H, Futamura T, Mitsumoto Y, Maeda K, Marunaka Y (2009). Atypical antipsychotics suppress production of proinflammatory cytokines and up-regulate interleukin-10 in lipopolysaccharide-treated mice. Progress in NeuroPsychopharmacology and Biological Psychiatry 33(2): 303-307. 
Sun J, Zhang X, Broderick M, Fein H (2003). Measurement of Nitric Oxide Production in Biological Systems by Using Griess Reaction Assay. Sensors 3(8): 276-284.

Suttles J, Stout RD (2009). Macrophage CD40 signaling: A pivotal regulator of disease protection and pathogenesis. Seminars in Immunology 21(5): 257-264.

Suzuki E, Ninomiya Y, Umezawa K (2009). Induction of histidine decarboxylase in macrophages inhibited by the novel NF-[kappa]B inhibitor (-)-DHMEQ. Biochemical and Biophysical Research Communications 379(2): 379-383.

Tan CS, Koralnik IJ (2010). Progressive multifocal leukoencephalopathy and other disorders caused by JC virus: clinical features and pathogenesis. The Lancet Neurology 9(4): 425-437.

Tang Q, Bluestone JA (2008). The Foxp3+ regulatory T cell: a jack of all trades, master of regulation. Nature Immunology 9(3): 239-244.

Targoni OS, Baus J, Hofstetter HH, Hesse MD, Karulin AY, Boehm BO, et al. (2001). Frequencies of Neuroantigen-Specific T Cells in the Central Nervous System Versus the Immune Periphery During the Course of Experimental Allergic Encephalomyelitis. Journal of Immunology 166(7): 4757-4764.

Teitelbaum D, Brenner T, Abramsky O, Aharoni R, Sela M, Arnon R (2003). Antibodies to glatiramer acetate do not interfere with its biological functions and therapeutic efficacy. Multiple Sclerosis 9(6): 592-599.

Tepe G, Zeller T, Albrecht T, Heller S, Schwarzw Ãălder U, Beregi J-P, et al. (2008). Local Delivery of Paclitaxel to Inhibit Restenosis during Angioplasty of the Leg. The New England Journal of Medicine 358(7): 689-699.

Terkeltaub RA, Furst DE, Bennett K, Kook KA, Crockett RS, Davis MW (2010). High versus low dosing of oral colchicine for early acute gout flare: Twenty-four-hour outcome of the first multicenter, randomized, double-blind, placebo-controlled, parallel-group, dose-comparison colchicine study. Arthritis \& Rheumatism 62(4): 1060-1068.

Tierney JB, Kharkrang M, La Flamme AC (2009). Type II-activated macrophages suppress the development of experimental autoimmune encephalomyelitis. Immunology and Cell Biology 87(3): 235-240.

Toker A Targeting Antigen Presenting Cells to Treat Autoimmune Inflammation. MSc, Victoria University of Wellington 2009.

Toker A, Slaney CY, Bäckström BT, Harper JL (2011). Glatiramer Acetate Treatment Directly Targets CD11b+Ly6G- Monocytes and Enhances the Suppression of 
Autoreactive T cells in Experimental Autoimmune Encephalomyelitis. Scandinavian Journal of Immunology 74(3): 235-243.

Touil T, Fitzgerald D, Zhang G, Rostami A, Gran B (2006). Cutting Edge: TLR3 Stimulation Suppresses Experimental Autoimmune Encephalomyelitis by Inducing Endogenous IFN-beta. Journal of Immunology 177(11): 7505-7509.

Tran EH, Hoekstra K, Van Rooijen N, Dijkstra CD, Owens T (1998). Immune invasion of the central nervous system parenchyma and experimental allergic encephalomyelitis, but not leukocyte extravasation from blood, are prevented in macrophage-depleted mice. Journal of Immunology 161(7): 3767-3775.

Triggiani M, Petraroli A, Loffredo S, Frattini A, Granata F, Morabito P, et al. (2007). Differentiation of monocytes into macrophages induces the upregulation of histamine $\mathrm{H} 1$ receptor. Journal of Allergy and Clinical Immunology 119(2): 472-481.

Tsai M, Lu Z, Wang J, Yeh T-K, Wientjes M, Au J (2007). Effects of Carrier on Disposition and Antitumor Activity of Intraperitoneal Paclitaxel. Pharmaceutical Research 24(9): 1691-1701.

Tsavaris N, Kosmas C, Vadiaka M, Kanelopoulos P, Boulamatsis D (2002). Immune changes in patients with advanced breast cancer undergoing chemotherapy with taxanes. British Journal of Cancer 87(1): 21-27.

Tuohy VK, Sobel RA, Lees MB (1988). Myelin Proteolipid Protein-Induced Experimental Allergic Encephalomyelitis The Journal of Immunology 140(6): 1868-1873.

Turvey SE, Broide DH (2010). Innate immunity. Journal of Allergy and Clinical Immunology 125(2, Supplement 2): S24-S32.

Vaknin I, Blinder L, Wang L, Gazit R, Shapira E, Genina O, et al. (2008). A common pathway mediated through Toll-like receptors leads to T- and natural killer-cell immunosuppression. Blood 111(3): 1437-1447.

van Os J, Kapur S (2009). Schizophrenia. The Lancet 374(9690): 635-645.

Van Schaick EA, Lechat P, Remmerie BMM, Ko G, Lasseter KC, Mannaert E (2003). Pharmacokinetic comparison of fast-disintegrating and conventional tablet formulations of risperidone in healthy volunteers. Clinical Therapeutics 25(6): 1687-1699.

Vanderlugt CL, Neville KL, Nikcevich KM, Eagar TN, Bluestone JA, Miller SD (2000). Pathologic role and temporal appearance of newly emerging autoepitopes in relapsing experimental autoimmune encephalomyelitis. Journal of Immunology 164(2): 670-678. 
Veldhoen M, Uyttenhove C, van Snick J, Helmby H, Westendorf A, Buer J, et al. (2008). Transforming growth factor-b 'reprograms' the differentiation of T helper 2 cells and promotes an interleukin 9-producing subset. Nature Immunology 9(12): 1341-1346.

Viglietta V, Baecher-Allan C, Weiner HL, Hafler DA (2004). Loss of Functional Suppression by CD4+CD25+ Regulatory T Cells in Patients with Multiple Sclerosis. Journal of Experimental Medicine 199(7): 971-979.

Vignali DAA, Collison LW, Workman CJ (2008). How regulatory T cells work. Nature Reviews Immunology 8(7): 523-532.

Vivier E, Tomasello E, Baratin M, Walzer T, Ugolini S (2008). Functions of natural killer cells. Nature Immunology 9(5): 503-510.

Vollmer T, Panitch H, Bar-Or A, Dunn J, Freedman MS, Gazda SK, et al. (2008). Glatiramer acetate after induction therapy with mitoxantrone in relapsing multiple sclerosis. Multiple Sclerosis 14(5): 663-670.

Voorthuis JAC, Uitdehaag BMJ, De Groot CJA, Goede PH, Van der Meide PH, Dijkstra CD (1990). Suppression of experimental allergic encephalomyelitis by intraventricular administration of interferon-gamma in Lewis rats. Clinical and Experimental Immunology 81(2): 183-188.

Voskoboinik I, Smyth MJ, Trapani JA (2006). Perforin-mediated target-cell death and immune homeostasis. Nature Reviews Immunology 6(12): 940-952.

Vyas JM, Van der Veen AG, Ploegh HL (2008). The known unknowns of antigen processing and presentation. Nature Reviews Immunology 8(8): 607-618.

Waldner H (2009). The role of innate immune responses in autoimmune disease development. Autoimmunity Reviews 8(5): 400-404.

Walker LSK, Abbas AK (2002). The enemy within: keeping self-reactive T cells at bay in the periphery. Nature Reviews Immunology 2(1): 11-19.

Wang GS, Yang KY, Perng RP (2001). Life-threatening hypersensitivity pneumonitis induced by docetaxel (taxotere). British Journal of Cancer 85(9): 1247-1250.

Watanabe T, Noritake J, Kaibuchi K (2005). Regulation of microtubules in cell migration. Trends in Cell Biology 15(2): 76-83.

Weber MS, Prod'homme T, Youssef S, Dunn SE, Rundle CD, Lee L, et al. (2007). Type II monocytes modulate $\mathrm{T}$ cell-mediated central nervous system autoimmune disease. Nature Medicine 13(8): 935-943. 
Weir C, McNeill A, Hook S, Harvie M, La Flamme AC, Le Gros G, et al. (2006). Critical role of preproenkephalin in experimental autoimmune encephalomyelitis. Journal of Neuroimmunology 179: 18-25.

West HL, Crowley JJ, Vance RB, Franklin WA, Livingston RB, Dakhil SR, et al. (2005). Advanced bronchioloalveolar carcinoma: a phase II trial of paclitaxel by 96-hour infusion (SWOG 9714): a Southwest Oncology Group study. Annals of Oncology 16(7): 1076-1080.

Willenborg DO, Fordham S, Bernard CCA, Cowden WB, Ramshaw IA (1996). IFN- $\gamma$ Plays a Critical Down-Regulatory Role in the Induction and Effector Phase of Myelin Oligodendrocyte Glycoprotein-Induced Autoimmune Encephalomyelitis. Journal of Immunology 157(8): 3223-3227.

Willenborg DO, Fordham SA, Staykova MA, Ramshaw IA, Cowden WB (1999). IFN-y Is Critical to the Control of Murine Autoimmune Encephalomyelitis and Regulates Both in the Periphery and in the Target Tissue: A Possible Role for Nitric Oxide. The Journal of Immunology 163(10): 5278-5286.

Willer CJ, Dyment DA, Risch NJ, Sadovnick AD, Ebers GC (2003). Twin concordance and sibling recurrence rates in multiple sclerosis. PNAS 100(22): 12877-12882.

Williams JL, Kithcart AP, Smith KM, Shawler T, Cox GM, Whitacre CC (2011). Memory cells specific for myelin oligodendrocyte glycoprotein (MOG) govern the transfer of experimental autoimmune encephalomyelitis. Journal of Neuroimmunology 234(1-2): 8492.

Wilmes A, Bargh K, Kelly C, Northcote PT, Miller JH (2007). Peloruside A Synergizes with Other Microtubule Stabilizing Agents in Cultured Cancer Cell Lines. Molecular Pharmaceutics 4(2): 269-280.

Wilmes A, O'Sullivan D, Chan A, Chandrahasen C, Paterson I, Northcote PT, et al. (2010a). Synergistic interactions of peloruside A and other microtubule-stabilizing agents and destabilizing agents in cultured human ovarian carcinoma cells and murine T cells. Cancer Chemotherapy and Pharmacology.

Wilmes A, Rawson P, Peng L, McLauchlan D, Northcote P, Jordan T, et al. (2010b). Effects of the microtubule stabilizing agent peloruside A on the proteome of HL-60 cells. Investigational New Drugs 29(4): 544-553.

Wilson EH, Weninger W, Hunter CA (2010). Trafficking of immune cells in the central nervous system. The Journal of Clinical Investigation 120(5): 1368-1379.

Wolinsky JS (2004). Glatiramer acetate for the treatment of multiple sclerosis. Expert Opinion on Pharmacotherapy 5(4): 875-891. 
Xu W, Tabira T (2011). The Role of Natural Killer (NK) Cells in Experimental Autoimmune Encephalomyelitis (EAE) and Multiple Sclerosis (MS). Advances in Neuroimmune Biology 1(1): 87-94.

Yamaguchi I, Hamada K, Yoshida M, Isayama H, Kanazashi S, Takeuchi K (2009). Risperidone Attenuates Local and Systemic Inflammatory Responses to Ameliorate DietInduced Severe Necrotic Pancreatitis in Mice: It May Provide a New Therapy for Acute Pancreatitis. The Journal of Pharmacology and Experimental Theraputics 328(1): 256-262.

Yamazaki T, Akiba H, Koyanagi A, Azuma M, Yagita H, Okumura K (2005). Blockade of B7-H1 on Macrophages Suppresses CD4+ T Cell Proliferation by Augmenting IFN- $\gamma-$ Induced Nitric Oxide Production. The Journal of Immunology 175(3): 1586-1592.

Yousry TA, Major EO, Ryschkewitsch C, Fahle G, Fischer S, Hou J, et al. (2006). Evaluation of Patients Treated with Natalizumab for Progressive Multifocal Leukoencephalopathy. New England Journal of Medicine 354(9): 924-933.

Yu M, Nishiyama A, Trapp BD, Tuohy VK (1996). Interferon-b inhibits progression of relapsing-remitting experimental autoimmune encephalomyelitis. Journal of Neuroimmunology 64(1): 91-100.

Yura M, Takahashi I, Serada M, Koshio T, Nakagami K, Yuki Y, et al. (2001). Role of MOG-stimulated Th1 type [']light up' (GFP+) CD4+T Cells for the Development of Experimental Autoimmune Encephalomyelitis (EAE). Journal of Autoimmunity 17(1): 1725.

Zaccone P, Fehervari Z, Phillips J, Dunne D, Cooke A (2006). Parasitic worms and inflammatory diseases. Parasite Immunology 28(10): 515-523.

Zaffaroni M, Marino F, Bombelli R, Rasini E, Monti M, Ferrari M, et al. (2008). Therapy with interferon-beta modulates endogenous catecholamines in lymphocytes of patients with multiple sclerosis. Experimental Neurology 214(2): 315-321.

Zhai Y, Kronebusch PJ, Simon PM, Borisy GG (1996). Microtubule dynamics at the G2/M transition: abrupt breakdown of cytoplasmic microtubules at nuclear envelope breakdown and implications for spindle morphogenesis. The Journal of Cell Biology 135(1): 201-214.

Zhang J (2007). Yin and yang interplay of IFN-y in inflammation and autoimmune disease. The Journal of Clinical Investigation 117(4): 871-873.

Zhang X, Koldzic DN, Izikson L, Reddy J, Nazareno RF, Sakaguchi S, et al. (2004). IL-10 is involved in the suppression of experimental autoimmune encephalomyelitis by CD25+CD4+ regulatory T cells Vol. 16, pp 249-256.

Zheng LT, Hwang J, Ock J, Lee MG, Lee W-H, Suk K (2008). The antipsychotic spiperone attenuates inflammatory response in cultured microglia via the reduction of 
proinflammatory cytokine expression and nitric oxide production. Journal of Neurochemistry 107(5): 1225-1235.

Zhu J, Yamane H, Cote-Sierra J, Guo L, Paul WE (2006). GATA-3 promotes Th2 responses through three different mechanisms: induction of Th2 cytokine production, selective growth of Th2 cells and inhibition of Th1 cell-specific factors. Cell Research 16(1): 3-10.

Zorro Manrique S, Duque Correa MA, Hoelzinger DB, Dominguez AL, Mirza N, Lin H-H, et al. (2011). Foxp3-positive macrophages display immunosuppressive properties and promote tumor growth. Journal of Experimental Medicine 208(7): 1485-1499. 
Appendices 


\section{Appendix 1}

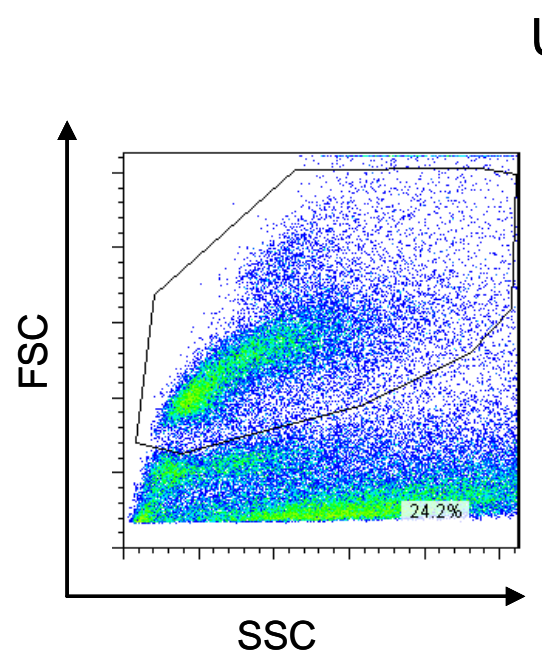

$\mathrm{U} / \mathrm{T}$
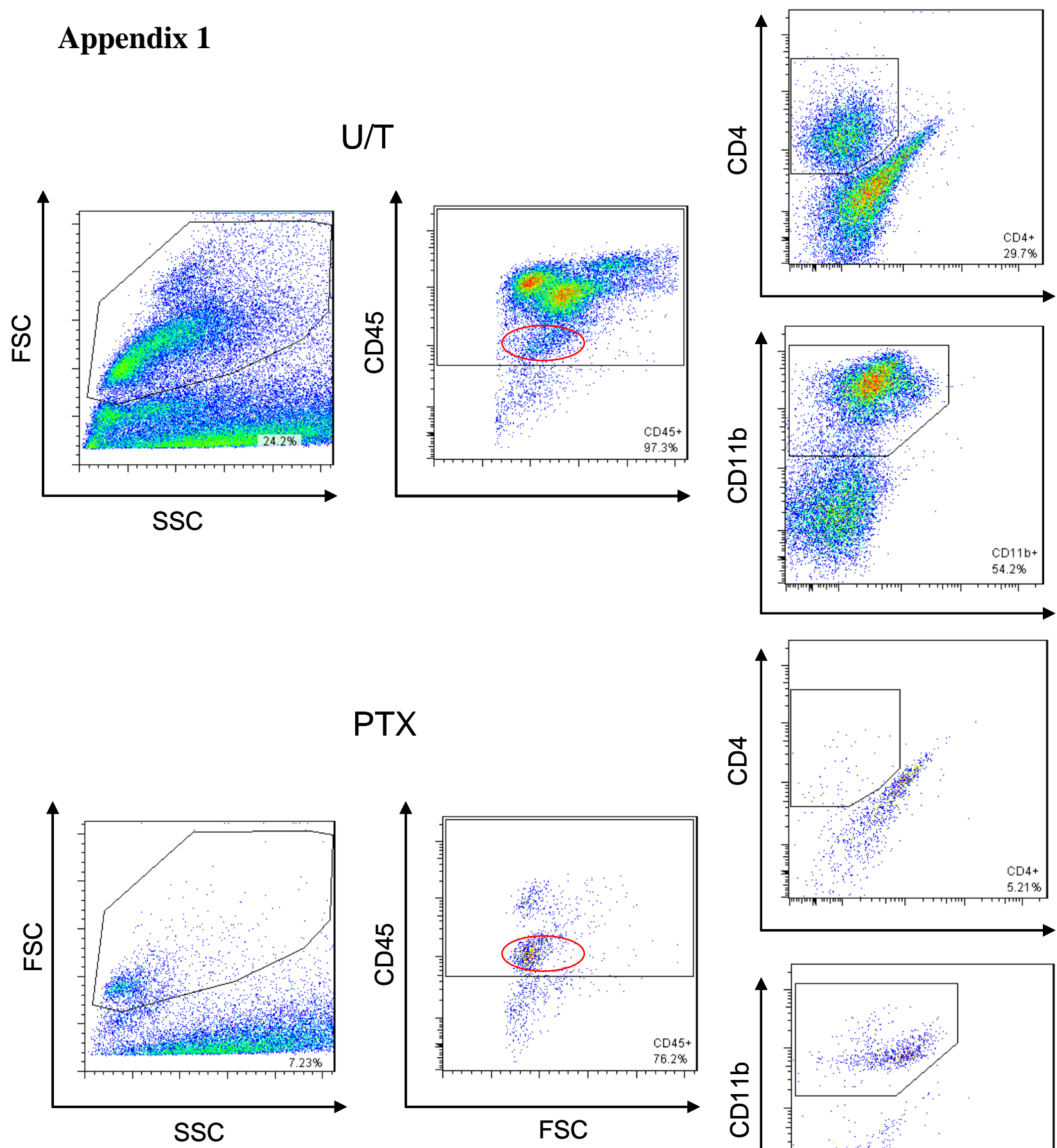

\section{PTX}
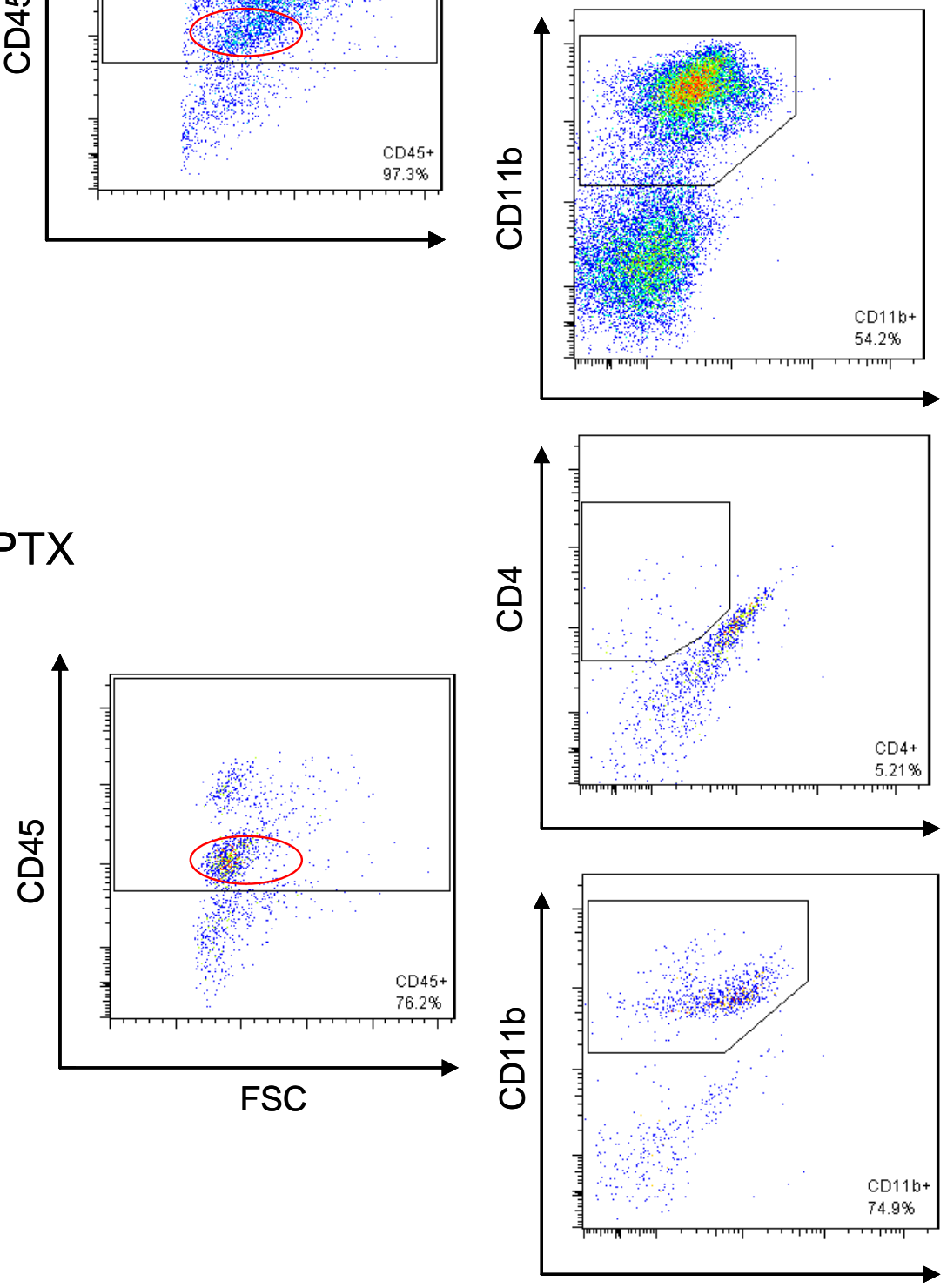

Spinal cord gating scheme Spinal cords were processed as described in the methods and analysed by flow cytometry. Shown are representative plots from one immunised-untreated and one immunised PTX-treated mouse. Microglia (red circles) can be distinguished by their intermediate expression of CD45 relative to high expression in other $\mathrm{CD} 45^{+}$cells (Olson, 2010) . 


\section{Appendix 2}

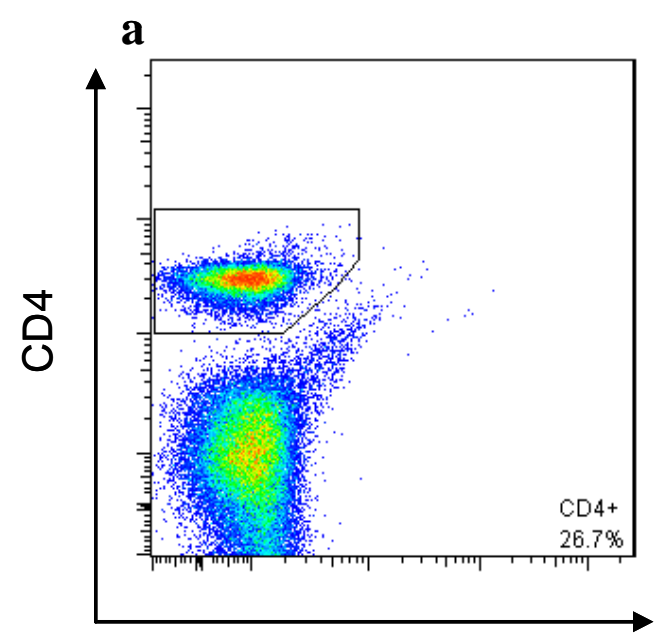

b

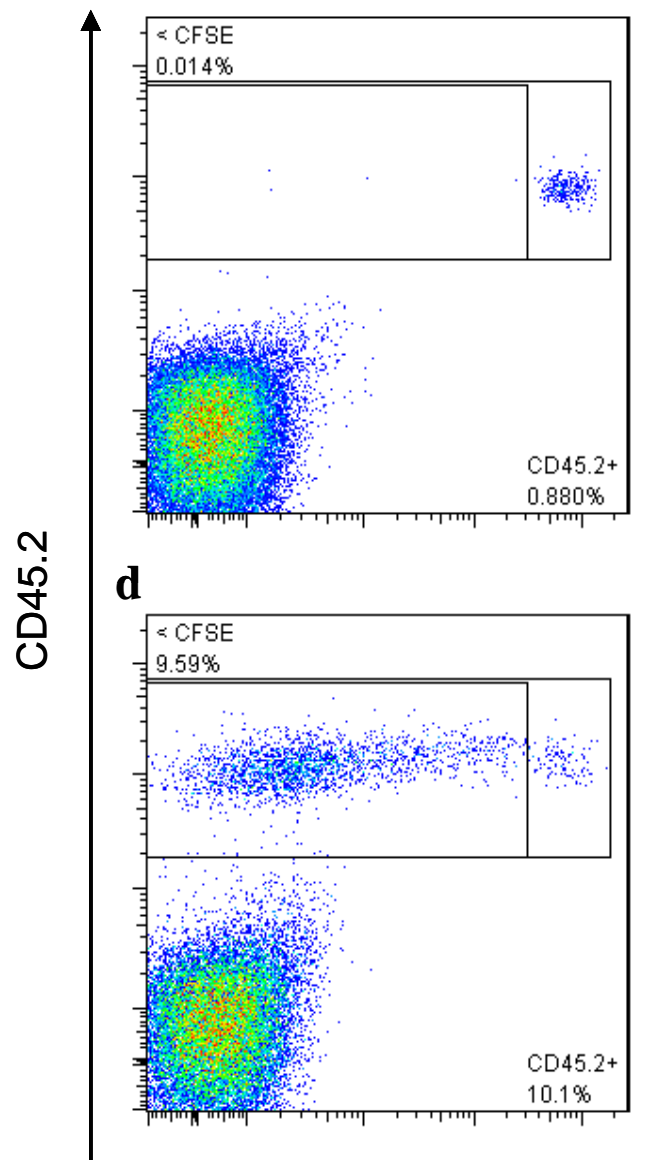

c

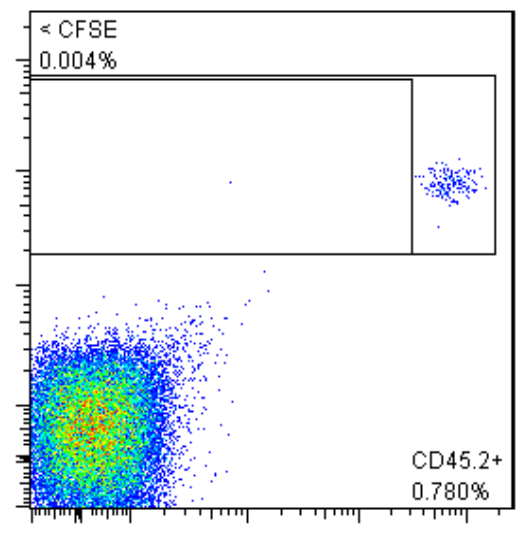

e

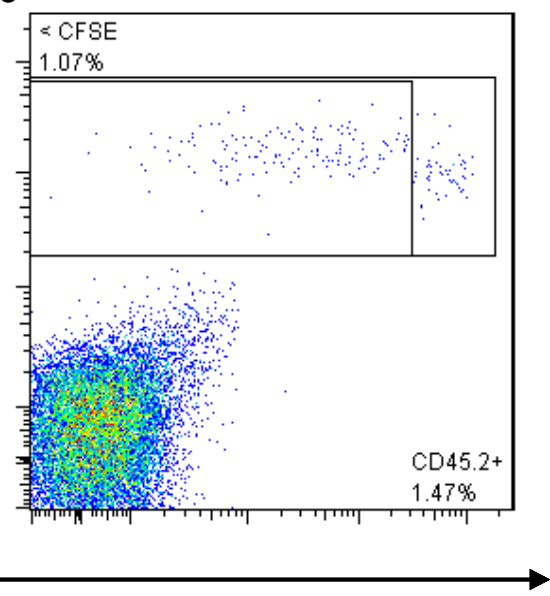

CFSE

Identifying in vivo 2D2 cell proliferation. To identify donor $\mathrm{CD} 4^{+} 2 \mathrm{D} 2$ cells from recipient mice lymphocytes, cells were initially gated on $\mathrm{CD}^{+}$events (a), then on CD45.2 which is expressed on cells from $2 \mathrm{D} 2$ doner mice but not the congenic recipient mice. Total $\mathrm{CD} 4^{+} \mathrm{CD} 45.2^{+}$cells were gated (indicated on plots b,c,d,e as CD45.2 $2^{+}$), a secondary gate (indicated on the plots by $<$CFSE) was placed within the CD45.2 $2^{+}$gate to identify cells that had undergone cell division, as determined by a reduction in CFSE florescence in cell progeny. To identify the percentage CD4=2D2 cells that had undergone proliferation cells events within the <CFSE gate were divided by total events within the CD45.2 $2^{+}$gate. Representative plots of (b) untreated/unimmunised (c) PTX/unimmunised, (d) immunised/untreated (e) PTX/immunised from two experiments ( $n=7 /$ immunised group, $n=4 /$ unimmunised group). 


\section{Appendix 3}

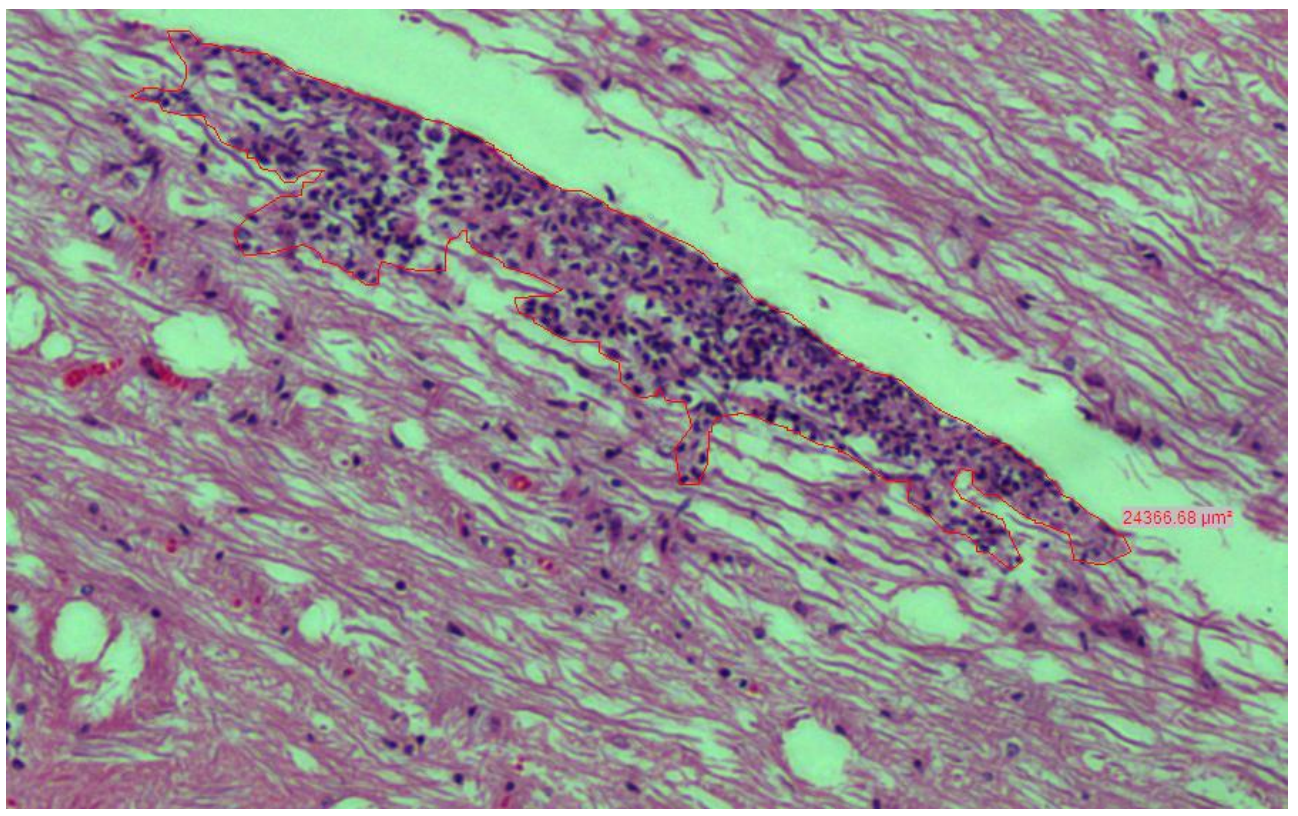

\section{Analysis of lesion area in immunised, risperidone-treated or untreated mice}

$\mathrm{H} \& \mathrm{E}$ stained longitudinal section of spinal cord was analysed for lesions. Analysis was blinded as to if samples were from treated or untreated mice. Images of spinal cord lesions were acquired using an inverted olympus IX51 microscope with cell^A, software, version 3.4 (Olympus, PA, USA). Lesions area was determine by tracing around lesions and calculating total area using with cell^$\wedge^{\wedge}$, software, version 3.4 (Olympus, PA, USA). 


\section{Appendix 4}

\section{Buffers and solutions}

\section{PBS}

$\mathrm{NaCl}$

$145 \mathrm{mM}$

$\mathrm{Na}_{2} \mathrm{HPO}_{4}$

$8.7 \mathrm{mM}$

$\mathrm{NaH}_{2} \mathrm{PO}_{4}$

$1.3 \mathrm{Mm}$

In $\mathrm{ddH}_{2} \mathrm{O}$, autoclaved sterilised.

\section{Pertussis toxin buffer}

Tris

$15 \mathrm{mM}$

Sodium chloride

$0.5 \mathrm{M}$

Triton X-100

$0.017 \%(\mathrm{v} / \mathrm{v})$

In $\mathrm{ddH}_{2} \mathrm{O}$, filter sterilised using $0.22 \mu \mathrm{m}$ syringe filter.

\section{Zinc fixative}

Tris

Calcium acetate

$0.1 \mathrm{M}$

Zinc acetate

$3 \mathrm{mM}$

Zinc chloride

$27 \mathrm{mM}$

$37 \mathrm{mM}$

In $\mathrm{ddH}_{2} \mathrm{O}$, adjusted to $\mathrm{pH}$ 7.4.

Complete $\mathbf{T}$ cell media (CTCM) (v/v)

Dulbecco's Modified Eagle Medium

$85.9 \%$

Fetal calf serum (FCS)

$10 \%$

L-glutamate (200 mM)

$1 \%$

Penicillin/Streptomycin

$1 \%(100 \mathrm{U} / \mathrm{ml} / 10 \mathrm{mg} / \mathrm{ml})$

HEPES buffer (1 M)

$1 \%$

$\beta$-Mecaptoethanol

$0.1 \%$

Non-essential amino acids (10nM)

$0.1 \%$

Wash buffer (v/v)

Dulbecco's Modified Eagle Medium $\quad 96 \%$

HEPES buffer (1 M) 3\%

Penicillin/Streptomycin $\quad 1 \%(100 \mathrm{U} / \mathrm{ml} / 10 \mathrm{mg} / \mathrm{ml})$

FACS buffer (v/v)

FCS

Sodium azide

PBS
$2 \%$

$0.1 \%$

$97.9 \%$ 
Griess solution A (w/v)

Sulphanilamide

$1 \%$

In $2.5 \%$ Phosophoric acid

Griess solution B (w/v)

$\mathrm{N}$-(1-napthyl) ethylenediamine $\quad 1 \%$

In $2.5 \%$ Phosohoric acid

\section{ELISA capture buffer}

$\mathrm{Na}_{2} \mathrm{HPO}_{4}$

$0.1 \mathrm{M}$

In $\mathrm{ddH}_{2} \mathrm{O}$, adjusted to $\mathrm{pH} 9$.

\section{ELISA development stop solution}

$\mathrm{H}_{2} \mathrm{SO}_{4}$

$0.18 \mathrm{M}$

In $\mathrm{ddH}_{2} \mathrm{O}$

\section{Percoll diluent (v)}

10x PBS

$45 \mathrm{mls}$

$3 \mathrm{ml} 0.6 \mathrm{MHCL}$

$3 \mathrm{mls}$

Sterile $\mathrm{ddH}_{2} \mathrm{O}$

$132 \mathrm{mls}$

\section{MTT solution}

MTT $5 \mathrm{mg} / \mathrm{ml}$

(3-(4,5-dimethylthiazol-2-yl)-2.5-diphenyrtetrazolium bromide) In dPBS

Filter sterilised using a $0.22 \mu \mathrm{m}$ syringe driven filter (Biofil, Guangzhou, China)

MTT solubiliser (w/v)

SDS $10 \%$

Dimethylformamide $\quad 45 \%$

In ddH2O, adjusted to $\mathrm{pH} 4.5$ with acetic acid 


\section{Appendix 5}

\begin{tabular}{|c|c|c|c|c|}
\hline Cell Surface Markers & Fluorophore & Clone & Isotype & Manufacturer \\
\hline B220 & $\begin{array}{l}\text { FITC } \\
\text { Biotin }\end{array}$ & $\begin{array}{l}\text { RA3-6B2 } \\
\text { RA3-6B2 }\end{array}$ & $\begin{array}{l}\text { Rat IgG2a } \\
\text { Rat IgG2a }\end{array}$ & $\begin{array}{l}\text { BD Bioscience } \\
\text { BD Bioscience }\end{array}$ \\
\hline $\mathrm{CD} 4$ & $\begin{array}{l}\text { PE } \\
\text { CyC } \\
\text { V500 } \\
\text { PerCP-Cy5.5 } \\
\text { Biotin }\end{array}$ & $\begin{array}{l}\text { RM4-5 } \\
\text { RM4-5 } \\
\text { RM4-5 } \\
\text { RM4-5 } \\
\text { RM4-5 }\end{array}$ & $\begin{array}{l}\text { Rat IgG2a } \\
\text { Rat IgG2a } \\
\text { Rat IgG2a } \\
\text { Rat IgG2a } \\
\text { Rat IgG2a }\end{array}$ & $\begin{array}{l}\text { BD Bioscience } \\
\text { BD Bioscience } \\
\text { BD Bioscience } \\
\text { eBioscience } \\
\text { BD Bioscience }\end{array}$ \\
\hline CD8 & $\begin{array}{l}\text { PE } \\
\text { CyC } \\
\text { PerCP-Cy5.5 } \\
\text { Biotin }\end{array}$ & $\begin{array}{l}53-6.7 \\
53-6.7 \\
53-6.7 \\
53-6.7\end{array}$ & $\begin{array}{l}\text { Rat IgG2a } \\
\text { Rat IgG2a } \\
\text { Rat IgG2a } \\
\text { Rat IgG2a }\end{array}$ & $\begin{array}{l}\text { BD Bioscience } \\
\text { BD Bioscience } \\
\text { eBioscience } \\
\text { BD Bioscience }\end{array}$ \\
\hline CD11b & $\begin{array}{l}\text { FITC } \\
\text { PE } \\
\text { Biotin }\end{array}$ & $\begin{array}{l}\mathrm{M} 1 / 70 \\
\mathrm{M} 1 / 70 \\
\mathrm{M} 1 / 70\end{array}$ & $\begin{array}{l}\text { Rat IgG2b } \\
\text { Rat IgG2b } \\
\text { Rat IgG2b }\end{array}$ & $\begin{array}{l}\text { BD Bioscience } \\
\text { eBioscience } \\
\text { Serotec }\end{array}$ \\
\hline CD16/CD32 (Fc $\gamma R$ RII/III) & Purified & $2.4 \mathrm{G} 2$ & Rat IgG2b & BD Bioscience \\
\hline $\mathrm{CD} 25$ & $\begin{array}{l}\text { FITC } \\
\text { APC }\end{array}$ & $\begin{array}{l}3 \mathrm{C} 7 \\
\text { PC61 }\end{array}$ & $\begin{array}{l}\text { Rat IgG2b } \\
\text { Rat IgG1 }\end{array}$ & $\begin{array}{l}\text { BD Bioscience } \\
\text { BD Bioscience }\end{array}$ \\
\hline CD40 & $\mathrm{PE}$ & $3 / 23$ & Rat IgG2a & BD Bioscience \\
\hline CD44 & $\mathrm{PE}$ & IM7 & Rat IgG2b & eBioscience \\
\hline CD45 & $\begin{array}{l}\text { CyC } \\
\text { V450 } \\
\text { Biotin }\end{array}$ & $\begin{array}{l}30-F 11 \\
30-F 11 \\
30-F 11\end{array}$ & $\begin{array}{l}\text { Rat IgG2b } \\
\text { Rat IgG2b } \\
\text { Rat IgG2b }\end{array}$ & $\begin{array}{l}\text { BD Bioscience } \\
\text { BD Bioscience } \\
\text { eBioscience }\end{array}$ \\
\hline CD62L & $\begin{array}{l}\text { FITC } \\
\text { APC }\end{array}$ & $\begin{array}{l}\text { MEL-14 } \\
\text { MEL-14 }\end{array}$ & $\begin{array}{l}\text { Rat IgG2a } \\
\text { Rat IgG2a }\end{array}$ & $\begin{array}{l}\text { eBioscience } \\
\text { BD Bioscience }\end{array}$ \\
\hline CD45.1 & $\mathrm{PE}$ & $\mathrm{A} 20$ & Rat IgG2a & BD Bioscience \\
\hline CD45.2 & APC & 104 & Rat IgG2a & BD Bioscience \\
\hline CD80 & $\begin{array}{l}\text { FITC } \\
\text { APC }\end{array}$ & $\begin{array}{l}16-10 \mathrm{~A} 1 \\
16-10 \mathrm{~A} 1\end{array}$ & $\begin{array}{l}\text { Hamster IgG2 } \\
\text { Hamster IgG2 }\end{array}$ & $\begin{array}{l}\text { BD Bioscience } \\
\text { BD Bioscience }\end{array}$ \\
\hline $\mathrm{F} 4 / 80$ & $\begin{array}{l}\text { FITC } \\
\text { PerCP-Cy5.5 } \\
\text { Biotin }\end{array}$ & $\begin{array}{l}\text { CI:A3:1 } \\
\text { BM8 } \\
\text { CI:A3:1 }\end{array}$ & $\begin{array}{l}\text { Rat IgG } \\
\text { Rat IgG2a } \\
\text { Rat IgG }\end{array}$ & $\begin{array}{l}\text { Serotec } \\
\text { eBioscience } \\
\text { Serotec }\end{array}$ \\
\hline Gr-1 & $\begin{array}{l}\text { FITC } \\
\text { PE } \\
\text { APC-Cy7 }\end{array}$ & $\begin{array}{l}\text { RB6-8C5 } \\
\text { RB6-8C5 } \\
\text { RB6-8C5 }\end{array}$ & $\begin{array}{l}\text { Rat IgG2b } \\
\text { Rat IgG2b } \\
\text { Rat IgG2b }\end{array}$ & \begin{tabular}{|l} 
eBioscience \\
BD Bioscience \\
BD Bioscience
\end{tabular} \\
\hline $\mathrm{I}-\mathrm{Ab}$ & $\mathrm{PE}$ & AF6-120.1 & Rat IgG2a & BD Bioscience \\
\hline PDL-1 & $\mathrm{PE}$ & MIH5 & Rat IgG2a & eBioscience \\
\hline \multirow[t]{9}{*}{ Isotype control } & FITC & R35-95 & Rat IgG2a & BD Bioscience \\
\hline & $\mathrm{PE}$ & R35-95 & Rat IgG2a & BD Bioscience \\
\hline & $\mathrm{PE}$ & R3-34 & Rat IgG1 & BD Bioscience \\
\hline & $\mathrm{CyC}$ & R35-95 & Rat IgG2a & BD Bioscience \\
\hline & APC & R35-95 & Rat IgG2a & BD Bioscience \\
\hline & APC-Cy7 & A95-1 & Rat IgG2b & BD Bioscience \\
\hline & $\mathrm{V} 450$ & A95-1 & Rat IgG2b & BD Bioscience \\
\hline & V500 & R35-95 & Rat IgG2a & BD Bioscience \\
\hline & Biotin & R35-95 & Rat IgG2a & BD Bioscience \\
\hline
\end{tabular}

